\title{
DIRECT MEASUREMENTS OF TWO PHOTON EXCHANGE ON LEPTON-PROTON ELASTIC SCATTERING USING SIMULTANEOUS ELECTRON-POSITRON BEAMS IN CLAS
}

\author{
by \\ Dasuni Kalhari Adikaram \\ B.S. in physics, September 2006, University of Colombo, Sri Lanka \\ M.S. in Physics, May 2009, Old Dominion University \\ A Dissertation Submitted to the Faculty of \\ Old Dominion University in Partial Fulfillment of the \\ Requirements for the Degree of \\ DOCTOR OF PHILOSOPHY \\ PHYSICS \\ OLD DOMINION UNIVERSITY \\ May 2014
}

Approved by:

Lawrence Weinstein (Director)

Leposava Vuskovic (Member)

Gail E. Dodge (Member)

Anatoly Radyushkin (Member)

Hussein M. Abdel-Wahab (Member) 


\title{
ABSTRACT
}

\section{DIRECT MEASUREMENTS OF TWO PHOTON EXCHANGE ON LEPTON-PROTON ELASTIC SCATTERING USING SIMULTANEOUS ELECTRON-POSITRON BEAMS IN CLAS}

\author{
Dasuni Kalhari Adikaram \\ Old Dominion University, 2014 \\ Director: Dr. Lawrence Weinstein
}

The electric $\left(G_{E}\right)$ and magnetic $\left(G_{M}\right)$ form factors of the proton are fundamental observables which characterize its charge and magnetization distributions. There are two methods to measure the proton form factors: the Rosenbluth separation method and the polarization transfer technique. However, the ratio of the electric and magnetic form factors measured by those methods significantly disagree at momentum transfer $Q^{2}>1 \mathrm{GeV}^{2}$. The most likely explanation of this discrepancy is the inclusion of two-photon exchange (TPE) amplitude contributions to the elastic electron-proton cross section which significantly changes the extraction of $G_{E}$ from the Rosenbluth separation measurement. The Jefferson Lab CLAS TPE experiment determined the TPE contribution by measuring the ratio of positron-proton to electron-proton elastic scattering cross sections. The primary electron beam was used to create an intense bremsstrahlung photon beam. Some of the photons were then converted to a mixed $e^{+} / e^{-}$beam which then interacted with a liquid hydrogen target. The $e^{+} p$ and $e^{-} p$ events were detected by the CLAS (CEBAF Large Acceptance Spectrometer). The elastic cross section ratios $\left(\left(\sigma\left(e^{+} p\right) /\left(\sigma\left(e^{-} p\right)\right)\right.\right.$ were measured over a wide range of virtual photon polarization $\varepsilon$ and $Q^{2}$.

The cross section ratios displayed a strong $\varepsilon$ dependence at $Q^{2}=1.45 \mathrm{GeV}^{2}$. There is no significant $Q^{2}$ dependence observed at $\varepsilon=0.45$. The results are consistent with a recent measurement at the VEPP-3 lepton storage ring in Novosibirsk and with the hadronic calculation by Blunden, Melnitchouk and Tjon. The hadronic calculation resolves the disagreement between the Rosenbluth separation and polarization transfer extractions of $G_{E} / G_{M}$ at $Q^{2}$ up to $2-3 \mathrm{GeV}^{2}$. Applying the CLAS TPE correction to the Rosenbluth cross section measurements significantly decreases the extracted value of $G_{E}$ and brings it into good agreement with the polarization transfer measurement at $Q^{2} \sim 1.75 \mathrm{GeV}^{2}$. Thus, these measurements appear to resolve the proton electric form factor discrepancy for $Q^{2}<2 \mathrm{GeV}^{2}$. 
Copyright, 2014, by Dasuni Kalhari Adikaram, All Rights Reserved. 


\section{ACKNOWLEDGEMENTS}

This work could have not been possible without the help and support of many people. First and foremost, I thank my parents for giving me the greatest gift of all, education and the freedom of choice. I would not be anywhere without them and there is no words that could ever match the love and support you gave since I was born. Amma and Thathta, this work is dedicated to you with love and admiration.

My sincerest appreciations go to my advisor, Dr. Larry Weinstein, for the patient guidance, encouragement and advice he has provided throughout the last six years as his student. I have been extremely fortunate to have a supervisor who cared so much about my work, and who responded to my questions and queries so promptly. I also would like to thank Dr. Brian Raue for his valuable advice and suggestions during the whole analysis process and the encouragement throughout my research. Without their support, I would not complete this thesis and finish the degree.

It is also a great pleasure for me to thank the rest of my thesis committee, Anatoly Radyushkin, Gail Dodge, Leposava Vuskovic and Hussein Abdel-Wahab for their encouragement, guidance, and insightful suggestions throughout my Ph.D. study. I feel proud and honored that you have accepted to be on my committee.

I thank my friend and fellow graduate student, Dipak Rimal, for various discussions during our time as graduate students on the TPE experiment and for his contributions to the analysis in this dissertation. I owe special thanks to Robert Bennett, for always helping me with the computational difficulties and for so many helpful discussions in trying to solve various analysis problems. I would like to thank Dr. John Arrington for the valuable comments and discussions on the analysis and providing the previous measurements and theoretical calculation of positron-proton to electron-proton cross section ratios. I gratefully acknowledge the rest of the TPE group for their various support during the TPE run period and analysis process.

I thank the ODU physics department staff, Lisa, Delicia and Annette, for making everyday life at ODU an easy one.

During my time as a PhD student I have met many nice and inspiring colleagues. This made my bit isolated working life at ODU quite enjoyable. I also express my gratitude to all my Sri Lankan friends in Hampton Roads, who made me home away from home. Thank you all!

I also thank my brother, his wife and my parents-in-law for their understanding 
and support all along these years.

Last, and hence most importantly, I want to thank the two most important men in my life, my wonderful husband and son. I can not find the words to express my appreciation to my husband Duminda who has shown dedication, love and persistent confidence in me. I thank my lovely son, Devin, for his sweet smile and understanding. 


\section{TABLE OF CONTENTS}

LIST OF TABLES ...................... . . . . . . . . . . . . . . .

LIST OF FIGURES . . . . . . . . . . . . . . . . . xiv

Chapter

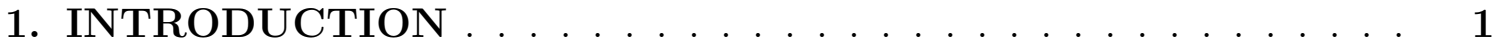

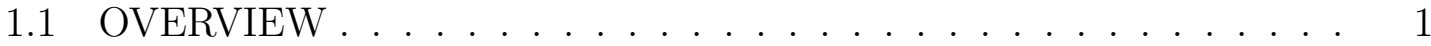

1.2 ELASTIC LEPTON-PROTON SCATTERING . . . . . . . . . . 2

1.3 EXPERIMENTAL MEASUREMENTS OF PROTON FORM FAC-

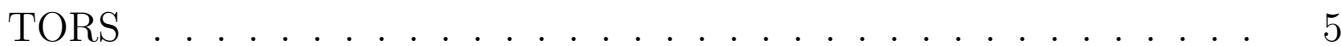

2. TWO PHOTON EXCHANGE . . . . . . . . . . . . . . 13

2.1 PHENOMENOLOGICAL ESTIMATES . . . . . . . . . . . . . . . . . 14

2.2 THEORETICAL CALCULATIONS . . . . . . . . . . . . . . . . . . . . . . . . . . . .

2.3 TPE MEASUREMENTS . . . . . . . . . . . . . . . . . 23

3. TPE EXPERIMENT . . . . . . . . . . . . . . . . . . . . 32

3.1 CEBAF ACCELERATOR . . . . . . . . . . . . 32

3.2 BEAMLINE COMPONENTS . . . . . . . . . . . . . . . . . . 33

3.3 BEAM MONITORS . . . . . . . . . . . . . . . . . . . . 39

3.4 HALL B AND CLAS DETECTOR . . . . . . . . . . . . . . . . . . . . . . . . . . . 43

3.5 DETECTOR SYSTEM . . . . . . . . . . . . . . . . . . . . . . . . 43

3.6 TRIGGER AND DATA ACQUISITION . . . . . . . . . . . . 51

4. DATA ANALYSIS . . . . . . . . . . . . . . . . . . 53

4.1 DATA COLLECTION . . . . . . . . . . . . . . 53

4.2 DETECTOR CALIBRATION AND DATA REDUCTION . . . . . 53

4.3 CUTS AND CORRECTIONS . . . . . . . . . . . . . . 57

5. RESULTS . . . . . . . . . . . . . . . . . . . . . . . . 83

5.1 KINEMATIC COVERAGE AND BIN SELECTION . . . . . . . . . 83

5.2 CHARGE ASYMMETRY RATIO . . . . . . . . . . . . . . . . . . 83

5.3 RESULTS . . . . . . . . . . . . . . . . . 85

5.4 SYSTEMATIC UNCERTAINTY . . . . . . . . . . . . . . . . . . . 87

5.5 RADIATIVE CORRECTIONS . . . . . . . . . . . . . . . . 98

6. DISCUSSION AND CONCLUSION . . . . . . . . . . . . . 102

6.1 DISCUSSION . . . . . . . . . . . . . . . . . . . 102

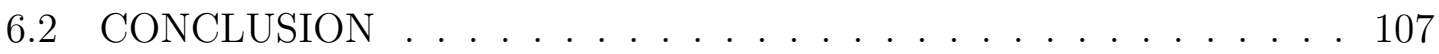


BIBLIOGRAPHY . . . . . . . . . . . . . . . . 109

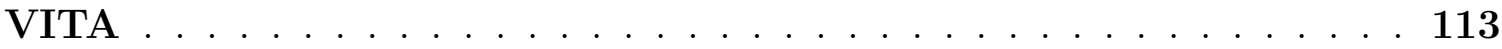




\section{LIST OF TABLES}

Table

Page

1. TPE Run conditions. . . . . . . . . . . . . . . . . . . 39

2. Number of triggers collected in each magnet cycle for each configuration. 54

3. List of bad TOF paddles. . . . . . . . . . . . . . . . . . . . . . . 79

4. Bin ranges and bin centers for $Q^{2}$ and $\varepsilon$ for high $Q^{2}$ data. . . . . . . 86

5. $\quad$ Bin ranges and bin centers for $Q^{2}$ and $\varepsilon$ for low $\varepsilon$ data. . . . . . . . . 88

6. Systematic uncertainties on the quadruple ratio due to various sources at $Q^{2}=1.45 \mathrm{GeV}^{2} \ldots \ldots \ldots \ldots$. . . . . . . . . . . 97

7. Systematic uncertainties on the quadruple ratio due to various sources at $\varepsilon=0.45 . \ldots \ldots \ldots$. . . . . . . . . . . . 97

8. Final $e^{+} p / e^{-} p$ cross section ratios $(\mathrm{R})$ and the associated uncertainties at $Q^{2}=1.45 \mathrm{GeV}^{2} \ldots \ldots \ldots \ldots \ldots$. . . . . . . . . . . 102

9. Final $e^{+} p / e^{-} p$ cross section ratios (R) and the associated uncertainties at $\varepsilon=0.45 \ldots \ldots \ldots$. . . . . . . . . . . . . . 103

10. $\frac{\mu_{p} G_{E}}{G_{M}}$ measurements at $Q^{2}=1.75 \mathrm{GeV}^{2}$ without and with CLAS TPE corrections. . . . . . . . . . . . . . . . 107 


\section{LIST OF FIGURES}

Figure

1. Elastic electron-proton scattering in the one-photon exchange (Born)

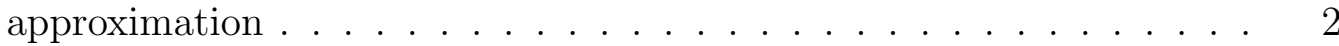

2. Reduced cross section $\sigma_{R}$ as a function of $\varepsilon$ at $Q^{2}=2.5 \mathrm{GeV}^{2}$. . . . 6

3. Normalized $G_{M}$ (top) and $G_{E}$ (bottom) from direct Rosenbluth separation measurements as a function of $Q^{2}$. . . . . . . . . . . . . . . . 7

4. Schematic diagram for the proton recoil polarization in the $\mathrm{H}\left(\vec{e}, e^{\prime} \vec{p}\right)$ reaction. . . . . . . . . . . . . . . . 8

5. The world data on the ratio of electric to magnetic form factors $\frac{\mu_{p} G_{E}}{G_{M}}$ by Rosenbluth separation method and polarization transfer technique.

6. Measurements of $\mu_{p} G_{E} / G_{M}$ from the super Rosenbluth separation method, from previous Rosenbluth separation method and from the polarization transfer technique. . . . . . . . . . . . . . .

7. The reduced cross section measured by Jefferson Lab experiment E01001 and as predicted from polarization transfer measurements at $Q^{2}=$ $2.64 \mathrm{GeV} / \mathrm{c}^{2}$ and $Q^{2}=4.1 \mathrm{GeV} / \mathrm{c}^{2} \ldots \ldots \ldots \ldots$

8. Feynman diagrams of the processes associated with elastic electronproton scattering. $\ldots \ldots \ldots \ldots$

9. Box and crossed-box two-photon exchange contributions to elastic electron-nucleon scattering.

10. The proton form factor ratio measured by the Rosenbluth method and the polarization transfer technique and their polynomial fits. . . . . .

11. The ratio $Y_{2 \gamma}$ as a function of $\varepsilon$ for different $Q^{2}$ from the phenomenological extraction by Guichon and Vanderhaeghen. . . . . . . . . . .

12. The original proton from factor ratios obtained from Rosenbluth method and polarization transfer technique compared with the TPE corrected ratio extracted using phenomenological estimates. . . . . .

13. Difference between the full two-photon exchange correction to the elastic cross section from hadronic calculation and and the commonly used radiative correction from Mo and Tsai. . . . . . . . . . . . . . . . . 
14. The reduced cross section including the effects of adding TPE corrections to the Born cross section. . . . . . . . . . . . . . .

15. The handbag approximation for electron-nucleon elastic scattering. $H$ represents the lepton scatters from the quarks in the nucleon and the lower blob represents the GPD's of the nucleon. . . . . . . . . . . .

16. The ratio of $\mu_{p} G_{E} / G_{M}$ as measured by Rosenbluth separation, as measured by polarization transfer technique and as measured by Rosenbluth and corrected for TPE effects in the partonic model. . . . . . . 21

17. Electron-proton elastic scattering with hard two photon exchange. . . 22

18. $\frac{\sigma_{R}}{\left(\mu_{p} G_{D}\right)^{2}}$ as a function of $\varepsilon$ at $Q^{2}=3.25$ and $4.0 \mathrm{GeV}^{2} \ldots \ldots . . . .23$

19. The ratio of positron-proton to electron- proton elastic cross section, $R$ as a function of $Q^{2}$. . . . . . . . . . . . . . . . . 28

20. The ratio of positron-proton to electron- proton elastic cross section, $R$ as a function of $\varepsilon$ for the measurements below $Q^{2}=2 \mathrm{GeV}^{2}$. . . .

21. Comparison of the preliminary results for the Ratio $R=\frac{\sigma\left(e^{+} p\right)}{\sigma\left(e^{-} p\right)}$ from the Novosibirsk experiment with previous world data and theoretical calculations. . . . . . . . . . . . . . . . . . . . 30

22. A comparison of CLAS TPE test run results at $Q^{2} \approx 0.206 \mathrm{GeV}^{2}$ with previous world data at $Q^{2}<2 \mathrm{GeV}^{2}$. . . . . . . . . . . . . 31

23. Beamline sketch for the CLAS TPE experiment . . . . . . . . . . 33

24. A schematic layout of the CEBAF accelerator. . . . . . . . . . . . 34

25. The simulated trajectories of the leptons through the chicane magnetic system. . . . . . . . . . . . . . . . . 36

26. An engineering drawing and a photograph of the TPE liquid hydrogen

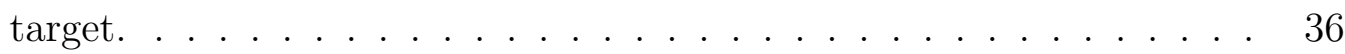

27. Photographs of the shielding taken during engineering work. . . . . 38

28. The TPE sparse fiber monitor. . . . . . . . . . . . . . . . . . . . 40

29. The $x$-horizontal and $y$-vertical beam position distributions measured by the sparse fiber monitor. . . . . . . . . . . . . . . . . . . 41 
30. Individual positron and electron beam position centroids as a function of Italian Dipole current and magnetic field as measured by the sparse fiber monitor. . . . . . . . . . . . . . . . . . . . . . . 4 41

31. The hit distribution for the left beam passing through the chicane as measured by the dense fiber monitor. . . . . . . . . . . . . . . . . . . 42

32. A photograph of a TPE calorimeter taken during the assembly process. 43

33. A schematic layout of the CLAS detector cut along the beamline and cut perpendicular to beam. . . . . . . . . . . . . . . . . 44

34. Contours of constant absolute magnetic field for the CLAS toroid in the midplane between two coils. . . . . . . . . . . . . . . . . . . . 46

35. GEANT4 simulation of the target area with the DC R1 mother volume, target and mass shielding. . . . . . . . . . . . . . . . . . 47

36. Horizontal cut through the CLAS detector at beam line elevation and the schematic view of a typical drift-chamber sector. . . . . . . . . . . 48

37. A portion of the two super layers of R3. . . . . . . . . . . . . . 48

38. The four panels of TOF scintillator counters for one of the sectors. . . 49

39. One sector of the CLAS electromagnetic calorimeter. . . . . . . . . 50

40. The offset corrected $\Delta t$ as a function proton momentum. . . . . . . . 56

41. Mean of the DC residual distribution vs. run number. . . . . . . . . 58

42. Width (standard deviation) of a double gaussian fit to the DC residual distribution vs. run number. . . . . . . . . . . . . . . . . 58

43. $\Delta E_{\text {Incident }}$ vs. $\Delta E_{e}$ distributions for $e^{-} p$ events and $e^{+} p$ events. . . . 60

44. $\Delta \phi\left(^{\circ}\right)$ distributions for different $Q^{2}$ and $\varepsilon$ bins. . . . . . . . . . . . 61

45. $\Delta p_{p}(\mathrm{GeV})$ distributions for different $Q^{2}$ and $\varepsilon$ bins. . . . . . . . . 61

46. $\Delta E^{+}(\mathrm{GeV})$ distributions for different $Q^{2}$ and $\varepsilon$ bins. . . . . . . . 62

47. $\Delta E^{-}(\mathrm{GeV})$ distributions for different $Q^{2}$ and $\varepsilon$ bins. . . . . . . . . 62

48. $\Delta E^{+}$distributions for different $Q^{2}$ bins for $0.1<\varepsilon<0.5$ with positive torus magnet setting. . . . . . . . . . . . . . . 63 
49. $\Delta E^{+}$distributions for different $Q^{2}$ bins for $0.1<\varepsilon<0.5$ with negative torus magnet setting. . . . . . . . . . . . . . . . . . . . . . . 64

50. $Q^{2}$ dependence of the means and the widths of the Gaussian functions fit to $\Delta \phi$ distributions for different $\varepsilon$ ranges. . . . . . . . . . . . . . . 64

51. $Q^{2}$ dependence of the means and the widths of the Gaussian functions fit to $\Delta p_{p}$ distributions for different $\varepsilon$ ranges. . . . . . . . . . . . . 65

52. $Q^{2}$ dependence of the means and the widths of the Gaussian functions fit to $\Delta E^{+}$distributions for different $\varepsilon$ ranges. . . . . . . . . . . . 65

53. $Q^{2}$ dependence of the means and the widths of the Gaussian functions fit to $\Delta E^{-}$distributions for different $\varepsilon$ ranges. . . . . . . . . . . . . . 66

54. The four variables, $\Delta \phi, \Delta p_{p}$ and $\Delta E^{ \pm}$, before and after applying the other three elastic cuts on each. . . . . . . . . . . . . . 66

55. nvariant mass distribution of the $\mathrm{H}\left(e, e^{\prime}\right)$ elastic peak for $2.2 \mathrm{GeV}$ incident electrons before momentum correction. . . . . . . . . . . . 67

56. Before momentum corrections: relative difference between the measured and calculated electron momentum $(\Delta p / p)$ vs. azimuthal angle $\phi$ for elastic scattering for all six sectors of CLAS. . . . . . . . . . .

57. After momentum corrections: relative difference between measured and calculated electron momentum $(\Delta p / p)$ vs. azimuthal angle $\phi$ for $2.2 \mathrm{GeV}$ elastic scattering events. . . . . . . . . . . . . .

58. $\Delta t$ distribution (uncorrected) as a function of proton momentum, $p_{p}$ for each magnet cycle. . . . . . . . . . . . . . . . . .

59. Paddle by paddle $\Delta t$ distribution of sector 2 in magnet cycle 2,3 and 4. Four scintillator paddles $(26,44,45,49)$ needed to be recalibrated.

60. Offset-corrected $\Delta t$ distribution as a function of proton momentum. . 73

61. Lepton vertex distribution $\left(v z_{l}\right) \ldots \ldots \ldots \ldots$. . . . . . . . 74

62. The difference between the lepton vertex $\left(v z_{l}\right)$ and the proton vertex $\left(v z_{p}\right) \ldots \ldots \ldots \ldots \ldots \ldots \ldots \ldots$

63. Reconstructed incident energy distributions for elastic scattering events using scattering angles $\left(E_{\text {Incident }}^{1}\right)$ for positive and negative chicane magnet settings. . . . . . . . . . . . . . . . . . . 
64. Reconstructed incident beam energy distributions of elastic scattering events using scattering angles $\left(E_{\text {Incident }}^{1}\right) \ldots \ldots \ldots 76$

65. The number of reconstructed electrons as a function of $\theta$ and $\phi$ for positive torus magnet setting (in-bending tracks) for all sectors for different momentum bins.

66. The number of reconstructed electrons as a function of $\theta$ and $\phi$ for negative torus magnet setting (out-bending tracks) for all sectors for different momentum bins.

67. $\theta$-shift vs. momentum of reconstructed electrons for positive torus magnet setting. . . . . . . . . . . . . . . . . . . 78

68. $\Delta \phi$ distributions for different $Q^{2}$ bins for $0.1<\varepsilon<0.5$ after other elastic cuts. . . . . . . . . . . . . . . . . . 80

69. $\Delta E^{-}$and $\Delta \phi$ distributions at $\varepsilon=(0.2,0.5)$ and $Q^{2}=(1.0,4.0) . \quad . \quad 81$

70. $\Delta \phi$ distributions with Gaussian fits to the backgrounds for $e^{-} p$ and $e^{+} p$ events for data at $\varepsilon=(0.2,0.5)$ and $Q^{2}=(1.0,4.0) \ldots \ldots$. . .

71. The $Q^{2}$ vs. $\varepsilon$ distributions for the $e^{-} p$ and $e^{+} p$ elastic scattering events for both torus magnetic settings. . . . . . . . . . . . . . . . . . 84

72. The bin boundaries for high $Q^{2}$ data and low $\varepsilon$ data. . . . . . . . . . 84

73. Double ratios for positive and negative chicane polarities and the quadruple ratio as a function $\varepsilon$ at $Q^{2} \sim 1.45 \mathrm{GeV}^{2}$. . . . . . . . . 87

74. Double ratios for positive and negative chicane polarities and the quadruple ratio as a function of $Q^{2}$ at $\varepsilon \sim 0.45$. . . . . . . . . . 88

75. The ratio of the incident positron energy density to the incident electron energy density as a function of incident energy before and after the Jan 23 chicane flip. . . . . . . . . . . . . . . . . . .

76. The double ratios for different magnet cycles at $\varepsilon=0.88$ for (top) positive and (bottom) negative chicane magnet settings . . . . . . . . 91

77. The ratio of the incident positron energy density to the incident electron energy density as a function of incident energy before and after the Jan 14 chicane flip. . . . . . . . . . . . . . . . . . . . 92

78. The quadruple ratios for different sectors at $Q^{2}=1.45 \mathrm{GeV}^{2} \ldots$. . . 93

79. The quadruple ratios for different sectors at $\varepsilon=0.45$. . . . . . . . . 94 
80. The quadruple ratios at $Q^{2}=1.45 \mathrm{GeV}^{2}$ background subtracted with different fitting ranges. . . . . . . . . . . . . . . . . 94

81. The quadruple ratios at $\varepsilon=0.45$ background subtracted with different fitting ranges. . . . . . . . . . . . . . . . . . 95

82. The quadruple ratios at $Q^{2}=1.45 \mathrm{GeV}^{2}$ with $3 \sigma$ and $3.5 \sigma$ kinematic cuts. . . . . . . . . . . . . . . . 96

83. Background to signal ratios at $Q^{2}=1.45 \mathrm{GeV}^{2}$ with $3 \sigma$ and $3.5 \sigma$ kinematic cuts. . . . . . . . . . . . . . . . 98

84. The quadruple ratios at $\varepsilon=0.45$ with $3 \sigma$ and $3.5 \sigma$ kinematic cuts. . $\quad 99$

85. The quadruple ratios at $Q^{2}=1.45 \mathrm{GeV}^{2}$ with nominal and tighter fiducial cuts. . . . . . . . . . . . . . . . . 99

86. The quadruple ratios at $\varepsilon=0.45$ with nominal and tighter fiducial cuts. 100

87. The quadruple ratios at $Q^{2}=1.45 \mathrm{GeV}^{2}$ with and without radiative corrections. . . . . . . . . . . . . . . 100

88. The quadruple ratios at $\varepsilon=0.45$ with and without radiative corrections. 101

89. The comparison of CLAS TPE measurements at $Q^{2}=1.45 \mathrm{GeV}^{2}$ with previous world measurements and a theoretical calculation. . . . . . . 104

90. The comparison of CLAS TPE measurements at $\varepsilon=0.45$ with previous world measurements. . . . . . . . . . . . . . . . . . . . . 105

91. The quadruple ratio as a function of $\varepsilon$ at $Q^{2} \sim 1.45 \mathrm{GeV}^{2}$ with a linear fit. . . . . . . . . . . . . . . . . . 105

92. Reduced cross section normalized by the square of the dipole form factor $\left(G_{D}^{2}\right)$ as a function of $\varepsilon$ at $Q^{2} \sim 1.75 \mathrm{GeV}^{2}$. . . . . . . . 106

93. The proton form factor ratio, $\frac{\mu_{p} G_{E}}{G_{M}}$, measured at $Q^{2} \sim 1.75 \mathrm{GeV}^{2}$. . . 107

94. TPE corrected reduced cross sections as a function of $\varepsilon$ at $Q^{2} \sim 1.75$ $\mathrm{GeV}^{2}$ with additional cases. . . . . . . . . . . . . . 108

95. Comparison of polarization measurements and Rosenbluth separation measurements with no TPE corrections and with TPE corrections. . . 109 


\section{CHAPTER 1}

\section{INTRODUCTION}

\subsection{OVERVIEW}

The electric and magnetic form factors characterize the distributions of charge and magnetization in the nucleon. Therefore it is very important to have full quantitative knowledge of these form factors in the study of hadronic physics. Because of their point like structure, electrons and muons are ideal probes of the structure of hadrons via electromagnetic interactions. The Rosenbluth separation method was the first and standard method of extracting the proton form factors from elastic electron-proton scattering for many years. Then there were new measurements of proton form factors obtained from polarization transfer experiments carried out at Jefferson Lab. The polarization transfer measurements were notably precise, but their results significantly disagree with those obtained from Rosenbluth separations. This discrepancy has been known for more than a decade. Several theoretical and experimental investigations have been conducted to understand the possible sources and resolve the discrepancy. These studies suggest that the most likely explanation for the discrepancy of the proton form factor measurements is a two photon exchange (TPE) correction that needs to be included in measurements as a part of radiative corrections.

There have been several theoretical approaches to calculate the TPE contribution and those calculations indicates that the TPE corrections can resolve most of the observed form factor discrepancy. But it is always important to have experimental verifications of these calculations. The TPE correction is directly proportional to the ratio of positron-proton to electron-proton elastic scattering cross sections. The current world data on this cross section ratio is not precise enough to make a strong conclusion of the TPE effect. Three experiments are currently in progress to fulfill this task. The first experiment was carried out at the VEPP-3 electron/positron storage ring in Novosibirsk using an internal hydrogen gas target. Some results have been published and others will be published soon. The CLAS TPE experiment was 


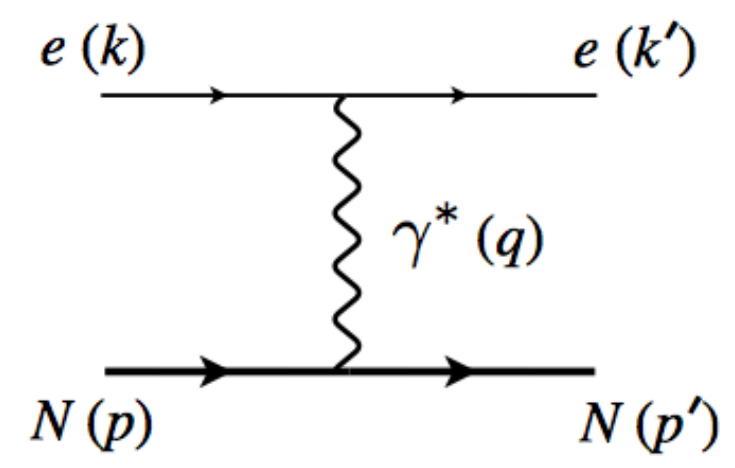

FIG. 1. Elastic electron-proton scattering in the one-photon exchange (Born) approximation. Particle momenta are indicated in parentheses.

performed in Jefferson Lab Hall B using a simultaneous external electron-positron beam. Finally, the OLYMPUS experiment collected the data at the DORIS lepton storage ring at DESY also using an internal gas target. All three experiments have completed data collection and now are in different stages of data analysis. The results from these experiments cover a large kinematic range and will test current theoretical calculations of the TPE correction.

In this work we will present an extensive description of the CLAS TPE experiment. In this chapter we will discuss proton form factor measurements and two photon exchange corrections in detail. The TPE experiment, data analysis and the results will be discussed in later chapters.

\subsection{ELASTIC LEPTON-PROTON SCATTERING}

The general formalism of elastic electron-proton scattering in the one-photo exchange (Born) approximation is discussed in this section.

\subsubsection{KINEMATICS}

Figure 1 shows the Feynman diagram for elastic electron-proton (or positronproton) scattering in the one-photon exchange (Born) approximation. Here $k$ and $k^{\prime}$ are the initial and final four-momenta of the electron, with corresponding energies $E$ and $E^{\prime}$. The initial and final four momenta of the proton are given by $p$ and $p^{\prime}$, respectively. The four-momentum transfer from the lepton to the nucleon is given 
by

$$
q=k-k^{\prime}=p^{\prime}-p \text { and } Q^{2}=4 E E^{\prime} \sin ^{2}(\theta / 2) \equiv-q^{2}(>0)
$$

The scattering cross section is generally defined in terms of $Q^{2}$, scattering angle $\theta$ and the dimensionless quantities, $\tau$ and $\varepsilon$.

$$
\begin{aligned}
\tau & =\frac{Q^{2}}{4 M^{2}} \\
\varepsilon & =\left(1+2(1+\tau) \tan ^{2} \frac{\theta}{2}\right)^{-1}
\end{aligned}
$$

Where $M$ is the mass of the proton and $\varepsilon$ is the relative flux of longitudinal virtual photons.

In the Born approximation, the positron-proton(+)/electron-proton(-) scattering invariant amplitude can be written as

$$
\mathcal{M}_{\gamma}^{ \pm}= \pm \frac{e^{2}}{q^{2}} j_{\gamma \mu} J_{\gamma}^{\mu}
$$

where $e$ is the electron charge. $j_{\gamma \mu}$ and $J_{\gamma}^{\mu}$ are the electron and proton currents, respectively and can be formulated in terms of the lepton $\left(u_{e}\right)$ and proton $\left(u_{N}\right)$ spinors as follows.

$$
\begin{aligned}
j_{\gamma \mu} & =\bar{u}_{e}\left(k^{\prime}\right) \gamma_{\mu} u_{e}(k) \\
J_{\gamma}^{\mu} & =\bar{u}_{N}\left(p^{\prime}\right) \Gamma_{\gamma}^{\mu}(q) u_{N}(p)
\end{aligned}
$$

The electromagnetic hadron current operator $\Gamma_{\gamma}^{\mu}$ is parametrized by the Dirac $\left(F_{1}\right)$ and Pauli $\left(F_{2}\right)$ form factors as

$$
\Gamma_{\gamma}^{\mu}(q)=\gamma^{\mu} F_{1}\left(Q^{2}\right)+\frac{i \sigma^{\mu \nu} q_{\nu}}{2 M} F_{2}\left(Q^{2}\right)
$$

The differential cross section of the electron-proton scattering can then be written in terms of proton form factors as follows:

$$
\frac{d \sigma}{d \Omega}=\frac{\left|M_{\gamma}\right|^{2}}{4\left((k \cdot p)^{2}-m_{e}^{2} M^{2}\right)}(2 \pi)^{4} \delta^{4}\left(k^{\prime}+p-k-p^{\prime}\right) \frac{d^{3} \vec{k}^{\prime} d^{3} \vec{p}^{\prime}}{(2 \pi)^{3} 2 E^{\prime}(2 \pi)^{3} 2(M+\omega)}
$$

Here $m_{e}$ is the mass of the lepton and $\omega=E-E^{\prime}$ is the energy of the virtual photon. $\left|M_{\gamma}\right|^{2}$ in Eq. (8) can be expressed in terms of the electron and proton currents. Once the $\delta$ function integrates, the differential cross section can be expressed in terms of Dirac and Pauli form factors as follows: 


$$
\begin{aligned}
\frac{d \sigma}{d \Omega d E^{\prime}}= & \frac{\alpha^{2}}{4 E^{2} \sin ^{4}\left(\frac{\theta}{2}\right)} \frac{E^{\prime}}{E} \delta\left(E-E^{\prime}-\frac{Q^{2}}{2 M}\right) \\
& \left\{\left(F_{1}^{2}+\frac{\kappa^{2} Q^{2}}{4 M^{2}} F_{2}^{2}\right) \cos ^{2}\left(\frac{\theta}{2}\right)-\frac{q^{2}}{2 M^{2}}\left(F_{1}+\kappa F_{2}\right) \sin ^{2}\left(\frac{\theta}{2}\right)\right\}
\end{aligned}
$$

At a given energy $E$ and angle $\theta$, the elastic differential cross section is a $\delta$-function in $E^{\prime}$ at $E^{\prime}=E-\frac{Q^{2}}{2 M}$. If we integrate Eq. (9) over $E^{\prime}$ and divide the numerator and denominator by $\cos ^{2}\left(\frac{\theta}{2}\right)$, the elastic lepton-proton scattering cross section can be written as:

$$
\frac{d \sigma}{d \Omega}=\sigma_{\mathrm{mott}}\left\{\left(F_{1}^{2}+\frac{\kappa^{2} Q^{2}}{4 M^{2}} F_{2}^{2}\right)-\frac{q^{2}}{2 M^{2}}\left(F_{1}+\kappa F_{2}\right) \tan ^{2} \frac{\theta}{2}\right\}
$$

where the mott cross section, $\sigma_{\text {mott }}$, is the scattering cross section for an ultrarelativistic electron from a point-like particle:

$$
\sigma_{\text {mott }}=\frac{\alpha^{2} \cos ^{2}(\theta / 2)}{4 E^{2} \sin ^{4}(\theta / 2)} \frac{E^{\prime}}{E}
$$

Here $\alpha \approx 1 / 137$ is the fine structure constant and the term $\frac{E^{\prime}}{E}$ is due to the recoil of the proton. Although the protons are massive, their recoil effect can not be neglected at high momentum transfer. The Dirac and Pauli form factors can be converted into Sachs electric and magnetic form factors as follows.

$$
\begin{aligned}
G_{E}\left(Q^{2}\right) & =F_{1}-\frac{\kappa Q^{2}}{4 M^{2}} F_{2}=F_{1}-\kappa \tau F_{2} \\
G_{M}\left(Q^{2}\right) & =F_{1}+\kappa F_{2}
\end{aligned}
$$

Then, the scattering cross section can be re-written as

$$
\frac{d \sigma}{d \Omega}=\frac{\sigma_{\mathrm{mott}}}{\varepsilon(1+\tau)}\left\{\varepsilon G_{E}^{2}\left(Q^{2}\right)+\tau G_{M}^{2}\left(Q^{2}\right)\right\}
$$

The reduced Born cross section is given by

$$
\sigma_{R}=\varepsilon G_{E}^{2}\left(Q^{2}\right)+\tau G_{M}^{2}\left(Q^{2}\right)
$$

In the limit as $Q^{2} \rightarrow 0$, which corresponds to the exchange of a low virtuality photon, the electric and magnetic form factors become

$$
G_{E}(0)=1 \text { and } G_{M}(0)=\mu_{p}
$$

where $\mu_{p}=1+\kappa$ is the proton magnetic moment. 
The physical meaning of the electric and magnetic form factors, $G_{E}$ and $G_{M}$, is best understood when the hadronic current is written in the Breit frame. In the Breit frame the scattered electron transfers momentum $\vec{q}$ but no energy $(\omega=0)$ and only in this frame and in the non-relativistic limit $\left(Q^{2} \ll M^{2}\right), G_{E}$ and $G_{M}$ can be interpreted as the Fourier transformations of the spatial distributions of the charge density $\rho_{c h}(\vec{r})$ and the magnetic moment density $\rho_{\text {mag }}(\vec{r})$ :

$$
\begin{gathered}
G_{E}\left(Q^{2}\right)=\int \rho_{c h}(r) e^{-i \vec{q} \cdot \vec{r}} d^{3} r \\
G_{M}\left(Q^{2}\right)=\mu_{p} \int \rho_{\text {mag }}(r) e^{-i \vec{q} \cdot \vec{r}} d^{3} r
\end{gathered}
$$

If $G_{E}$ and $G_{M}$ are known, these physical interpretations immediately allow a calculation of the r.m.s radius of the charge and magnetic moment distribution inside the proton.

$$
\left\langle r_{c h}{ }^{2}\right\rangle=-\left.6 \frac{d G_{E}\left(Q^{2}\right)}{d Q^{2}}\right|_{Q^{2}=0} \quad\left\langle r_{m a g}{ }^{2}\right\rangle=-\left.\frac{6}{\mu_{p}} \frac{d G_{M}\left(Q^{2}\right)}{d Q^{2}}\right|_{Q^{2}=0}
$$

In the relativistic limit where $Q^{2} \gg M^{2}$, one needs to take the effect of relativity into account. The relativistic relationship between Sachs form factors and the proton charge and magnetization distributions was examined by Kelly [1] and it accounts for the Lorentz contraction of the densities in the Breit frame relative to the rest frame.

\subsection{EXPERIMENTAL MEASUREMENTS OF PROTON FORM FACTORS}

Measurement of the proton form factors, $G_{E}$ and $G_{M}$, are very important in order to understand the structure of the proton. The following section discusses the experimental method used to measure those form factors.

\subsubsection{ROSENBLUTH SEPARATION METHOD}

The first technique used to extract the proton form factors was the Rosenbluth separation or the longitudinal-transverse (LT) separation method [2]. According to Eq. (15), $G_{E}^{2}$ and $G_{M}^{2}$ can be extracted by measuring $\sigma_{R}$ at fixed $Q^{2}$ for different values of $\varepsilon$. This can be done by measuring $\sigma_{R}$ at different lepton scattering angles while varying the incident lepton energy to keep $Q^{2}$ fixed. See Fig. 2. This method gives $G_{E}^{2}$ as the slope and $G_{M}^{2}$ as the intercept. At larger scattering angles where 


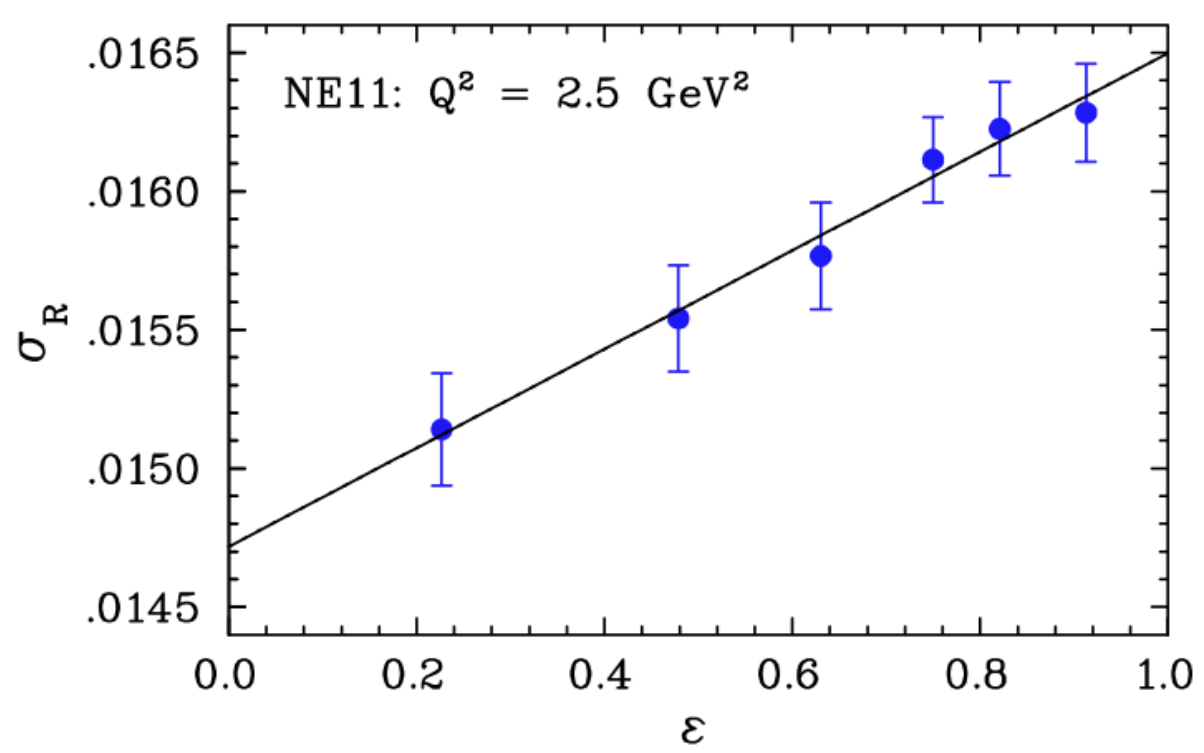

FIG. 2. Reduced cross section $\sigma_{R}$ as a function of $\varepsilon$ at $Q^{2}=2.5 \mathrm{GeV}^{2}$ [3]. Data is taken from the SLAC NE11 experiment [4]. The slope of the line equals $G_{E}^{2}$ and the intercept equals $G_{M}^{2}$.

$\varepsilon \rightarrow 0$, the cross section depends only on $G_{M}$. As $Q^{2}$ increases, $\tau$ increases and it becomes more difficult to extract $G_{E}^{2}$ with high accuracy from the measured cross sections.

Figure 3 shows $G_{M}$ and $G_{E}$ measured by the Rosenbluth separation method as a function of $Q^{2}$ normalized to the dipole form factor:

$$
G_{\mathrm{D}}=\left[1+Q^{2} / M_{D}^{2}\right]^{-2} \text { where } M_{D}^{2}=0.71 \mathrm{GeV}^{2}
$$

As shown in to Fig. 3, the uncertainties on $G_{M}$ are $\approx 1-2 \%$ for $Q^{2}>0.1 \mathrm{GeV}^{2}$. The uncertainties on $G_{E}$ are $(1-2 \%)$ at low $Q^{2},(5-10 \%)$ at intermediate $Q^{2}$ and then grow rapidly as $Q^{2}$ increases.

\subsubsection{POLARIZATION TRANSFER METHOD}

In the polarization transfer technique, a longitudinally polarized electron beam is scattered from an unpolarized proton target. The polarization of the recoil proton is measured. There are two non-zero components of recoil proton polarization: longitudinal $\left(P_{l}\right)$ and transverse $\left(P_{t}\right)$ to its momentum in the scattering plane (see 


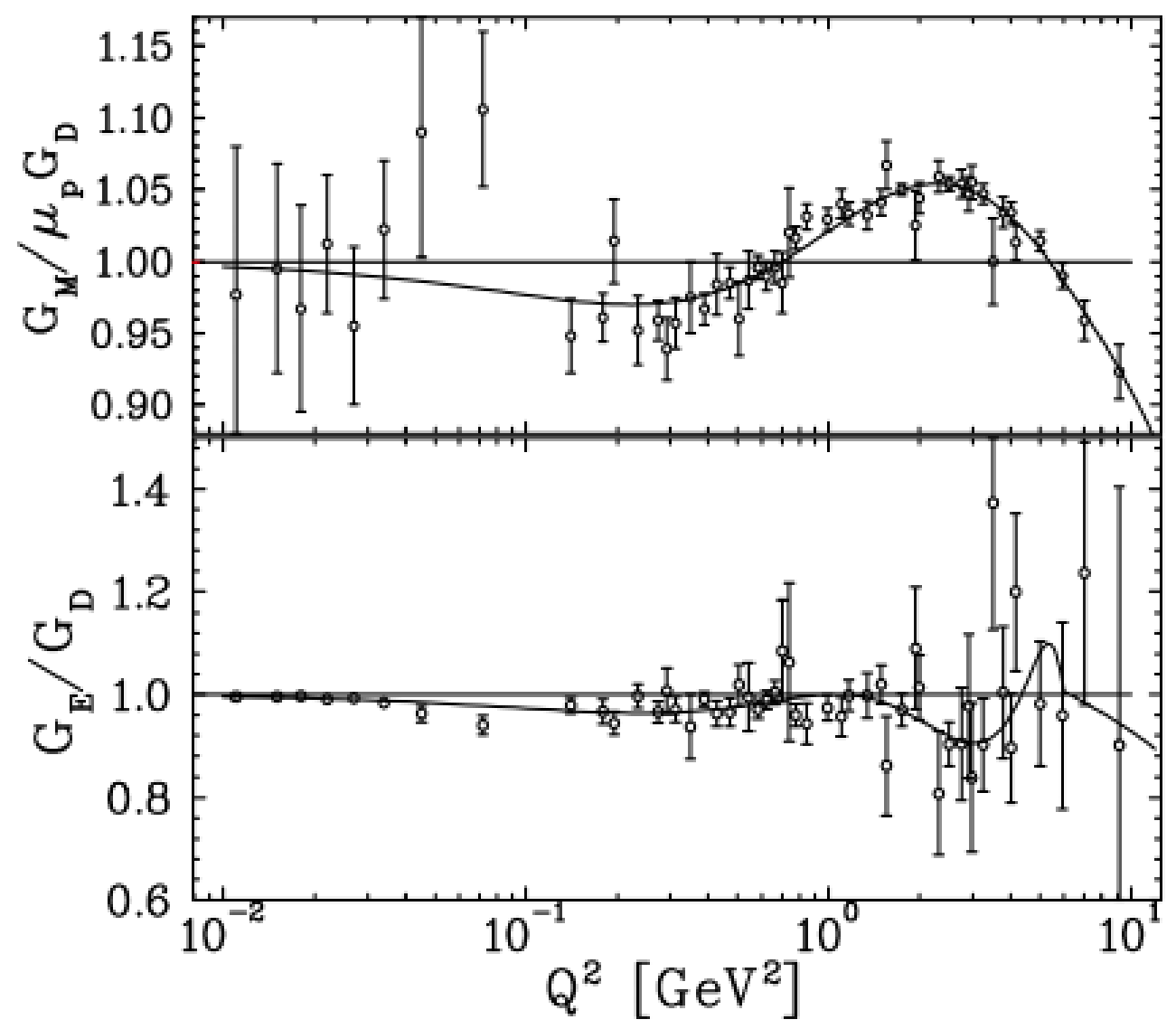

FIG. 3. Normalized $G_{M}$ (top) and $G_{E}$ (bottom) from direct Rosenbluth separation measurements as a function of $Q^{2}[5] . \mu_{p}$ is the proton magnetic moment and $G_{D}$ is the dipole form factor.

Fig. 4). The polarization component normal to the scattering plane $\left(P_{n}\right)$ is zero in the Born approximation. In the Born approximation, the two non-zero polarization components are related to the electric and magnetic proton form factors as follows $[6]$ :

$$
\begin{aligned}
& I_{0} P_{t}=-2 \sqrt{\tau(1+\tau)} G_{E} G_{M} \tan \left(\frac{\theta}{2}\right) \\
& I_{0} P_{l}=\frac{1}{M}\left(E+E^{\prime}\right) \sqrt{\tau(1+\tau)} G_{M}^{2} \tan ^{2}\left(\frac{\theta}{2}\right)
\end{aligned}
$$

where $I_{0}=G_{E}^{2}+\frac{\tau}{\varepsilon} G_{M}^{2}$. Equations (21) and (22) together give

$$
\frac{G_{E}}{G_{M}}=-\frac{P_{t}}{P_{l}} \frac{E+E^{\prime}}{2 M} \tan \left(\frac{\theta}{2}\right) .
$$




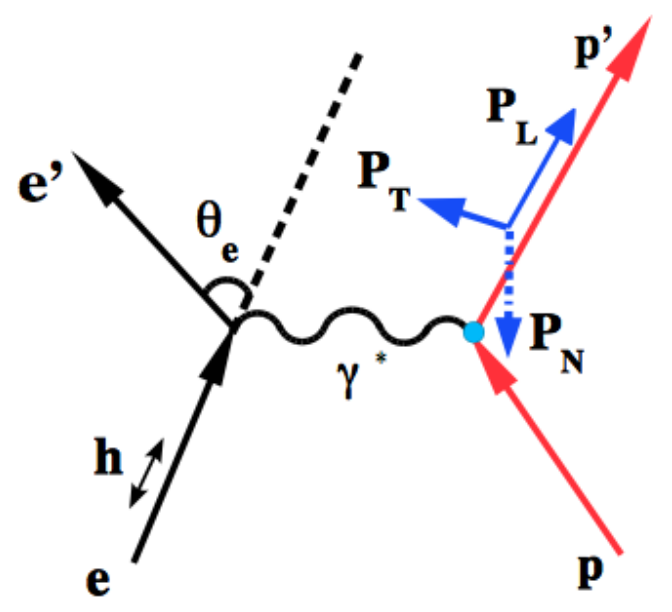

FIG. 4. Schematic diagram for the proton recoil polarization in the $\mathrm{H}\left(\vec{e}, e^{\prime} \vec{p}\right)$ reaction [3]. $P_{L}$ is the proton polarization parallel to its momentum $p^{\prime}, P_{T}$ is the proton polarization transverse to $p^{\prime}$ in the reaction plane formed by $\gamma *$ and $p^{\prime}$, and $P_{N}$ is the proton polarization normal to the reaction plane.

The proton form factor ratio, $G_{E} / G_{M}$, is obtained from a simultaneous measurement of the recoil proton polarization components using a focal plane polarimeter. Systematic uncertainties in proton form factor ratios are relatively small and radiative effects are typically a few percent [6].

Figure 5 shows the world data on $\frac{\mu_{p} G_{E}}{G_{M}}$ as determined by the Rosenbluth separation and polarization transfer techniques. More details and the comparison of the data obtained from two methods will be discussed in next section.

\subsubsection{DISCREPANCY OBSERVED BETWEEN THE TWO METHODS OF MEASUREMENTS}

The proton form factor ratio, $\frac{\mu_{p} G_{E}}{G_{M}}$, obtained from the Rosenbluth separation method is almost constant with $Q^{2}$ while the ratio obtained from the polarization transfer measurements decrease with increasing $Q^{2}$, especially for $Q^{2}>1.0 \mathrm{GeV}^{2}$. The difficulty of extracting $G_{E}$ at large $Q^{2}$ with high precision was initially suggested as the main cause of this discrepancy and was the motivation behind developing the recoil polarization technique. 


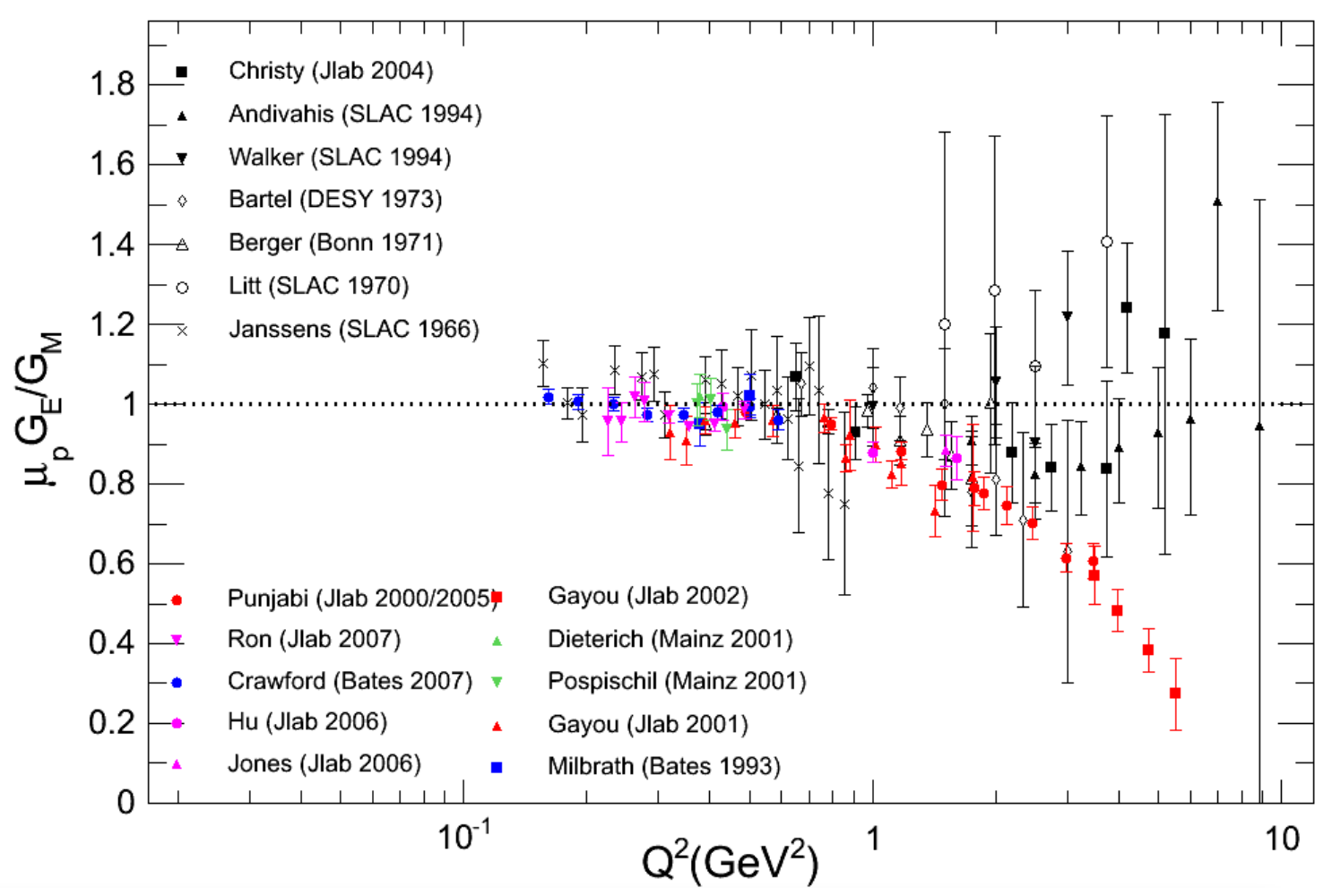

FIG. 5. The world data on the ratio of electric to magnetic form factors $\frac{\mu_{p} G_{E}}{G_{M}}$ by Rosenbluth separation method (black symbols) and polarization transfer technique (colored symbols).

In order to reduce the uncertainties of the Rosenbluth separation method at high $Q^{2}$, a "super Rosenbluth experiment" was carried out in Jefferson Lab Hall A [3]. Instead of detecting the scattered electron, they detected the recoil proton. Since the momentum of the recoil proton at fixed $Q^{2}$ is angle independent, the systematic uncertainties and several other correction terms were significantly reduced compared to the standard Rosenbluth separation method. Figure 6 shows the ratios obtained from the Super Rosenbluth technique at $Q^{2}=2.64,3.2$ and $4.1 \mathrm{GeV}^{2}$ [7] compared to the ratios obtained from the other two standard techniques. The $\mu_{p} G_{E} / G_{M}$ measurements from the super Rosenbluth separation method are as precise as the polarization transfer measurements. They agree with previous Rosenbluth measurements and therefore confirm that the discrepancy between the proton form factor ratio measurements are not due to experimental errors. 


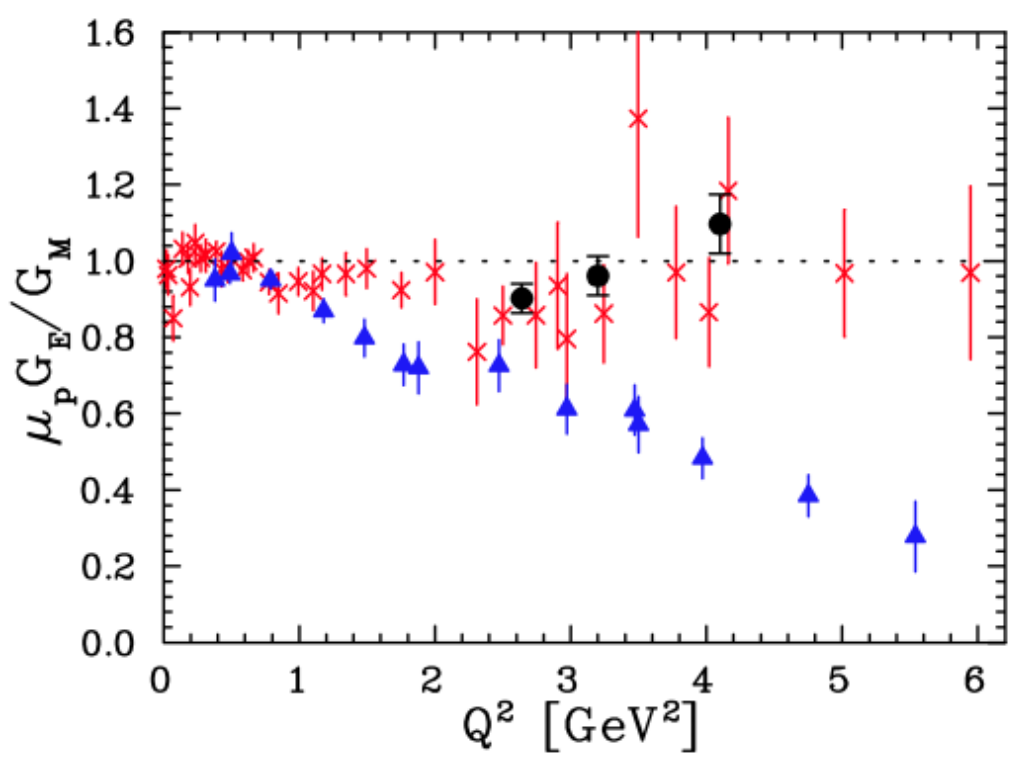

FIG. 6. Measurements of $\mu_{p} G_{E} / G_{M}$ from the super Rosenbluth separation method (circles), from previous Rosenbluth separations (red crosses), and from the polarization transfer technique (blue triangles) [7].

\subsubsection{POSSIBLE SOURCES OF DISCREPANCY}

Another possible source of the discrepancy is due to higher order corrections to cross section measurements. Figure 7 shows the difference in the reduced cross section, as a function of $\varepsilon$, found from two methods at $Q^{2}=2.64$ and $4.1 \mathrm{GeV} / \mathrm{c}^{2}$. The blue data points show the $\varepsilon$ dependence of the reduced cross section obtained from the Super Rosenbluth method. The red dashed line shows the expected slope from the polarization transfer measurements. The polarization transfer measurement slopes are arbitrarily normalized to agree with the reduced cross section at $\varepsilon=0$ [8]. The difference in the slops implies a $5-10 \% \varepsilon$-dependent correction to the cross section at these $Q^{2}$. This small correction has a large impact $(\sim 50 \%)$ on $G_{E}$ extraction.

The simple division of the reduced cross section into two terms proportional to $G_{E}^{2}$ and $G_{M}^{2}$ is only correct in the one photon exchange (first Born) approximation. The total cross section measured in the experiment includes higher order radiative processes and therefore should be corrected carefully during data analysis. Figure 8 

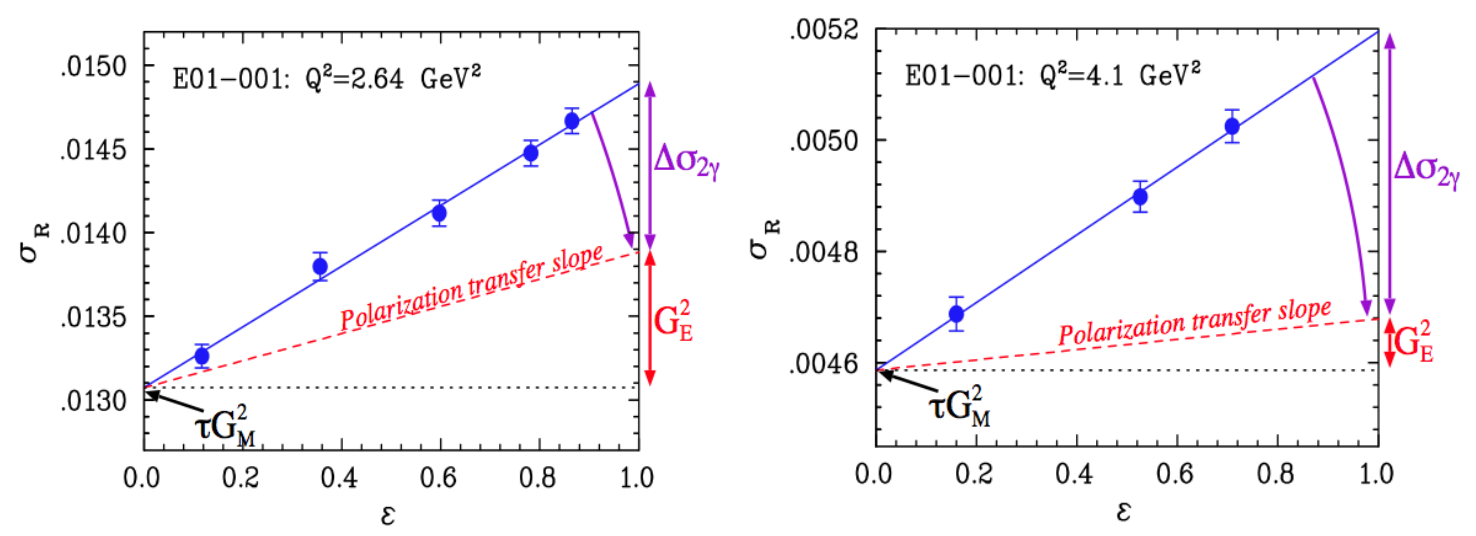

FIG. 7. The reduced cross section measured by Jefferson Lab experiment E01-001 [3] (blue) and as predicted from polarization transfer measurements (red dashed line) at $Q^{2}=2.64 \mathrm{GeV} / \mathrm{c}^{2}$ and $Q^{2}=4.1 \mathrm{GeV} / \mathrm{c}^{2}[8]$.

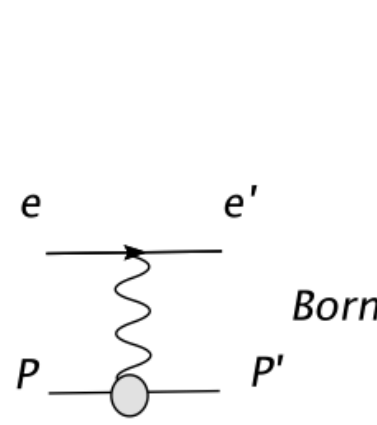

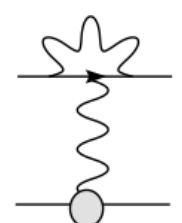

(a)

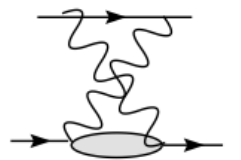

(e)

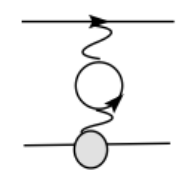

(b)

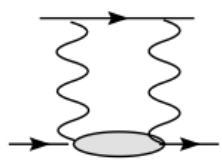

(f)

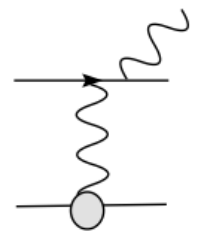

(c)

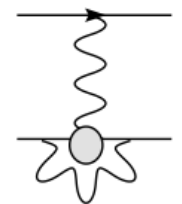

(g)

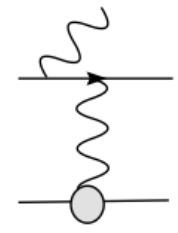

(d)

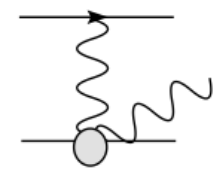

(h)

FIG. 8. Feynman diagrams of the processes associated with elastic electron-proton scattering, including the Born term, first order radiative corrections ((a) electron vertex correction, (b) vacuum polarization, (c, d) electron bremstrahlung, (g) proton vertex correction and $(\mathrm{h})$ proton bremstrahlung) and $(\mathrm{e}, \mathrm{f})$ two photon exchange.

shows the Feynman diagrams of the Born approximation term, first order radiative correction terms and higher order (two-photon) terms for elastic electron-proton scattering. 
The correction terms that involve only electron-photon vertices ( $a, b, c$ and $d$ in Fig. 8) can be calculated using QED [9] and those corrections were applied to the Rosenbluth separation and polarization transfer technique measurements. The terms that involve the proton vertex (e, f, g and h) must be estimated or measured using other techniques. The proton vertex correction was analyzed [10] and found to be small $(<0.5 \%)$ for $Q^{2}<6 \mathrm{GeV} / \mathrm{c}^{2}$. This indicates that the next leading order term, two photon exchange, could be responsible for the discrepancy in $G_{E} / G_{M}$.

The impact of the TPE correction on the Rosenbluth separation and polarization transfer measurements was calculated in Ref. [11]. They found that the TPE corrections to the polarization transfer measurements of $G_{E}^{2} / G_{M}^{2}$ are $\sim 4-5 \%$ at small $\varepsilon$ at $Q^{2} \sim 6 \mathrm{GeV} / \mathrm{c}^{2}$ and even smaller at large $\varepsilon$. Therefore the impact of TPE corrections on $G_{E} / G_{M}$ is negligible. They also found that including the TPE correction reduces $G_{E} / G_{M}$ extracted by the Rosenbluth separation method significantly. Calculations of TPE corrections and their impact on the measurements will be discussed in detail in the next chapter. 


\section{CHAPTER 2}

\section{TWO PHOTON EXCHANGE}

There have been several approaches used to calculate the TPE contribution to elastic electron-proton scattering theoretically, as well as a significant effort to measure these experimentally. This chapter will describe a few of those theoretical efforts in detail. The existing measurements of the TPE contribution by comparing the positron-proton to electron-proton elastic scattering cross section will be also discussed.

Figure 9 shows the box and crossed-box diagrams of the two photon exchange in elastic electron-proton scattering which involve the exchange of two virtual photons with an intermediate hadronic state that includes the ground state and all excited states. The TPE contribution is difficult to calculate because the intermediate hadronic state must be integrated over all baryonic resonance and continuum states that can be excited by the virtual photon. Therefore, TPE is typically neglected in calculating radiative corrections of previous Rosenbluth and the polarization transfer measurements. In order to describe the elastic scattering amplitude in the presence of TPE, one can use three form factors: $\tilde{F}_{1}, \tilde{F}_{2}$ and $\tilde{F}_{3}$. So, the electromagnetic hadron current operator $\Gamma_{\gamma}^{\mu}$ is can be rewritten as

$$
\Gamma_{\gamma}^{\mu}=\gamma^{\mu} \tilde{F}_{1}+\frac{i \sigma^{\mu \nu} q_{\nu}}{2 M} \tilde{F}_{2}+\frac{\gamma \cdot K P}{M^{2}} \tilde{F}_{3}
$$

where $K=\left(k+k^{\prime}\right) / 2$ and $P=\left(p+p^{\prime}\right) / 2 . \tilde{F}_{1}, \tilde{F}_{2}$ and $\tilde{F}_{3}$ are complex functions of $\nu$ and $Q^{2}$ where $\nu=K \cdot P$. In the one photon exchange limit, $\tilde{F}_{1,2}$ are the usual Dirac and Pauli form factors, while the new form factor $\tilde{F}_{3}$ exists only at two photon exchange level and beyond. Alternatively, the hadron current can be expressed in terms of the generalized (complex) Sachs electric and magnetic form factors, $\tilde{G}_{M}=G_{M}+\delta G_{M}$ and $\tilde{G}_{E}=G_{E}+\delta G_{E}$. The methods of evaluating the amplitudes $\tilde{F}_{3}, \delta G_{E}$ and $\delta G_{M}$ originating from the two photon exchange will be discussed in the remaining sections. 

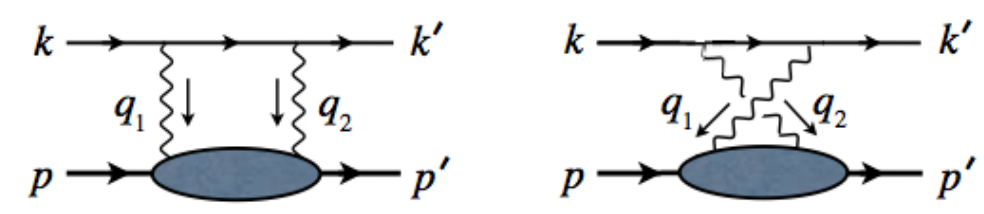

FIG. 9. Box and crossed-box two-photon exchange contributions to elastic electronnucleon scattering. The total four-momentum transfer to the nucleon is $q=k-k^{\prime}=$ $q_{1}+q_{2}$.

\subsection{PHENOMENOLOGICAL ESTIMATES}

Guichon and Vanderhaeghen [12] estimated the size of the TPE corrections to the elastic electron-proton cross section by assuming that these corrections completely reconciled the proton form factor ratios measured by the Rosenbluth and polarization transfer methods. They expressed the elastic scattering T-matrix in terms of the phenomenological form factors as

$$
T=\frac{e^{2}}{Q^{2}} \bar{u}\left(k^{\prime}\right) \gamma_{\mu} u(k) \times \bar{u}\left(p^{\prime}\right)\left(\tilde{G}_{M} \gamma^{\mu}-\tilde{F}_{2} \frac{P^{\mu}}{M}+\tilde{F}_{3} \frac{\gamma \cdot K P^{\mu}}{M^{2}}\right) u(p)
$$

where $\tilde{G}_{M}, \tilde{F}_{2}$ and $\tilde{F}_{3}$ are complex functions of $\nu$ and $Q^{2}$. In the Born approximation, these function become $\tilde{G}_{M}=G_{M}, \tilde{F}_{2}=F_{2}$ and $\tilde{F}_{3}=0$. By analogy, they defined $\tilde{G}_{E}=\tilde{G}_{M}-(1+\tau) \tilde{F}_{2}$. Using standard techniques, they calculated approximate expressions for the cross section and polarization transfer as

$$
\begin{gathered}
\frac{d \sigma}{C_{B}\left(\varepsilon, Q^{2}\right)} \simeq \frac{\left|\tilde{G}_{E}\right|^{2}}{\tau}\left\{\tau+\varepsilon \frac{\left|\tilde{G}_{E}\right|^{2}}{\left|\tilde{G}_{M}\right|^{2}}+2 \varepsilon\left(\tau+\frac{\left|\tilde{G}_{E}\right|}{\left|\tilde{G}_{M}\right|}\right) \mathcal{R} e\left(\frac{\nu \tilde{F}_{3}}{M^{2}\left|\tilde{G}_{M}\right|}\right)\right\} \\
\frac{P_{t}}{P_{l}} \simeq-\sqrt{\frac{2 \varepsilon}{\tau(1+\varepsilon)}}\left\{\frac{\left|\tilde{G}_{E}\right|}{\left|\tilde{G}_{M}\right|}+\left(1-\frac{2 \varepsilon}{1+\varepsilon} \frac{\left|\tilde{G}_{E}\right|}{\left|\tilde{G}_{M}\right|}\right) \mathcal{R} e\left(\frac{\nu \tilde{F}_{3}}{M^{2}\left|\tilde{G}_{M}\right|}\right)\right\}
\end{gathered}
$$

where $C_{B}\left(\varepsilon, Q^{2}\right)$ is a phase space factor. In order to separate the Born and the TPE correction terms, the generalized form factors were split into $\tilde{G}_{M}=G_{M}+\delta G_{M}, \tilde{G}_{E}=$ $G_{E}+\delta G_{M}$ and $\tilde{F}_{3}=\tilde{F}_{3}$. Finally, they expressed the Rosenbluth and polarization transfer measurements of the proton form factor ratio as

$$
R_{\text {Rosenbluth }}^{2}=\frac{\left|\tilde{G}_{E}\right|^{2}}{\left|\tilde{G}_{M}\right|^{2}}+2\left(\tau+\frac{\left|\tilde{G}_{E}\right|^{2}}{\left|\tilde{G}_{M}\right|^{2}}\right) Y_{2 \gamma}
$$




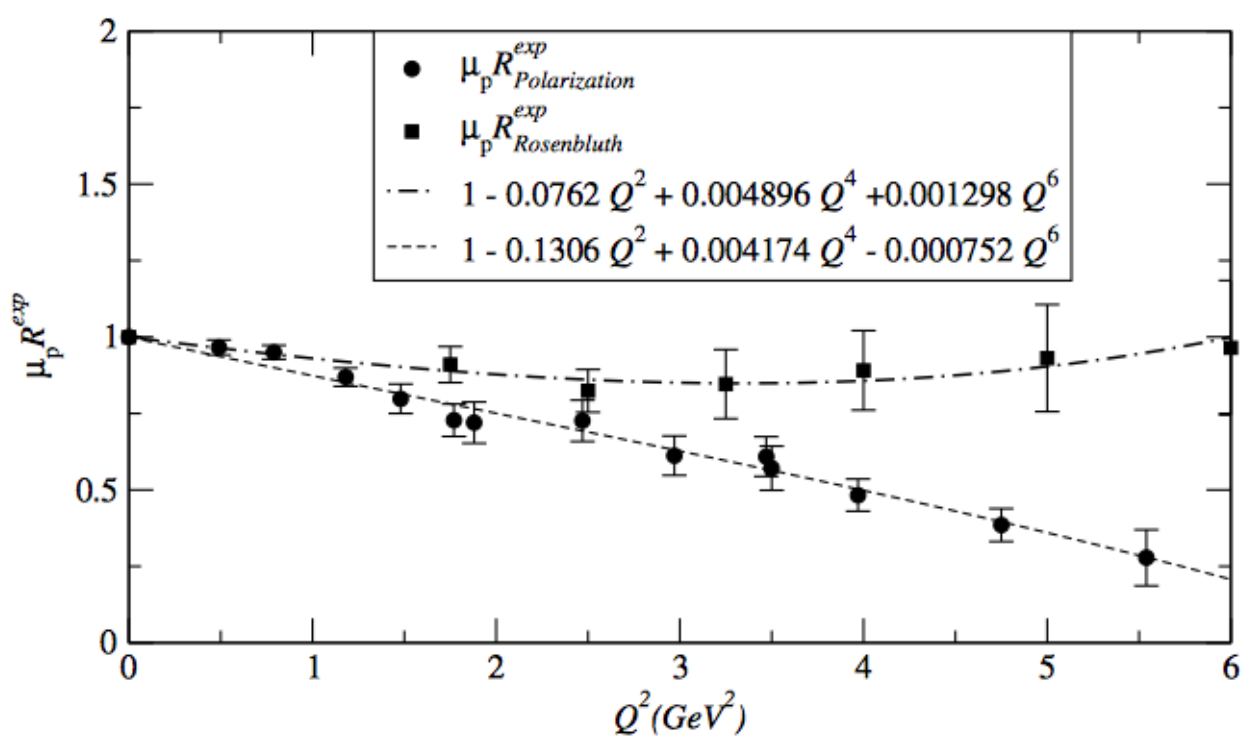

FIG. 10. The proton form factor ratio measured by the Rosenbluth method $\left(R_{\text {Rosenbluth }}^{\exp }\right)$ and the polarization transfer technique $\left(R_{\text {polarization }}^{\exp }\right)$ and their polynomial fits [12].

$$
R_{\text {polarization }}^{\exp }=\frac{\left|\tilde{G}_{E}\right|}{\left|\tilde{G}_{M}\right|}+2\left(1-\frac{2 \varepsilon}{1+\varepsilon} \frac{\left|\tilde{G}_{E}\right|^{2}}{\left|\tilde{G}_{M}\right|^{2}}\right) Y_{2 \gamma}
$$

$Y_{2 \gamma}$ is a dimensionless quantity defined as: $Y_{2 \gamma}\left(\nu, Q^{2}\right)=\mathcal{R} e\left(\frac{\nu \tilde{F}_{3}}{M^{2}\left|\tilde{G}_{M}\right|}\right)$. In the Born approximation, $\left(R_{\mathrm{Rosenbluth}}^{\exp }\right)^{2}=\frac{\left|G_{E}\right|^{2}}{\left|G_{M}\right|^{2}}$ and $R_{\text {polarization }}^{\exp }=\frac{\left|G_{E}\right|}{\left|G_{M}\right|}$.

Equations 28 and 29 were then solved numerically by fitting the data with a polynomial in $Q^{2}$ as shown in Fig. 10 and solving for the ratio $Y_{2 \gamma}$ is shown in Fig. 11. These ratios are independent of $\varepsilon$ and very small (on the order of a few percent), which is consistent with the expectations for the size of the effective two photon correction. They also compared the proton form factor ratios obtained from the Rosenbluth method and polarization transfer technique with the corrected ratio ( $\left.R^{\exp }=\left|\tilde{G}_{E}\right| /\left|\tilde{G}_{M}\right|\right)$ and found that the ratio extracted from the polarization method is only slightly changed by the two-photon correction (see Fig. 12).

A similar analysis was performed by Arrington [13] to extract $\delta G_{E, M}$ and $Y_{2 \gamma}$. He combined data from Rosenbluth, polarization transfer, and positron-proton scattering measurements to constrain the two-photon amplitudes. 


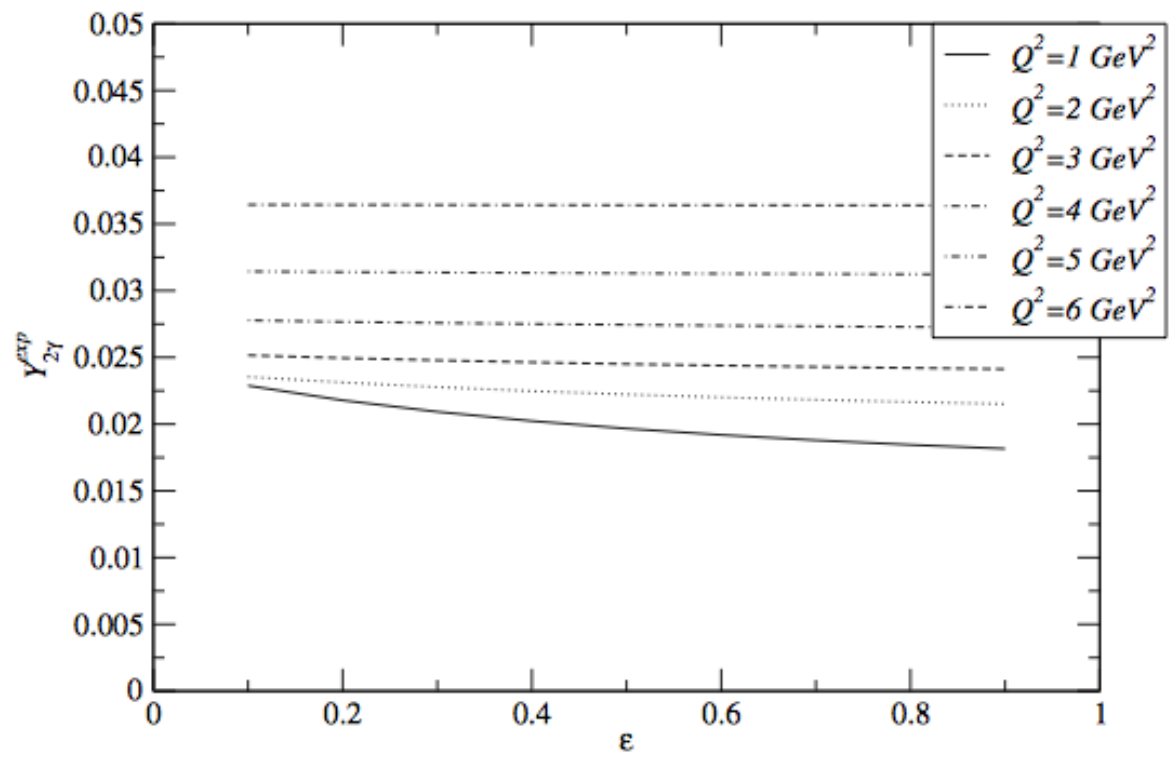

FIG. 11. The ratio $Y_{2 \gamma}$ as a function of $\varepsilon$ for different $Q^{2}$ from the phenomenological extraction by Guichon and Vanderhaeghen.

\subsection{THEORETICAL CALCULATIONS}

\subsubsection{HADRONIC CALCULATION}

Blunden, Melnitchouk and Tjon [11] calculated the TPE contribution by considering the two photon exchange box and crossed-box amplitudes with an intermediate nucleon (see Fig. 9). According to their calculation, the total TPE amplitude

$$
\mathcal{M}_{\gamma \gamma}=\mathcal{M}_{\gamma \gamma}^{\mathrm{box}}+\mathcal{M}_{\gamma \gamma}^{\mathrm{xbox}}
$$

with

$$
\mathcal{M}_{\gamma \gamma}^{\text {box }(\text { xbox })}=-i e^{4} \int \frac{d^{4} q_{1}}{(2 \pi)^{4}} L_{\mu \nu}^{\text {box (xbox })} H_{N}^{\mu \nu} \Delta_{F}\left(q_{1}, \lambda\right) \Delta_{F}\left(q_{2}, \lambda\right)
$$

where $L_{\mu \nu}^{\mathrm{box}(\mathrm{xbox})}$ is the box (crossed-box) leptonic tensor and $H_{N}^{\mu \nu}$ is the electromagnetic nucleon elastic hadronic tensor.

$$
\begin{aligned}
L_{\mu \nu}^{\mathrm{box}} & =\bar{u}_{e}\left(k^{\prime}\right) \gamma_{\mu} S_{F}\left(k-q_{1}, m_{e}\right) \gamma_{\nu} u_{e}(k) \\
L_{\mu \nu}^{\mathrm{xbox}} & =\bar{u}_{e}\left(k^{\prime}\right) \gamma_{\nu} S_{F}\left(k-q_{2}, m_{e}\right) \gamma_{\mu} u_{e}(k)
\end{aligned}
$$




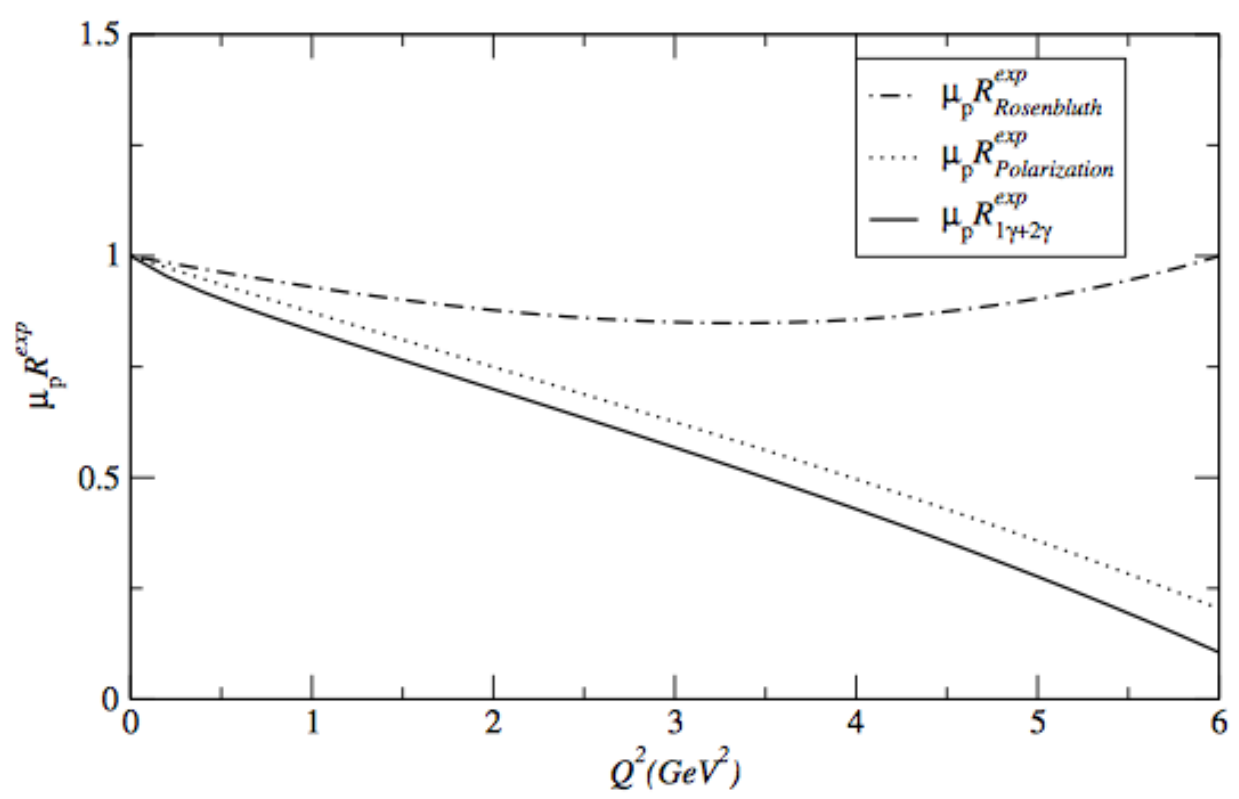

FIG. 12. The original proton from factor ratios $\left(\mu_{p} G_{E} / G_{M}\right)$ measured by Rosenbluth method and polarization transfer technique compared with the TPE corrected ratio $\left(\mu_{p} R_{1 \gamma+2 \gamma}^{e x p}\right)$ extracted using phenomenological estimates [12].

$$
H_{N}^{\mu \nu}=\bar{u}_{N}\left(p^{\prime}\right) \Gamma_{\gamma}^{\mu} S_{F}\left(p+q_{1}, M\right) \Gamma_{\gamma}^{\nu} u_{N}(p)
$$

where the electromagnetic current operator $\Gamma_{\gamma}^{\mu}$ is given in Eq. (7). The lepton and photon propagators are given by

$$
S_{F}(k, m)=\frac{(k+m)}{k^{2}-m^{2}+i \epsilon}, \quad \text { and } \quad \Delta_{F}(k, \lambda)=\frac{1}{k^{2}-\lambda^{2}+i \epsilon},
$$

respectively. Note that the above conventions follow Ref. [8]. The relative correction to the elastic Born cross section, $\delta$, is given by

$$
\delta=\frac{2 \mathcal{R} e\left(\mathcal{M}_{\gamma}^{*} \mathcal{M}_{\gamma \gamma}\right)}{\left|\mathcal{M}_{\gamma}\right|^{2}}
$$

Figure 13 shows the difference between the full TPE correction to the elastic cross section by hadronic calculation $\left(\delta_{\text {Full }}\right)$ and the commonly used radiative correction from Mo and Tsai $\left(\delta_{\mathrm{MT}}\right)[9], \bar{\delta}=\delta_{\text {Full }}-\delta_{\mathrm{MT}}$. The additional corrections from TPE are most significant at low $\varepsilon$ and vanish at large $\varepsilon$. The corrections are approximately linear with $\varepsilon$ at low $Q^{2}$, but become increasingly nonlinear as $Q^{2}$ increases. 


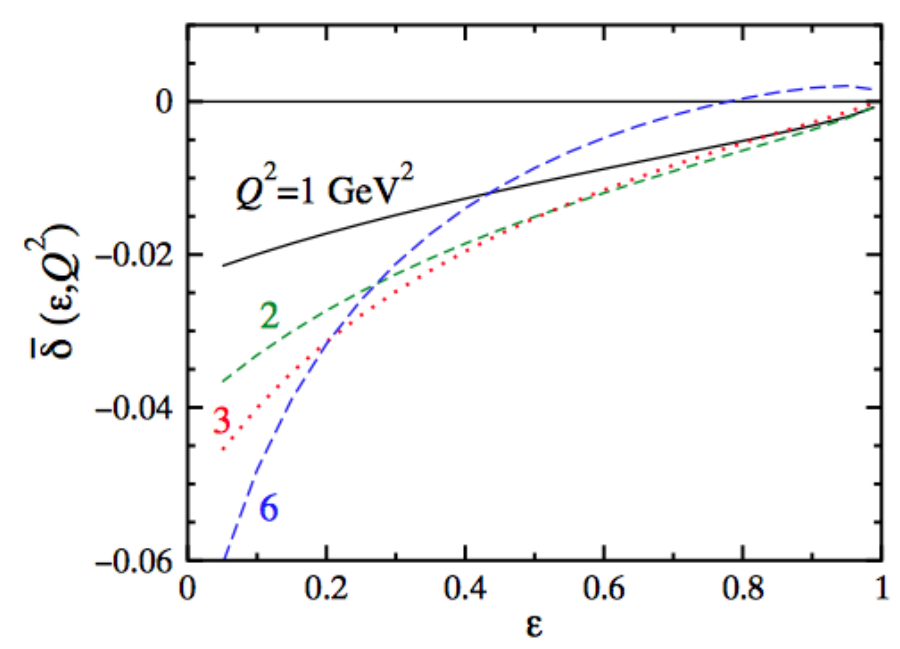

FIG. 13. The difference between the full two-photon exchange correction to the elastic cross section from the hadronic calculation [11] and the commonly used radiative correction from Mo and Tsai [9] for $Q^{2}=1-6 \mathrm{GeV}^{2}$. The numbers labeling the curves denote the respective $Q^{2}$ values in $\mathrm{GeV}^{2}$.

Hadronic calculations were later extended to include the other heavier nucleon resonances such as $\mathrm{P}_{33}(\Delta), \mathrm{D}_{13}, \mathrm{D}_{33}, \mathrm{P}_{11}, \mathrm{~S}_{11}$ and $\mathrm{S}_{31}$ as the intermediate states [14, 15]. Figure 14 shows the calculated TPE corrections to the reduced cross section with and without including those heavier resonances. In general, each resonance two-photon correction is proportional to a sum of squares of the nucleon-photon coupling constants of that resonance. These coupling constants are not well known. At low to moderate $Q^{2}$, the TPE corrections are determined mainly by the nucleon and $\mathrm{P}_{33}$ intermediate states and therefore one does not have to consider the coupling constants of the other resonances to get a good estimate of the overall two-photon exchange effect.

\subsubsection{PARTONIC CALCULATION}

Chen, Afanasev, Brodsky, Carlson and Vanderhaeghen calculated the TPE contribution to elastic electron-nucleon scattering at large momentum transfer through the scattering off partons (quarks) in a nucleon $[16,17]$. They related the process on the nucleon to generalized parton distributions (GPDs) and this approach effectively summed all possible excitations of inelastic nucleon intermediate states. In order 


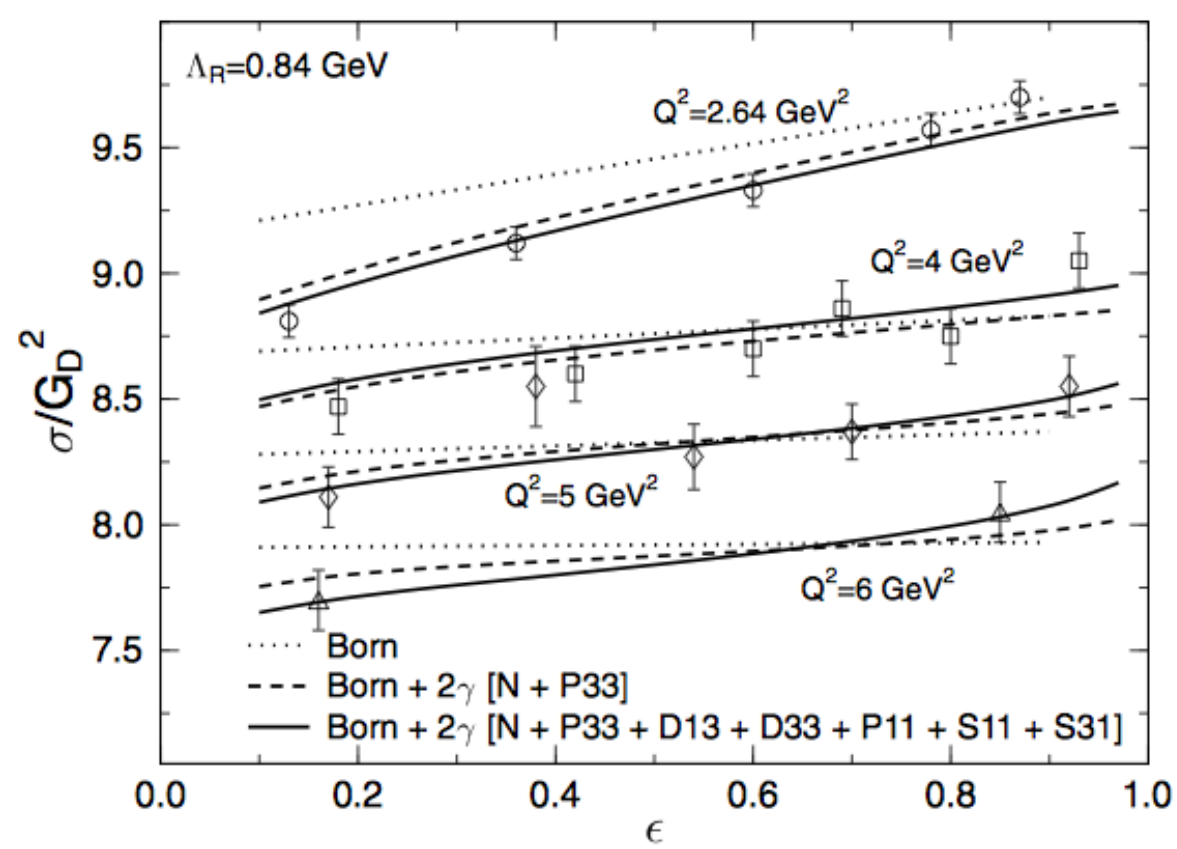

FIG. 14. The reduced cross section, scaled by the dipole form factor $\left(G_{D}\right)$, including the effects of adding TPE corrections to the Born cross section [15]. The Born cross section is calculated using $G_{E} / G_{M}$ measured by the polarization technique. The intermediate state includes a nucleon and the indicated hadron resonances. The curves for $Q^{2}=2.64,4$ and $6 \mathrm{GeV}^{2}$ have been shifted vertically by $-0.04,+0.04$ and +0.09 , respectively, for clarity. The data points at the four $Q^{2}$ values are taken from $[3]$.

to calculate the TPE contributions at large $Q^{2}$, they considered the lepton-quark scattering process represented by $H$ in Fig. 15 and also assumed that both of the virtual photons interact with the same quark in the proton.

In their work, elastic lepton-quark scattering

$$
l(k)+q\left(p_{q}\right) \rightarrow l\left(k^{\prime}\right)+q\left(p_{q}^{\prime}\right)
$$

is described by two independent kinematic invariants, $\hat{s} \equiv\left(k+p_{q}\right)^{2}$ and $Q^{2}=-t=$ $-\left(k-k^{\prime}\right)^{2}$. A crossing variable, $\hat{u} \equiv\left(k-p_{q}^{\prime}\right)$ is also introduced. The reduced cross 


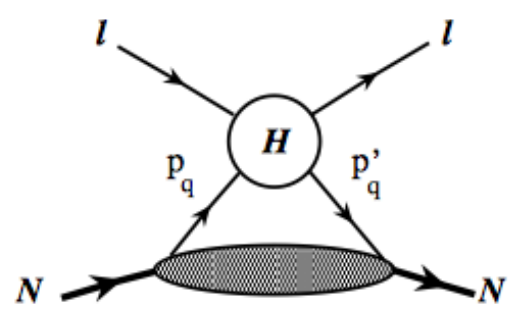

FIG. 15. The handbag approximation for electron-nucleon elastic scattering. $H$ represents the lepton scatters from the quarks in the nucleon and the lower blob represents the GPD's of the nucleon.

section, including corrections up to order $e^{2}$ can be written as

$$
\begin{aligned}
\sigma_{R}=G_{M}^{2} & +\frac{\varepsilon}{\tau} G_{E}^{2}+2 G_{M} \mathcal{R} e\left(\delta \tilde{G}_{M}+\varepsilon \frac{\nu}{M^{2}} \tilde{F}_{3}\right) \\
& +2 \frac{\varepsilon}{\tau} G_{E} \mathcal{R} e\left(\delta \tilde{G}_{E}+\frac{\nu}{M^{2}} \tilde{F}_{3}\right)+\mathcal{O}\left(e^{4}\right)
\end{aligned}
$$

The TPE form factors, $\delta \tilde{G}_{M}, \delta \tilde{G}_{E}$ and $\tilde{F}_{3}$, were then expressed in terms of three integrals containing GPDs [16]

$$
\begin{gathered}
\delta \tilde{G}_{M}=C \\
\delta \tilde{G}_{E}=-\left(\frac{1+\varepsilon}{2 \varepsilon}\right)(A+C)+\sqrt{\frac{1+\varepsilon}{2 \varepsilon}} B \\
\tilde{F}_{3}=\frac{M^{2}}{\nu}\left(\frac{1+\varepsilon}{2 \varepsilon}\right)(A-C)
\end{gathered}
$$

where

$$
\begin{gathered}
A \equiv \int_{-1}^{1} \frac{d x}{x} \frac{(\hat{s}-\hat{u})\left(\tilde{f}_{1}^{\text {hard }}-\hat{s} \hat{u} \tilde{f}_{3}\right)}{(s-u)} \sum_{q} e_{q}^{2}\left(H^{q}+E^{q}\right) \\
B \equiv \int_{-1}^{1} \frac{d x}{x} \frac{(\hat{s}-\hat{u})\left(\tilde{f}_{1}^{\text {hard }}-\hat{s} \hat{u} \tilde{f}_{3}\right)}{(s-u)} \sum_{q} e_{q}^{2}\left(H^{q}-\tau E^{q}\right) \\
C \equiv \int_{-1}^{1} \tilde{f}_{1}^{\text {hard }} \operatorname{sgn}(x) \sum_{q} e_{q}^{2} \tilde{H}^{q}
\end{gathered}
$$

The integrals depend on the hard scattering kernels $\left(\tilde{f}_{1}, \tilde{f}_{3}\right)$ and the generalized parton distributions $H^{q}, E^{q}$ and $\tilde{H}^{q}$ which describe removing a quark of a certain momentum from a hadron and replacing it with a quark of another momentum. 


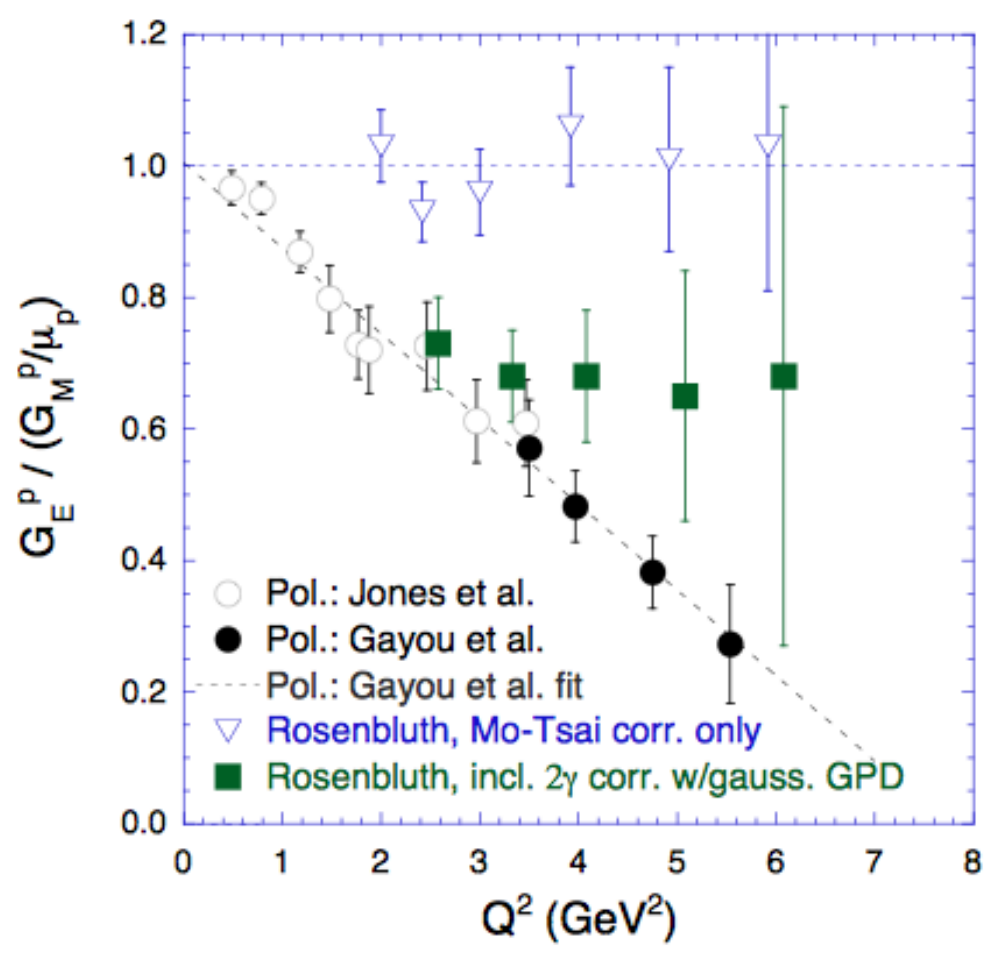

FIG. 16. The ratio of $\mu_{p} G_{E} / G_{M}$ as measured by Rosenbluth separation (hollow triangles) [4], as measured by polarization transfer technique (circles) [6, 18] and as measured by Rosenbluth and corrected for TPE effects in the partonic model (solid squares) [17].

The GPDs were estimated using two different models : a gaussian parametrization and a modified Regge parametrization. Figure 16 shows the Rosenbluth data by Andivahis et al. [4] without and with TPE corrections calculated using the partonic model compared to the polarization transfer measurements. At $Q^{2}=2-3 \mathrm{GeV}^{2}$, the inclusion of TPE corrections leads to a good agreement between Rosenbluth and polarization transfer measurements. At higher $Q^{2}$, there is only partial reconciliation between two methods.

\subsubsection{QCD APPROACH}

Another method of calculating the TPE corrections to the elastic electron-proton amplitude at large $Q^{2}$ is by calculating its leading order perturbative QCD (pQCD) contribution [19]. According to Fig. 17, the pQCD contribution to the TPE exchange 


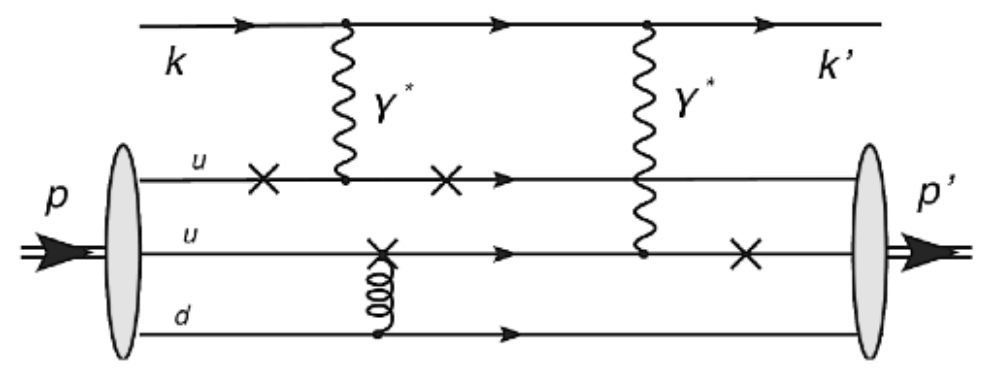

FIG. 17. Electron-proton elastic scattering with two hard photon exchange. Other possibilities to attach the gluon are indicated by crosses and the third quark is conventionally chosen as the $d$ quark [19].

can be given by a convolution of integrals of proton distribution amplitudes (DAs).

The $T$-matrix for elastic ep scattering can be expressed through three independent Lorentz structures as

$$
T_{h, \lambda_{N}^{\prime}, \lambda_{N}}=\frac{e^{2}}{Q^{2}} \bar{u}\left(k^{\prime}, h\right) \gamma_{\mu}(k, h) \times \bar{u}\left(p^{\prime}, \lambda_{N}^{\prime}\right)\left(\tilde{G}_{M} \gamma^{\mu}-\tilde{F}_{2} \frac{P^{\mu}}{M}+\tilde{F}_{3} \frac{\gamma \cdot K P^{\mu}}{M^{2}}\right) u\left(p, \lambda_{N}\right)
$$

where $h= \pm 1 / 2$ is the electron helicity and $\lambda_{N}$ and $\lambda_{N}^{\prime}$ are the helicities of the incoming and outgoing proton, respectively. $\tilde{G}_{M}, \tilde{F}_{2}, \tilde{F}_{3}$ are complex functions of $\nu$ and $Q^{2}$ and their $1 \gamma$ and $2 \gamma$ contributions can be separated as $\tilde{G}_{M}=G_{M}+\delta \tilde{G}_{M}$ and $\tilde{F}_{2}=F_{2}+\delta \tilde{F}_{2}$. Kivel and Vanderhaeghen expressed these leading TPE amplitudes in terms of the leading twist proton DAs at large $Q^{2}$. According to their work in [19], the leading behavior for $\delta \tilde{G}_{M}$ and $\frac{\nu}{M^{2}} \tilde{F}_{3}$ goes as $1 / Q^{4}$. They also found that $\delta \tilde{F}_{2}$ decreases as $1 / Q^{6}$ and is therefore suppressed at large $Q^{2}$. In order to evaluate the integrals, Kivel and Vanderhaeghen used two different models for the DAs: COZ and BLW. More details of these models can be found in Ref. [19].

The results of the pQCD calculation are shown in Fig. 18. The inclusion of TPE noticeably changes the slope of the Rosenbluth plots and quantitatively the TPE correction calculated with the COZ model is nearly twice as large as with the BLW model. They also predicted $R=\sigma\left(e^{+} p\right) / \sigma\left(e^{-} p\right)$ using pQCD calculations and found that there will be $25 \%(\mathrm{BLW})$ to $5 \%(\mathrm{COZ})$ deviation from unity for those ratios. Borisyuk and Kobushkin [21] also used the pQCD approach to analyze the TPE effect and found comparable results. 

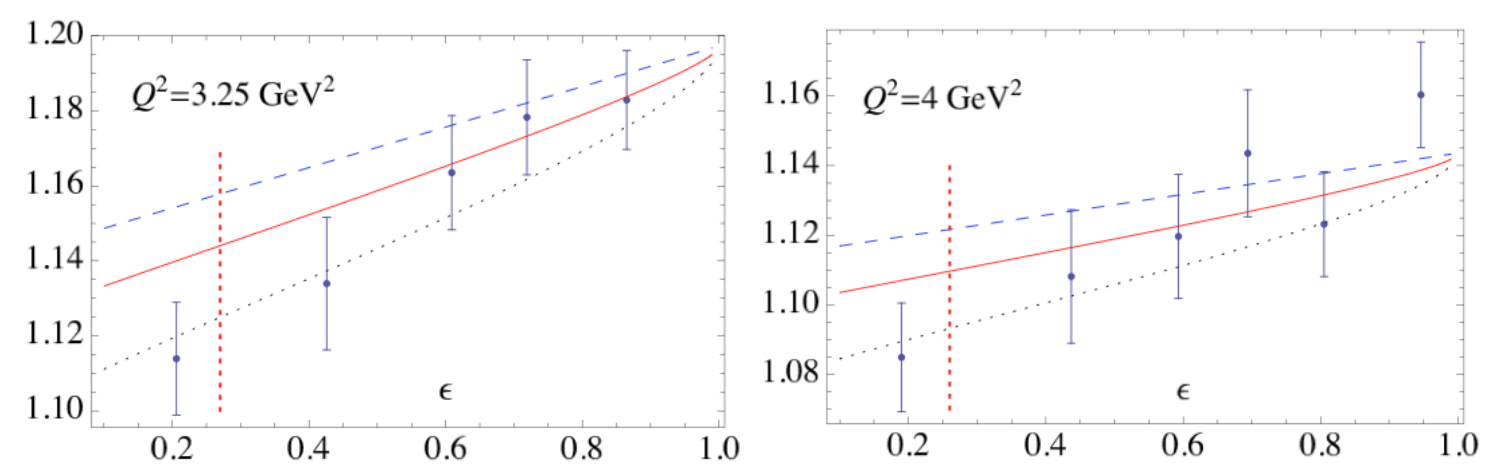

FIG. 18. $\frac{\sigma_{R}}{\left(\mu_{p} G_{D}\right)^{2}}$ as a function of $\varepsilon$ at $Q^{2}=3.25$ and $4.0 \mathrm{GeV}^{2}$. Data points are from Rosenbluth measurements by Andivahis et al. [4]. The blue dashed line represents the Born approximation cross section calculated with polarization transfer form factors from $[6,20,18]$. The solid red and dotted black curves show the inclusion of the TPE contribution calculated in the pQCD approach with BLW and COZ models for the proton distribution amplitudes, respectively. The vertical dotted line indicates the epsilon value above which their description for hard photons is valid [19].

\subsubsection{SUMMARY}

The calculation of the TPE contribution to elastic electron-proton scattering were performed in hadronic and partonic frameworks. The hadronic calculations are limited to relatively low $Q^{2}\left(\sim Q^{2}<3 \mathrm{GeV}^{2}\right)$ and the partonic calculations are valid only at high $Q^{2}$.

Even though these models are valid at different regions and their results for the TPE contribution are quantitatively different, they all predict that the TPE effect is small at larger $\varepsilon$ and weakly depends on $Q^{2}$. Those TPE calculations resolve only a part of the observed form factor discrepancy and therefore indicate the need for precise, model-independent TPE measurements to identify whether TPE is the cause of the proton form factor discrepancy.

\subsection{TPE MEASUREMENTS}

The TPE contribution to the elastic electron-nucleon scattering can be directly measured by a precise comparison of positron-proton and electron-proton elastic cross sections. The experimental techniques and data from previous experiments will be 
discussed in this section.

\subsubsection{EXPERIMENT TECHNIQUE}

The total amplitude of the elastic lepton-proton scattering can be written down as the summation of the Born term and first order corrections shown in Fig. 8.

$$
A_{e p \rightarrow e p}=q_{e} q_{p}\left[A_{\text {Born }}+q_{e}^{2} A_{\text {e.vertex }}+q_{p}^{2} A_{\text {p.vertex }}+q_{e}^{2} A_{\text {loop }}+q_{e} q_{p} A_{2 \gamma}+q_{e} A_{\text {e.br }}+q_{p} A_{\text {p.br }}\right]
$$

where $q_{e}$ and $q_{p}$ are the charges of the lepton and proton. $A_{\text {Born }}, A_{\text {e.vertex }}, A_{\text {p.vertex }}$, $A_{\text {loop }}$ and $A_{2 \gamma}$ represents the amplitudes of one photon exchange, electron vertex, proton vertex, loop diagram and two photon exchange, respectively. By squaring the amplitudes of Eq. (46) and keeping only the corrections up to order $\alpha$, we get

$$
\left|A_{e p \rightarrow e p}\right|^{2} \approx e^{4}\left[A_{\text {Born }}^{2}+2 e^{2} A_{\text {Born }} \mathcal{R} e\left(A_{\text {loop }+ \text { vertex }}\right)+2 q_{e} q_{p}\left(A_{\text {Born }} \mathcal{R} e\left(A_{2 \gamma}\right)+A_{\text {e.br }}^{*} A_{\text {p.br }}\right)\right]
$$

Here $q_{e}^{2}$ and $q_{p}^{2}$ are replaced by $e^{2}$. The interference term between the Born amplitude and the sum of loop and vertex amplitudes $\left(A_{\text {Born }} \mathcal{R} e\left(A_{\text {loop+vertex }}\right)\right)$ appears in even powers of $e$, and is therefore identical for positron-proton and electron-proton scattering. The imaginary part of $A_{2 \gamma}$ is negligible compared to the Born term and typically neglected. The interference between the Born and bremsstrahlung terms is zero because they have different final states. The ratio of the $e^{+} p$ and $e^{-} p$ cross sections can then be written as

$$
\begin{aligned}
R & =\frac{\sigma\left(e^{+} p\right)}{\sigma\left(e^{-} p\right)} \approx \frac{1+\delta_{\text {even }}-\delta_{2 \gamma}+\delta_{\text {e.p.br }}}{1+\delta_{\text {even }}+\delta_{2 \gamma}-\delta_{\text {e.p.br }}} \\
& \approx 1-2\left(\delta_{2 \gamma}+\delta_{\text {e.p.br }}\right) /\left(1+\delta_{\text {even }}\right)
\end{aligned}
$$

where $\delta_{\text {even }}=2 e^{2} A_{\text {Born }} \mathcal{R} e\left(A_{\text {loop+vertex }}\right) / A_{\text {Born }}^{2}$ is the total charge-even radiative correction factor, $\delta_{2 \gamma}$ and $\delta_{\text {e.p.br }}$ are the fractional TPE and lepton-proton bremsstrahlung interference contributions. $\delta_{\text {even }}$ is typically small and neglected in most previous extractions [22] and $\delta_{\text {e.p.br }}$ is taken into account as a correction. Thus the TPE contribution can be isolated as

$$
R \approx 1-2 \delta_{2 \gamma}
$$

In the next section, we will discuss the measurements of $R$ (charge asymmetry cross section ratio) obtained from several experiments. 


\subsubsection{EXISTING DATA}

\section{Yount and Pine, 1962}

The experiment was carried out at the Stanford Mark III linear accelerator [23]. The primary electron beam passed through a 3.2 radiation length tantalum radiator to produce positrons via pair production. The remainder of the accelerator was used to accelerate the low energy positrons up to $300 \mathrm{MeV}$. Electrons were obtained by withdrawing the radiator and leaving the phasing unchanged so that electrons were accelerated to $600 \mathrm{MeV}$ and then decelerated to $300 \mathrm{MeV}$. The lepton beams then interacted with a liquid hydrogen target and the scattered events were detected by two plastic scintillators. The quantity,

$$
R=\frac{\sigma_{-}-\sigma_{+}}{\sigma_{-}+\sigma_{+}}
$$

was compared at $200 \mathrm{MeV}$ and $300 \mathrm{MeV}$ for $Q^{2}=0.012-0.2 \mathrm{GeV}^{2}$. Here $\sigma_{-}$and $\sigma_{+}$ are the differential scattering cross sections for electron-proton and positron-proton at identical angles and energies. The results showed no difference in cross sections and hence verified the first Born approximation form factor analysis in the range of $Q^{2}<0.2 \mathrm{GeV}^{2}$. See Figs. 19 and 20 .

\section{Browman et al., 1965}

The experimental method of Yount and Pine [23] was repeated at the Stanford Mark III linear accelerator with a maximum energy of $850 \mathrm{MeV}$ [24] and a $30 \mathrm{~cm}$ liquid hydrogen target. The recoiling protons and leptons in coincidence were counted by "open" counters and a Cerenkov counter in the beam line was used for counting rate corrections. The data obtained at the highest $Q^{2}$ point in this experiment was badly contaminated by unwanted background, so this point was remeasured by a counter telescope located at the focal plane of a magnetic spectrometer.

The ratio, $R=\sigma_{+} / \sigma_{-}$was measured at four different beam energies (ranging from $600-850 \mathrm{MeV}$ ) with five different scattering angles (ranging from $50-100^{\circ}$ ) and therefore covered a wide range of $\varepsilon$ at low $Q^{2}$. The ratio $R$ measured from the experiment is shown in Figs. 19 and 20. 


\section{Anderson et al., 1966}

In this experiment, the leptons (positrons or electrons) were produced by pair production in a lead radiator in the photon beam of the Cornell $2 \mathrm{GeV}$ synchrotron $[25,26]$. The target was $45 \mathrm{~cm}$ liquid hydrogen. The paths of the scattered leptons and recoil protons were detected by pairs of spark chambers placed symmetrically on both sides of the beam line. Elastic scattering events were identified by requiring coplanarity and a fit to the scattering kinematics. Data were collected at low beam energies $1.2 \mathrm{GeV}$ and $0.8 \mathrm{GeV}$.

The ratio $R=\sigma_{+} / \sigma_{-}$was measured for $Q^{2}$ between 0.3 and $1 \mathrm{GeV}^{2}$. The results showed no dependence on $Q^{2}$ and showed that the two scattering cross sections were the same within experimental errors after radiative corrections. See Figs. 19 and 20.

\section{Bartel et al., 1967}

Bartel et al. [27] used the $6 \mathrm{GeV}$ electron beam of the synchrotron at DESY, Hamburg to measure the ratio of the elastic scattering cross section for positrons and electrons on protons. A $1.5 \mathrm{~cm}$ thick $\mathrm{Cu}$ radiator was used to produce the lepton beams and those beams were then scattered from a $30 \mathrm{~cm}$ long hydrogen target. The detector system included a hodoscope, scintillation counters and a Cherenkov counter.

The ratio of cross-sections, $R=\sigma_{+} / \sigma_{-}$, was measured at two points, one at $Q^{2}=0.45 \mathrm{GeV}^{2}$ and a scattering angle of $17.5^{\circ}$, and the other at $Q^{2}=1.36 \mathrm{GeV}^{2}$ and a scattering angle of $35^{\circ}$. Their results are consistent with $R=1$ at both $Q^{2}$ values within the experimental errors and show no TPE correction to the elastic electron-proton scattering measurements.

\section{Bouquet et al., 1968}

Bouquet et al. [28] measured the positron to electron elastic scattering ratio for backward scattered leptons in coincidence with a forward recoil proton in order to decrease the background. Lepton beams were scattered from a liquid hydrogen target and the cross section measurements were taken at $\theta=180^{\circ}$ at $Q^{2}=0.3$ and $1.2 \mathrm{GeV}^{2}$. Due to the high background produced by the positron beam hitting the spectrometer, the error in the positron cross section was increased. The results were $R=1.036 \pm 0.018$ at $Q^{2}=0.3 \mathrm{GeV}^{2}$ and $R=1.076 \pm 0.046$ at 
$Q^{2}=1.2 \mathrm{GeV}^{2}$. See Figs. 19 and 20 .

\section{Mar et al., 1968}

The positron and electron beams were produced in this experiment [29] by passing a $5.5 \mathrm{GeV}$ electron beam into a water-cooled copper radiator positioned along the Stanford Linear Accelerator Center (SLAC) accelerator. The low energy electrons or positrons emerging from the radiator were accelerated to form the beams for the experiment. The SLAC $8 \mathrm{GeV}$ magnetic spectrometer was used to analyze particles scattered from a $27 \mathrm{~cm}$ diameter vertical cylinder of liquid hydrogen.

The ratio $R$ was measured at $\theta=12.5-35^{\circ}$ at an incident lepton energy of $4 \mathrm{GeV}$ and at $\theta=2.6-15.0^{\circ}$ at an incident lepton energy of $10 \mathrm{GeV}$. This extended the world TPE measurements to higher $Q^{2}$ and to smaller scattering angles. The results were consistent with $R=1$ for $Q^{2}=0.2-5.0 \mathrm{GeV}^{2}$ after radiative corrections.

\section{Summary}

The existing $R$ measurements described above were reexamined by Arrington [5]. Figure 19 shows those ratios of positron-proton to electron-proton elastic cross sections as a function of $Q^{2}$. Ratios at large $Q^{2}$ have large uncertainties and there is only slight evidence of $Q^{2}$ dependence. Arrington fitted those data with $R=a+b Q^{2}$ and yielded $b=0.0085 \pm 0.0063$, less that 1.5 standard deviations from zero. The average of the ratios is $R=1.003 \pm 0.005$. Figure 20 shows $R$ as a function of $\varepsilon$. The slope of the linear fit was $-(5.7 \pm 1.8)$ with $\chi^{2} /$ d.f $=11.1 / 22$. The low $\chi^{2}$ indicates that the uncertainties are possibly overestimated and there could be a more significant TPE effect than indicated by the fit uncertainty.

There were only four data points above $Q^{2}=2 \mathrm{GeV}^{2}$ by Mar et al. and therefore it is difficult to make a strong conclusion of TPE effects in that $Q^{2}$ region. In addition to those early measurements, three experiments were carried out recently to measure the positron-proton to electron-proton scattering cross section ratios at wide $\varepsilon$ and $Q^{2}$ range. Those three experiments are described below. 


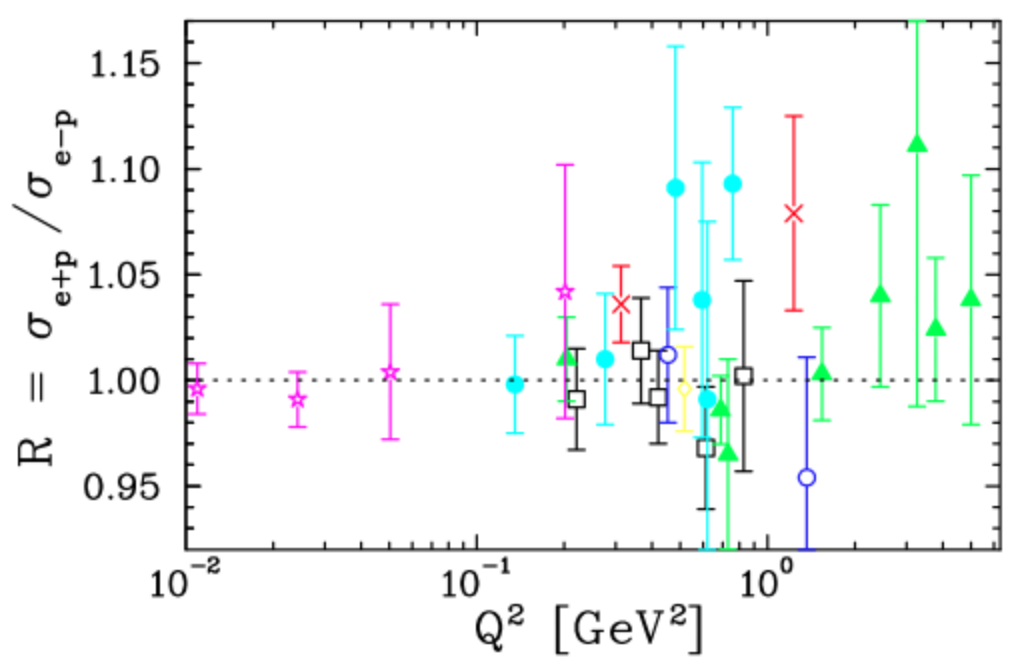

FIG. 19. The ratio of positron-proton to electron- proton elastic cross section, $R$ as a function of $Q^{2}$. The experiments are differentiated by color and symbol: black squares (Anderson et al., 1968), red crosses (Bouquet et al., 1968), green solid triangles (Mar et al., 1968), blue hollow circles (Bartel et al., 1967), yellow diamonds (Anderson et al., 1966), cyan filled circles (Browman et al., 1965) and magenta stars (Yount and Pine, 1962). The figure is adapted from Ref. [5].

\subsubsection{RECENT MEASUREMENTS AND ON-GOING WORK}

\section{Novosibirsk Experiment}

This experiment was carried out at the VEPP-3 storage ring in Novosibirsk, Russia with alternating positron and electron beams with energy $1.6 \mathrm{GeV}$ [30]. Elastic scattering events were detected simultaneously in three angular ranges with nonmagnetic detectors: small $\left(\theta \approx 10^{\circ}\right)$, middle $\left(\theta \approx 20^{\circ}\right)$ and large $\left(\theta \approx 65^{\circ}\right)$. At small angles, the TPE contribution is expected to be negligible and those data were used only for luminosity monitoring. The target was hydrogen gas stored in a openended cell. For the large angles where $\varepsilon=0.5$ and $Q^{2}=1.43 \mathrm{GeV}^{2}$, the result is $R=1.016 \pm 0.011 \pm 0.0003$ and for the middle angles where $\varepsilon=0.95$ and $Q^{2}=0.23$ $\mathrm{GeV}^{2}$, the result is $R=0.9976 \pm 0.009 \pm 0.0003$ where the first error is statistical and the second is systematic. Figure 21 shows these preliminary results compared with existing world data and hadronic calculations. These measurements are more precise 


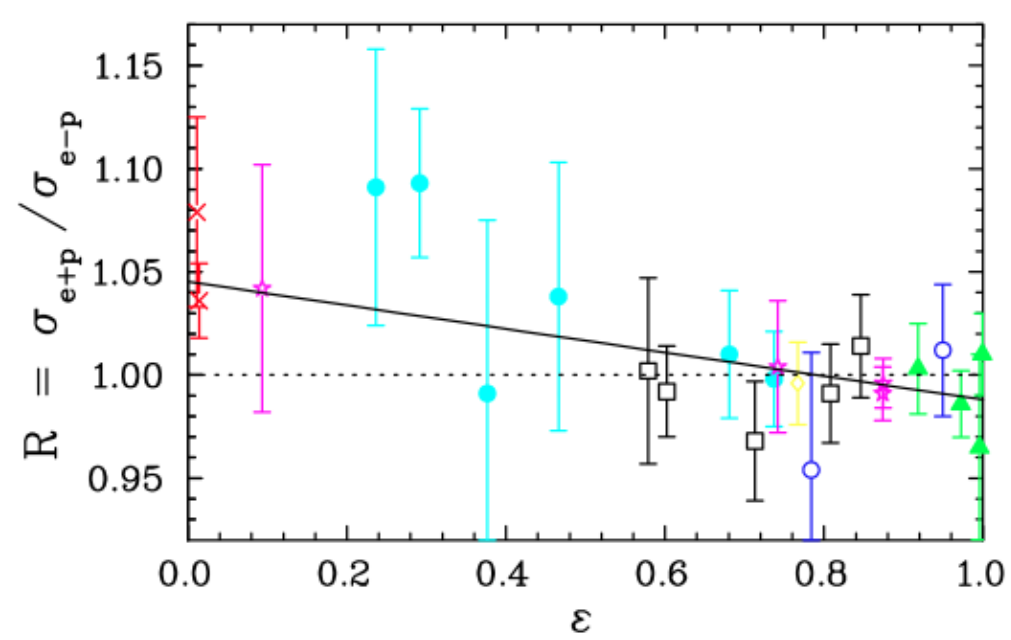

FIG. 20. The ratio of positron-proton to electron- proton elastic cross section, $R$ as a function of $\varepsilon$ for the measurements below $Q^{2}=2 \mathrm{GeV}^{2}$. The symbols are identical to Fig. 19. The solid line is the linear fit to the data assuming no $Q^{2}$ dependence and the slope is $-(5.7 \pm 1.8) \%$. The figure is adapted from Ref. [5].

than existing data and are consistent with the hadronic calculations by Blunden et al. [11].

\section{OLYMPUS Experiment}

The OLYMPUS experiment measured the elastic cross section ratios at the DORIS storage ring at DESY in Hamburg, Germany with alternating $2.3 \mathrm{GeV}$ positron and electron beams and a hydrogen internal gas target [31]. The beam was switched every morning to minimize the differences between electron and positron runs introduced by environmental factors. Data collection was completed in January 2013 and data analysis is in progress. The experiment is promised to measure the ratio $R$ in the range of kinematics, $0.6 \mathrm{GeV}^{2} \leq Q^{2} \leq 2.2 \mathrm{GeV}^{2}$ and $0.3 \leq \varepsilon \leq 0.9$ with $<1 \%$ uncertainty.

\section{CLAS TPE Experiment}

The CLAS TPE experiment [22] was carried out at Jefferson Laboratory in Hall B. The experiment presented a new technique for producing a mixed electron-positron 


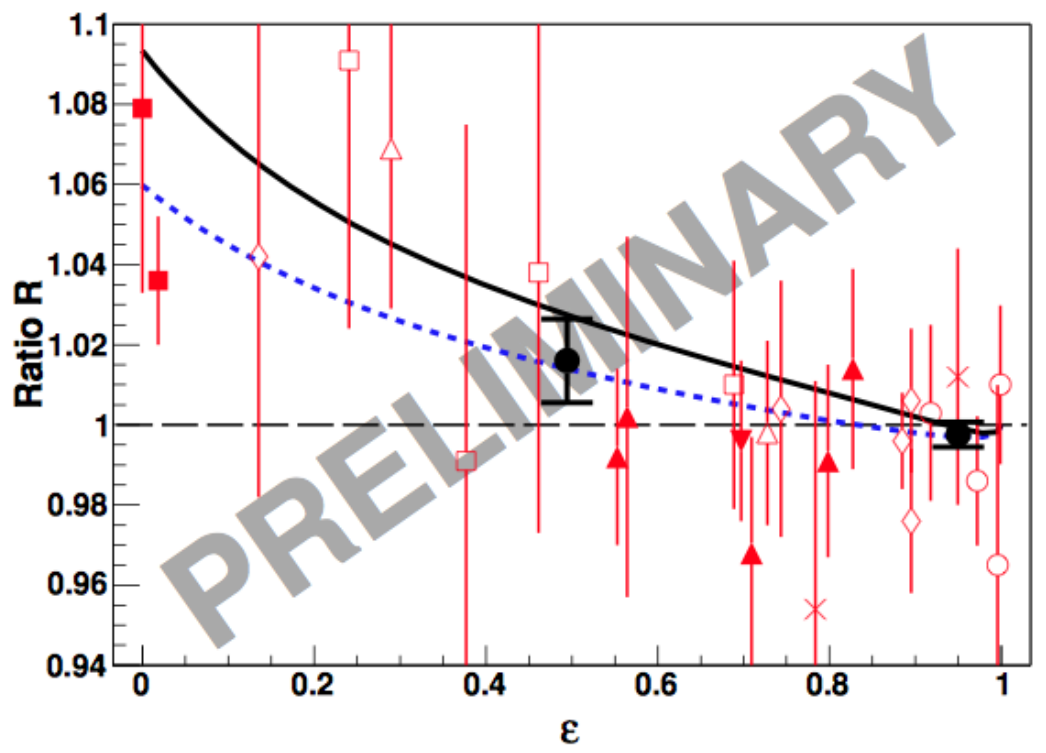

FIG. 21. Comparison of the preliminary results for the Ratio $R$ from the Novosibirsk experiment (black) with previous world data (red). The solid and dashed curves represent the theoretical calculations [11] for the ratio $R$ due to the two photon exchange correspond to the kinematics of run I and run II, respectively [30].

beam. The primary electron beam from the CEBAF accelerator was used to produce a secondary photon beam via bremsstrahlung and then those photons were converted into a tertiary positron/electron beam via pair-production. There was a test run performed in 2006 to demonstrate the capability of the new technique and the results were published. The mixed simultaneous positron/electron beams were scattered from a $18 \mathrm{~cm}$ liquid hydrogen target and the scattered events were detected by the nearly $4 \pi$ CLAS (CEBAF Large Acceptance Spectrometer) detector system. The results of the CLAS TPE test run was limited to large $\varepsilon$ and small $Q^{2}$ but showed better statistical accuracy than previous data at similar kinematics. Figure 22 shows the CLAS TPE test run results and the world data.

This thesis describes the full CLAS TPE experiment that was carried out during November 2010 - February 2011 with a $30 \mathrm{~cm}$ liquid hydrogen target. Experimental details, data analysis techniques and the results of the experiment will be discussed in detail in the remaining chapters. 


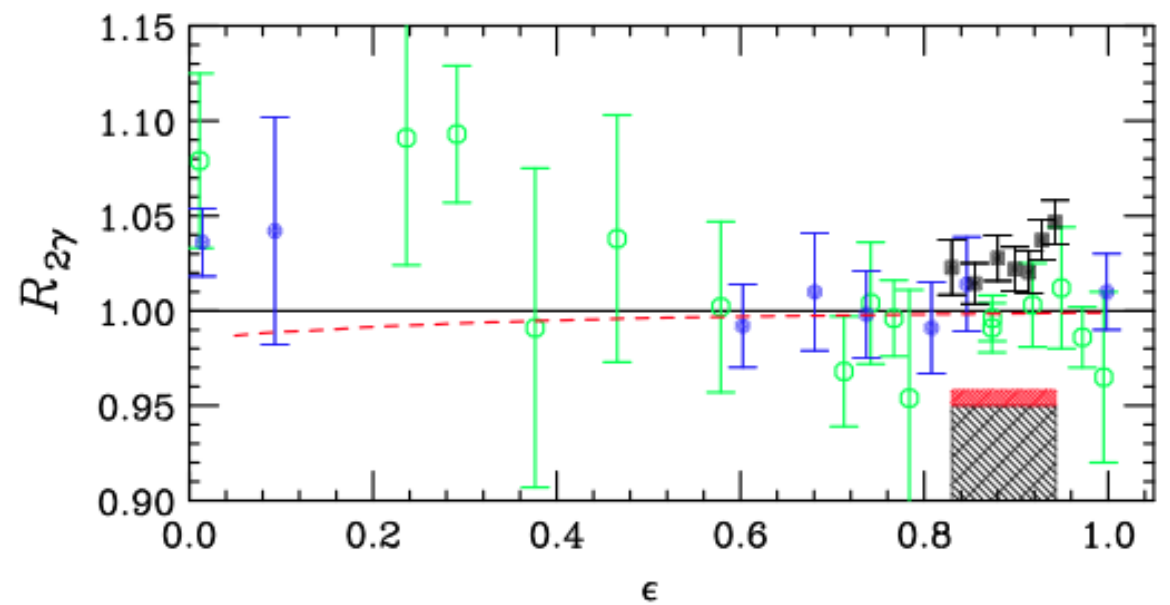

FIG. 22. A comparison of the CLAS TPE test run results (black filled squares) at $Q^{2} \approx 0.206 \mathrm{GeV}^{2}$ with previous world data at $Q^{2}<2 \mathrm{GeV}^{2}$ (green hollow circles). Blue filled circles represent the previous world data at similar $Q^{2}$ as this experiments and the red dashed curve is the hadronic calculation by Blunden et al. [11] The red shaded band and the black shaded band represent the point-to-point systematic uncertainty $(1 \sigma)$ and scale-type systematic uncertainty (due to relative luminosity) of the data [22]. 


\section{CHAPTER 3}

\section{TPE EXPERIMENT}

The objective of the TPE experiment is to measure the electron-proton and positron-proton elastic cross sections simultaneously to determine the cross section ratio $R=\sigma\left(e^{+} p\right) / \sigma\left(e^{-} p\right)$ for a wide range of $Q^{2}$ and $\varepsilon$. In this chapter we will discuss the technical details of the TPE experiment.

The TPE experiment took place in Hall B of the Thomas Jefferson National Accelerator Facility (TJNAF) using the primary electron beam provided by the Continuous Electron Beam Accelerator Facility (CEBAF). The $5.5 \mathrm{GeV}$ electron beam passed through a radiator where it produced bremstrahlung photons. The electrons were dumped using the tagger magnet. The photons were then passed through a converter where some of the photons converted into electron/positron pairs via pairproduction. In order to separate the leptons from the remaining photons, we passed the combined beam through a dipole magnet. It bent the lepton beams outward from the beamline so the photons could be stopped by a piece of tungsten (photon blocker) located on the beamline. We used two more dipole magnets to recombine the lepton beams. The recombined lepton beams were passed through several collimators, beam monitors and shielding and finally scattered from a liquid hydrogen target located at the center of the CEBAF Large Acceptance Spectrometer (CLAS). The scattered leptons and protons were detected in the various detectors of CLAS. Fig. 23 shows the layout of the beamline. The technique was tested in 2006 by the same collaboration and the details can be found in [22]. A full description of each component in the beamline and detector system and other important experimental details will be provided in this chapter.

\subsection{CEBAF ACCELERATOR}

The CEBAF accelerator consists of a polarized electron source, an injector, and a pair of superconducting RF linear accelerators (LINACs) connected to each other by two arc sections containing bending magnets. The electrons are emitted from the injector and accelerated through the two LINACs. Each LINAC includes 20 


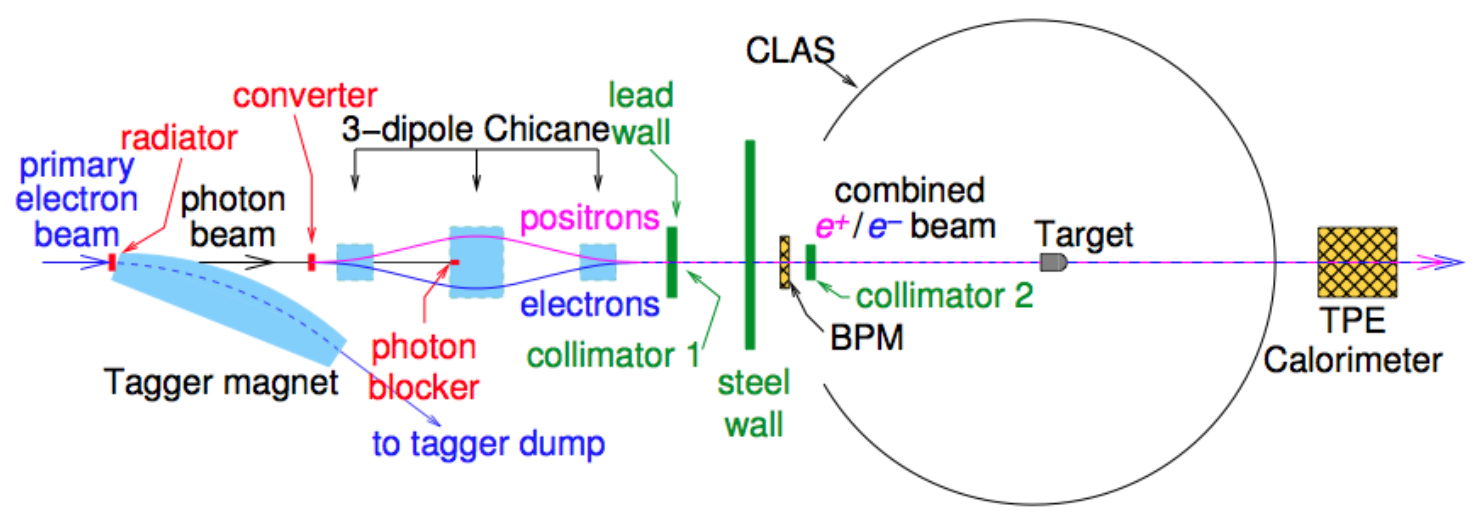

FIG. 23. Beamline sketch for the CLAS TPE experiment

cryomodules, and each module containing eight superconducting niobium cavities maintained at $2 \mathrm{~K}$ using liquid Helium. The electron beam can be circulated around the racetrack up to five times, gaining energy up to $\sim 600 \mathrm{MeV}$ with each pass through each LINAC. This produces electron beam with energies up to $6 \mathrm{GeV}$. On each pass, the electron beam can be separated and sent to three different experimental halls $\mathrm{A}, \mathrm{B}$, or $\mathrm{C}$, for simultaneous experiments.

\subsection{BEAMLINE COMPONENTS}

\subsubsection{RADIATOR}

The radiator is a very thin $\left(9 \times 10^{-3}\right.$ radiation lengths $)$ gold foil. The incoming $110-140 \mathrm{nA}, 5.5 \mathrm{GeV}$ electron beam passes through the radiator, scattering from the electric field of the gold nuclei and emitting photons by bremstrahlung radiation. Gold with its large atomic number and corresponding thin radiation length (6.4 $\mathrm{g} / \mathrm{cm}^{2}$ ) reduces the electron energy loss and multiple scattering for a given thickness in radiation lengths. During the experiment we optimized the radiator thickness to maximize the intensity of the $e^{+} / e^{-}$beam.

\subsubsection{TAGGER MAGNET}

The recoil electrons and bremstrahlung photons then pass through a dipole magnet called the "tagger magnet". The magnetic field of the tagger directs electron 


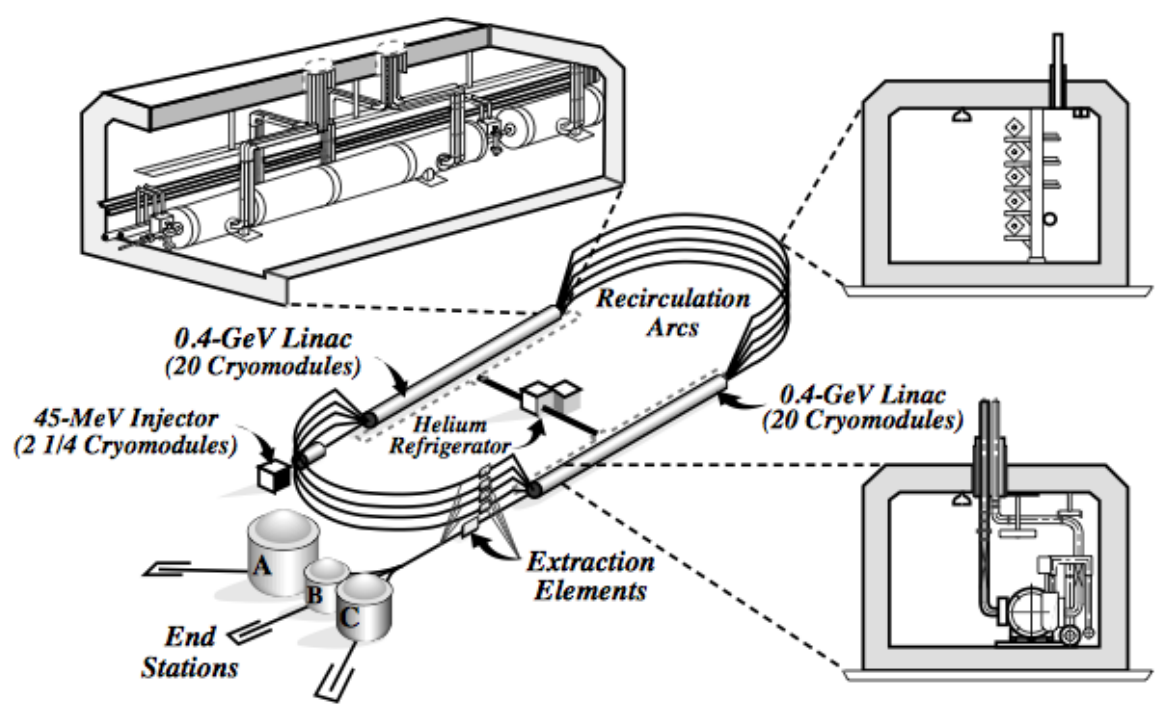

FIG. 24. A schematic layout of the CEBAF accelerator [32].

which did not radiate to the underground beam dump. Electrons which did radiate bend more and hit the hall floor inside of a large shielding hut. The scintillator counters in the photon tagger were also removed before the TPE experiment to prevent exposure to high radiation and were reinstalled after the TPE run was completed.

\subsubsection{CONVERTER}

The converter is also a very thin $\left(9 \times 10^{-2}\right.$ radiation lengths $)$ foil. It produces electrons and positrons from the incoming photon beam through the pair production process. During the experiment we optimized the thickness of the converter to minimize the background rate to maximize the luminosity.

\subsubsection{CHICANE}

The chicane consists of three dipole magnets. The first and third dipole magnets are called "Italian dipoles" and the second dipole is called the pair spectrometer magnet. The Italian dipoles are $0.5 \mathrm{~m}$ long and the pair spectrometer is $1 \mathrm{~m}$ long. For the experiment, the Italian dipoles and pair spectrometer were operated at magnetic fields of $B \simeq \pm 0.49 \mathrm{~T}$ and $B \simeq \mp 0.44 \mathrm{~T}$, respectively. When the combined leptons and photons passed through the first Italian dipole, the lepton beams bent outward 
from the beamline so that the photons could be stopped by a $4 \mathrm{~cm}$ wide $35 \mathrm{~cm}$ long tungsten photon blocker located on the beamline. The other two dipole magnets were used to recombined the lepton beams.

All three dipoles were left-right symmetric and therefore the lepton beams should have been identical. The final beam energy of the leptons was $\sim 0.5-5.4 \mathrm{GeV}$. The lepton momentum acceptance was limited by the width of the photon blocker $(4 \mathrm{~cm})$ and aperture of the pair spectrometer magnet $(40 \mathrm{~cm})$.

The magnetic fields of the three dipoles were optimized using the beam position profiles measured from the sparse fiber monitor (SFM) and more details will be discussed in Section 3.3.1. In order to study the lepton beams individually, we used two lead slabs (beam blockers). The beam blockers were inserted at the entrance of the pair spectrometer for such diagnostic measurements.

In order to reduce the background produced by the chicane, we built two large shielding structures, one between the first and second magnets and one between the second and third magnets. Figure 25 shows the layout of the chicane magnet, shielding and photon blocker obtained from GEANT4 simulations. The chicane shielding structures include layers of concrete, lead and borated polyethylene. The magnetic fields of the chicane magnets were periodically reversed during the TPE run to reduce systematic uncertainties.

\subsubsection{TARGET}

The TPE target is a $30 \mathrm{~cm}$ long, $6 \mathrm{~cm}$ diameter, $0.127 \mathrm{~mm}$ thick kapton cell filled with liquid hydrogen. Due to the beam divergence, the mixed lepton beams were relatively large at the target and therefore a larger diameter target was necessary. The target was placed along the beamline near the center of the CLAS. The liquid hydrogen was cooled using a cryogenic system. There was a copper heat exchanger attached to the target which was cooled by liquid hydrogen while liquifying the hydrogen. An engineering drawing and a photograph of the TPE target is shown in Fig. 26.

\subsubsection{OTHER BEAM LINE COMPONENTS}

In addition to the main beamline components mentioned above, we used three collimators to reduce background. The main purpose of these collimators was to limit and define the direction and divergence of the beam. The first collimator, called the 


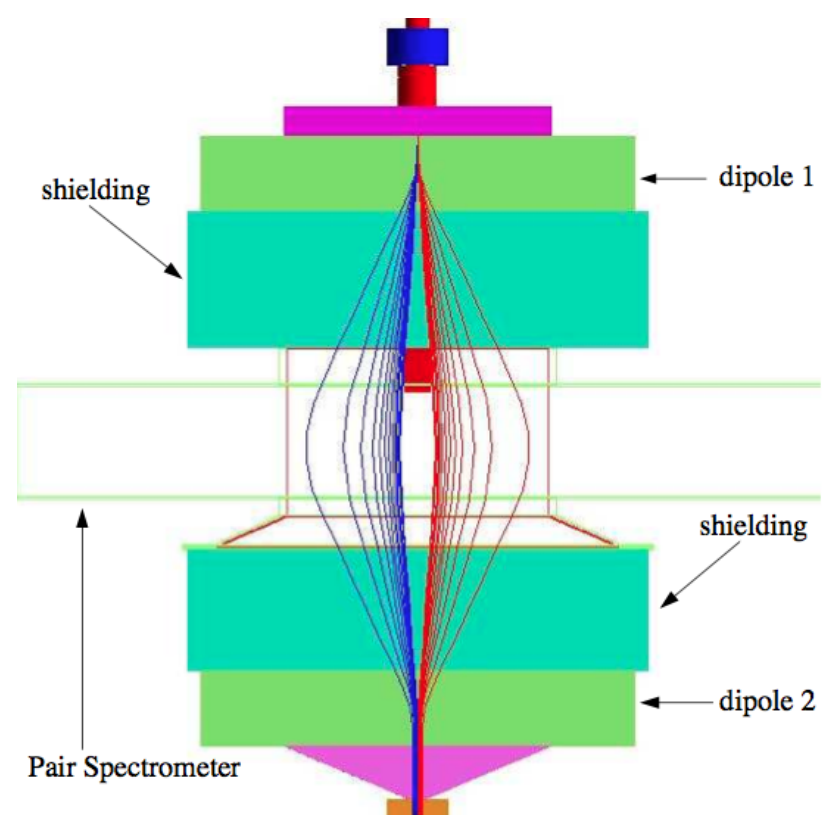

FIG. 25. The simulated trajectories of the leptons through the chicane magnetic system [33]. The electron and positron trajectories are shown in red and blue, respectively. The beam enters from the top and travels downwards.

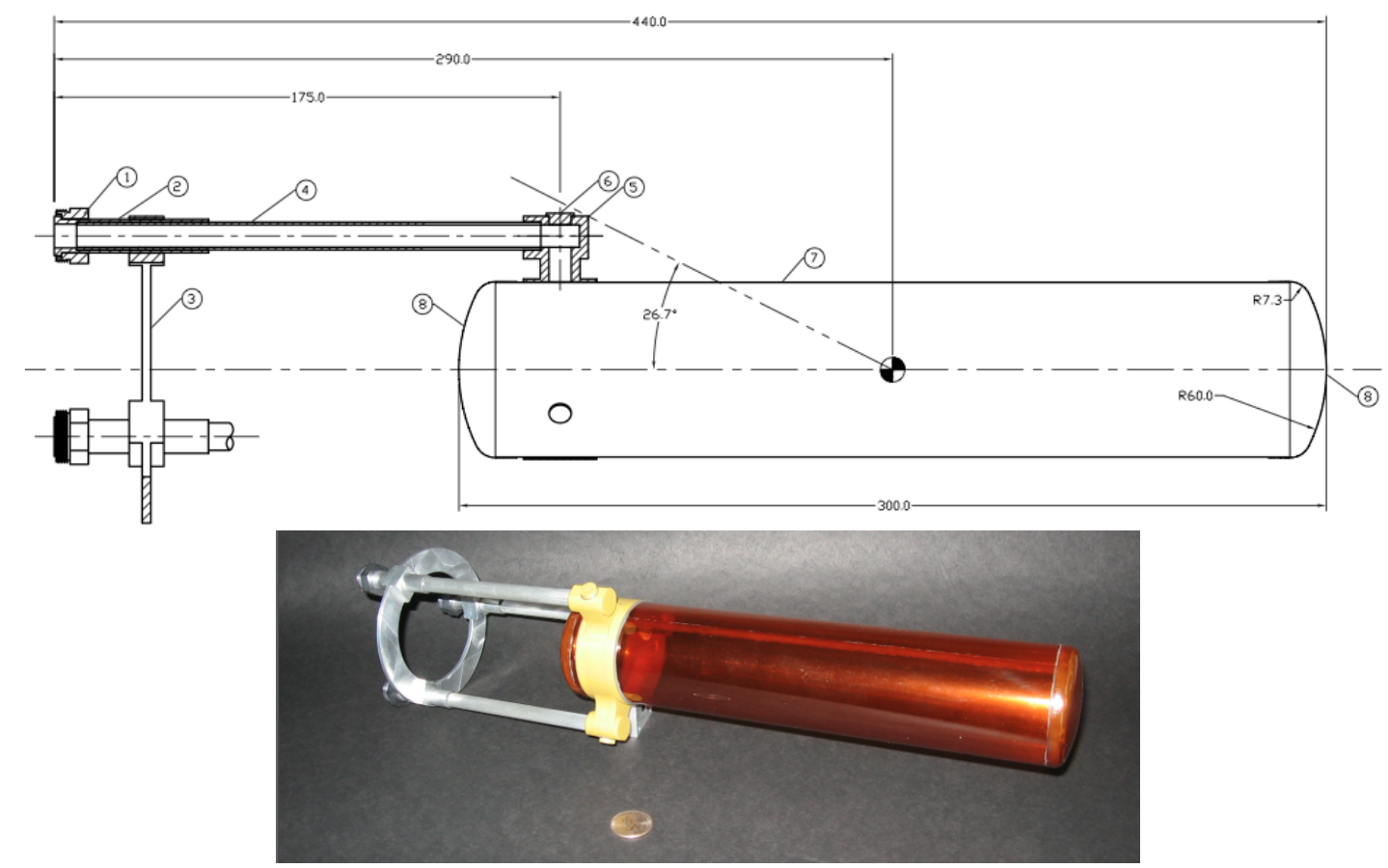

FIG. 26. An engineering drawing, with dimensions in mm (top) and a photograph of the TPE liquid hydrogen target. 
"photon collimator", was a $12.7 \mathrm{~mm}$ inner diameter, $30 \mathrm{~cm}$ long, nickel collimator. It was installed between the tagger magnet and the converter. It significantly reduced the background rates at the CLAS TOF counters. The second collimator was a $1.75 \mathrm{~cm}$ inner diameter, tungsten collimator located after the third chicane dipole. The third collimator was a $4 \mathrm{~cm}$ inner diameter lead collimator installed just a few meters upstream of the target. The combination of these three collimators resulted in a combined lepton beam of about $6 \mathrm{~cm}$ diameter incident on the target.

\subsubsection{SHIELDING}

The backgrounds caused by the beamline components were extensively studied before and after the TPE test run in 2006. A GEANT3 simulation toolkit was initially used. It was later updated to GEANT4 because of the more precise physics and secondary interaction packages. More details of the GEANT4 simulations built for the TPE experiment can be found at [33, 34]. The photon collimator and the photon blocker were identified as the main sources of low energy photon and neutron backgrounds in the TOF, respectively. There was also a low energy electron background originating at the entrance and exit of the chicane dipole magnets and the beam pipe. In order to reduce these backgrounds, numerous shielding were designed and simulated.

There were two large shielding structures between the two Italian dipoles and pair spectrometer built with concrete, lead and borated polyethylene. The photon collimator was shielded with lead and concrete. There was also a $1 \mathrm{~m} \times 1 \mathrm{~m} \times 0.1$ $\mathrm{m}$ thick lead wall installed immediately after the chicane and a $4 \mathrm{~m} \times 4 \mathrm{~m}$ steel wall placed approximately $2 \mathrm{~m}$ upstream of the entrance to CLAS. Additional shielding was placed on the floor around the tagger dump area. In order to reduce radiation, the sensitive electronics located along the beamline were shielded with lead walls, concrete walls and lead blankets (40 lbs, 18 inch $\times 22$ inch). Figure 27 shows some of the shielding used in TPE experiment.

The size (diameter, thickness, width, length) and material of all beamline components were initially optimized by the GEANT4 simulation. During the commissioning phase of the experiment the radiator thickness, convertor thickness, photon collimator inner diameter, and second collimator inner diameter were optimized to maximize luminosity while keeping the drift chamber occupancies below 3\%. Important parameters of the beamline components during the TPE run period are summarized in 

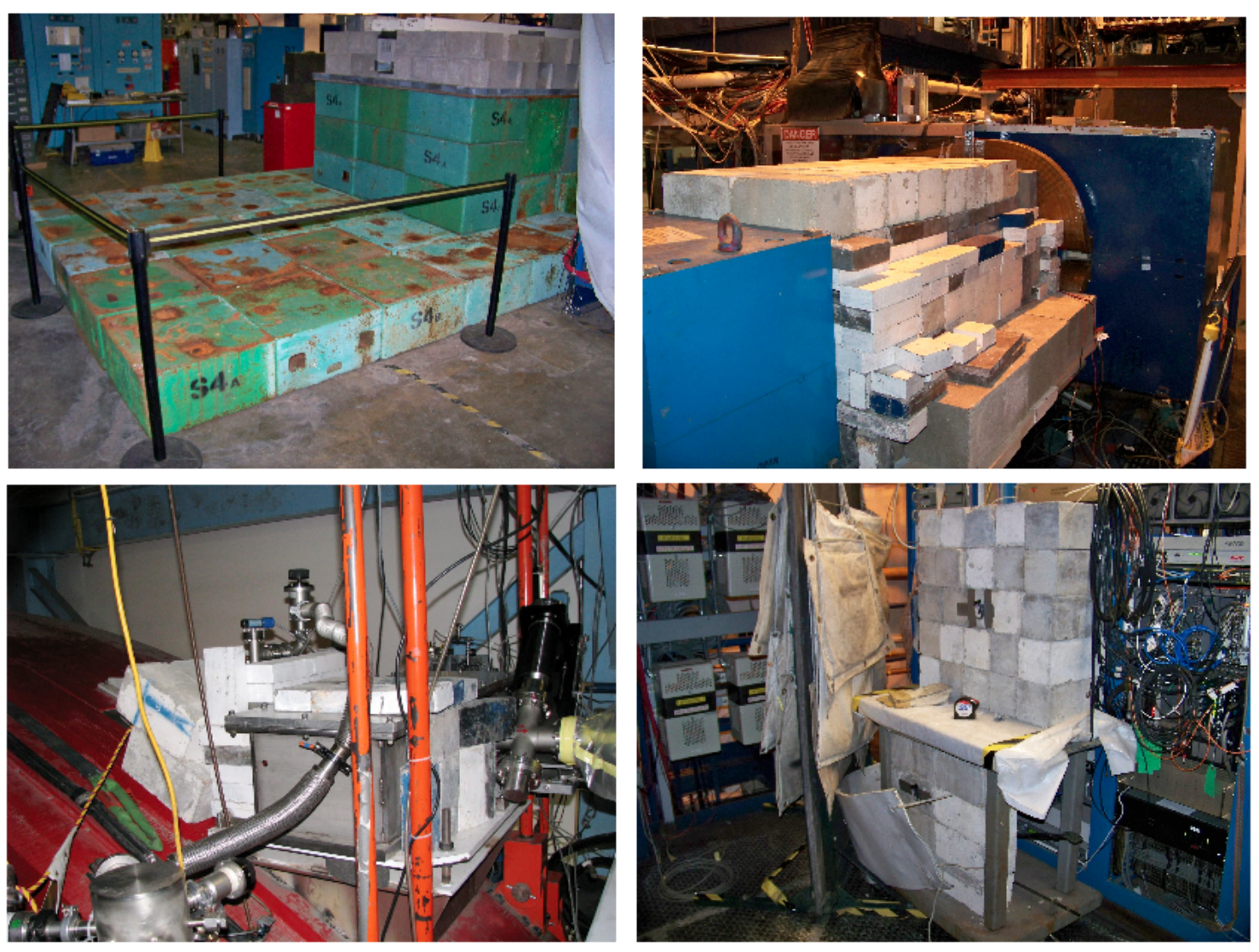

FIG. 27. Photographs of the shielding taken during engineering work, top-left: tagger bunker shield, top-right: Shielding between pair spectrometer and second Italian dipole, bottom-left: photon collimator shield, bottom-right: lead blankets and concrete shielding on electronics. 
Table 1.

\begin{tabular}{|c|c|}
\hline Primary beam & $\begin{array}{c}110 \leq I \leq 140 \mathrm{nA} \\
E=5.5 \mathrm{GeV}\end{array}$ \\
\hline Radiator (gold) & $9 \times 10^{-3} \mathrm{RL}$ \\
\hline Photon collimator & $12.7 \mathrm{~mm} \mathrm{ID}$ \\
& $9 \times 10^{-2} \mathrm{RL}$ \\
\hline Converter (gold) & $B \approx 0.49 \mathrm{~T}$ \\
& $L \approx 0.5 \mathrm{~m}$ \\
\hline Italian dipole & $B \approx 0.44 \mathrm{~T}$ \\
& $L \approx 1 \mathrm{~m}$ \\
\hline Pair spectrometer & $1.75 \mathrm{~cm} \mathrm{ID}$ \\
\hline Lepton collimator 1 (tungsten) & $4 \mathrm{~cm} \mathrm{ID}$ \\
\hline Lepton collimator 2 (lead) & diameter $=6 \mathrm{~cm}$ \\
\hline LH2 target & $\pm 1500 \mathrm{~A}$ \\
\hline CLAS torus current & $4000 \mathrm{~A}$ \\
\hline Mini-torus current & $\mathrm{cm}$ \\
\hline
\end{tabular}

TABLE 1. TPE Run conditions. ID = Inner Diameter, $\mathrm{RL}=$ radiation lengths.

\subsection{BEAM MONITORS}

\subsubsection{SPARSE FIBER MONITOR (SFM)}

In order to minimize systematic uncertainties, the two lepton beams should each be stable and centered on the beamline. The SFM is located just upstream of the target and designed to monitor the position and spread of the lepton beams. It was built and tested at Florida International University. Its $6 \times 6$ inch aluminum frame supports 32 scintillating fibers. The fibers are $1 \times 1 \mathrm{~mm}$ multiclad Bicron (BCF-12) fibers. Two sets of 16 fibers each were positioned vertically and horizontally. Each set was glued to a Hamamatsu multi anode PMT that amplifies and transfers the 
signals gathered by the fibers to readout electronics. The schematic diagram of the monitor is shown in Fig. 28. A typical beam distribution measured by the SFM is shown in Fig. 29.

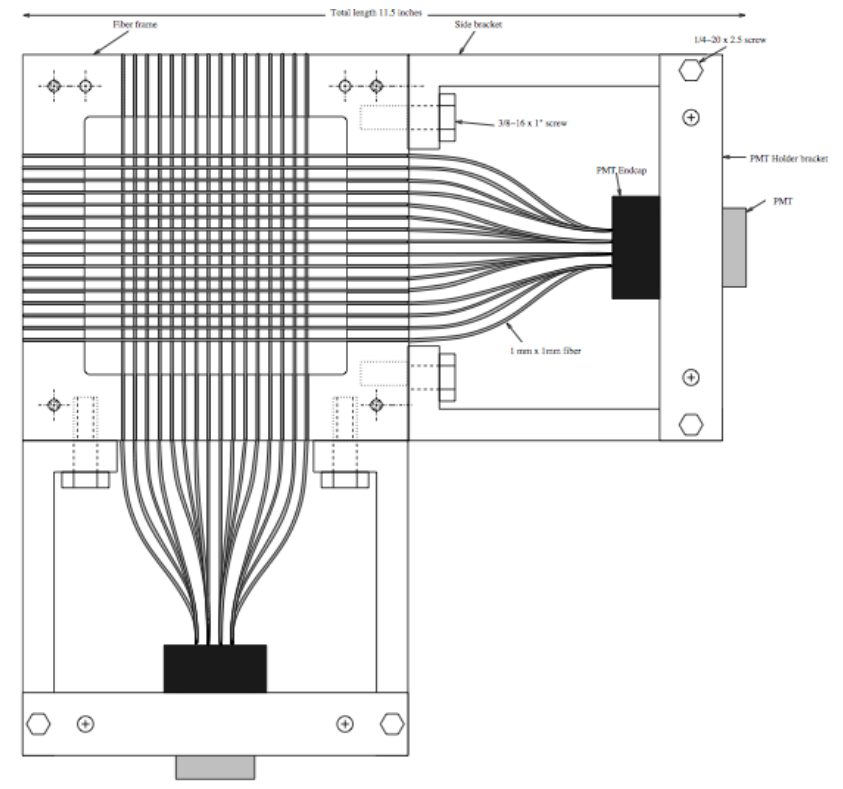

FIG. 28. The TPE sparse fiber monitor.

During the commissioning of the experiment and after each chicane polarity reversal (flip), the beam center for the individual lepton beams was measured and plotted for various chicane (Italian dipole) currents (see Fig. 30). The intersection of the center position of lepton beams in Fig. 30 was used to optimize the chicane current. The SFM was used continuously during the experiment to monitor the lepton beam position and its stability.

\subsubsection{DENSE FIBER MONITOR (DFM)}

The DFM was used in conjunction with the SFM to determine the parallelism of the incident electron and positron beams. It consists of two arrays of fibers $(64 \times 64)$ arranged in horizontal and vertical planes, with $2 \mathrm{~mm}$ diameter optical fibers. The DFM was mounted on the TPE calorimeter and therefore was only positioned in the beamline during the special low luminosity runs. 

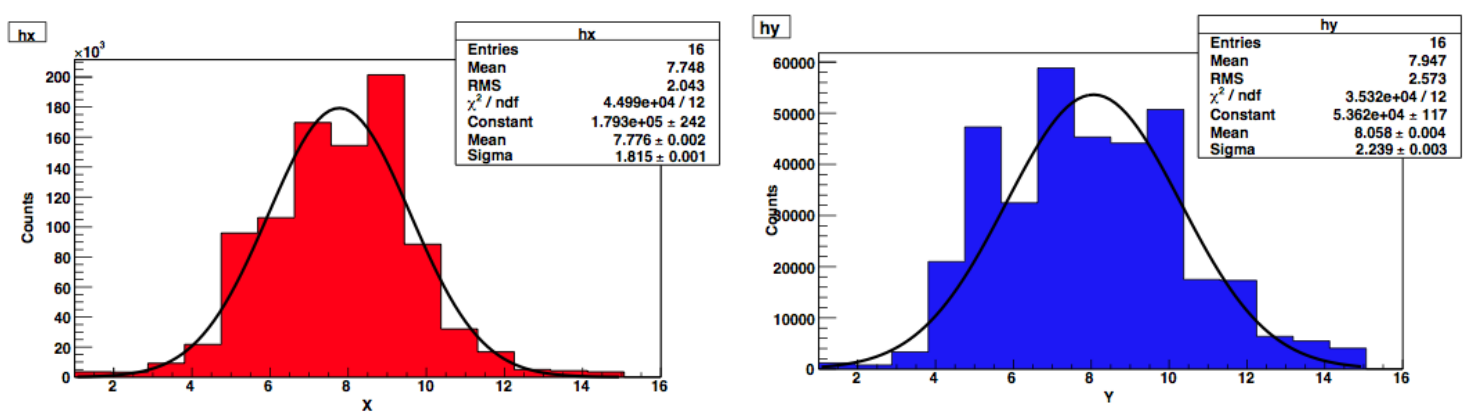

FIG. 29. The $x$-horizontal (left) and $y$-vertical (right) beam position distributions measured by the sparse fiber monitor.
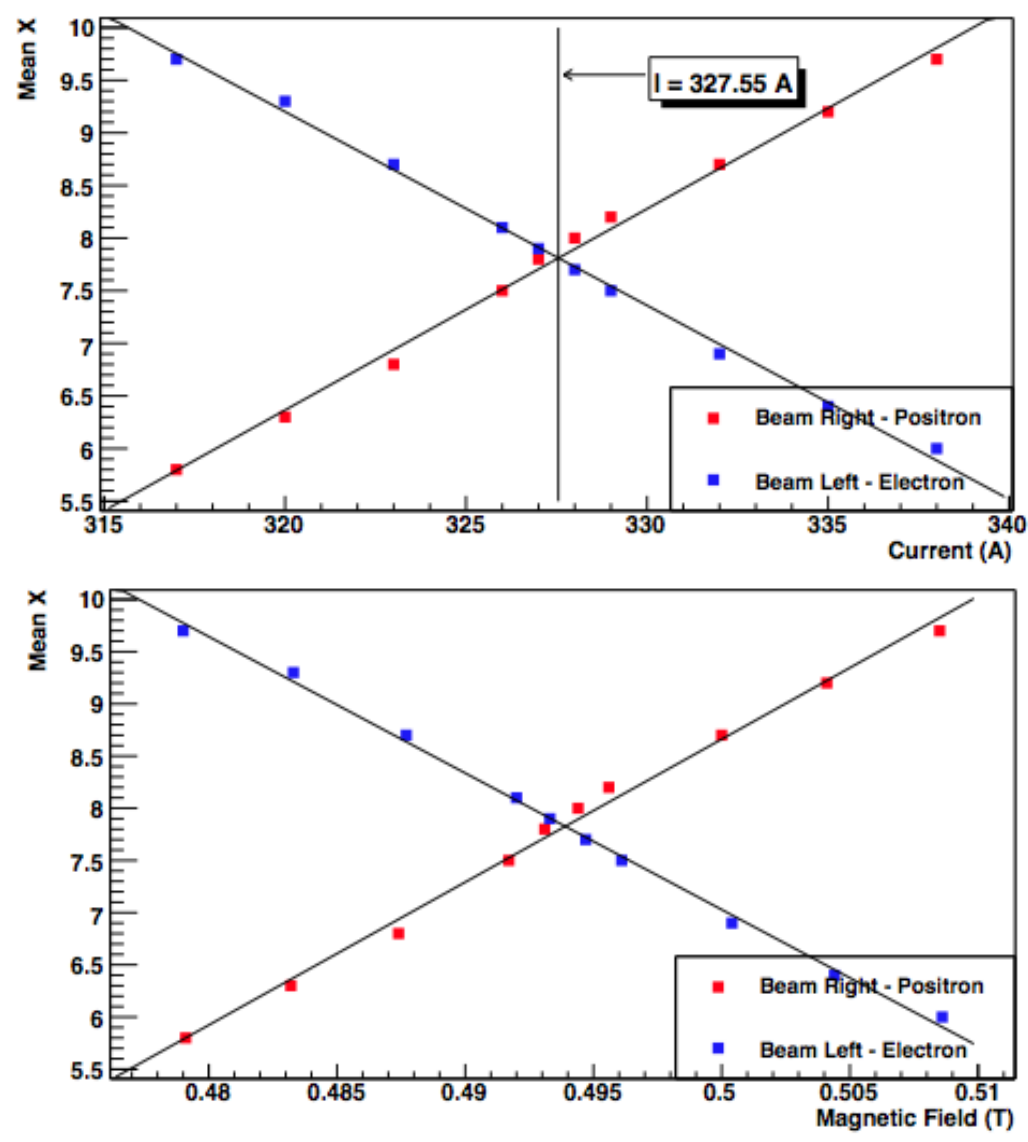

FIG. 30. Individual positron and electron beam position centroids as a function of Italian Dipole current (top) and magnetic field (bottom) as measured by the sparse fiber monitor. The positron beam position was measured with the electron beam blocked and vice versa. 

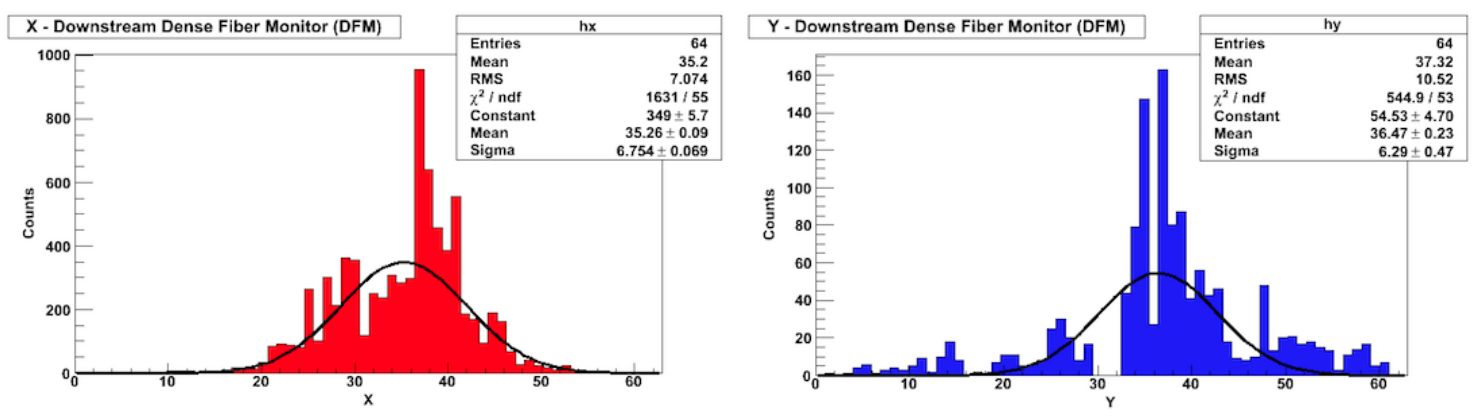

FIG. 31. The hit distribution for the left beam passing through the chicane as measured by the dense fiber monitor. The $x$ and $y$ positions are in fiber number.

Figure 31 shows the EPICS (for beam position monitoring online) plot of DFM readout taken during a TPE calorimeter run. The efficiency varies from channel to channel. Hence the DFM was unable to make a reliable measurement of absolute position. However, it did show that both the electron and the positron beams had the same centroid and that therefore both beams were coincident and parallel.

\subsubsection{TPE CALORIMETER}

The TPE electromagnetic calorimeter (TPECal) was installed in the beamline to measure the energy distributions of the individual lepton beams. The TPECal consists of 30 shashlik modules arranged in five rows of six modules (see Fig. 32). The shashlik modules used in the TPE experiment were $3.82 \times 3.82 \times 45 \mathrm{~cm}^{3}$ and consisted of alternating layers of $1 \mathrm{~mm}$ lead and $2 \mathrm{~mm}$ scintillant. There were 16 wavelength shifting fibers (1.5 mm diameter spaced $7.7 \mathrm{~mm}$ apart) in each module to transmit the light from the individual scintillator layers to the $25 \mathrm{~mm}$ diameter Hamamatsu R3998-02 photomultiplier tubes (PMTs). The shashlik modules, PMTs and bases of the calorimeter were provided by the nuclear physics group at the Universidad Tecnica Federico Santa Maria in Valparaiso, Chile and were assembled at Old Dominion University (ODU). The box and support structure were designed and machined at ODU. The assembled TpeCal was mounted on the forward carriage downstream of the CLAS detector with the ends of the shashlik modules pointed at the target (upstream) and the PMTs pointed away from the target (downstream). The dense fiber monitor (DFM) was mounted on the upstream face of the TPECal. 

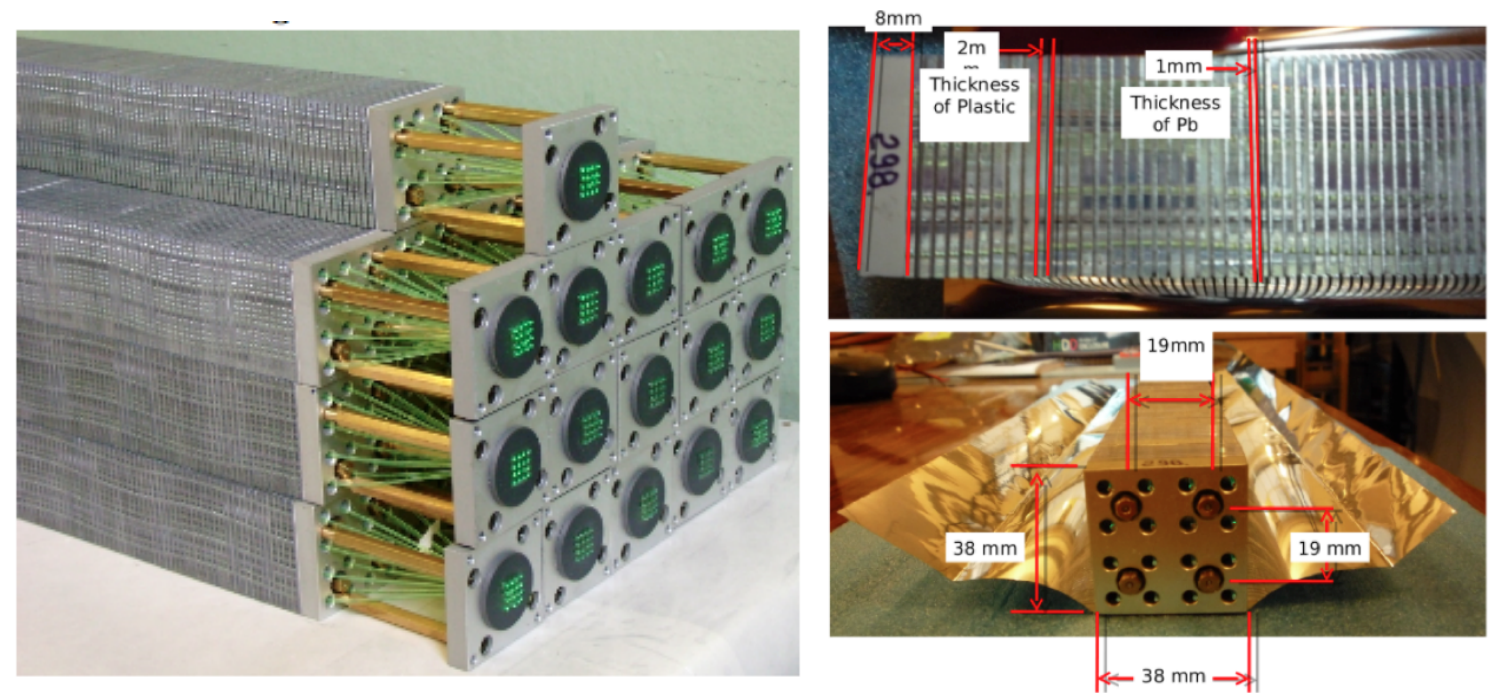

FIG. 32. A photograph of a TPE calorimeter taken during the assembly process.

The TPECal/DFM was used only for low luminosity runs before and after each chicane flip. It was moved horizontally into and out of the beam. In order to calibrate the TPECal, cosmic ray data was also taken after the completion of the experiment with the calorimeter positioned vertically. ADC pedestals and relative gains of each calorimeter module were determined by those cosmic data.

\subsection{HALL B AND CLAS DETECTOR}

Hall B has the nearly $4 \pi$ CEBAF Large Acceptance Spectrometer (CLAS) detector system. CLAS consists of six superconducting coils which produce a toroidal magnetic field around the beam axis and divide the detector into six sectors. Each sector has three regions of drift chambers [35] to determine the trajectories of charged particles, Cherenkov counters [36] for electron identification, scintillation counters [37] for measuring time-of-flight (TOF) and electromagnetic calorimeters [38] to detect showering particles (electrons and photons) and neutrons. Figure 33 shows views of the CLAS detector. The TPE experiment did not use the Cherenkov counters. Each of those detectors will be discussed in more detail in the remaining sections. We also used the mini-torus magnet which was placed close to the target to prevent the low momentum electrons produced by Moller scattering in the target from reaching the drift chambers. 

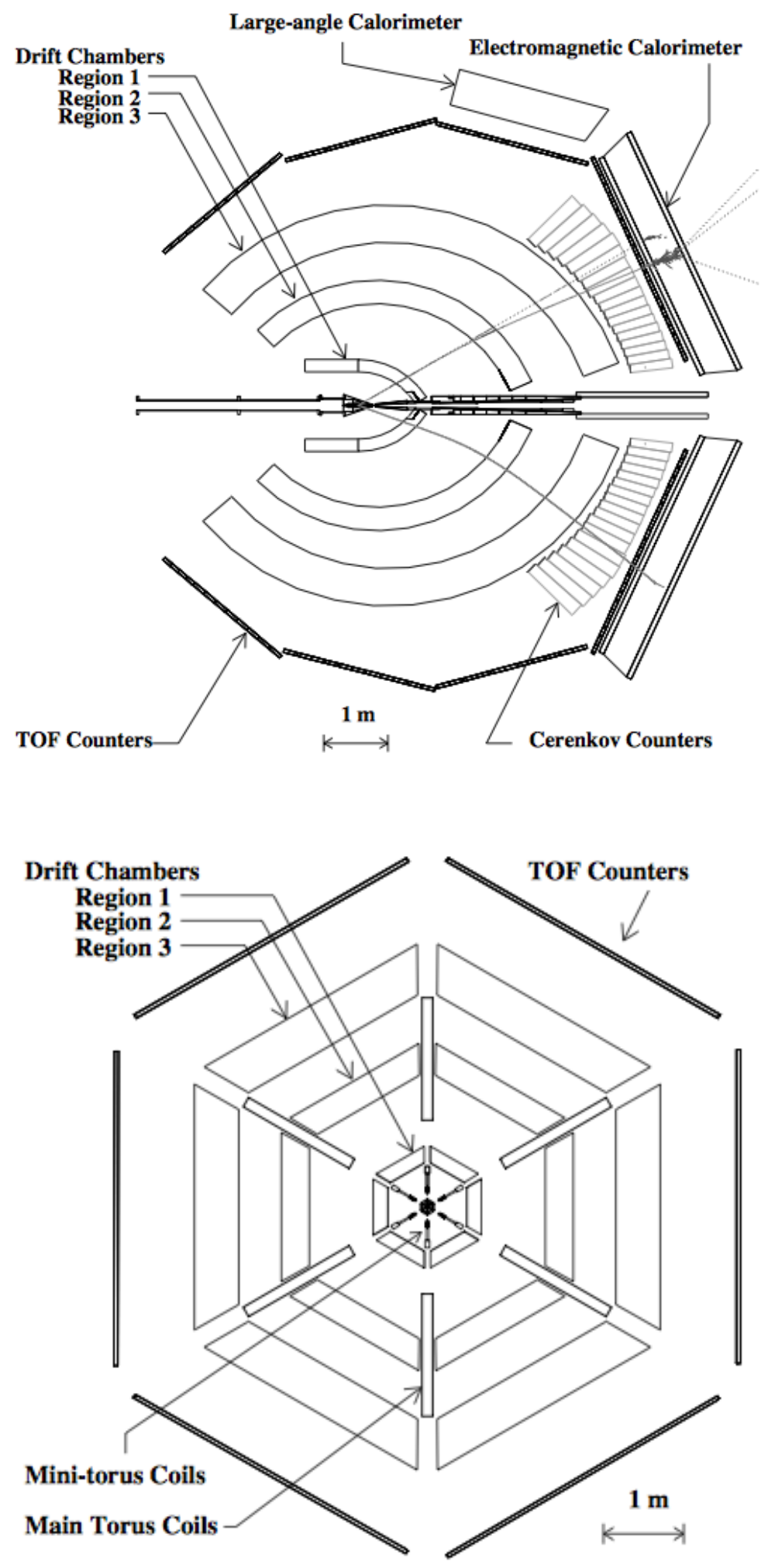

FIG. 33. A schematic layout of the CLAS detector cut along the beam line (top) and cut perpendicular to beam (bottom) [32]. Typical electron, proton and photon tracks from an interaction point are also shown in top figure. 


\subsection{DETECTOR SYSTEM}

This section describes each component of the CLAS detector used for the TPE experiment. These components include drift chambers, TOF counters, the electromagnetic calorimeter, torus magnet and the mini-torus magnet.

\subsubsection{TORUS MAGNET}

The CLAS torus magnet is composed of six kidney-shaped super conducting coils arranged radially around the beamline. The coils were designed, constructed and assembled by Oxford Instruments. It can produce a toroidal magnetic field with a peak value of $3.5 \mathrm{~T}$ at an operating current of $3860 \mathrm{~A}$, but is typically operating at $87 \%$ (3375 A) of the maximum current or less to keep internal mechanical stresses within conservative limits [32]. Each superconducting coil consists of four layers and each layer has 54 turns of aluminum-stabilized $\mathrm{NbTi} / \mathrm{Cu}$ conductor. The coils are cooled to a temperature of $4.5 \mathrm{~K}$ using liquid helium through cooling tubes located at the edge of the windings. The magnet is approximately $5 \mathrm{~m}$ in length and $5 \mathrm{~m}$ in diameter. The kidney shape of the magnet was designed to provide a strong magnetic field for the higher-momentum forward-going particles and a lower magnetic field for particles emitted at larger angles. A layout of the superconducting coils and their magnetic fields are shown in Fig. 34 .

For the TPE experiment, the torus magnet was operated at $1500 \mathrm{~A}$. The current was periodically reversed during the TPE run. When the torus magnet was operated at positive magnetic field, negatively-charged tracks were bent towards the beamline, and positively-charged tracks bent away the from the beamline. At negative torus magnetic field the bending reversed. By exchanging the paths of electrons and positrons through the CLAS, we could minimize the systematic uncertainty of the TPE measurements.

\subsubsection{MINI-TORUS}

In addition to the main torus magnet, there is a small normal conducting "minitorus" magnet located around the target inside of R1 drift chamber. It is typically operated during standard electron-beam operation and its integral magnetic field is about $5 \%$ of that of the main torus magnet. The main purpose of the mini-torus is to reduce the background produced by scattered Moller electrons. TPE test run 


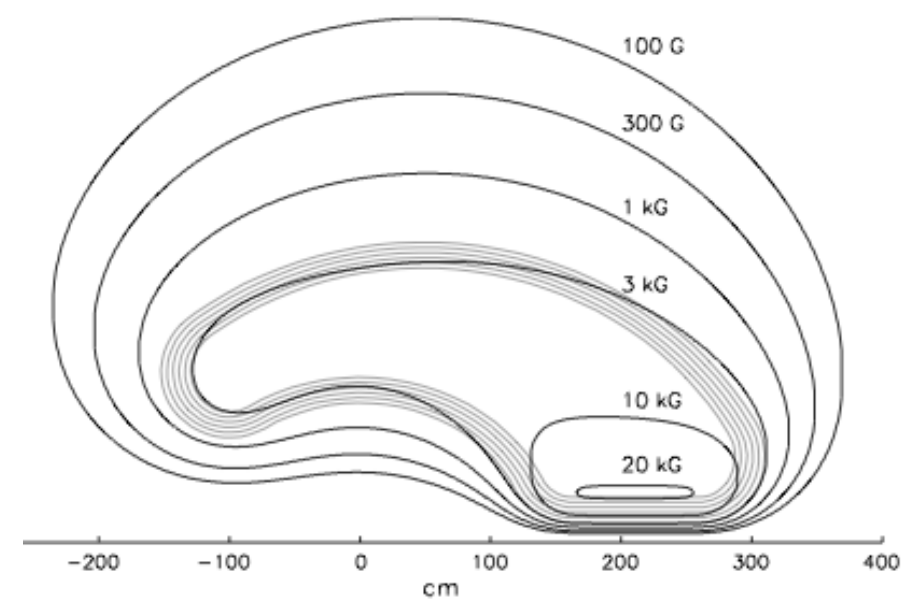

FIG. 34. Contours of constant absolute magnetic field for the CLAS toroid in the midplane between two coils [32].

data and simulations showed that without the mini-torus the low energy charged particle tracks passing through the Region 1 drift chamber got reflected by the torus magnetic field and traveled back through the Region 1 drift chamber giving multiple hits from the same track. In order to reduce this background, the mini-torus magnet was used with additional mass shielding. The mass shielding was made of tungsten, lead and steel (see Fig. 35). The mini-torus magnet was operated at $4000 \mathrm{~A}$ during the TPE experiment.

\subsubsection{DRIFT CHAMBER}

The trajectories of the charged particles are measured by the wire chamber system of CLAS known as Drift Chambers (DC) [35]. The DC consist of three regions (R1, R2, R3) each consisting six identical wedge-shaped sectors (see Fig. 36). Each region contains two superlayers. The first superlayer (axial) has the wires approximately parallel to the magnetic field lines and the second superlayer (stereo) has its wires at an angle of $6^{\circ}$ with them. Each superlayer contains six layers of sense wires except R1 stereo which has only four layers. Each sense wire is surrounded by six shared field wires to form a hexagonal pattern as shown in Fig. 37. To maintain a homogeneous electric field, each superlayer is surrounded by a layer of guard wires. The sense wires are $20.1 \mu \mathrm{m}$ gold-plated tungsten and the field wires are $140 \mu \mathrm{m}$ gold-plated 


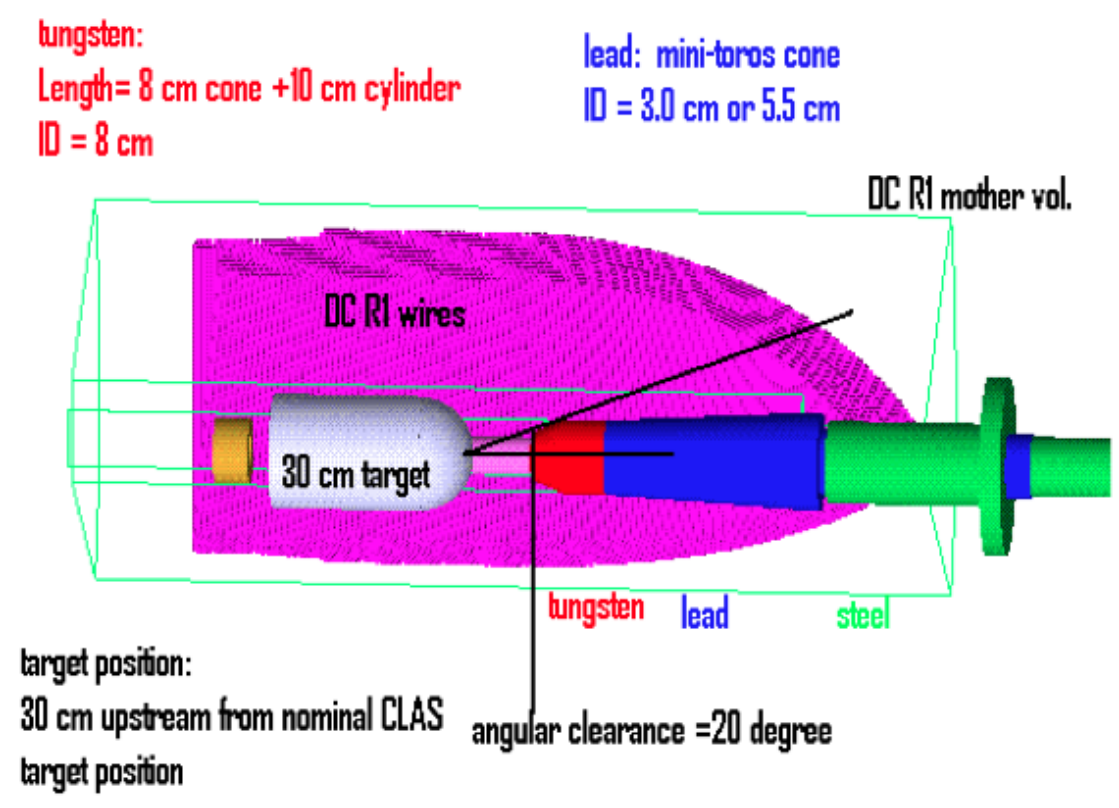

FIG. 35. GEANT4 simulation of the target area with the DC R1 mother volume, target and mass shielding [33]. The mini-torus (not shown) is located between the target and the Region 1 drift chamber.

aluminum.

The chambers are filled with a gas mixture of $90 \%$ Argon and 10\% carbon dioxide which provides good efficiency, adequate resolution and reasonable collection times. The field and sense wires are kept at negative and positive high voltages, respectively to create a potential difference between them. Electrons freed by the charged tracks ionize the gas molecules as they travel towards the sense wires. These electrons are registered as an electric pulse, amplified and recorded by TDCs (time-to-digital converters). The R2 drift chambers are mounted on the torus magnet and therefore operate in a magnetic field up to $2 \mathrm{~T}$.

The drift chambers cover the polar angle $8^{\circ}<\theta<142^{\circ}$ and are designed to determine the trajectory of charged particles of momentum above $200 \mathrm{MeV} / \mathrm{c}$ with polar angle resolution of $1 \mathrm{mrad}$ and momentum resolution of $0.5 \%$.

\subsubsection{TOF COUNTER}

The TOF counters [37] cover the polar angle between $8^{\circ}$ to $142^{\circ}$ and the entire 

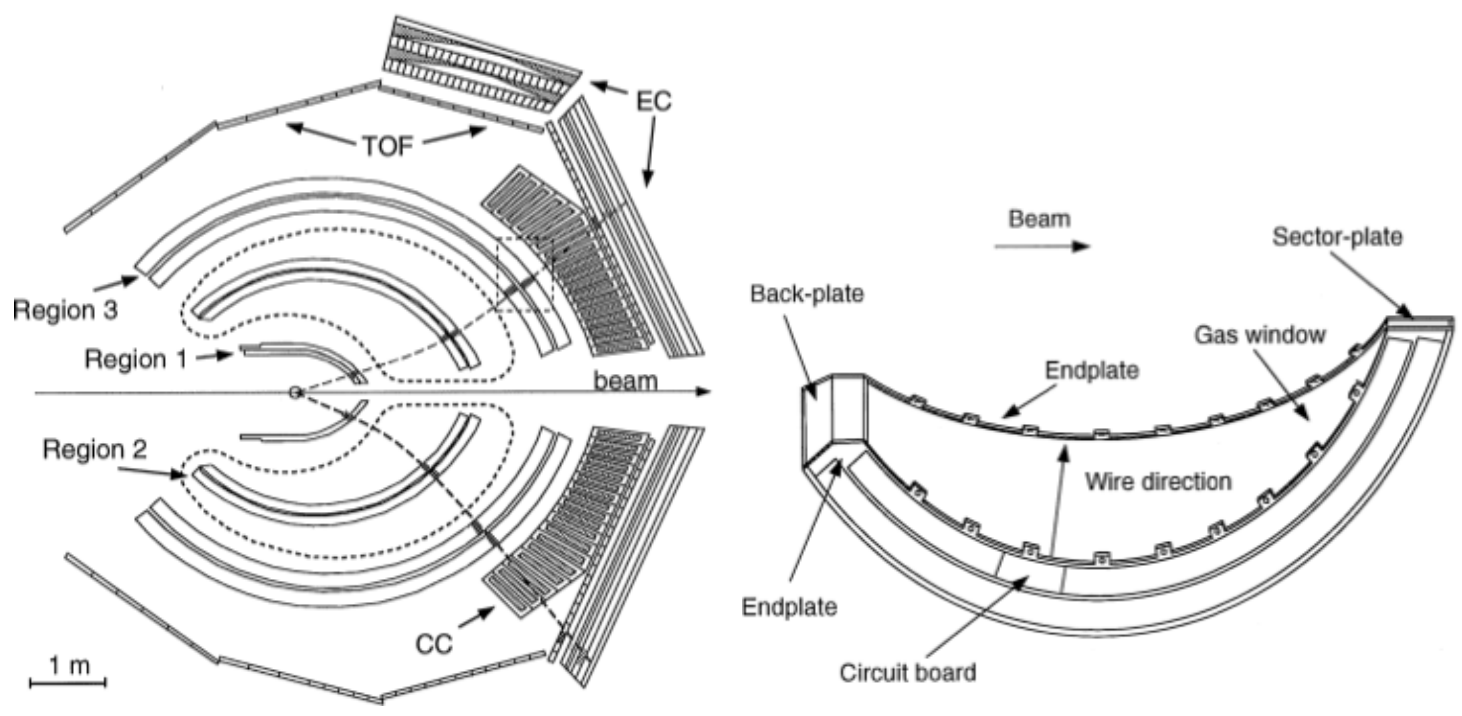

FIG. 36. Horizontal cut through the CLAS detector at beam line elevation (left) and the schematic view of a typical drift-chamber sector (right) [35].

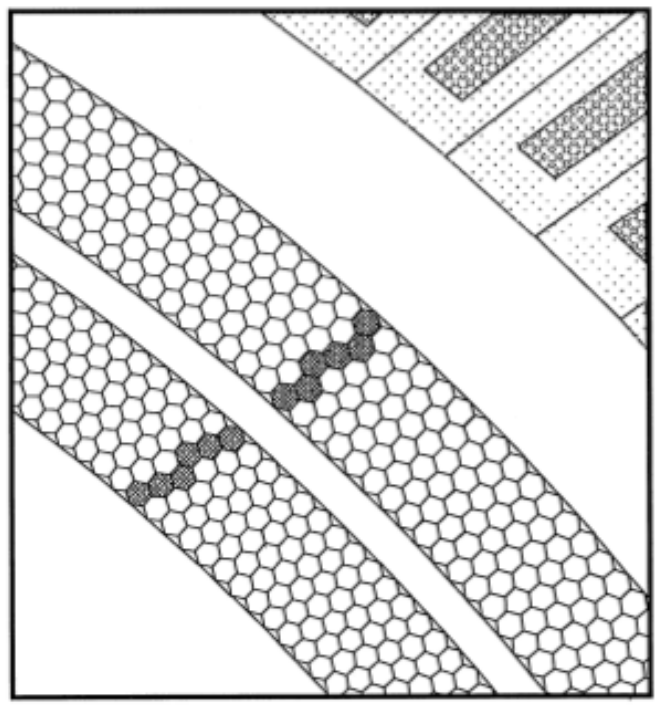

FIG. 37. A portion of the two super layers of R3 [35]. The sense wires are at the center of each hexagon and the field wires are at the vertices. 
range in azimuthal angle $\phi$. The TOF counters provide excellent timing resolution for particle identification. The TOF counters are also divided into six sectors. They are located after the DC, and Cherenkov counters and before the electromagnetic calorimeter. Each sector has four panels (see Fig. 38). Panel 1 (forward angle counters) includes counters $1-23$ and corresponds to scattering angles less than $45^{\circ}$. The forward angle counters are made of $15 \mathrm{~cm}$ wide scintillators and the large angle counters are made of $22 \mathrm{~cm}$ wide scintillators. The length of scintillators varies from $32-450 \mathrm{~cm}$. The $5.08 \mathrm{~cm}$ thickness of the scintillators gives a large signal for minimum ionizing particles compared to the background. All counters are fabricated from Bicron BC-408 scintillant and are instrumented with two PMTs, coupled to each end via light guides. In order to provide good timing resolution, two inch Thorn EMI 9954A PMTs were used for forward angle counters and three inch Philips XP432 B/D PMTs were used for large angle counters. The time resolution of the counters varies from about 80 ps for the shorter counters to up to 160 ps for the longer counters.

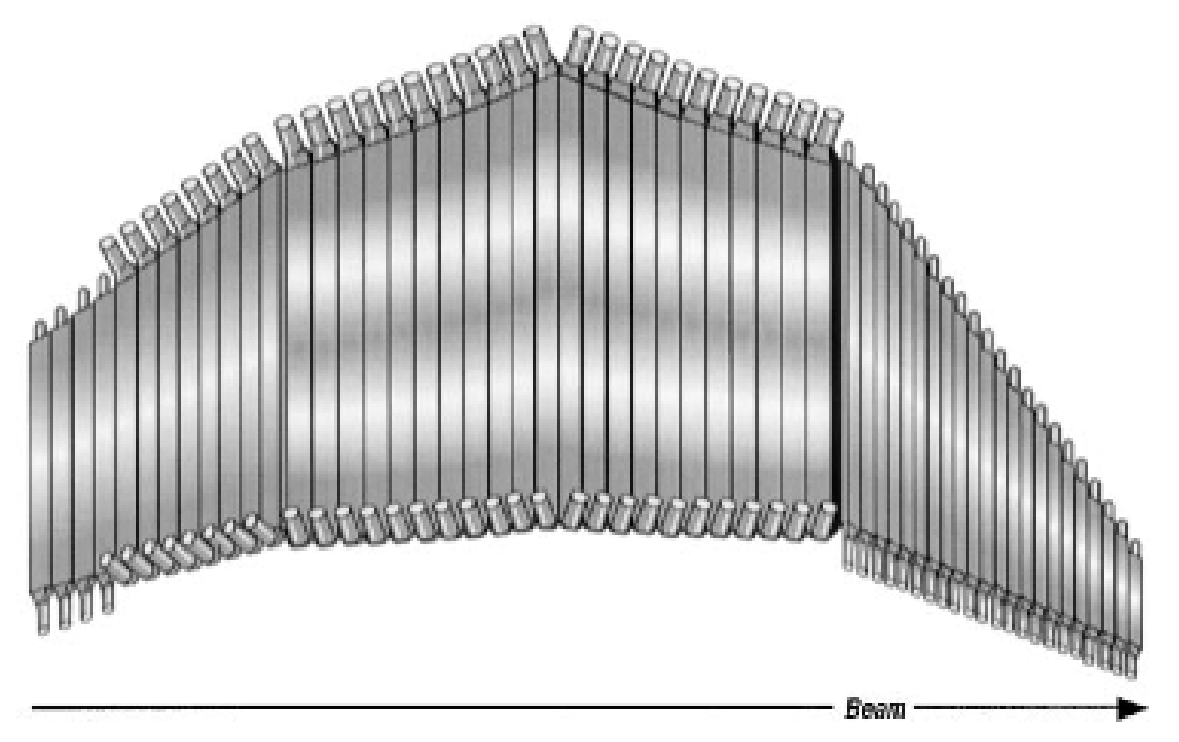

FIG. 38. The four panels of the TOF scintillator counters for one of the sectors. Scintillators have different light guides and PMTs for different scattering angle coverage [37].

As a charged particle passes through a scintillator, it excites a number of molecules 
which release a fraction of their energy as optical photons. This light is transmitted to the PMTs through the light guides. The optical signal is converted to an electric signal and amplified by PMTs and provides a prompt signal that can be used for the CLAS Level 1 trigger.

\subsubsection{ELECTROMAGNETIC CALORIMETER}

The main function of the CLAS electromagnetic calorimeter (EC) is to detect electrons, photons and neutrons. More specifically, the EC is capable of detecting and triggering electrons with energies above $0.5 \mathrm{GeV}$, detecting photons with energies above $0.2 \mathrm{GeV}$ and detecting neutrons, assuming their separation from photons based on timing information.

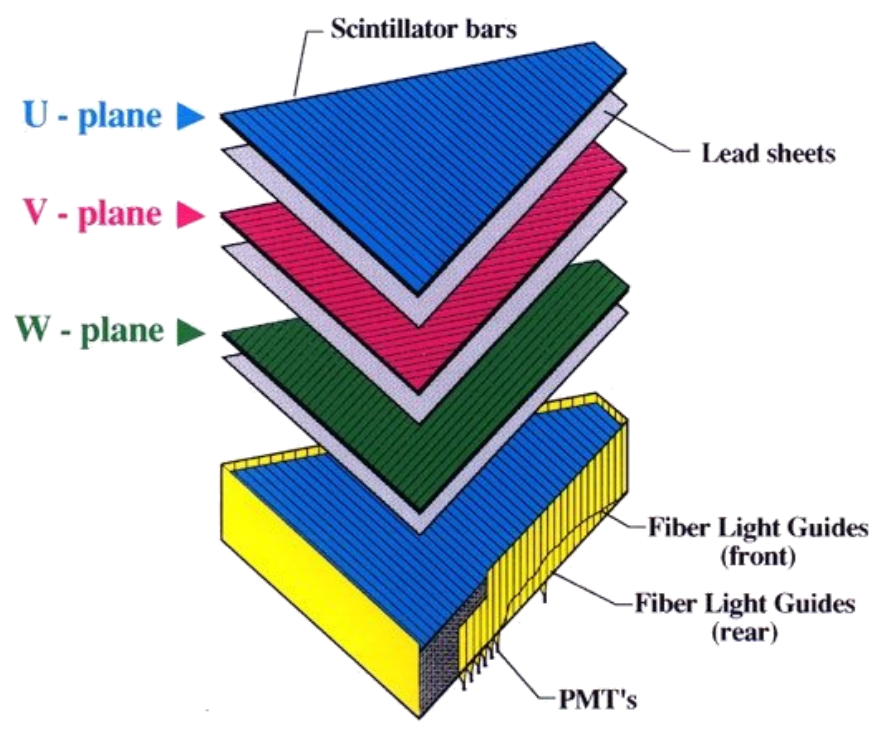

FIG. 39. One sector of the CLAS electromagnetic calorimeter [38]. The three layers of scintillator are placed in three different orientations, each rotated from the one above by $120^{\circ}$

The EC covers the forward region $\left(8^{\circ}<\theta<45^{\circ}\right)$ of each sector and is divided into six modules. Each module is shaped like an equilateral triangle, and consists of 39 layers of lead-scintillator sandwich with a total thickness of 16 radiation lengths. 
Each sandwich contains $10 \mathrm{~mm}$ thick BC412 scintillator and a $2.2 \mathrm{~mm}$ thick lead sheet. One scintillator layer is made of $3610 \mathrm{~cm}$ wide scintillators parallel to one side of the triangle. There are three layer orientations, one parallel to each side of the triangle, labeled as U, V and W, each containing 13 layers (see Fig. 39). These 13 layers of each orientation are grouped into an inner (5 layers) and outer (8 layers) stack to allow improved particle identification by longitudinally sampling the shower. Each module requires 36 (scintillators) $\times 3$ (orientations) $\times 2$ (stacks $)=216$ PMTs The scintillator light is transmitted to the PMTs by a fiber optic light read out system.

Because of its good energy and timing resolution, EC is used in the CLAS Level 1 trigger system. The average timing resolution of the entire detector is about 200 ps. More information on the design, construction, and performance of the EC can be found in Ref. [38].

\subsection{TRIGGER AND DATA ACQUISITION}

\subsubsection{TRIGGER}

To record the events of interest during the experiment, CLAS uses two-level trigger system. The Level 1 trigger processes any or all available prompt information from PMT channels such as locations of hits in the TOF, the signal in the CC and energy deposited in the EC to determine if a desired event has occurred. Signals from subsystems are compared with preloaded signals in memory tables for rapid response [32].

The signal from Level 1 and Level 2 triggers are then fed into a custom electronics board called "trigger supervisor (TS)". TS produces all common signals, gates and resets required by the detector electronics according to the trigger inputs. TS can be either operates as CLASS 1 which requires only Level 1 input or CLASS 2 which requires both Level 1 and Level 2 inputs. CLASS 1 trigger generates the gates upon receipt of any Level 1 input, waits for conversion of all crates to complete, and then places the event on a readout queue to initiate readout. In the CLASS2 trigger, again the TS generates the gates on Level 1 input, but then waits about $3.2 \mu$ s for a Level 2 confirmation. If Level 2 fails, TS sends a fast clear which causes all the electronics to reset and become active again. If Level 2 is satisfied, the front end modules will be allowed to convert, and the event will be placed on the readout queue for readout. 
Because the initial lepton energy is unknown in the TPE experiment, it is necessary to detect both the scattered lepton and the recoil proton to reconstruct the event. Thus the standard CLAS single-electron trigger was not appropriate for this measurement. The usual trigger which is based on CC and EC would cut out a lot of larger angle leptons and therefore limit the kinematic coverage. Another reason not to use the standard trigger is that CC has slightly different efficiencies for outbending and in-bending tracks. Instead, a new trigger was designed for the TPE experiment. The TPE trigger required a minimum ionizing hit in the EC and the forward TOF panel in one sector and a hit in any TOF detector in the opposite sector.

\subsubsection{DATA ACQUISITION}

The data of the various detector components corresponding to the events that pass through the experimental trigger are digitized and collected by 24 VME Readout Controllers. The digitized values are then translated into tables and each data value in these tables is associated with a unique component of the detector. In the next stage, the data arrays are sent to the CLAS online acquisition computer (CLON10) via fast ethernet lines. Three primary processes are performed on CLON10: Event Builder (EB), Event Transport (ET) and Event Recorder (ER). EB assembles incoming fragments into a complete event and labels them by run number, event number, and event type and arranges the data into "banks". These completed events are then passed to shared memory where the Event Transport (ET) allows them to be accessed by remote systems. Finally, ER collects those data and permanently stores them in magnetic media (RAID disks). The data files are transferred from RAID disks to the Jefferson Lab tape silo for offline analysis. 


\section{CHAPTER 4}

\section{DATA ANALYSIS}

This chapter will cover each step in the TPE data analysis process including detector calibration and data reduction, and other cuts and corrections.

\subsection{DATA COLLECTION}

The TPE experiment was carried out during November 2010 - February 2011. The electron beam of $5.5 \mathrm{GeV}$ from the CEBAF accelerator was used for all runs except the $2.2 \mathrm{GeV}$ straight-through runs. The mixed tertiary $e^{+} / e^{-}$beam was scattered from the $30 \mathrm{~cm}$ long liquid hydrogen target and scattered particles were detected using the CLAS. The primary trigger required a hit in EC in one sector and any TOF in the opposite sector. The nominal current for the torus magnet was $1500 \mathrm{~A}$ and the nominal current for the mini-torus magnet was $4000 \mathrm{~A}$. The pair spectrometer and Frascati dipoles were operated at $\sim 683 \mathrm{~A}$ and $\sim 327 \mathrm{~A}$, respectively. In order to reduce the systematic uncertainties, the torus magnet and chicane magnet currents were reversed several times during the run period. There were four possible configurations: positive torus-positive chicane, positive torus-negative chicane, negative tours-positive chicane and negative torus-negative chicane. The final data set was grouped into four magnet cycles and each magnet cycle contained data from all four configurations. A summary of the four magnet cycles is given in Table 2.

The data from the experiment were saved in separate files for each run, containing $\sim 20 \mathrm{M}$ events. This raw data was saved in BOS format. These BOS files were then processed by the CLAS reconstruction and analysis package (RECSIS) to identify the particles in the events. This process is called "cooking" and was performed by Robert Bennett for the TPE experiment.

\subsection{DETECTOR CALIBRATION AND DATA REDUCTION}

During the data collecting period, the experimental conditions were continuously monitored and all problems were recorded in a run sheet. Runs with potential problems were then carefully excluded from the data analysis. Problems included data 


\begin{tabular}{|l|c|c|c|c|}
\hline Cycle & Start Date & PS polarity & Torus polarity & \# of Events $(\mathrm{M})$ \\
\hline Cycle 1 & $12 / 8 / 2010$ & - & - & 519.4 \\
\hline & $12 / 13 / 2010$ & + & - & 507.7 \\
\hline & $12 / 16 / 2010$ & + & + & 949.4 \\
\hline \hline Cycle 2 & $1 / 20 / 2010$ & - & + & 265.1 \\
\hline & $1 / 11 / 2011$ & - & + & 824.5 \\
\hline & $1 / 14 / 2011$ & + & - & 708.3 \\
\hline & $1 / 19 / 2011$ & + & + & 775.4 \\
\hline \hline Cycle 3 & $1 / 23 / 2011$ & - & + & 723.4 \\
\hline & $1 / 29 / 2011$ & - & - & 770.3 \\
\hline & $2 / 2 / 2011$ & + & - & 739.3 \\
\hline & $2 / 5 / 2011$ & + & + & 768.1 \\
\hline \hline Cycle 4 & $2 / 8 / 2011$ & - & + & 748.5 \\
\hline & $2 / 11 / 2011$ & - & - & 797.2 \\
\hline & $2 / 15 / 2011$ & + & - & 932.7 \\
\hline & $2 / 19 / 2011$ & + & + & 391.2 \\
\hline & $2 / 15 / 2011$ & + & - & 821.0 \\
\hline & & & - & 197.6 \\
\hline
\end{tabular}

TABLE 2. Number of triggers collected in each magnet cycle for each configuration. 
acquisition problems, detector problems and beam quality problems. The information in the good runs were then reconstructed and converted into a "cooked" file that contains physical variables like time, position, energy, momentum, direction, etc. In addition to reconstruction of events, the cooking procedure also involved appropriate calibration of reconstructed data from different detector systems.

All detectors used in the experiment need to be properly calibrated to ensure that experimental quantities such as time or energy are correctly determined from the raw ADC and TDC information. The calibration might change from run to run. For example, the TOF calibration might change due to changes in the electronics or wiring; the DC electron drift velocity varies with changes in atmospheric pressure, temperature, humidity and gas mixture. In the TPE experiment, the data calibrations of the DC, TOF and EC were performed by Dipak Rimal, Puneet Khetarpal and Cole Smith respectively.

\subsubsection{TIME-OF-FLIGHT CALIBRATION}

Calibration of the TOF involves several steps. First, the raw TDC times $(T)$ are converted into hit times (in ns) by

$$
t=c_{0}+c_{1} T+c_{2} T^{2}+t_{\mathrm{walk}}
$$

where $c_{0}, c_{1}$ and $c_{2}$ are the constants to be determined and $t_{\text {walk }}$ is a correction factor for the instrumental shift in times created by pulses of varying heights. The remaining steps in the TOF calibration are as follows:

- Left-right delay constants: determine the correction needed to align the timing of left and right PMTs at each end of the same TOF paddle.

- Attenuation length: determine attenuation length of each counter.

- Effective velocity: for each paddle, determine the effective velocity of scintillator light, which can vary as the plastic ages and becomes less transparent.

- Paddle-to-paddle offsets: correct for shifts in the relative timing between paddles due to cable lengths or other factors.

- RF offset: delay time between the RF signal and the averaged event start time. 
Figure 40 shows the timing error (the measured ep time difference minus the expected $e p$ time difference) for elastic $e^{+} p$ and $e^{-} p$ events after the paddle-to-paddle offset corrections. The barely visible band on the negative side of the $\Delta t$ distribution seen in $e^{+} p$ events corresponds to events with incorrectly assigned (swapped) positron and proton. More information on the design and calibration of the TOF can be found in Ref. [37]. Paddle-to-paddle offset corrections were finalized by the author and more details will be discussed in Section 4.3.4.
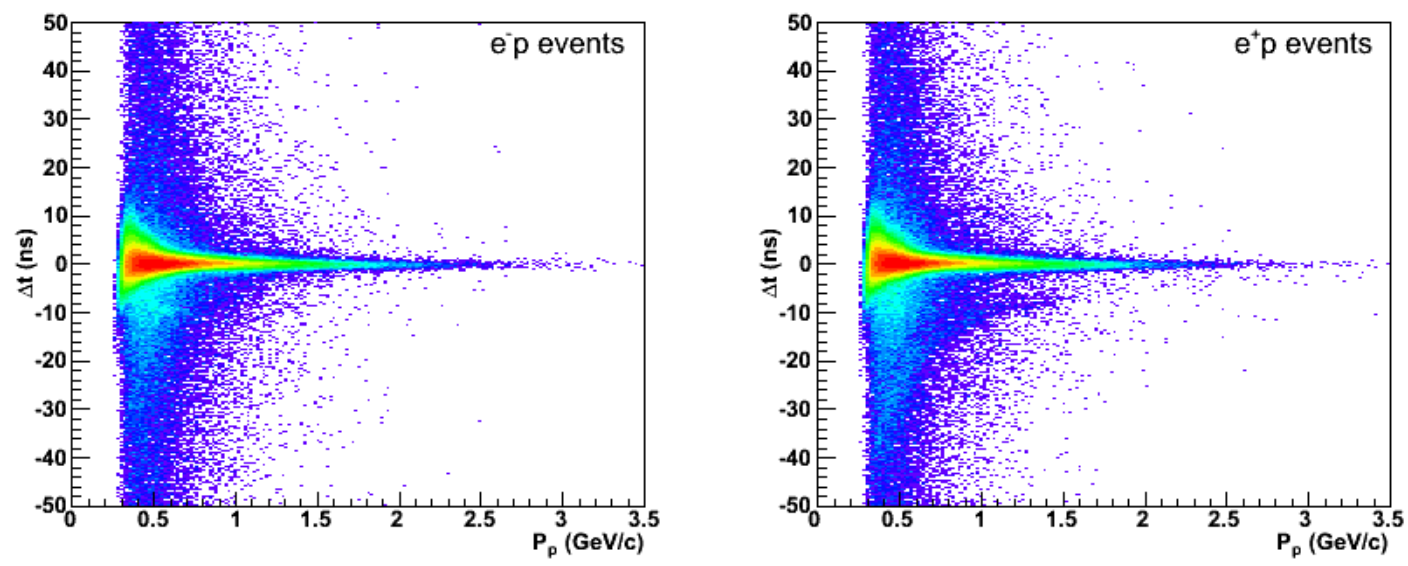

FIG. 40. The offset corrected $\Delta t$ as a function proton momentum, $p_{p}$ for $e^{-} p$ events (left) and $e^{+} p$ events (right).

\subsubsection{DRIFT CHAMBER CALIBRATION AND ALIGNMENT}

As described earlier, the drift chamber system consists of six identical sectors. Each sector is divided into three regions. Each region has two superlayers, axial and stereo. The schematic view of the Drift Chambers is shown in Fig. 16. Each superlayer contains six layers of sense wires except superlayer 1, which has only four layers. Each superlayer is separately calibrated, which yields 36 sets of calibration constants.

The main goal of the DC calibration is to optimize position measurements by refining the drift time to drift distance conversion process. The constants for the 
drift time to drift distance conversion are calibrated systematically and the stability over the run period is monitored. There are two stages of the reconstruction of the charged particles in the Drift Chambers. At the first stage, the individual tracks are fit to the hit-wire position and it is called hit based tracking (HBT). The resolution of the track momenta is comparatively poor $(3-5 \%)$ at this stage due to the size of the drift cells and the number of wire layers. The second stage is called time based tracking (TBT) where the timing information from the TOF and the measured drift times for each wire hit are used to calculate precise drift distances. The drift velocities, constants used to convert those corrected drift times to drift distances need to be calibrated.

The DC calibration is an iterative process and the initial values for the drift velocity parameters are taken from the previous experiment. A portion of the data was cooked with those parameters and the parameters are reoptimized. The process is reiterated until the drift velocity fit parameters converge. The parameters were adjusted to minimize the residual distributions for each super layer in each sector. The quality of calibration was monitored by plotting the mean and the width of the residual distributions as a function of run number. Figures 41 and 42 shows the means and widths of the residual distributions as a function of run number (time).

Alignment is also an important part of the DC calibration. To obtain a better resolution in momenta, we need to know the relative wire to wire positions in each drift chamber and the chamber to chamber positions relative to each other. Small chamber misalignments can have a large effect on the momentum resolution due to reconstructing an incorrect radius of curvature of a track. The TPE runs with torus magnetic field off (i.e. with straight particle tracks) were used for the alignment process.

More details about DC calibration and alignment can be found in Ref. [35].

\subsection{CUTS AND CORRECTIONS}

\subsubsection{ELASTIC EVENT SELECTION}

Because the TPE experiment did not have a monochromatic beam and because it could not use standard CLAS particle ID methods over the entire range of lepton angles, identifying elastic scattering events was challenging. The very first step in event selection was to select events with only two particles in the final state, either 


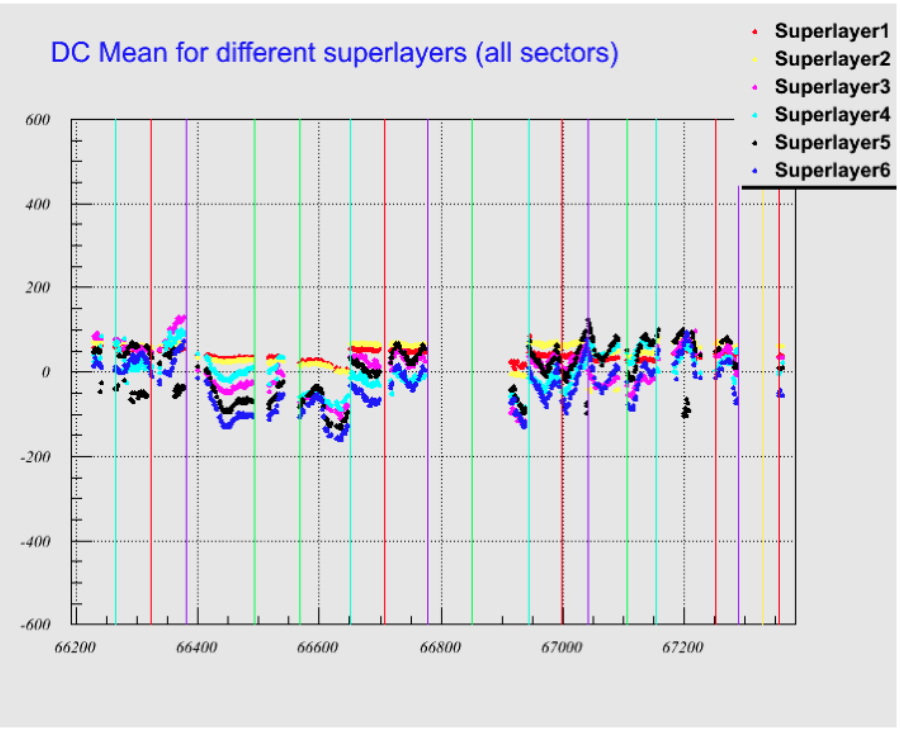

FIG. 41. Mean of the DC residual distribution vs. run number. The vertical lines on the plots show the magnet polarity reversals.

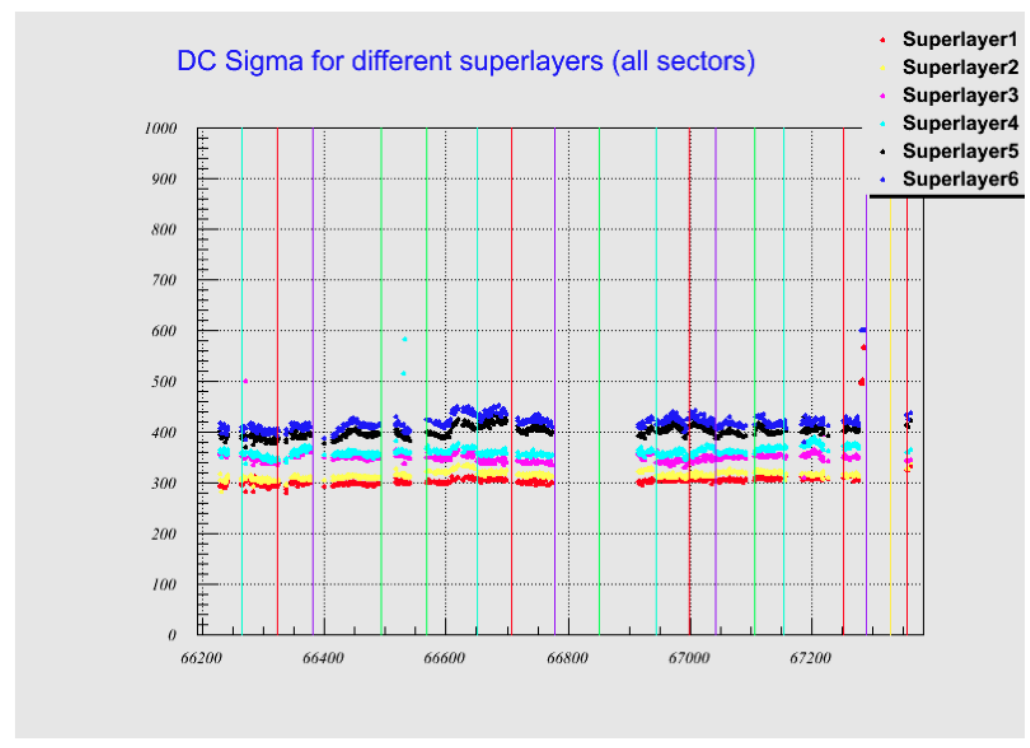

FIG. 42. Width (standard deviation) of a double gaussian fit to the DC residual distribution vs. run number. This is approximately equal to the single-layer DC resolution. 
positive-negative or positive-positive. In order to correctly identify the proton and the positron, we used the TOF timing information and the procedure is discussed in detail in timing corrections. The lepton's and proton's momenta $\left(p_{e}, p_{p}\right)$, scattering angles $\left(\theta_{e}, \theta_{p}\right)$ and azimuthal angles $\left(\phi_{e}, \phi_{p}\right)$, were used as described in the following sections to eliminate the non-elastic events and background.

\section{Elastic Kinematic Cuts}

Four elastic kinematic cuts were applied to the TPE data.

1. Azimuthal angle difference $(\Delta \phi)$

The difference between the lepton and proton azimuthal angles, $\Delta \phi=\phi_{e}-\phi_{l}$.

2. Incident lepton energy difference $\left(\Delta E_{\text {Incident }}\right)$

Even though the incident lepton energy is not measured in the experiment, it could be reconstructed using the measured scattered lepton and proton momenta in two different way as shown in Eqs. (52) and (53).

$$
\begin{aligned}
E_{\text {Incident }}^{1} & =M_{p}\left(\cot \frac{\theta_{e}}{2} \cot \theta_{p}-1\right) \\
E_{\text {Incident }}^{2} & =p_{e} \cos \theta_{e}+p_{p} \cos \theta_{p} \\
\Delta E_{\text {Incident }} & =E_{\text {Incident }}^{1}-E_{\text {Incident }}^{2}
\end{aligned}
$$

3. Scattered lepton energy difference $\left(\Delta E_{e}\right)$

The energy of the scattered lepton can be directly measured, $E_{e}$, or it can be calculated from the electron scattering angle and incident energy as Eq. (54). The incident energy was calculated from the scattering angles, $E_{\text {Incident }}^{1}$.

$$
\begin{aligned}
E_{e} \text { calculated } & =\frac{M_{p} E_{\text {Incident }}^{1}}{E_{\text {Incident }}^{1}\left(1-\cos \theta_{e}\right)+M_{p}} \\
\Delta E_{e} & =E_{e}-E_{e}^{\text {calculated }}
\end{aligned}
$$

4. Recoiling proton momentum difference $\left(\Delta p_{p}\right)$

The momentum of the recoiling proton can be directly measured or it can be calculated using other measured quantities as shown in Eq. (55).

$$
\begin{aligned}
p_{p}{ }^{\text {calculated }} & =\frac{E_{e} \sin \theta_{e}}{\sin \theta_{p}} \\
\Delta p_{p} & =p_{p}-p_{p}{ }^{\text {calculated }}
\end{aligned}
$$



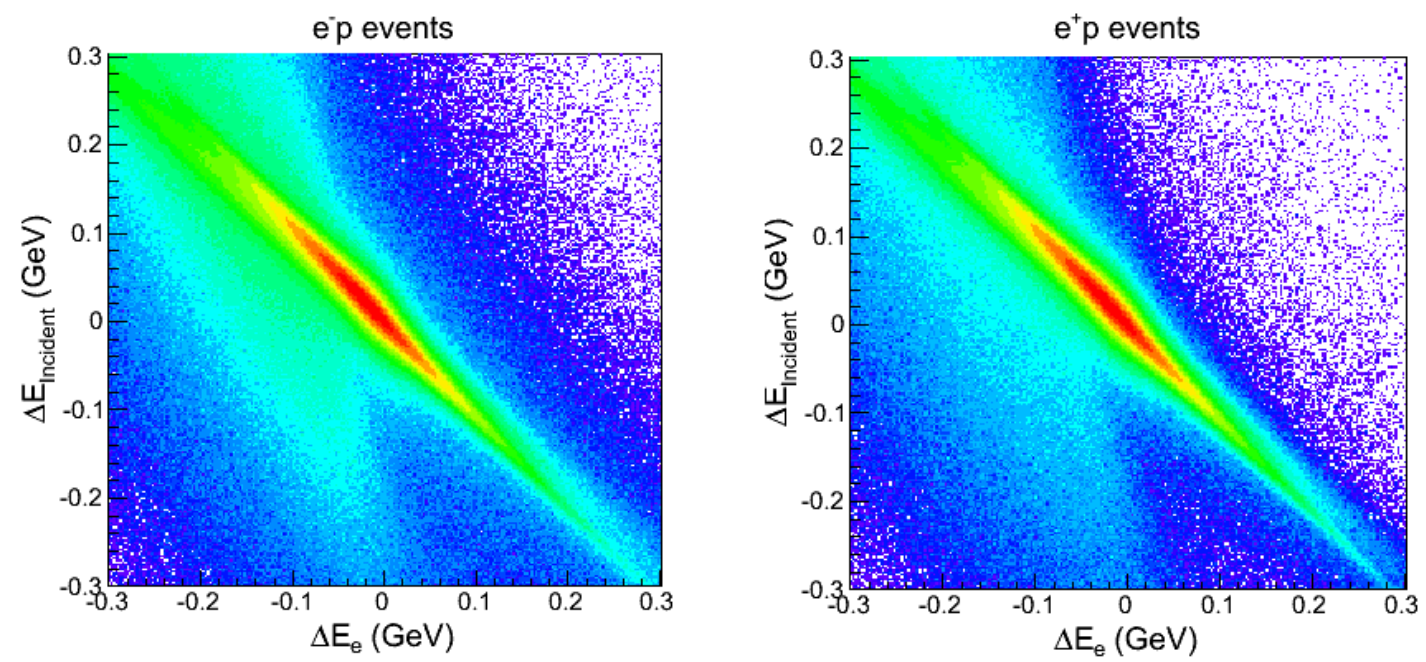

FIG. 43. $\Delta E_{\text {Incident }}$ vs. $\Delta E_{e}$ distributions for $e^{-} p$ events and $e^{+} p$ events.

Figure 43 shows that there is a strong correlation between $\Delta E_{e}$ and $\Delta E_{\text {Incident }}$. Based on this correlation, a new set of cut variables were introduced as below.

$$
\begin{aligned}
& \Delta E^{+}=\Delta E_{\text {Incident }}+\Delta E_{e} \\
& \Delta E^{-}=\Delta E_{\text {Incident }}-\Delta E_{e}
\end{aligned}
$$

Figure $44,45,46$ and 47 show the distributions of $\Delta \phi, \Delta p_{p}$ and $\Delta E^{ \pm}$for different bins in $Q^{2}$ and $\varepsilon$. Each distribution is cut on the peaks of the other three to select elastic scattering events. The distributions are very clean except for low $\varepsilon$ and high $Q^{2}$. Background subtraction is discussed in Section 4.3.9. The widths of the distributions of $\Delta \phi, \Delta p_{p}$ and $\Delta E^{ \pm}$vary noticeably with $Q^{2}$ and $\varepsilon$. Figures 48 and 49 show that the widths of the Gaussian distributions fitted to peaks of the $\Delta E^{+}$ distribution increase with $Q^{2}$, but that there is no significant difference between the distributions for $e^{-} p$ and $e^{+} p$ events or for the different torus magnet settings. We therefore summed the $e^{-} p$ and $e^{+} p$ events for both torus polarities to determine the elastic scattering cut parameters.

Based on the means and widths of the fitted gaussian distributions to the peaks, $Q^{2}$ and $\varepsilon$ dependent, parameterized cuts covering the full $Q^{2}-\varepsilon$ range were determined for all four kinematic variables. The $Q^{2}$ dependence of the means and the widths of Gaussian functions fitted to the four variables are shown in Figs. 50, 51, 52 and 53. 

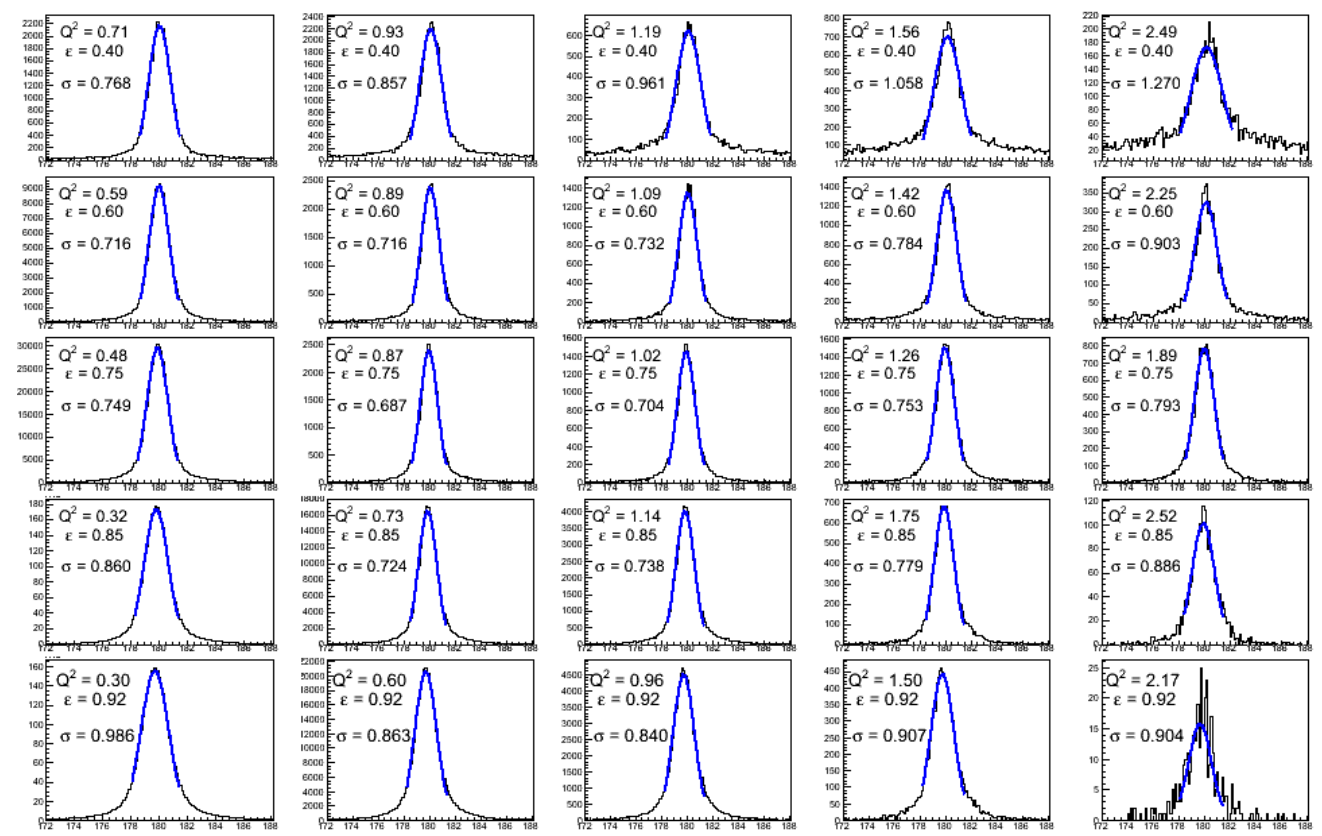

FIG. 44. $\Delta \phi\left(^{\circ}\right)$ distributions for different $Q^{2}$ and $\varepsilon$ bins. The horizontal axis ranges from $172^{\circ}$ to $188^{\circ}$.
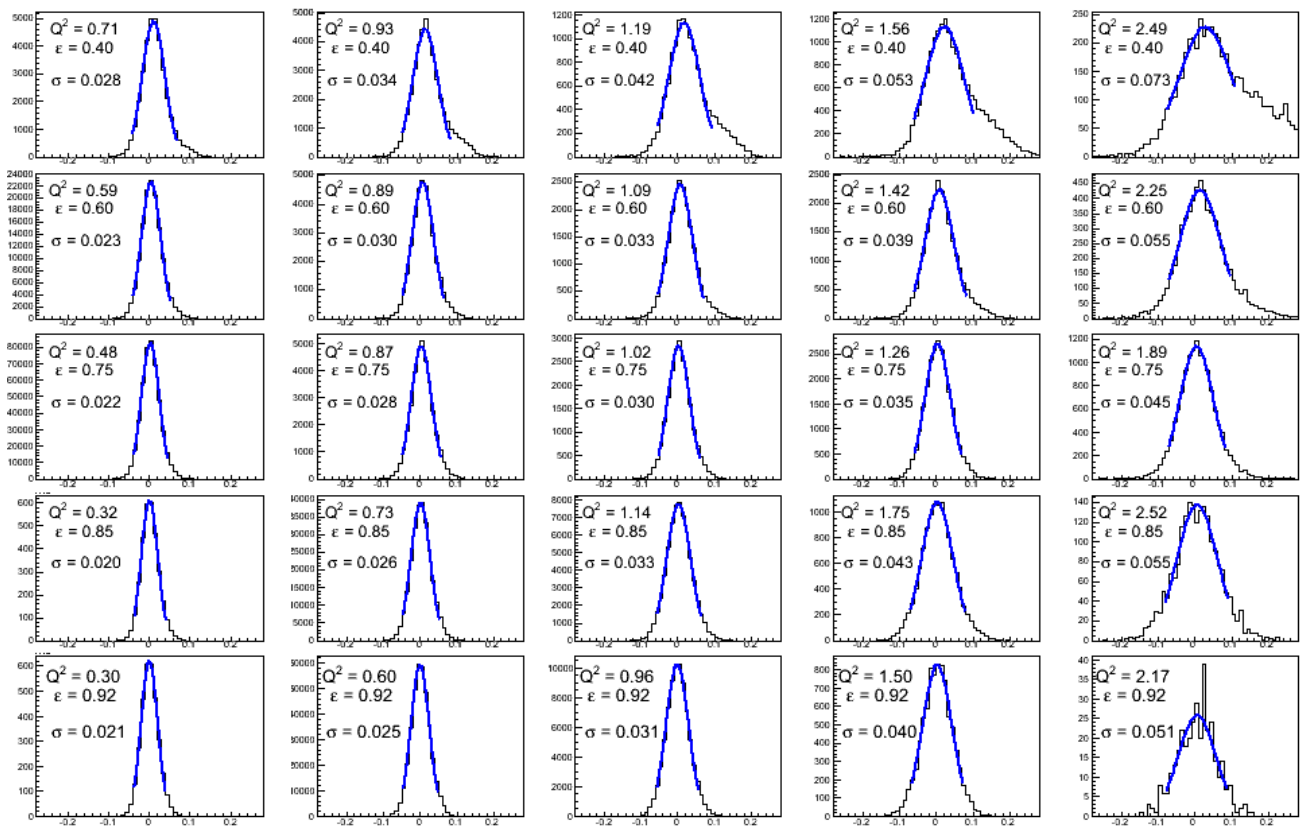

FIG. 45. $\Delta p_{p}(\mathrm{GeV})$ distributions for different $Q^{2}$ and $\varepsilon$ bins. The horizontal axis ranges from $-0.3 \mathrm{GeV} / \mathrm{c}$ to $0.3 \mathrm{GeV} / \mathrm{c}$. 

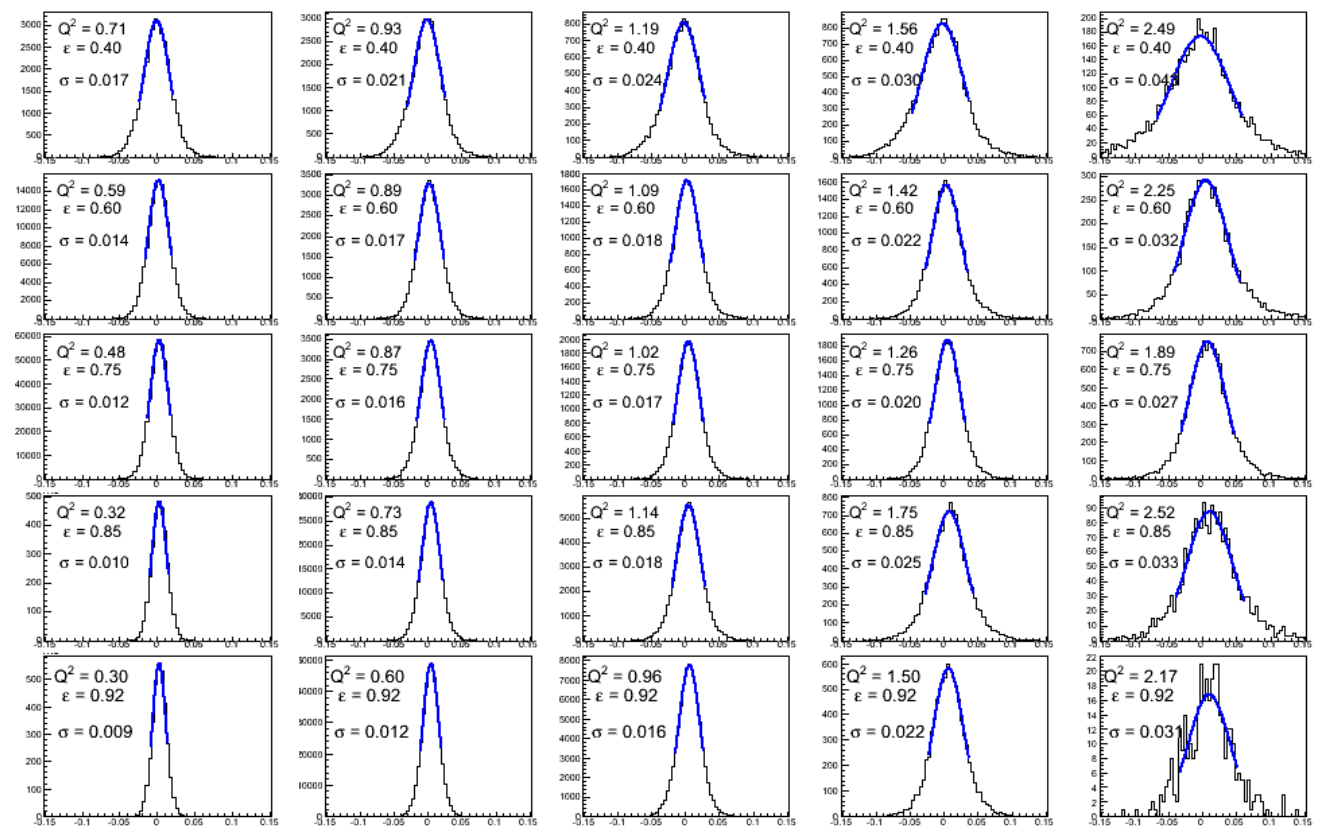

FIG. 46. $\Delta E^{+}(\mathrm{GeV})$ distributions for different $Q^{2}$ and $\varepsilon$ bins. The horizontal axis ranges from $-0.15 \mathrm{GeV}$ to $0.15 \mathrm{GeV}$.
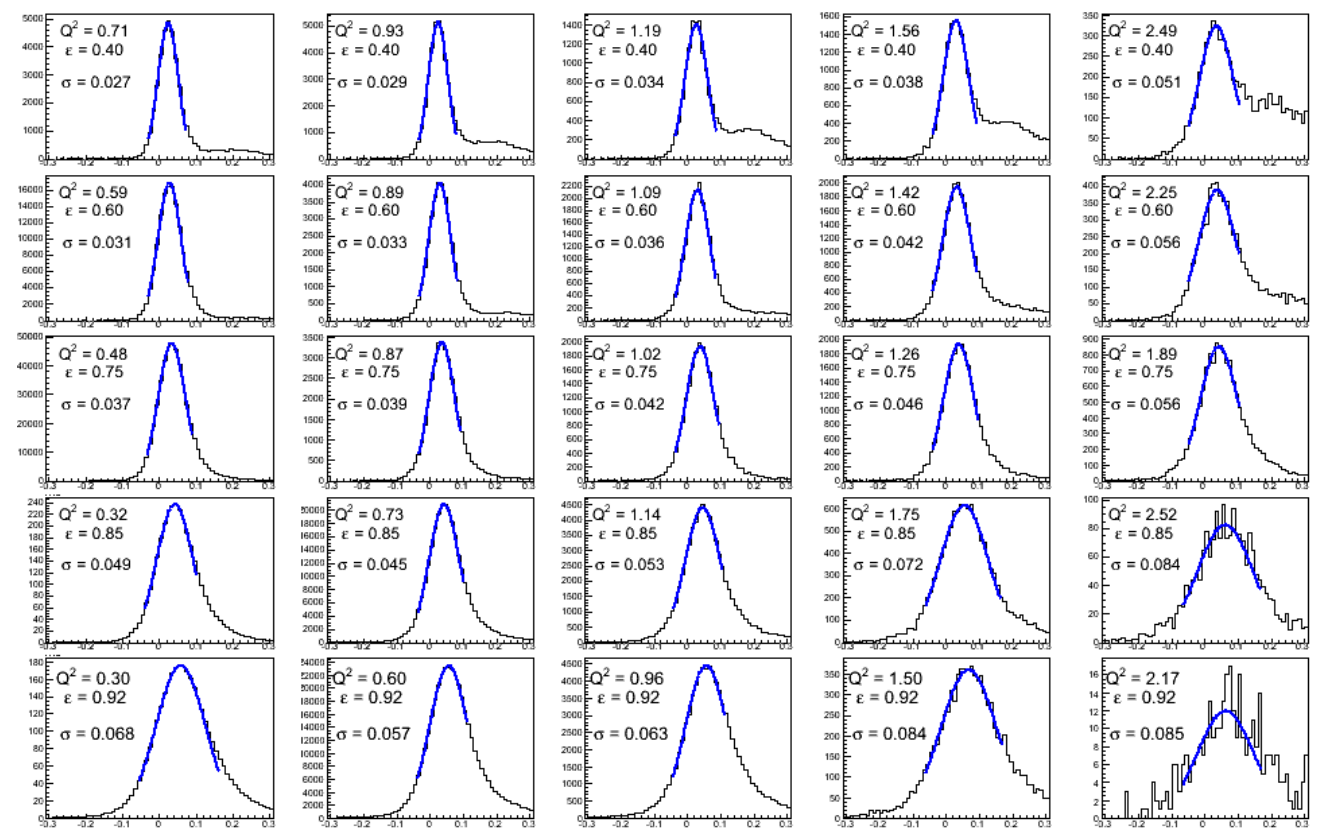

FIG. 47. $\Delta E^{-}(\mathrm{GeV})$ distributions for different $Q^{2}$ and $\varepsilon$ bins. The horizontal axis ranges from $-0.3 \mathrm{GeV}$ to $0.3 \mathrm{GeV}$. 
Figure 54 shows the distributions of $\Delta \phi, \Delta p_{p}$ and $\Delta E^{ \pm}$before and after applying the other three cuts on each. The differences of these parameterized cuts for the four different magnet configurations were studied but those differences were negligible. The four kinematic cuts were applied to each of the four distributions to select elastic events. The cuts were centered at the mean of the distribution with widths ranging from three to four standard deviations to each side. These cuts varied event-by-event, rather than bin-by-bin, because some of the $Q^{2}$ and $\varepsilon$ bins were very large. The effects of varying the widths of these cuts were carefully studied and are described under systematic uncertainties.

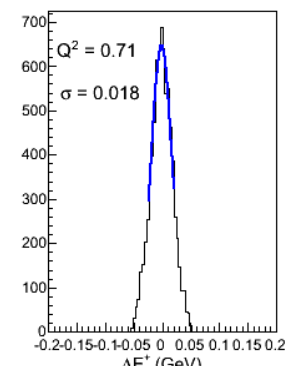

$\Delta \mathrm{E}^{+}(\mathrm{GeV})$

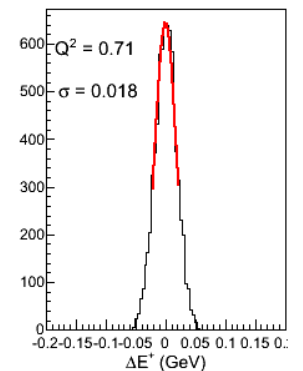

$\Delta \mathrm{E}^{+}(\mathrm{GeV})$

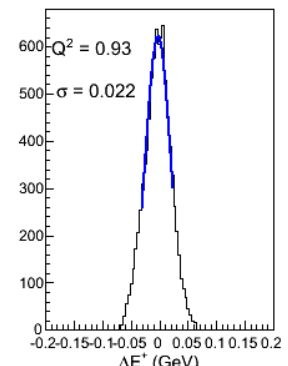

$\Delta \mathrm{E}^{+}(\mathrm{GeV})$

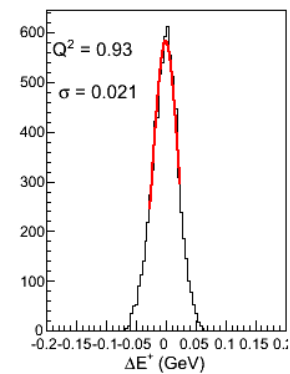

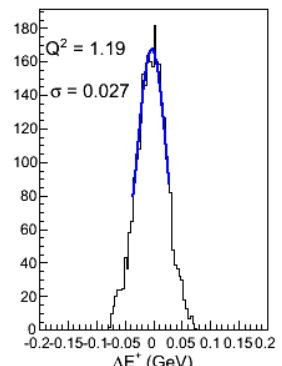

$\Delta \mathrm{E}^{+}(\mathrm{GeV})$

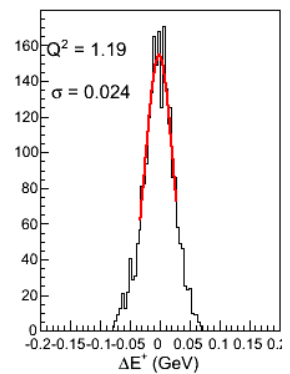

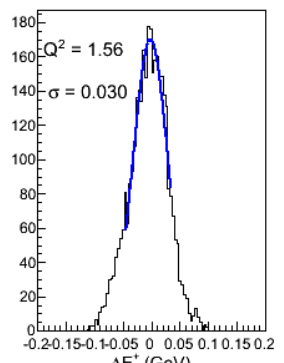

$\Delta \mathrm{E}^{+}(\mathrm{GeV})$

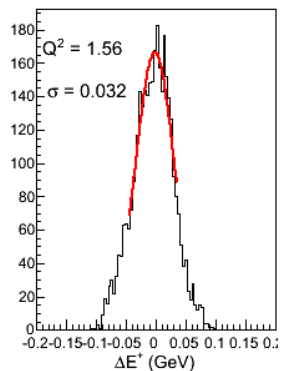

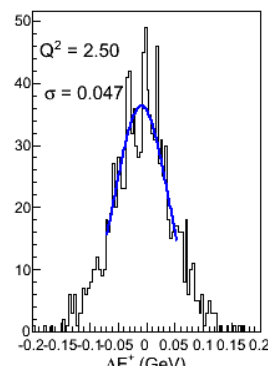

$\Delta \mathrm{E}^{+}(\mathrm{GeV})$

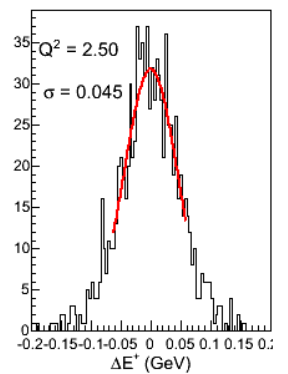

FIG. 48. $\Delta E^{+}$distributions for different $Q^{2}$ bins for $0.1<\varepsilon<0.5$ with positive torus magnet setting, top row for $e^{-} p$ events and bottom row for $e^{+} p$ events.

\subsubsection{MOMENTUM CORRECTIONS}

Drift chamber misalignment and inaccurate magnetic field maps lead to systematic deviations in reconstructed momenta in CLAS. This is reflected in shifted and broadened missing and invariant mass distributions. Figure 55 shows that the centroid of the invariant mass distribution of the $\mathrm{H}\left(e, e^{\prime}\right)$ elastic peak is shifted from the 

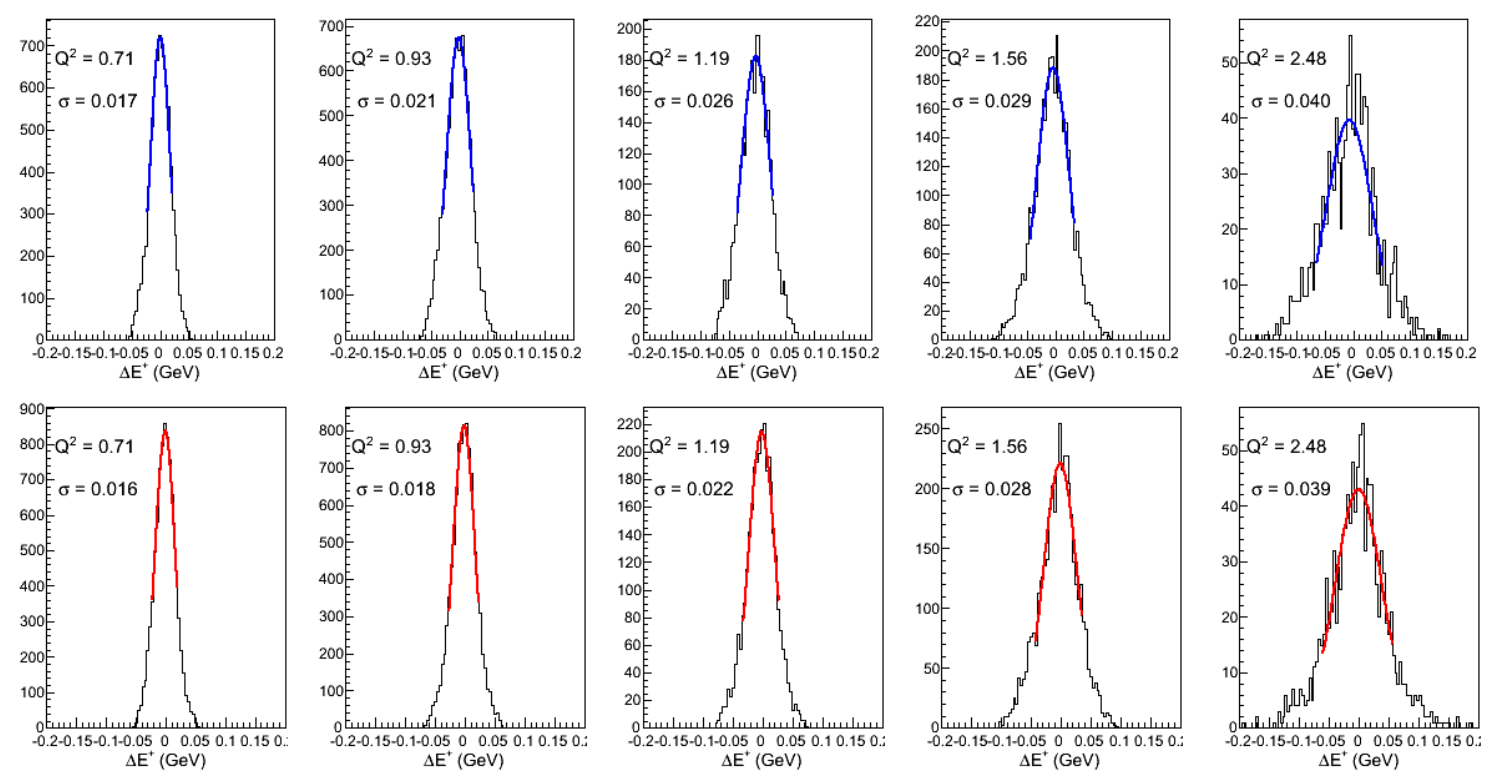

FIG. 49. $\Delta E^{+}$distributions for different $Q^{2}$ bins for $0.1<\varepsilon<0.5$ with negative torus magnet setting, top row for $e^{-} p$ events and bottom row for $e^{+} p$ events.
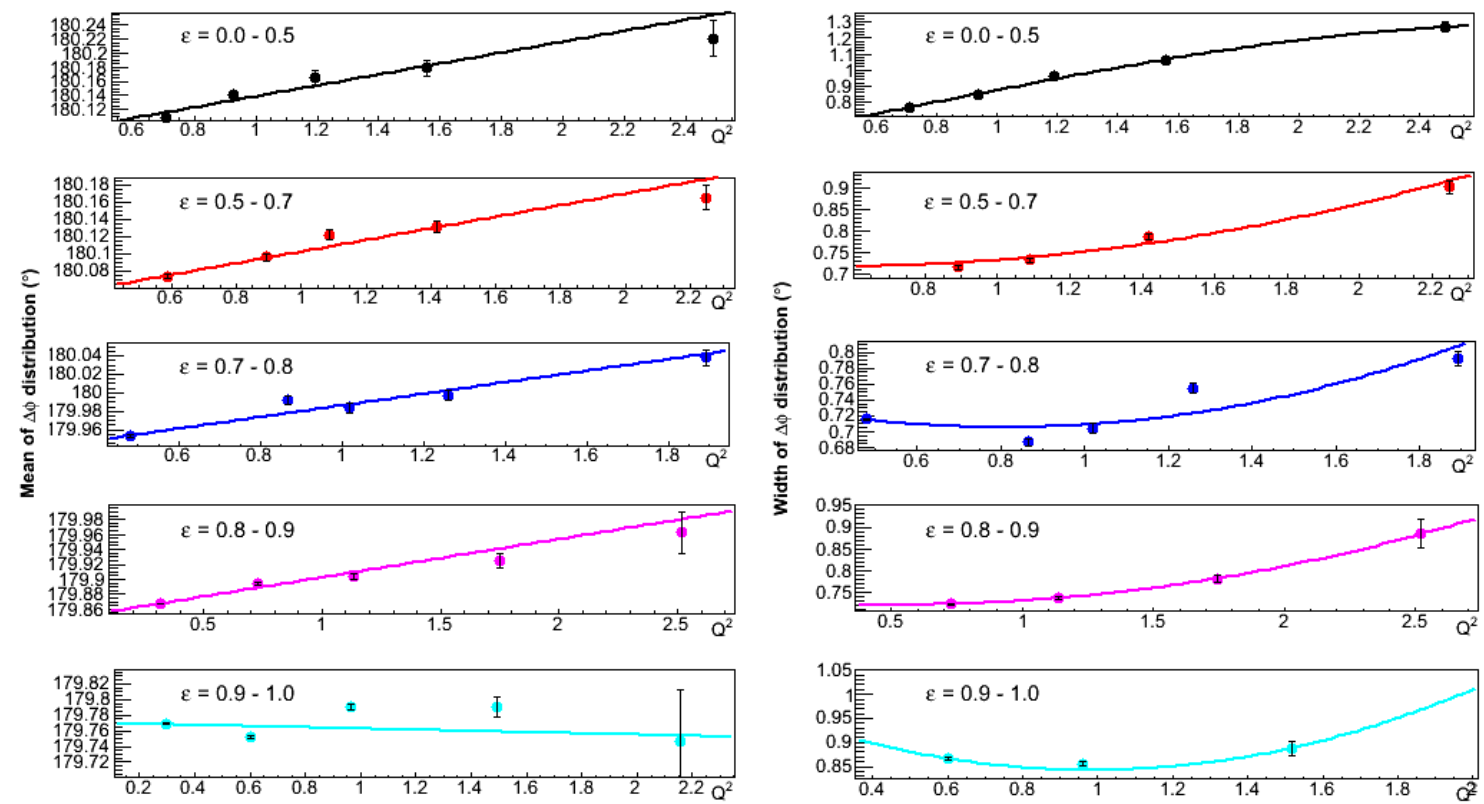

FIG. 50. $Q^{2}$ dependence of the means (left) and the widths (right) of the Gaussian functions fit to $\Delta \phi$ distributions for different $\varepsilon$ ranges. The horizontal scales differ. 

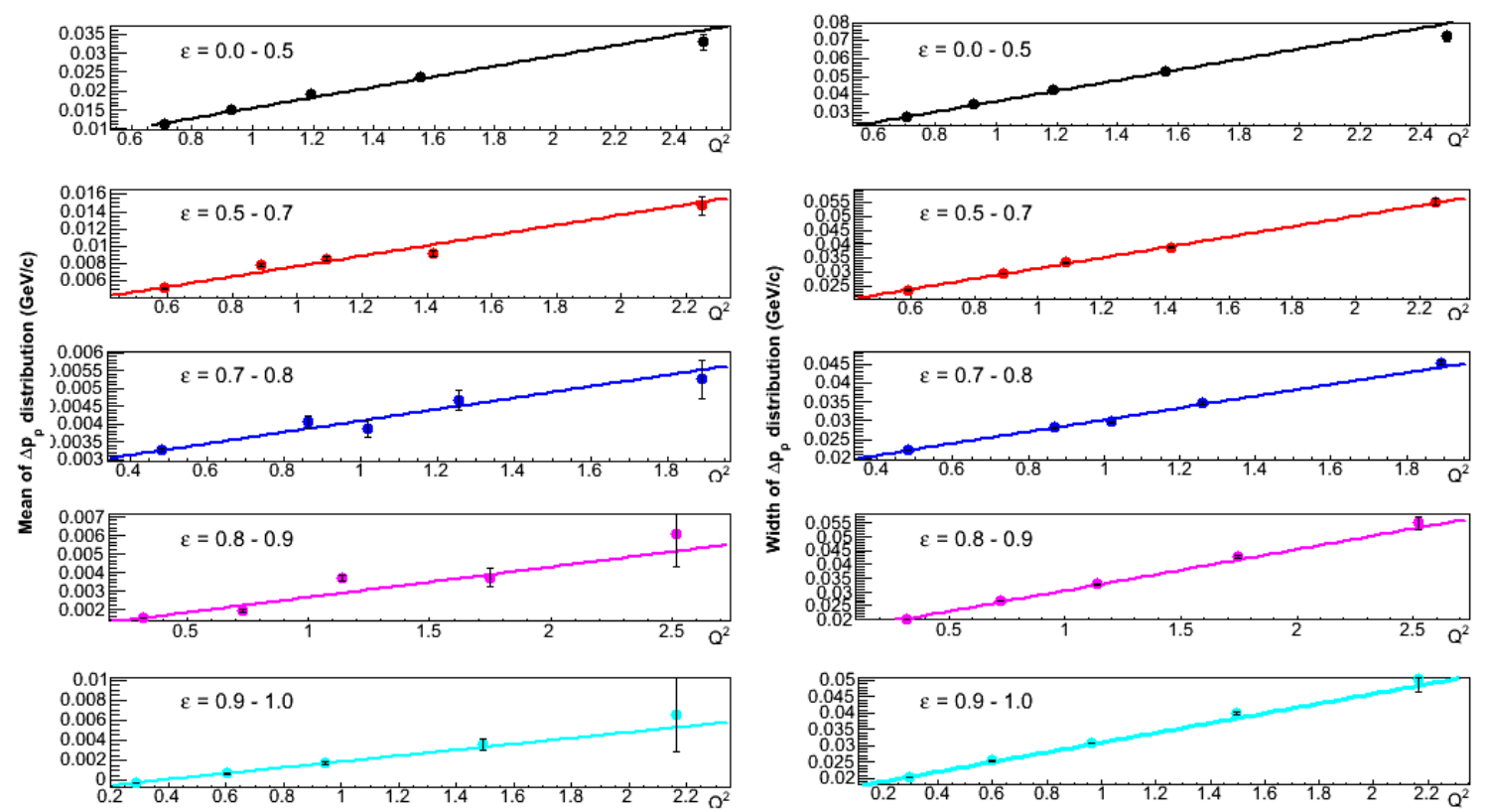

FIG. 51. $Q^{2}$ dependence of the means (left) and the widths (right) of the Gaussian functions fit to $\Delta p_{p}$ distributions for different $\varepsilon$ ranges. The horizontal scales differ.
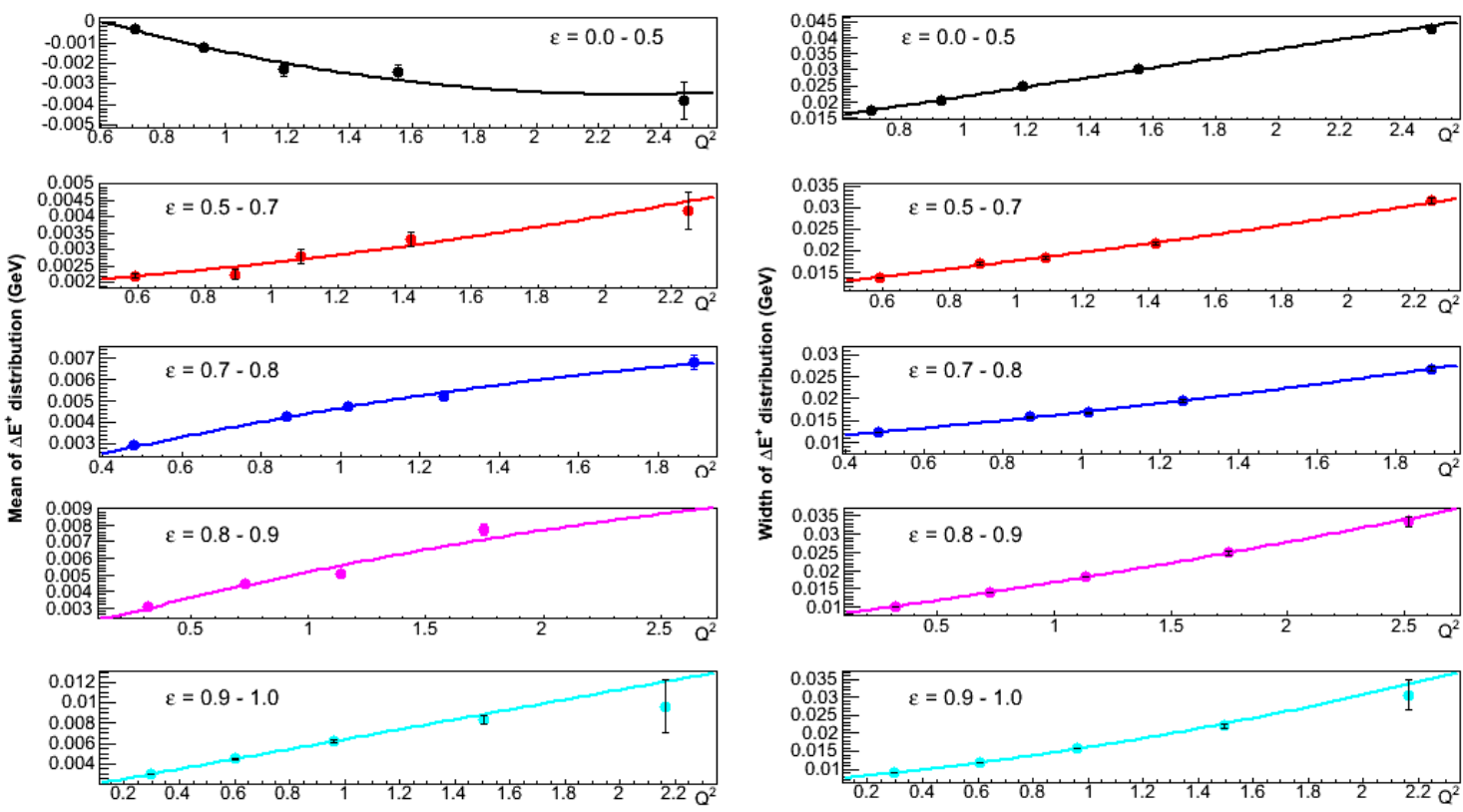

FIG. 52. $Q^{2}$ dependence of the means (left) and the widths (right) of the Gaussian functions fit to $\Delta E^{+}$distributions for different $\varepsilon$ ranges. The horizontal scales differ. 

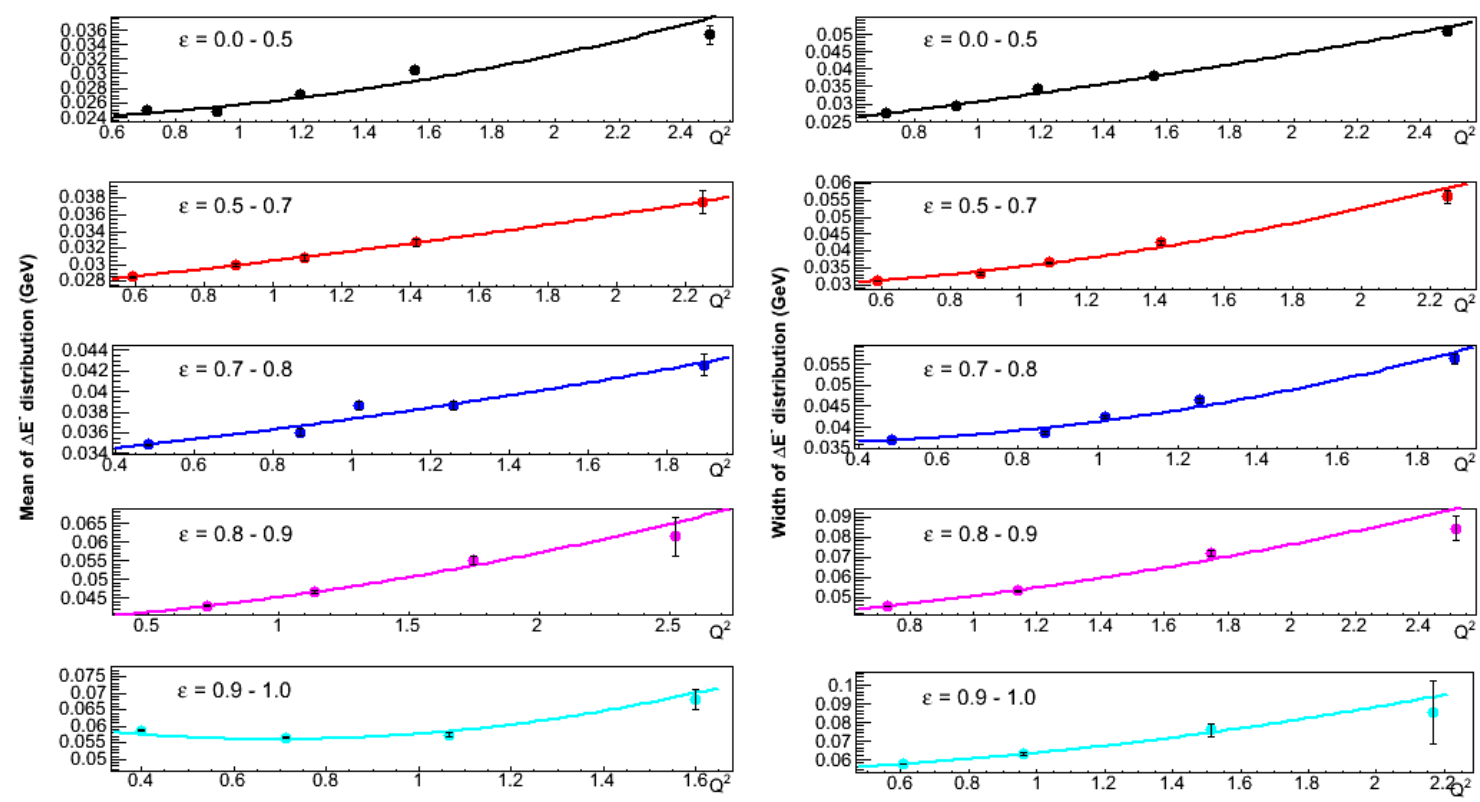

FIG. 53. $Q^{2}$ dependence of the means (left) and the widths (right) of the Gaussian functions fit to $\Delta E^{-}$distributions for different $\varepsilon$ ranges. The horizontal scales differ.
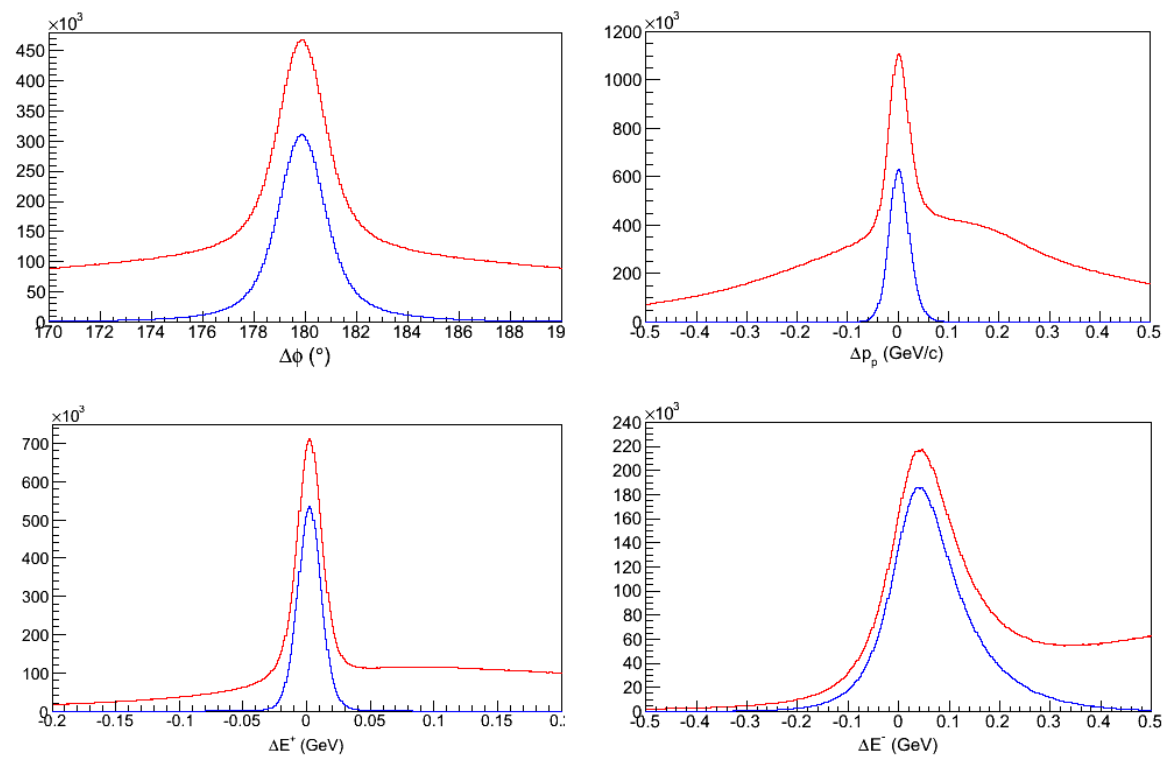

FIG. 54. The four variables, $\Delta \phi, \Delta p_{p}$ and $\Delta E^{ \pm}$, before (red) and after (blue) applying the other three elastic cuts on each. The width of the elastic cuts used are $3 \sigma$. 
proton mass, $M_{p}=0.938 \mathrm{GeV}$. The width of the centroid is also significantly broader than expected from the intrinsic momentum resolution of CLAS.

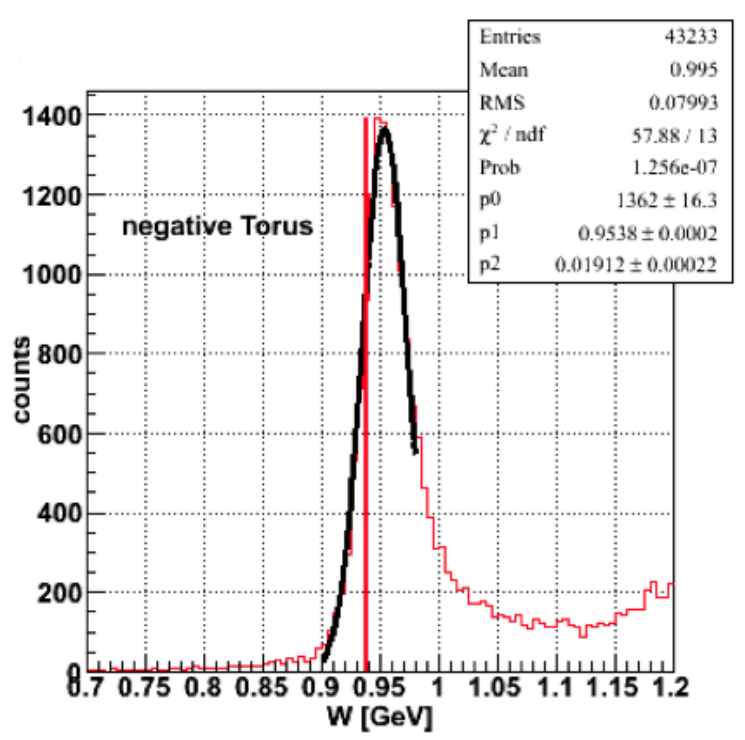

FIG. 55. Invariant mass distribution of the $\mathrm{H}\left(e, e^{\prime}\right)$ elastic peak for $2.2 \mathrm{GeV}$ incident electrons before momentum correction, data (red) and the gaussian fit (black). The vertical line is the proton mass $M_{p}=0.938 \mathrm{GeV}^{2}$.

In order to correct the particle momenta and angles, we require precise knowledge of the incident lepton energy. In addition to the TPE production runs which have a very broad range of tertiary lepton energies, there were a few special runs with 2258 $\mathrm{MeV}$ primary electrons incident on the target. Those special runs were analyzed and used to calculate the required momentum corrections. The momentum and angle corrections for TPE data analysis were done by Dipak Rimal [39] following the method used by the CLAS e6 run group [40].

We compared the measured electron momentum and that calculated from the incident energy and scattering angle. Figure 56 shows that the difference between the measured and calculated electron momentum depends on $\phi$. The correction procedure assumes that both momenta and polar angles are distorted by systematic displacements of the drift chambers and by magnetic field deviations from the field 

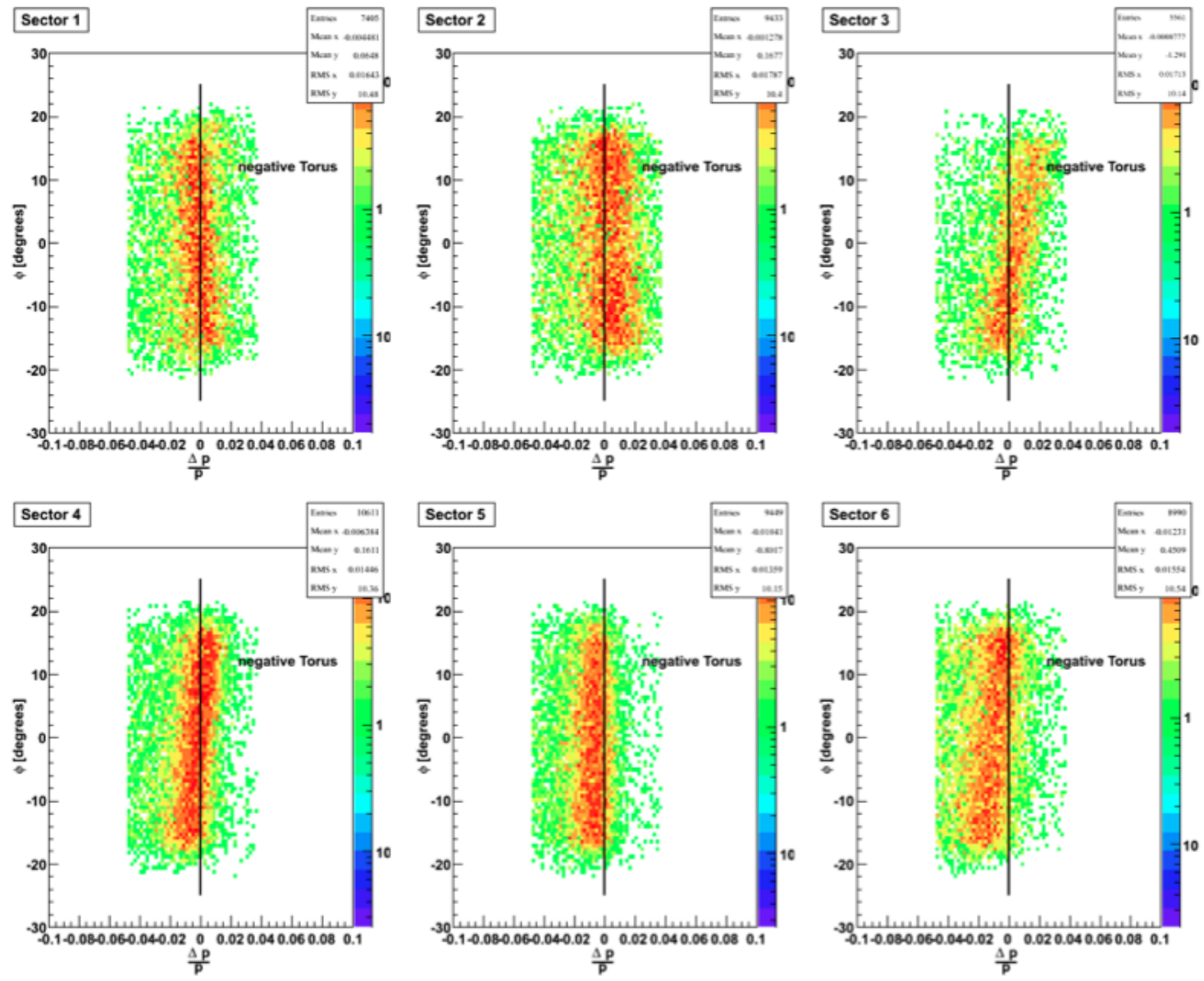

FIG. 56. Before momentum corrections: relative difference between the measured and calculated electron momentum $(\Delta p / p)$ vs. azimuthal angle $\phi$ for elastic scattering for all six sectors of CLAS. 
map used in the reconstruction code. The deviations of measured values from expected, $\Delta \theta$ in the polar scattering angle, and $\Delta p / p$ in momentum, are minimized by adjusting eight free parameters for each sector which represent the displacements and rotations of the $R 2$ and $R 3$ drift chambers and six more parameters correcting the effects of inaccurate magnetic field maps. A detailed description of the procedure can be found in Ref. [40]. Figure 57 shows the $\Delta p / p$ vs. $\phi$ distributions after momentum and angular corrections. The $\Delta p / p$ distributions no longer vary with $\phi$.
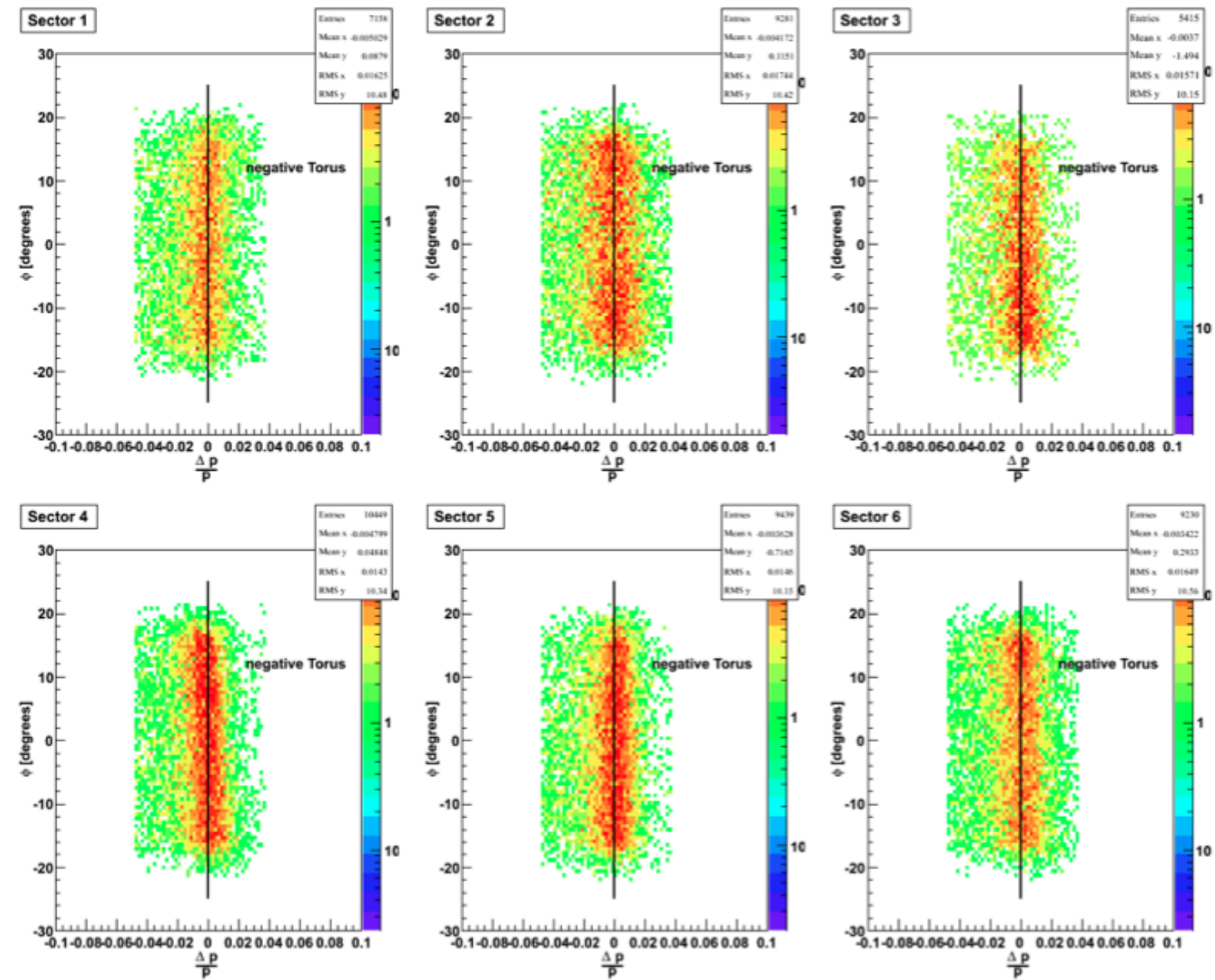

FIG. 57. After momentum corrections: relative difference between measured and calculated electron momentum $(\Delta p / p)$ vs. azimuthal angle $\phi$ for $2.2 \mathrm{GeV}$ elastic scattering events. 


\subsubsection{ENERGY LOSS CORRECTIONS}

Charged particles lose energy while propagating through target and detector material, mainly by ionizing atoms they pass near. The energy loss is more significant for lower momentum and heavier particles. This process is described by the wellknown Bethe-Bloch formula. Due to this process, the reconstructed momentum seen in the Drift chambers is actually less than the momentum of the proton at the interaction point. In order to correct the energy loss of each detected charged particle in TPE data, the CLAS Energy Loss correction software package (ELoss) was used during the data analysis. The geometry of the TPE target and target materials were inserted into the ELoss package correctly so that it considered all the material which the charged particles passed through. In general, these energy loss corrections are on the order of several $\mathrm{MeV}$, but for low momentum protons these energy loss corrections are quite substantial.

\subsubsection{TIMING OFFSET CORRECTIONS}

We need to correctly identify the positron and the proton for the two positive track events. For the one positive-one negative track events, the lepton (electron) can be easily identified from the charge. This section describes how the timing information of the two particles was used to differentiate the positron and the proton. The difference between the measured time and the calculated time, $\Delta t$, for each particle and for an event can be calculated as follows:

$$
\begin{aligned}
\Delta t_{\text {Lepton }} & =t_{\text {TOF }}-\frac{l_{\text {Lepton }}}{c} \\
\Delta t_{\text {Proton }} & =t_{\text {TOF }} \\
\Delta \text { Proton } & -\frac{l_{\text {Proton }}}{\beta c} \\
\Delta t & =\Delta t_{\text {Proton }}-\Delta t_{\text {Lepton }}
\end{aligned}
$$

where $t_{T O F}$ is the track time measured at the TOF scintillators, $l$ is the distance travelled by the particle from the event vertex to the paddle hit position, $\beta=\frac{p c}{E}$, and $E$ and $p$ are the energy and momentum of the particle. $t_{T O F}$ depends on which particle was selected by the reconstruction code as the trigger particle. $\Delta t$ does not. Figure 58 shows the $\Delta t$ distribution of the elastic electron-proton events as a function of proton momentum for the four magnet cycles. The offset horizontal bands indicate miscalibrated TOF scintillators. Magnet cycle 1 had a different set of miscalibrated scintillators than the other cycles. 

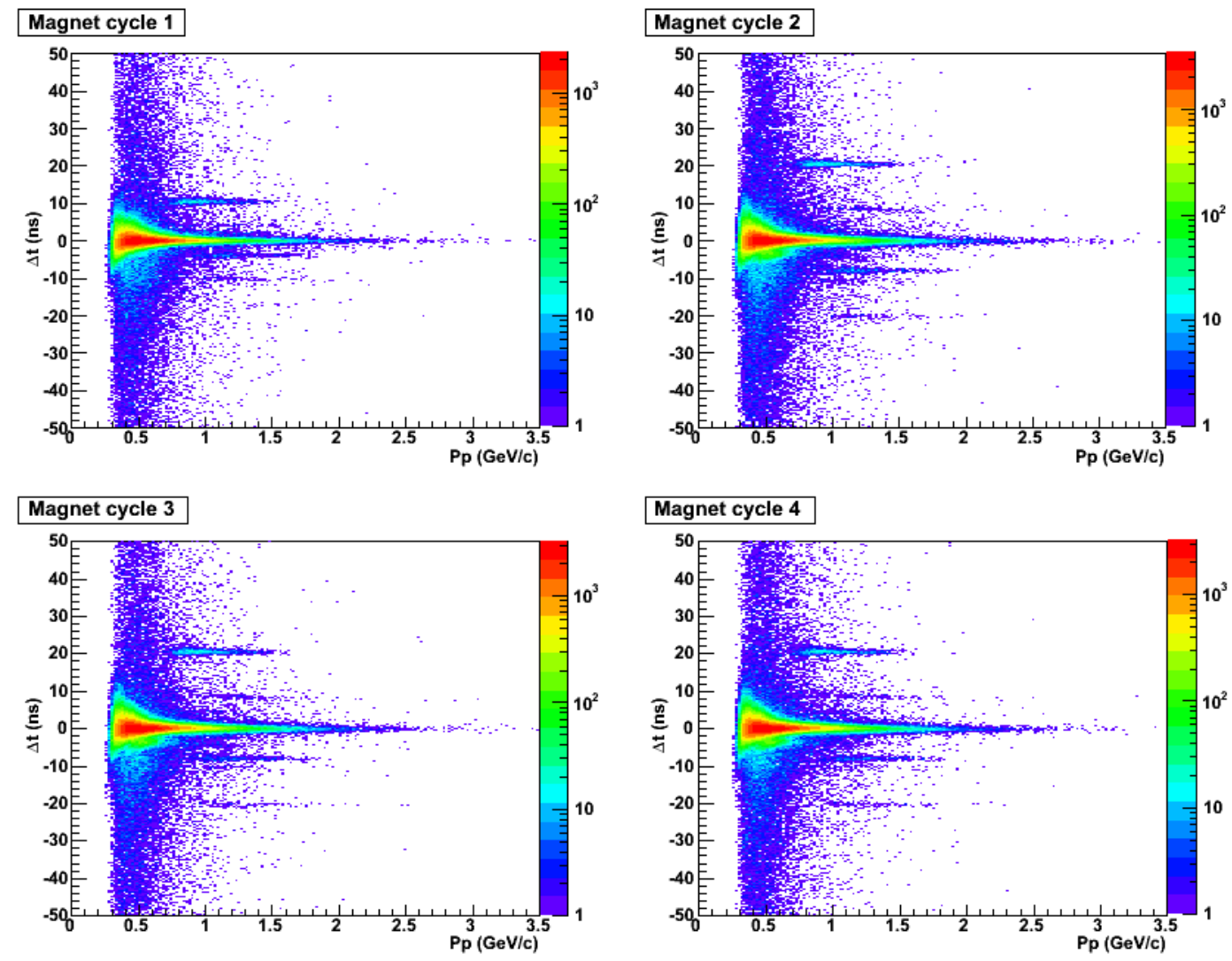

FIG. 58. $\Delta t$ distribution (uncorrected) as a function of proton momentum, $p_{p}$ for each magnet cycle. 


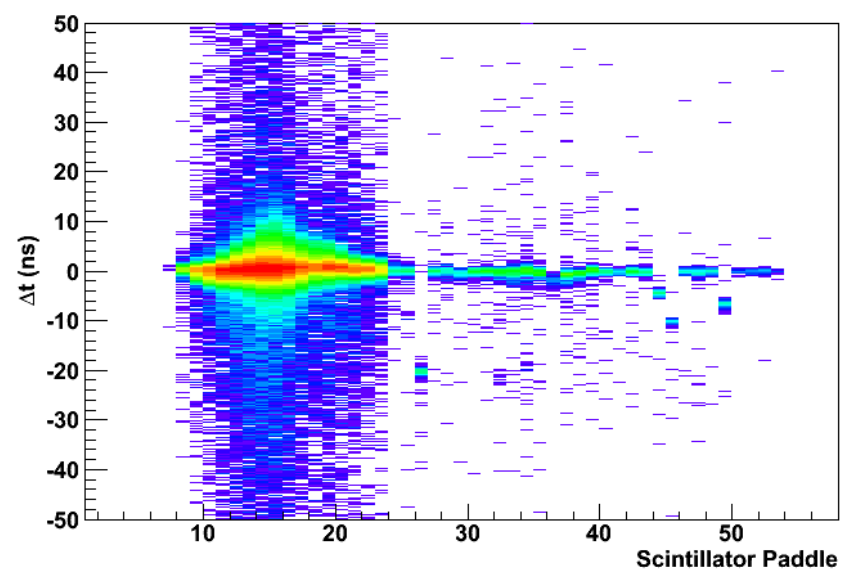

FIG. 59. Paddle by paddle $\Delta t$ distribution of sector 2 in magnet cycle 2,3 and 4 . Four scintillator paddles $(26,44,45,49)$ needed to be recalibrated.

In order to identify the scintillator paddles with offsets in each sector, the $\Delta t$ distribution of each paddle in each sector was studied individually. The $\Delta t$ distribution, paddle by paddle, for sector 2 is shown in Fig. 59. The $\Delta t$ distribution for each paddle was then fitted with a Gaussian to extract the offset constant, $T_{\text {offset }}$. The offset constants were identified for each paddle in each sector and used to correct $\Delta t$. Note that the $T_{\text {offset }}$ values for magnet cycle 1 were determined separately. Figure 60 shows the $\Delta t$ distribution as a function of proton momentum after the offset corrections. The band on the negative side of the $\Delta t$ distribution seen in $e^{+} p$ events corresponds to events with incorrectly assigned (swapped) positron and proton. Timing information was then used to correctly identify the positron and the proton as follows:

- Assume track 1 is the positron and track 2 is the proton (unswapped orientation). Apply elastic kinematic cuts and calculate $\Delta t$.

- Assume track 1 is the proton and track 2 is the positron (swapped orientation). Apply elastic kinematic cuts and calculate $\Delta t$.

- If the event passes the elastic kinematic cuts in both orientations, then choose the orientation that has the minimum $\Delta t$. 

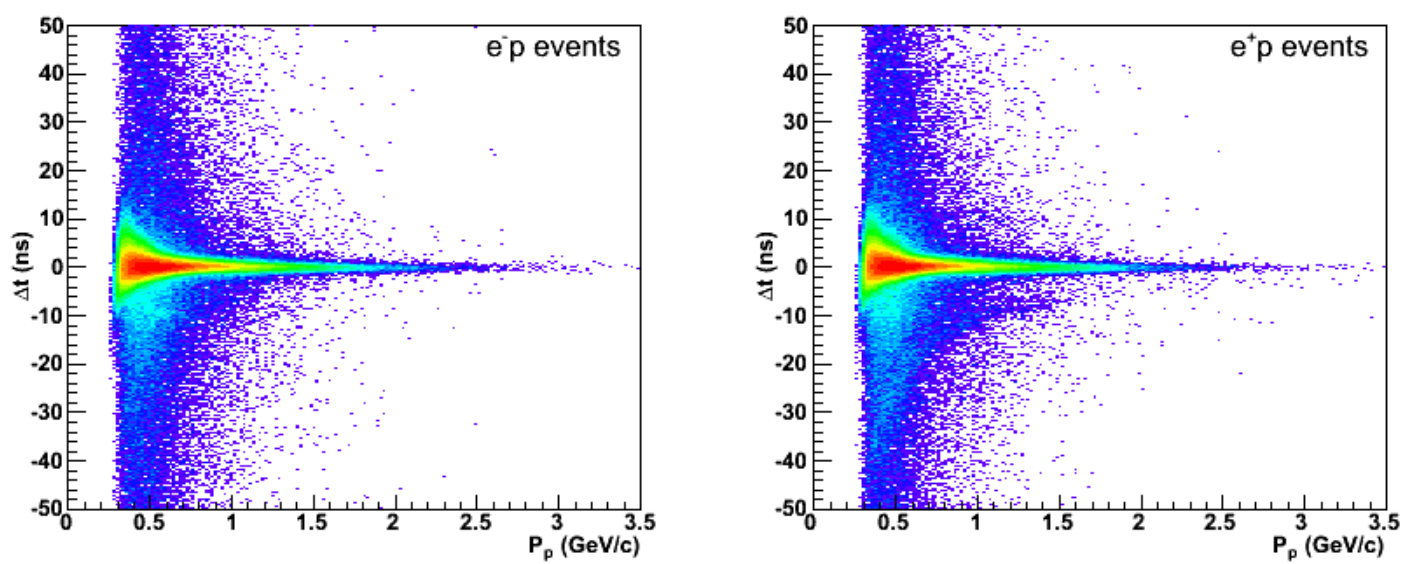

FIG. 60. Offset-corrected $\Delta t$ distribution as a function of proton momentum, $p_{p}$ for $e^{-} p$ events (right) and $e^{+} p$ events (left). The faint band on the negative side of the $\Delta t$ distribution seen in $e^{+} p$ events corresponds to events with incorrectly assigned (swapped) positron and proton. These events were corrected later.

\subsubsection{VERTEX CUT}

In order to remove any contamination from non-target material such as target entrance and exit windows, a cut is made on z-vertex $\left(v_{z}\right)$, the location along the beam line where the particle originated. Figure 61 shows the z-vertex measured for leptons. The TPE target was located $30 \mathrm{~cm}$ upstream of the CLAS center. Therefore a loose cut of $-44 \mathrm{~cm}<v z_{l}<-16 \mathrm{~cm}$ was first applied to the data. To ensure that the proton and the lepton came from the same interaction point, the difference between the z-vertex measured for each particle is calculated and used to make another vertex cut on TPE data. Figure 62 shows that the widths of the difference $\left(v z_{l}-v z_{p}\right)$, where $v z_{l}$ and $v z_{p}$ are the z-vertex of the lepton and proton, vary slightly with $\varepsilon$. The second vertex cuts were centered at the mean of the $v z_{l}-v z_{p}$ distribution with widths of 3.5 standard deviations to each side. 


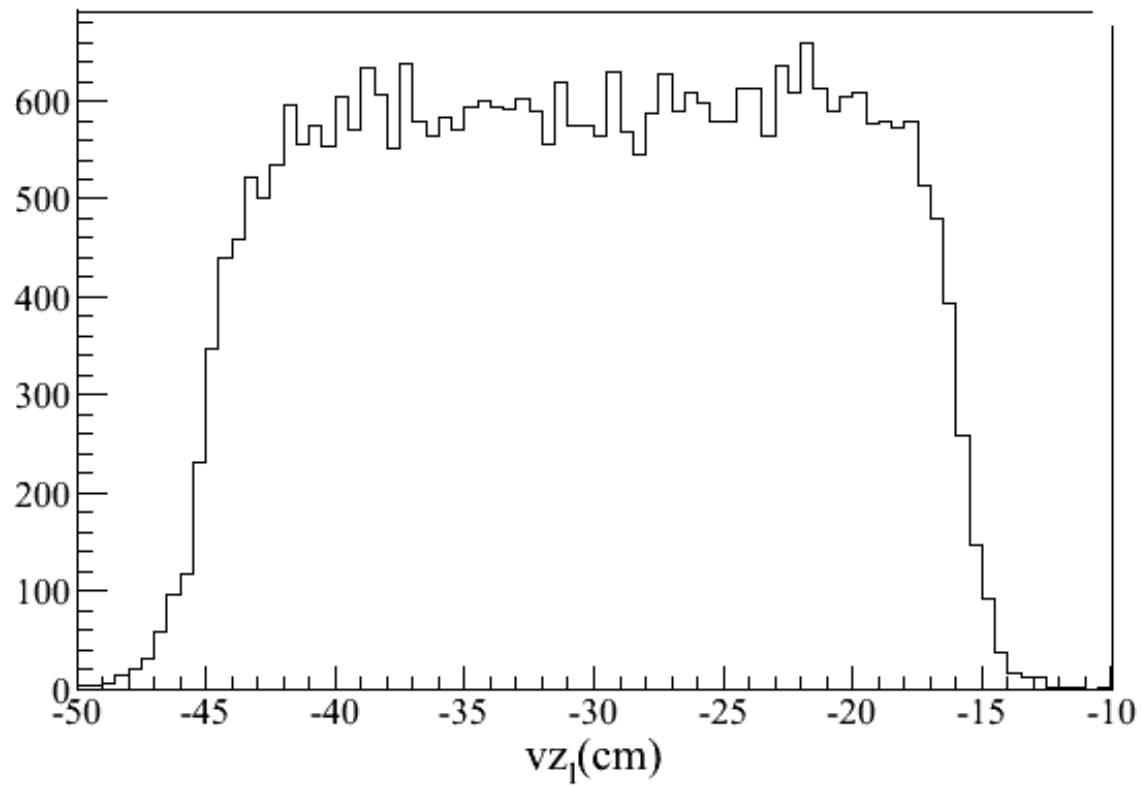

FIG. 61. Lepton vertex distribution $\left(v z_{l}\right)$.
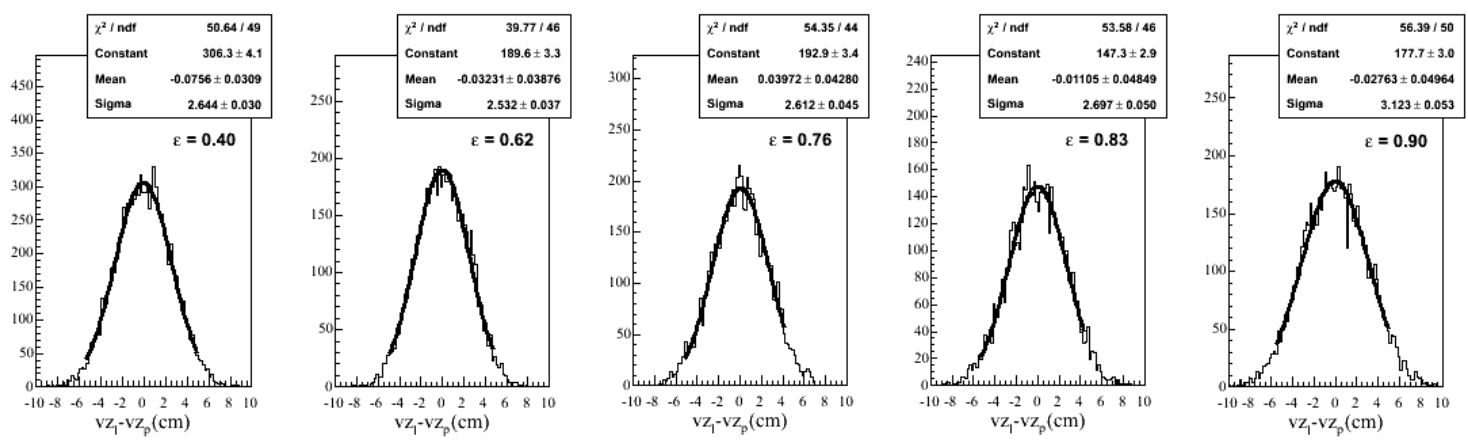

FIG. 62. The difference between the lepton vertex $\left(v z_{l}\right)$ and the proton vertex $\left(v z_{p}\right)$. 

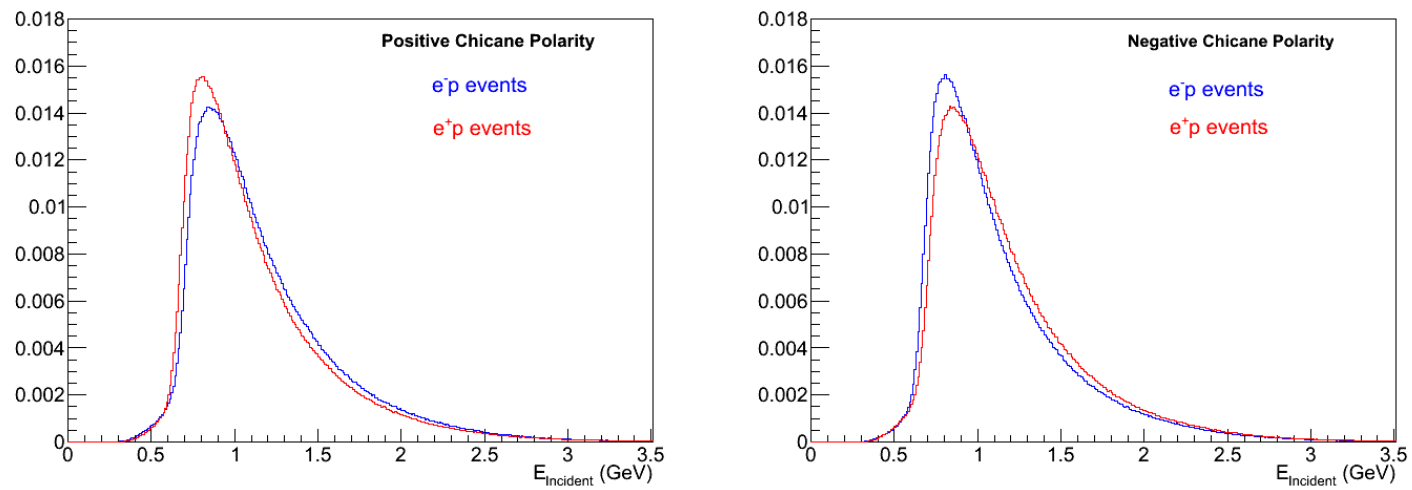

FIG. 63. Reconstructed incident energy distributions for elastic scattering events using scattering angles $\left(E_{\text {Incident }}^{1}\right)$ for positive (left) and negative (right) chicane magnet settings for $e^{-} p$ (blue) and $e^{+} p$ (red) events.

\subsubsection{INCIDENT BEAM ENERGY CUT}

Figure 63 shows the reconstructed incident energies of elastic $e^{-} p$ and $e^{+} p$ events using their scattering angles for both chicane polarities. The distributions are different for the two chicane polarities due to the asymmetry of the chicane. However, the incident energy distribution of $e^{-} p$ events at positive chicane polarity is similar to the incident energy distribution of $e^{+} p$ events at negative chicane polarity and vice versa. When we combined the data of the two chicane polarities, the incident energy distributions obtained for elastic $e^{-} p$ and $e^{+} p$ events are similar (see Fig. 64). We cut the incident energy at $0.85 \mathrm{GeV}$ to avoid the region where the beam energy is changing very rapidly and where any small asymmetries in the beam energy distribution will be greatly magnified in the elastic scattering cross section ratios.

\subsubsection{FIDUCIAL CUTS}

It is very important that the detector has similar acceptance for electrons and positrons to reduce the systematic uncertainties in the cross section ratio. Therefore fiducial cuts were applied to the reconstructed data to select the kinematic regions where both electrons and positrons were detected by CLAS for both torus settings and to avoid the regions of CLAS where the particle detection efficiency changes rapidly. 


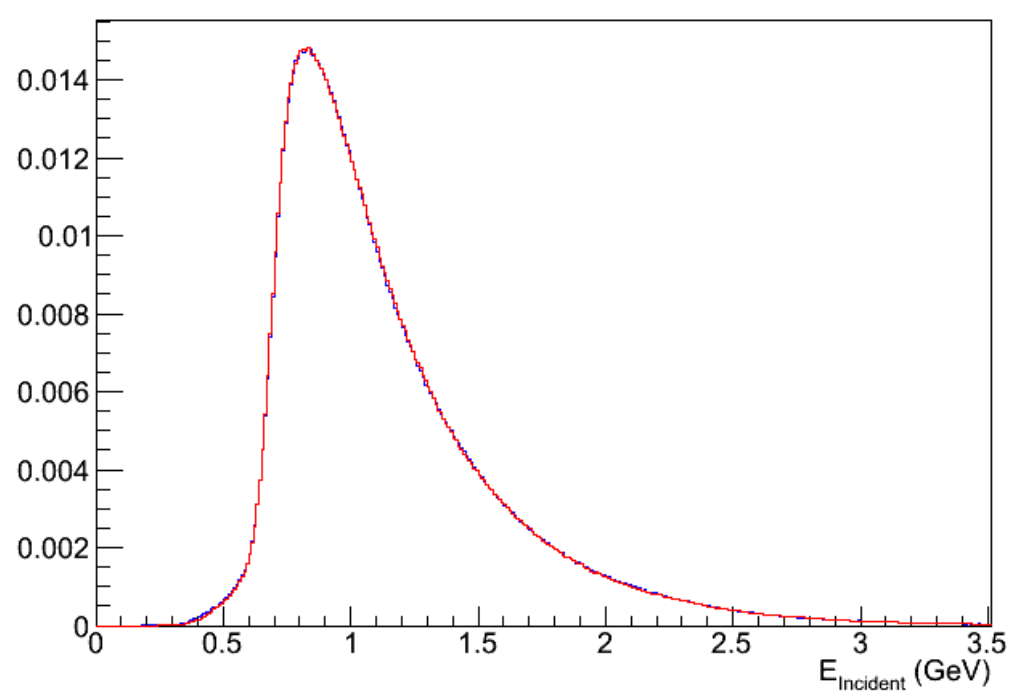

FIG. 64. Reconstructed incident beam energy distributions of elastic scattering events using scattering angles $\left(E_{\text {Incident }}^{1}\right)$ for $e^{-} p$ (blue) and $e^{+} p$ (red) events.

The fiducial cut regions were determined by studying the azimuthal angle $(\phi)$ and scattering angle $(\theta)$ distributions of reconstructed tracks in different momentum bins as shown in Fig. 65 and Fig. 66. The distributions were summed over all sectors. These cuts were first checked by studying electrons and positive pions for both positive and negative torus magnet settings in one-dimensional slices of $\theta$ as a function of $\phi$. Those studies showed that the acceptance for in-bending and out-bending tracks are significantly different. We used the functional forms of the fiducial cuts derived by the g13 collaboration, but the fit parameters were adjusted using TPE data.

Figure 65 shows that the angular acceptance of electrons in the positive torus magnetic fields have some momentum dependence at small $\theta$ because they are bent inwards by the torus magnet fields. Therefore, a momentum dependent $\theta$-shift was introduced to the in-bending tracks as $\theta^{\prime}=\theta+\theta$-shift where

$$
\theta \text {-shift }=0.1913+\frac{0.2012 \mathrm{GeV}}{(p+0.2997 \mathrm{GeV})} \mathrm{rad}
$$

This value of $\theta^{\prime}$ is then used in Eqs.(58) and (59). See Fig. 67. The fiducial cuts used for in-bending and out-bending tracks are $|\phi|<\phi_{\max }$ where, for in-bending tracks 

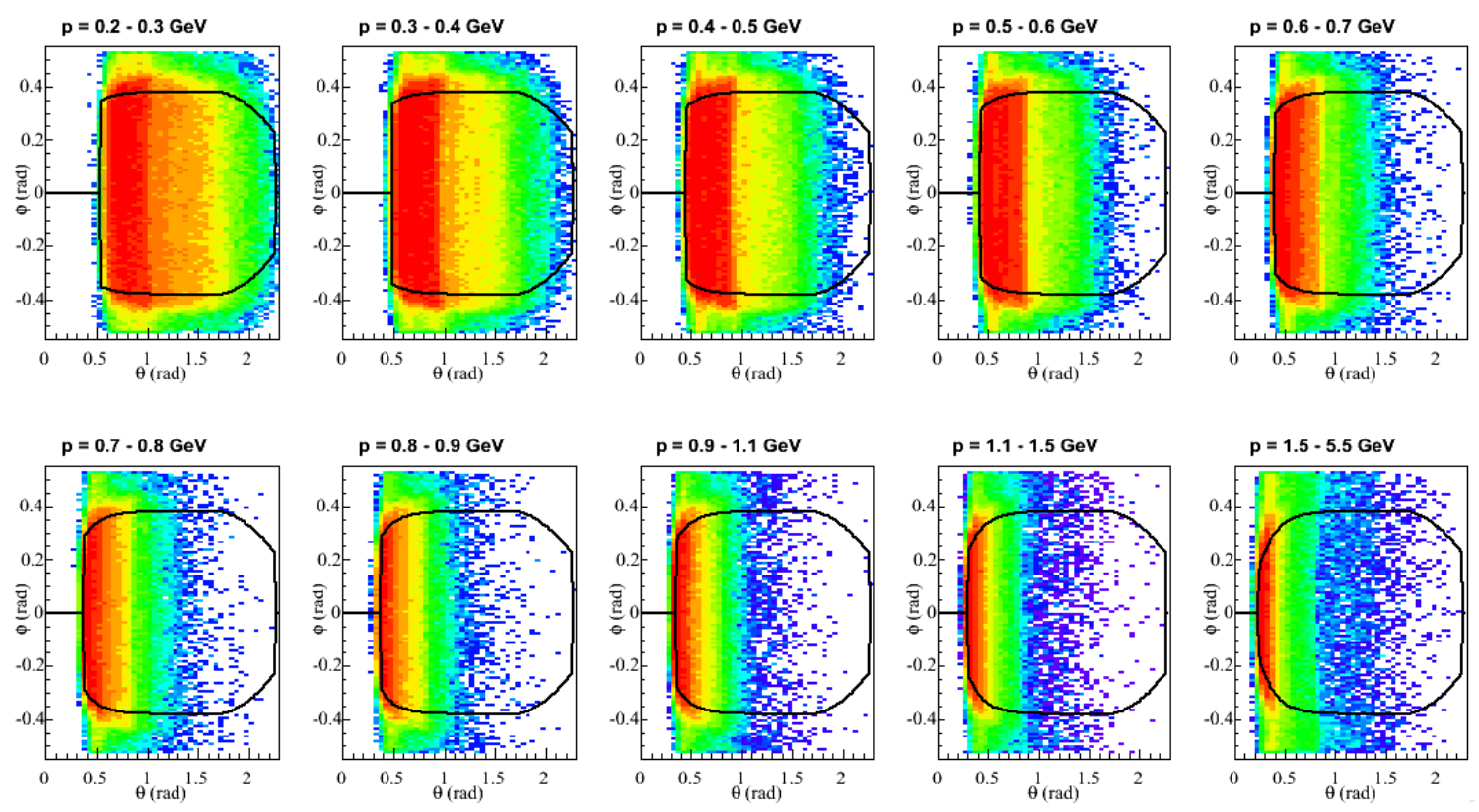

FIG. 65. The number of reconstructed electrons as a function of $\theta$ and $\phi$ for positive torus magnet setting (in-bending tracks) for all sectors for different momentum bins. Fiducial cuts are drawn in black.

(with $\phi_{\max }$ in radians),

$$
\phi_{\max }=\left\{\begin{array}{lc}
0.38\left(1-e^{-6.5 \theta^{\prime}+1.0}\right), & 0.237<\theta^{\prime}<1.7 \mathrm{rad} \\
-0.4 \theta^{\prime 2}+1.07 \theta^{\prime}-0.674, & \theta^{\prime}>1.7 \mathrm{rad}
\end{array}\right\}
$$

and for out-bending tracks,

$$
\phi_{\max }=\left\{\begin{array}{lc}
0.39\left(1-e^{-6.5 \theta^{\prime}+0.6}\right), & 0.187<\theta^{\prime}<1.92 \mathrm{rad} \\
-0.44 \theta^{\prime 2}+1.07 \theta^{\prime}-0.042384, & \theta^{\prime}>1.92 \mathrm{rad}
\end{array}\right\}
$$

Figure 65 and 66 show that the fiducial cuts are within the regions of uniform acceptance for both in-bending and out-bending leptons. Both fiducial cuts were applied to all leptons.

\subsubsection{DEAD DETECTOR CUTS}

Another important part of the data analysis is to identify the inefficient detector channels which involve dead, broken and/or inefficient detector components and remove the events that hit those channels. These channels can have a large impact on 

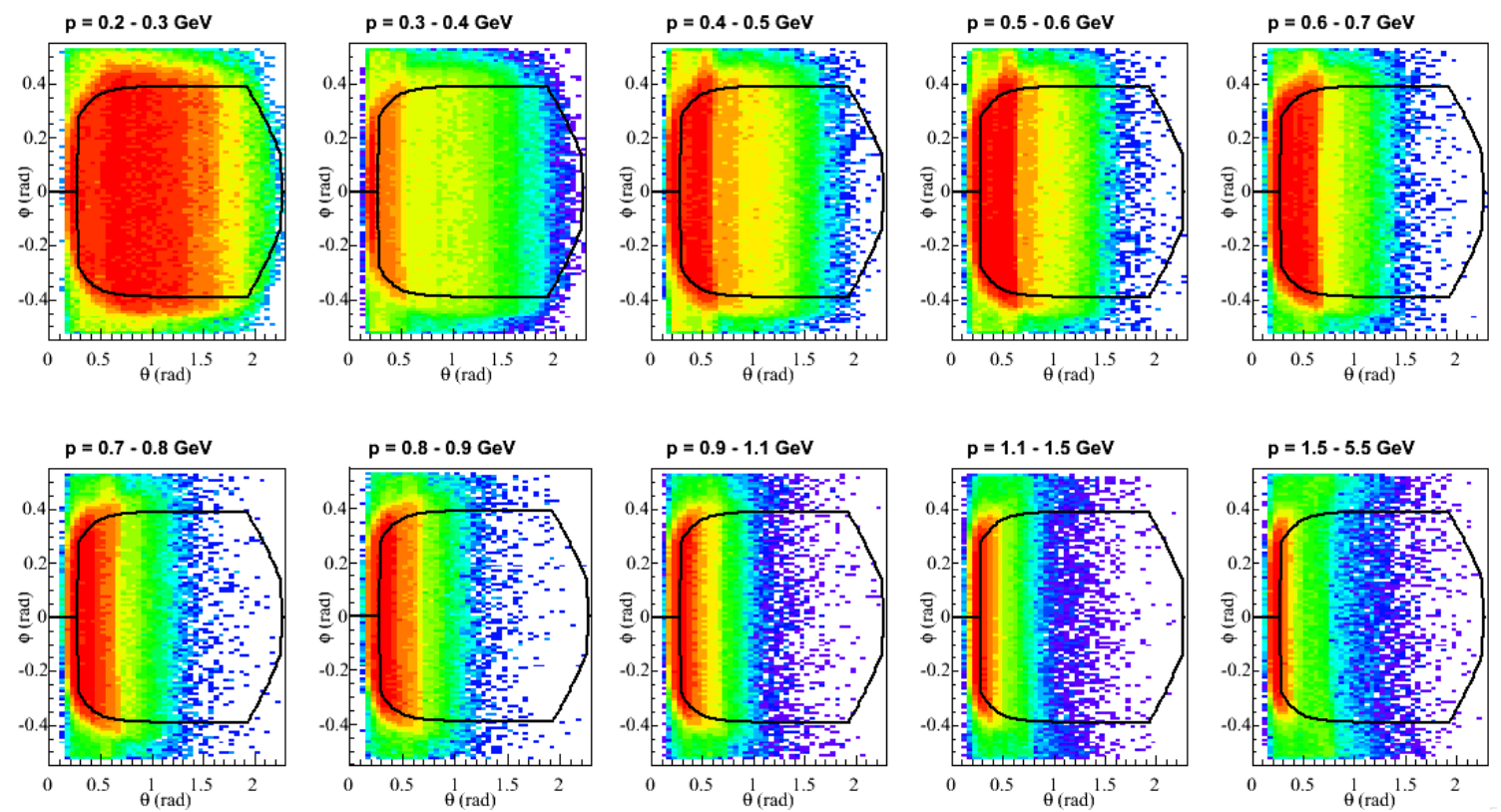

FIG. 66. The number of reconstructed electrons as a function of $\theta$ and $\phi$ for negative torus magnet setting (out-bending tracks) for all sectors for different momentum bins. Fiducial cuts are drawn in black.

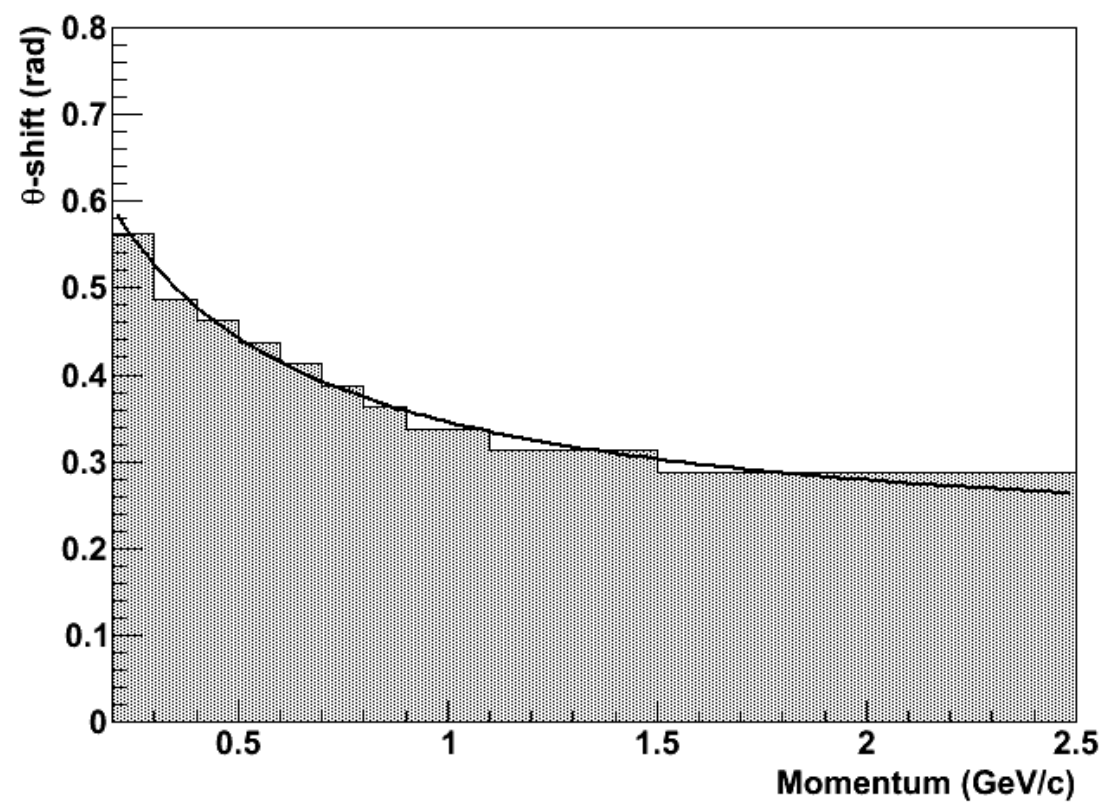

FIG. 67. $\theta$-shift vs. momentum of reconstructed electrons for positive torus magnet setting (in-bending tracks). The fit to this distribution was used for determining the shift of fiducial cuts for in-bending tracks. 


\begin{tabular}{|c|c|}
\hline Sector & Bad TOF paddles \\
\hline 1 & 34 \\
2 & 26 \\
3 & 16,23 \\
4 & $16,23,29$ \\
5 & $8,12,23,30$ \\
6 & 29 \\
\hline
\end{tabular}

TABLE 3. List of bad TOF paddles.

the event selection process and could produce a significant asymmetry in the final cross section ratio.

Several bad TOF scintillator paddles were found during the timing correction study and those are listed in Table 3. Those bad TOF paddles were either dead or noisy and therefore needed to be removed from the TPE dataset.

The sector 3 drift chamber and EC had many dead regions compared to other sectors. In order to reduce the systematic bias due to these regions, any events with either lepton or proton detected in sector 3 with $\theta<45^{\circ}$ were discarded.

The TPE experiment crucially needs similar detector acceptances for both electrons and positrons. But the inefficient detector removal mentioned above can bias the lepton efficiencies. In order to take those biases into account, a special CLAS algorithm called "swimming" was used. This algorithm calculates the trajectory of particles through the CLAS detector system and determines the hit positions on the detector planes. For each event, it generates a conjugate lepton with the same momentum and angles and swims the conjugate lepton through CLAS. For example, if it is an $e^{-} p$ event, the algorithm generates an $e^{+}$with the same momentum and angles and swims it through the CLAS. If this conjugate particle hits a bad TOF paddle or falls outside the fiducial cuts, the original event is discarded.

\subsubsection{BACKGROUND SUBTRACTION}

Figure 68 shows that there is a significant background under the elastic event peak even after applying elastic kinematic cuts and other required cuts, especially at 

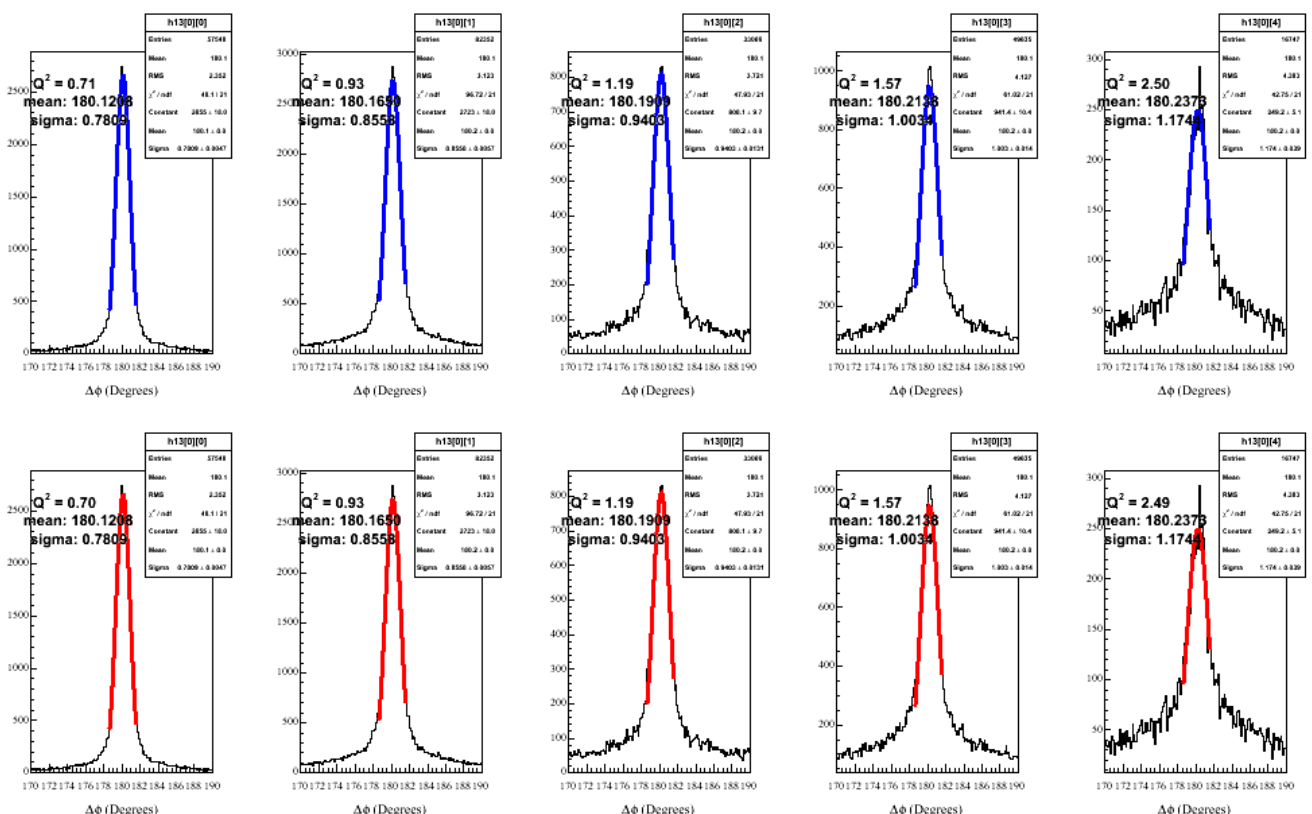

FIG. 68. $\Delta \phi$ distributions for different $Q^{2}$ bins for $0.1<\varepsilon<0.5$ after other elastic cuts, top row for $e^{-} p$ events and bottom row for $e^{+} p$ events.

low $\varepsilon$ and high $Q^{2}$. To obtain accurate $e^{ \pm} p$ cross-section ratios, it is very important to determine this background correctly and to subtract it from the signal. Further studies showed that the background is symmetrical for the $\Delta \phi$ distributions but not for $\Delta p_{p}$ and $\Delta E^{ \pm}$. Therefore, $\Delta \phi$ distributions were used to determine the background.

The data was divided into bins in $Q^{2}$ and $\varepsilon . \Delta \phi$ distributions were made for each bin, for $e^{-} p$ and $e^{+} p$ events separately. The tails of the $\Delta \phi$ distributions were fitted with different functions; linear, polynomial and Gaussian. Considering the $\chi^{2} /$ d.f and overall goodness of all fits, the Gaussian fits best described the backgrounds. We also used a sampling method to determine the background at low $\varepsilon$. In the sampling method, the events on the tails of the $\Delta E^{-}$distributions were sampled and scaled to fit the tails of the $\Delta \phi$ distributions. Figure 69 shows the $\Delta E^{-}$and $\Delta \phi$ distributions for the worst case of background at low $\varepsilon$ and high $Q^{2}$. The sampled and fitted backgrounds agree well in this bin. At high $\varepsilon$ where the background is small, the sampling method fails because the $\Delta E^{-}$peak becomes too wide. Therefore only the fitting method was used as to subtract background in this analysis. 

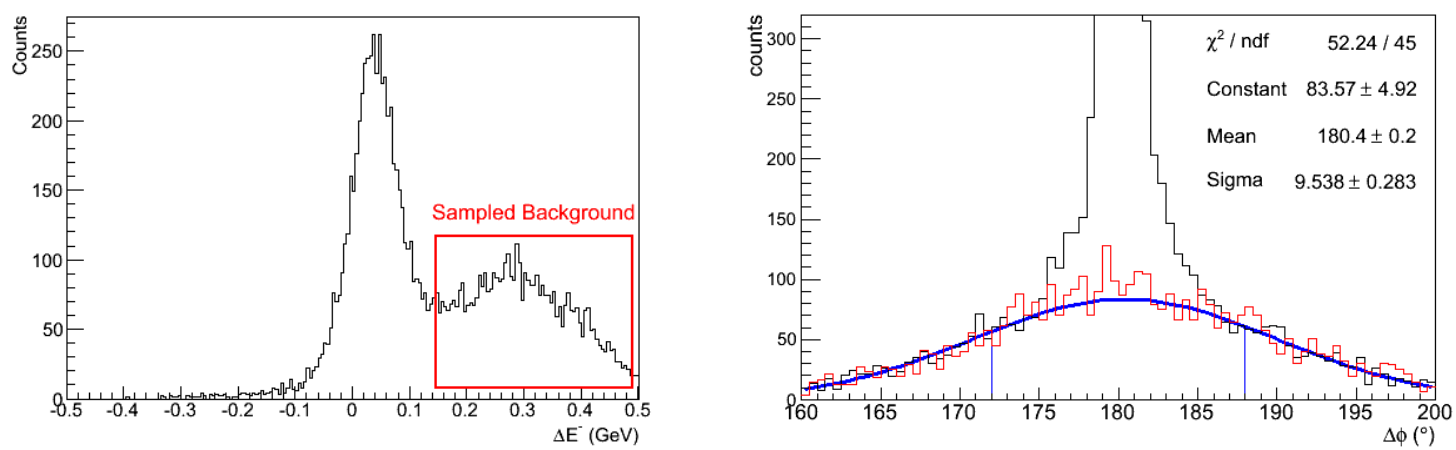

FIG. 69. $\Delta E^{-}$(left) and $\Delta \phi$ (right) distributions at $\varepsilon=(0.2,0.5)$ and $Q^{2}=(1.0,4.0)$. $\Delta \phi$ distribution shows both sampling (red histogram) and fitting (blue curve) backgrounds.
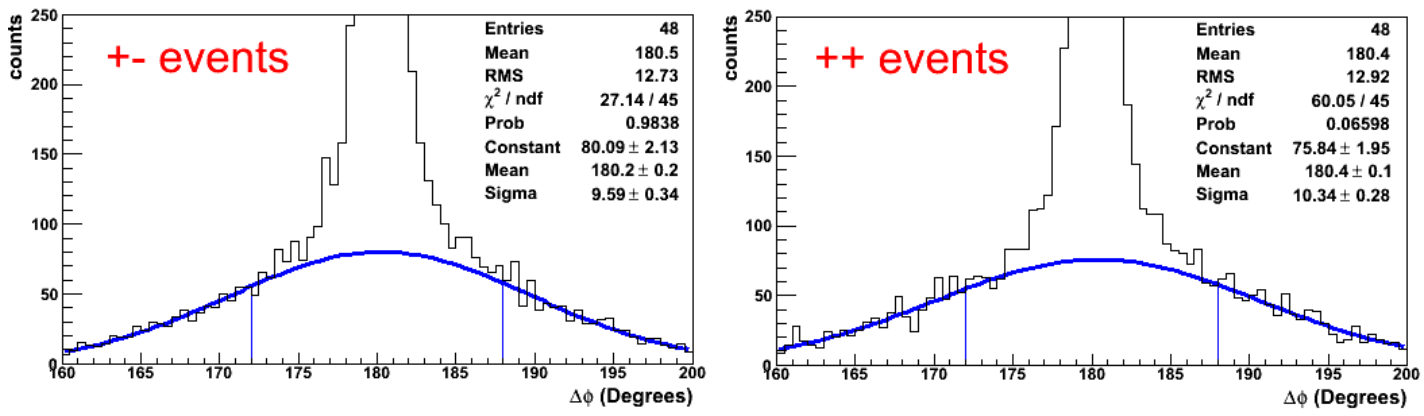

FIG. 70. $\Delta \phi$ distributions with Gaussian fits to the backgrounds for $e^{-} p$ (left) and $e^{+} p$ (right) events for data at $\varepsilon=(0.2,0.5)$ and $Q^{2}=(1.0,4.0)$. All other cuts and corrections were applied except $\Delta \phi$ cuts. 
Figure 70 shows the Gaussian functions fitted to the $\Delta \phi$ background for the worst case $\varepsilon=(0.2,0.5)$ and $Q^{2}=(1.0,4.0)$. The backgrounds for $e^{-} p$ and $e^{+} p$ are similar. The magnitude of the fit background depends slightly on the fitting range. We compared three different fitting ranges: $\left(160^{\circ}-172^{\circ}: 188^{\circ}-200^{\circ}\right)$, $\left(160^{\circ}-170^{\circ}: 190^{\circ}-200^{\circ}\right)$ and $\left(160^{\circ}-174^{\circ}: 186^{\circ}-200^{\circ}\right)$. The variation of the results with the fitting range is discussed in detail under systematic uncertainties. 


\section{CHAPTER 5}

\section{RESULTS}

\subsection{KINEMATIC COVERAGE AND BIN SELECTION}

The $Q^{2}$ vs. $\varepsilon$ distributions of the elastic scattering $e^{-} p$ and $e^{+} p$ events for both torus magnetic settings are shown in Fig. 71, including elastic kinematic cuts, corrections and other cuts except background subtraction. The wide $Q^{2}-\varepsilon$ coverage of TPE data can be clearly seen in all four cases. However, there is a lack of data around $\varepsilon=0.7$ at low $Q^{2}$ which is due to the primary trigger used in the experiment. To record an event, there should be one particle track that hits the forward Electromagnetic Calorimeters (EC) and TOF and a second particle that hits the TOF in the opposite sector. In the positive torus magnet setting, the positive charged particles are out-bending and therefore are more likely to both miss the EC.

The elastic scattering data was binned to study the $Q^{2}$ and $\varepsilon$ dependence of the cross section ratios (see Fig. 72). These bins were selected to have similar statistical significance. The bin boundaries were adjusted to avoid the trigger hole and to obtain approximately similar average $Q^{2}$ or average $\varepsilon$ for all bins. Note that the edges of the distributions were also avoided, since the acceptance for in-bending and out-bending particles varies rapidly at the edges.

Further analysis and the charge asymmetry ratios for the five high $Q^{2}$ data bins and the five low $\varepsilon$ data bins will be discussed in next section. The remaining data is the subject of Dipak Rimal's PhD thesis [39].

\subsection{CHARGE ASYMMETRY RATIO}

The elastic scattering cross section can be written as

$$
\sigma=\sigma_{m o t t}\left(\frac{1}{1+\tau}\right)\left[G_{E}^{2}\left(Q^{2}\right)+\frac{\tau}{\varepsilon} G_{M}^{2}\left(Q^{2}\right)\right]
$$

and it can be directly measured by

$$
\sigma \propto \frac{N}{A \mathcal{L} f_{\mathrm{rad}}}
$$



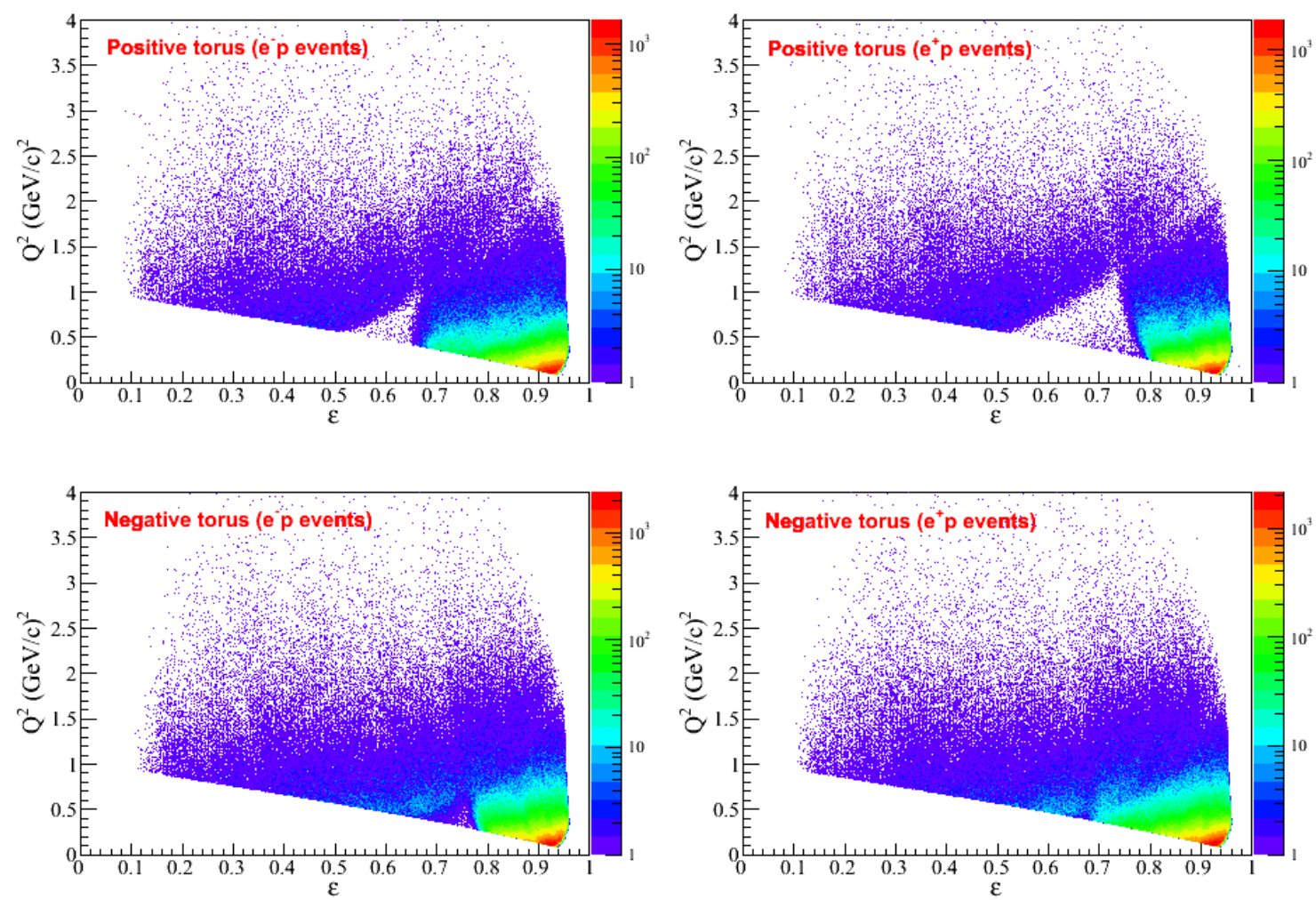

FIG. 71. The $Q^{2}$ vs. $\varepsilon$ distributions for the $e^{-} p$ and $e^{+} p$ elastic scattering events for both torus magnetic settings.
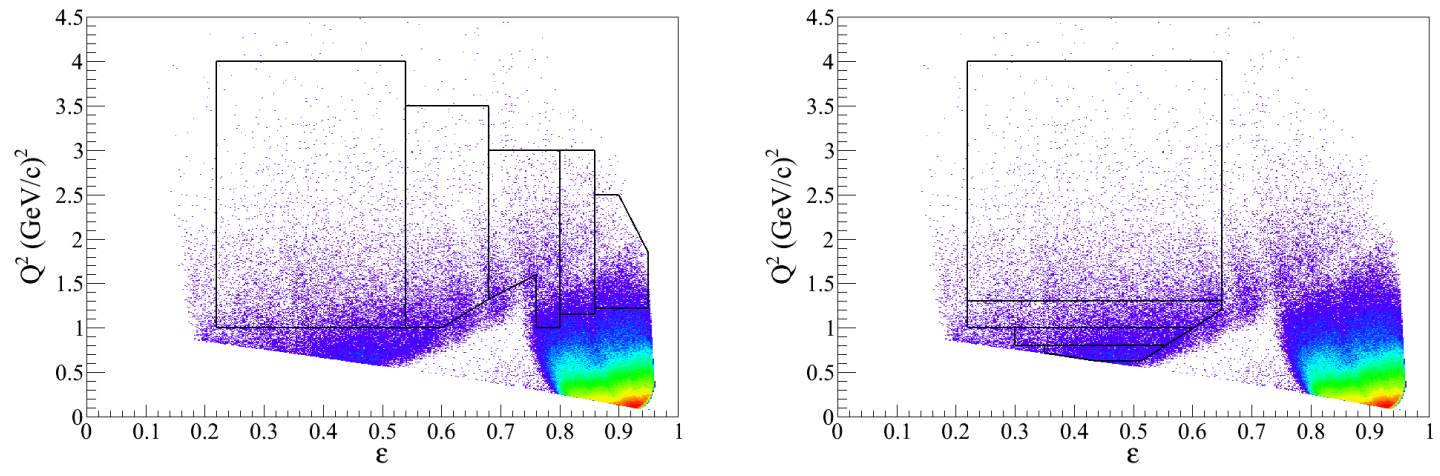

FIG. 72. The bin boundaries for high $Q^{2}$ data (left) and low $\varepsilon$ data (right). 
where $N$ is the number of elastic scattering events, $A$ is the corrected acceptance, $\mathcal{L}$ is the luminosity, and $f_{\text {rad }}$ is the radiative correction. The acceptance difference of $e^{+} p$ and $e^{-} p$ scattering data was carefully studied and minimized by applying the fiducial cuts, the dead detector cuts and the swimming procedure discussed in the previous section. The luminosity difference of the $e^{ \pm} p$ scattering events is controlled by reversing the chicane magnetic setting and will be discussed and taken into account. The charge-symmetric part of the radiative correction cancels in the cross section ratio. Calculation of charge-asymmetric part of the radiative correction to the cross section ratio is discussed in Section 5.5. In this analysis, the ratio between the number of $e^{+} p$ and $e^{-} p$ elastic scattering events is calculated in three steps as discussed below.

1. Single ratio $\left(R_{1}\right)$

The single ratios are calculated for each magnet configuration as follows. Here \pm \pm represents the torus magnet polarity and chicane magnet polarity, respectively. The proton acceptance differences of $e^{ \pm} p$ scattering events will cancel out in the single ratio,

$$
R_{1}^{ \pm \pm}=\frac{N_{e^{+} p}^{ \pm \pm}}{N_{e^{-} p}^{ \pm \pm}}
$$

For example, $R_{1}^{++}$is the ratio for positive torus and positive chicane.

\section{Double ratio $\left(R_{2}\right)$}

The single ratios for different torus polarities are then combined to form a double ratio for a given chicane polarity. The $\pm \operatorname{sign}$ in $R_{2}$ represents the chicane magnet polarity. The remaining lepton acceptance will cancel in the double ratio,

$$
R_{2}^{ \pm}=\sqrt{R_{1}^{- \pm} R_{1}^{+ \pm}}
$$

3. Quadruple ratio $(R)$

Finally, the double ratios for different chicane magnet polarities are combined together to form a quadruple ratio as shown below. If there is any luminosity difference due to the beam transport asymmetry, it will cancel out in the quadruple ratio,

$$
R=\sqrt{R_{2}^{-} R_{2}^{+}}
$$

These three ratios will be calculated for each kinematic bin and the quadruple ratios will be radiatively corrected to form the final charge asymmetry ratios. 


\begin{tabular}{cccccc}
\hline \hline \multirow{2}{*}{ Bin } & \multicolumn{2}{c}{$Q^{2}\left(\mathrm{GeV}^{2}\right)$} & & \multicolumn{2}{c}{$\varepsilon$} \\
\cline { 2 - 3 } \cline { 5 - 6 } & Range & Bin center & & Range & Bin center \\
\hline 1 & $1.00-4.00$ & 1.44 & & $0.22-0.54$ & 0.40 \\
2 & $1.00-3.50$ & 1.45 & & $0.54-0.68$ & 0.60 \\
3 & $1.00-3.00$ & 1.46 & & $0.68-0.80$ & 0.76 \\
4 & $1.13-3.00$ & 1.47 & & $0.80-0.86$ & 0.83 \\
5 & $1.21-2.50$ & 1.47 & & $0.86-0.95$ & 0.90 \\
\hline \hline
\end{tabular}

TABLE 4. Bin ranges and bin centers for $Q^{2}$ and $\varepsilon$ for high $Q^{2}$ data.

\subsection{RESULTS}

\subsubsection{HIGH $Q^{2}$ DATA}

As seen in Fig. 72, the $Q^{2}>1 \mathrm{GeV}^{2}$ data are binned into five bins to study the $\varepsilon$ dependence of the cross section ratio. The weighted average $Q^{2}$ of each bin is $\sim 1.45$ $\mathrm{GeV}^{2}$. The bin ranges and centers for each bin are summarized in Table 4. The elastic scattering $e^{ \pm} p$ events were carefully selected using all kinematic and other cuts. The background $e^{ \pm} p$ events were also subtracted and the single, double and quadruple ratios were calculated for all bins.

Figure 73 shows the double ratios for the two chicane magnet polarities and the quadruple ratios for each high $Q^{2}$ kinematic bin. The double ratios for positive and negative chicane magnet setting are clearly different due to the $e^{ \pm}$transport asymmetries through the chicane. These asymmetries cancel in the quadruple ratio. More details of the beam asymmetry will be discussed under systematic uncertainties. The quadruple ratios at $Q^{2} \approx 1.45 \mathrm{GeV}^{2}$ increase with decreasing $\varepsilon$.

\subsubsection{LOW $\varepsilon$ DATA}

The $\varepsilon<0.7$ data are binned into four kinematic bins and the weighted average $\varepsilon$ of those bins is $\sim 0.45$ (see Fig. 72 and Table 5 ). These bins were used to measure the $Q^{2}$ dependence of the cross section ratio at low $\varepsilon$. The double ratios for different chicane magnet polarities and the quadruple ratios obtained for these kinematic bins 

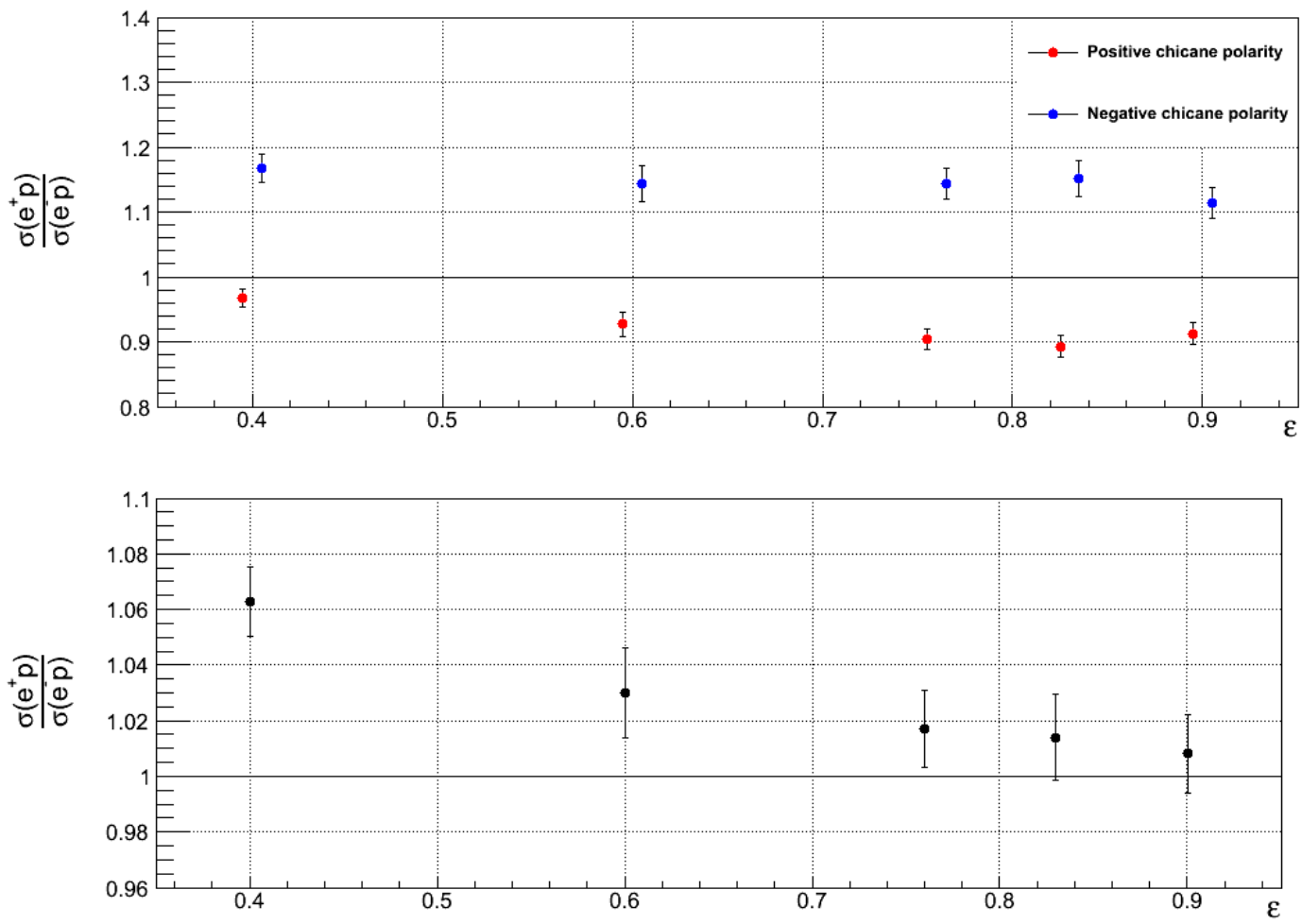

FIG. 73. Double ratios for positive and negative chicane polarities (top) and the quadruple ratio (bottom) as a function $\varepsilon$ at $Q^{2} \sim 1.45 \mathrm{GeV}^{2}$. No radiative corrections have been applied.

are shown in Fig. 74. The double ratios for different chicane polarities were different for all bins except at $Q^{2}=0.7 \mathrm{GeV}^{2}$. The quadruple ratios do not vary significantly with $Q^{2}$ at $\varepsilon \sim 0.45$. Further details of the quadruple ratios are discussed under systematic uncertainties in Section 5.4.

\subsection{SYSTEMATIC UNCERTAINTY}

The purpose of the TPE experiment is to measure the positron-proton to electronproton elastic scattering cross section ratios precisely. There were several techniques used to control the systematic uncertainties. The remaining sources of uncertainty are discussed below.

1. Differences in the electron and positron luminosities 


\begin{tabular}{cccccc}
\hline \hline \multirow{2}{*}{ Bin } & \multicolumn{2}{c}{$Q^{2}\left(\mathrm{GeV}^{2}\right)$} & & \multicolumn{2}{c}{$\varepsilon$} \\
\cline { 2 - 3 } \cline { 5 - 6 } & Range & Bin center & & Range & Bin center \\
\hline 1 & $0.63-0.80$ & 0.72 & & $0.35-0.55$ & 0.45 \\
2 & $0.80-1.00$ & 0.89 & & $0.30-0.60$ & 0.45 \\
3 & $1.00-1.30$ & 1.14 & & $0.22-0.65$ & 0.45 \\
4 & $1.30-4.00$ & 1.73 & & $0.22-0.65$ & 0.45 \\
\hline \hline
\end{tabular}

TABLE 5. Bin ranges and bin centers for $Q^{2}$ and $\varepsilon$ for low $\varepsilon$ data.
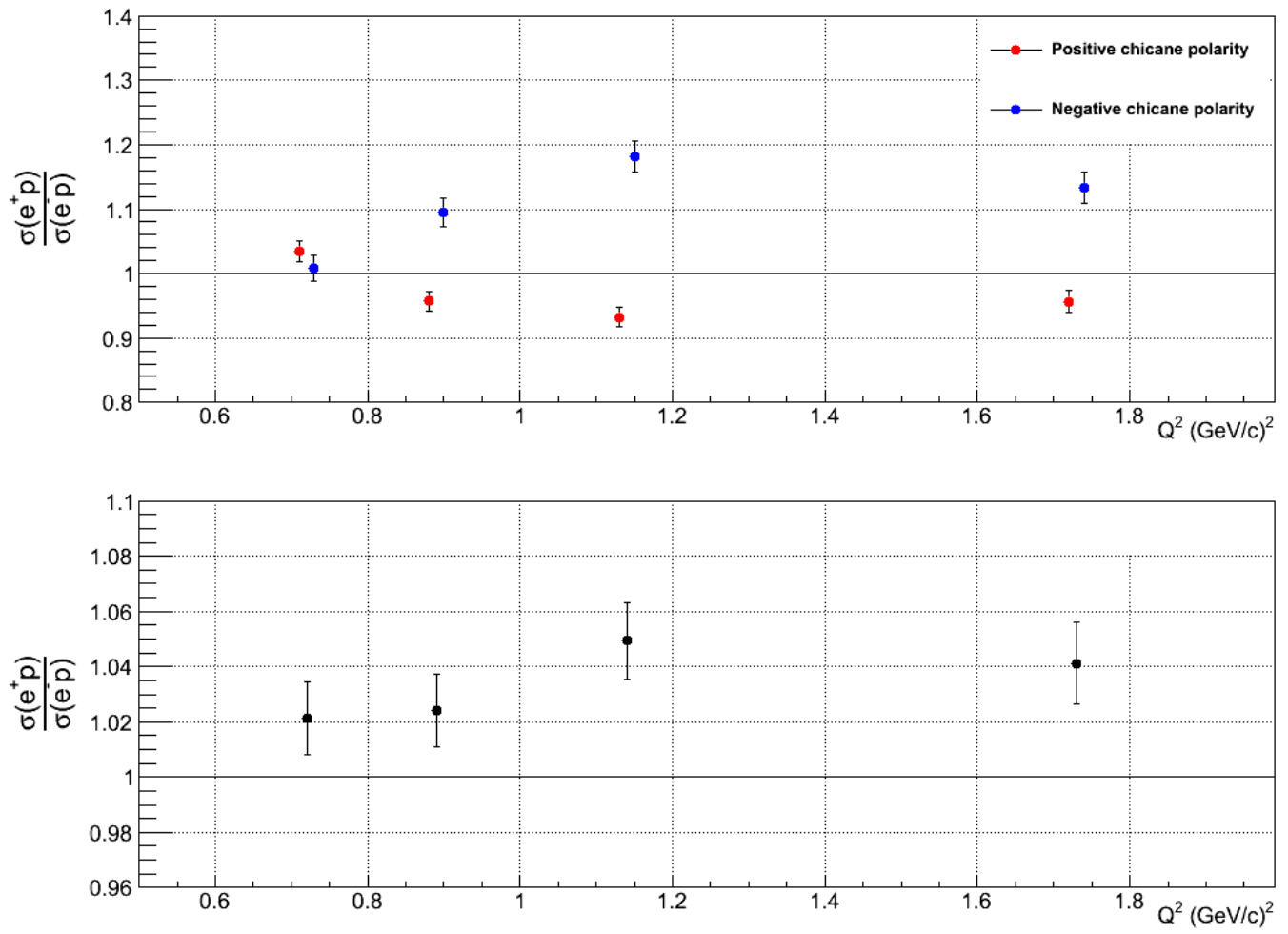

FIG. 74. Double ratios for positive and negative chicane polarities (top) and the quadruple ratio (bottom) as a function of $Q^{2}$ at $\varepsilon \sim 0.45$. No radiative corrections have been applied.

Electron-positron pair production is inherently charge symmetric and therefore 

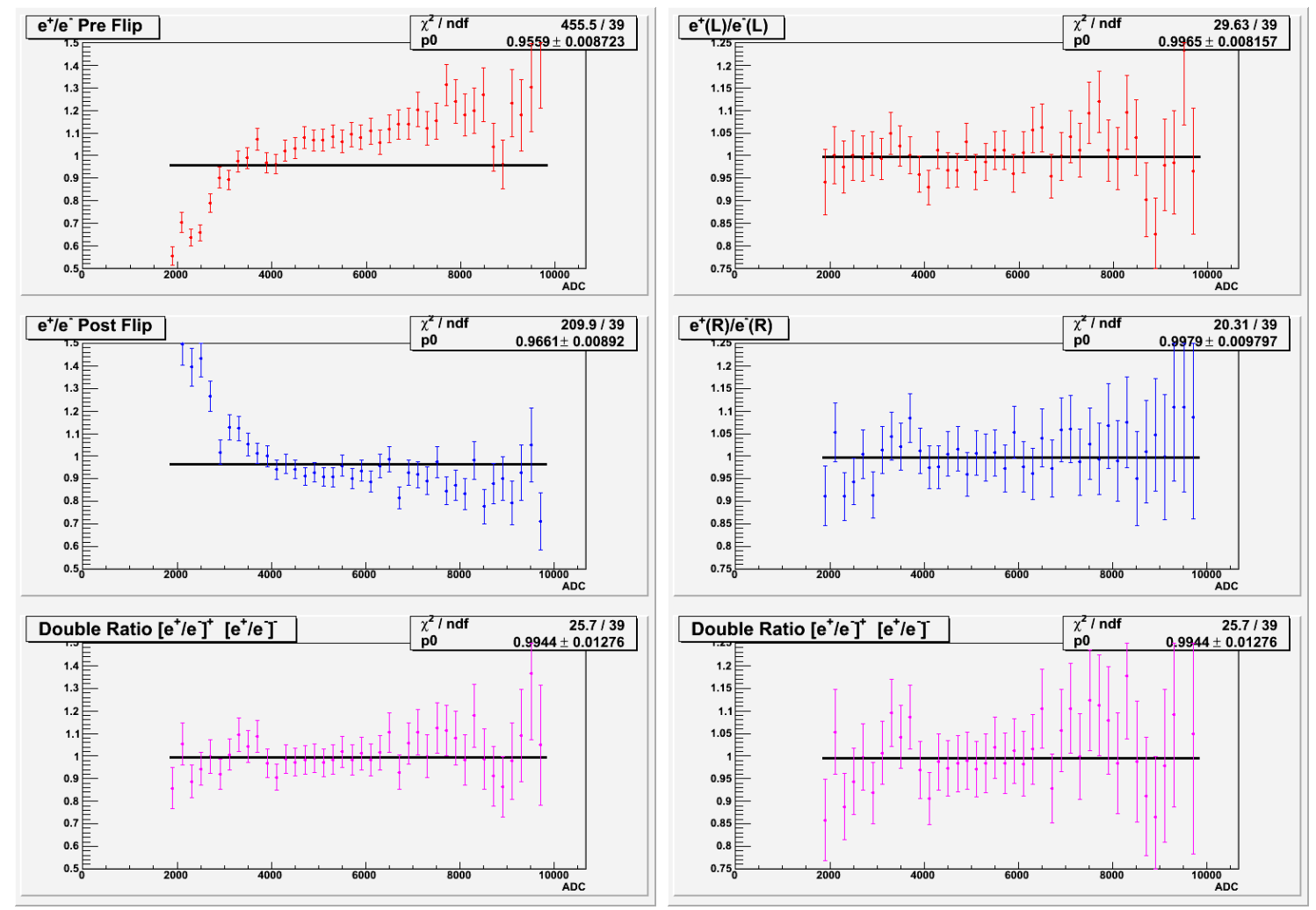

FIG. 75. The ratio of the incident positron energy density to the incident electron energy density as a function of incident energy (measured in channels where channel 1000 corresponds approximately to $370 \mathrm{MeV}$ ) for (left top panel) leptons passing on the chicane before the Jan 23 chicane flip, (left middle panel) and after the Jan 23 chicane flip, (right top panel) the same ratio for leptons passing on the left side of the chicane, (right middle panel) and the right side of the chicane, and (bottom of each panel) the product of the two ratios. The vertical scale is normalized to unity. The bottom right figure is identical to the bottom left figure, but with an expanded scale.

the electron and positron beam fluxes should be identical. In the experiment, the only differences in the two beams could come from differences in beam transport from the converter to the target. The chicane magnet fields were reversed four times during the run period to minimize the effects of these differences. The electron and positron incident energy distributions were measured with the TPE calorimeter. 
The ratios of the beam energy distributions are shown in Fig. 75. The left panel shows the ratio of positrons to electrons for leptons passing through the chicane before and after the January 23 chicane flip. The ratios are energy dependent and not consistent before and after the chicane flip. This indicates an asymmetry in beam transport. The right panel shows the same ratio for leptons passing on the right side of the chicane and on the left side of the chicane and demonstrates that the distribution of positrons passing through one side of the chicane is the same as the electrons passing through the same side even if the chicane is not symmetric. The product of the two single ratios is shown in the bottom panels and shows that the asymmetries in the beam transport cancel out in the double ratios.

The primary uncertainty due to the incident lepton flux comes from the variation in the chicane as its polarity was repeatedly flipped, as determined by the variance of the $e^{+} p / e^{-} p$ ratios for the different chicane cycles.

Figure 76 shows the double ratios obtained for different magnet cycles at positive and negative chicane magnet settings for bins at $\varepsilon=0.88$. The ratios are consistent and indicate that the chicane is reproducible at each flip. The ratios for magnet cycle 2 negative chicane are a few percent lower than the other cycles. This was also seen in the TPE calorimeter measurements from the January 14 chicane flip (see Fig. 77). The cycle 2 negative chicane data are therefore discarded from further analysis.

The total uncertainty $\left(\sigma_{\text {total }}\right)$ due to the magnet cycle dependence for each chicane setting is calculated as below from the variance of the measurements:

$$
\sigma_{\text {total }}^{2}=\frac{1}{n-1}\left\{\frac{\sum_{i=1}^{n}\left\{\frac{R_{i}-R_{\text {average }}}{\sigma_{i}}\right\}^{2}}{\sum_{i=1}^{n} \frac{1}{\sigma_{i}^{2}}}\right\}
$$

where $R_{i}$ and $\sigma_{i}$ are the double ratio and its statistical uncertainty for each magnet cycle and $R_{\text {average }}$ is the weighted average of the double ratios calculated for four (positive chicane) or three (negative chicane) magnet cycles. Here $\sigma_{\text {total }}$ includes both systematic and statistical uncertainties to the chicane asymmetry. The systematic uncertainty is then calculated as

$$
\sigma_{\mathrm{syst}}=\sqrt{\sigma_{\text {total }}^{2}-\sigma_{\mathrm{stat}}^{2}}
$$



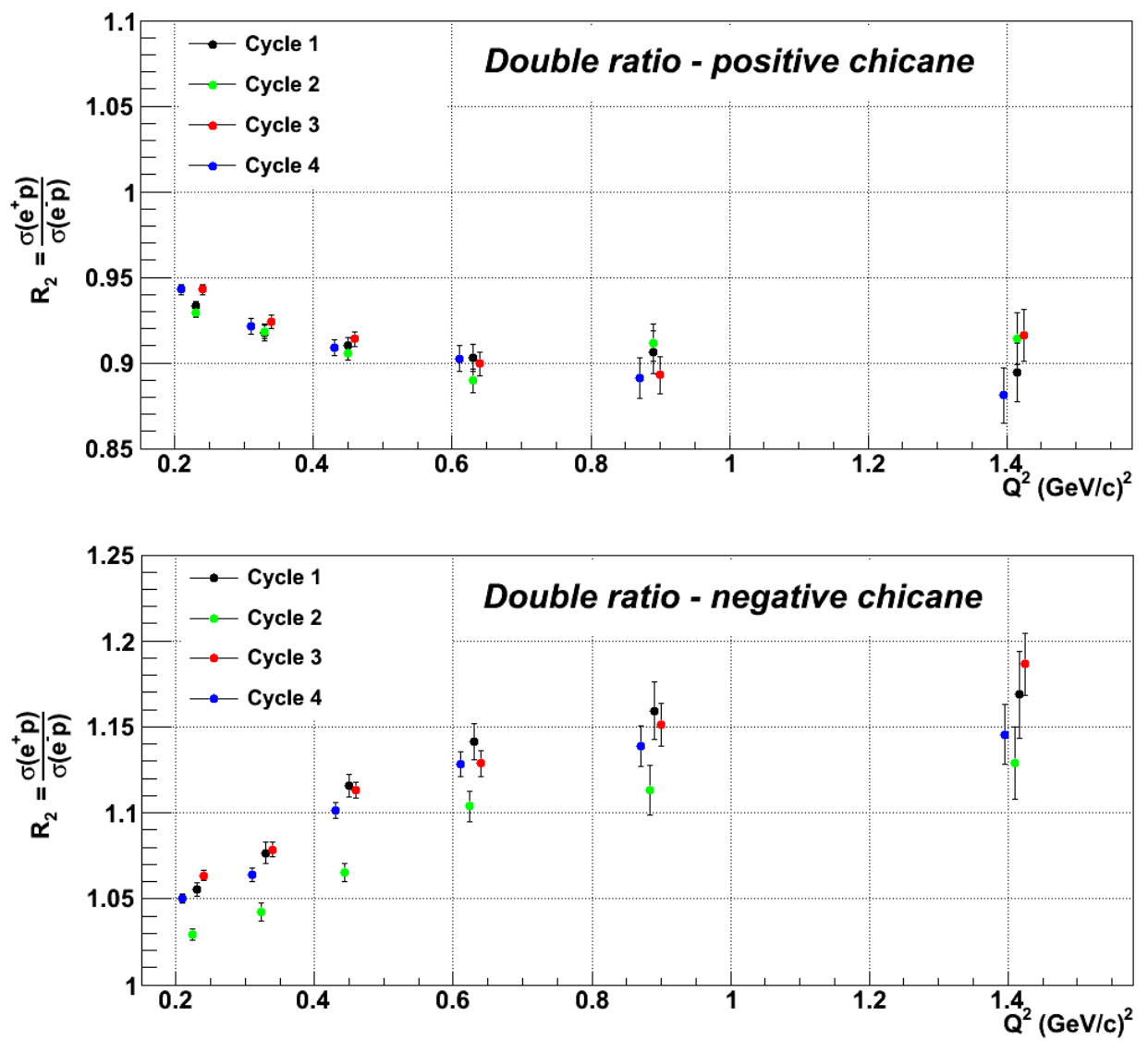

FIG. 76. The double ratios for different magnet cycles at $\varepsilon=0.88$ for (top) positive and (bottom) negative chicane magnet settings. The consistency among the double ratios for the different magnet cycles shows the consistency of the chicane. The cycle 2 negative chicane double ratios are anomalously low, indicating problems with that data set.

If the variance of the individual measurements as calculated using Eq. 65 is less than the expected variance due to the statistical uncertainties, then $\sigma_{s y s t}=0$. Note that this technique will overestimate the systematic uncertainty. In the unrealistic case where the systematic uncertainty is zero, this technique will give a non-zero $\sigma_{\text {syst }}$ half of the time.

The systematic uncertainties obtained for positive chicane $\left(\sigma_{\text {syst }}\left(R_{2}^{+}\right)\right)$and negative chicane $\left(\sigma_{\text {syst }}\left(R_{2}^{-}\right)\right)$magnet settings were then combined to calculate the 

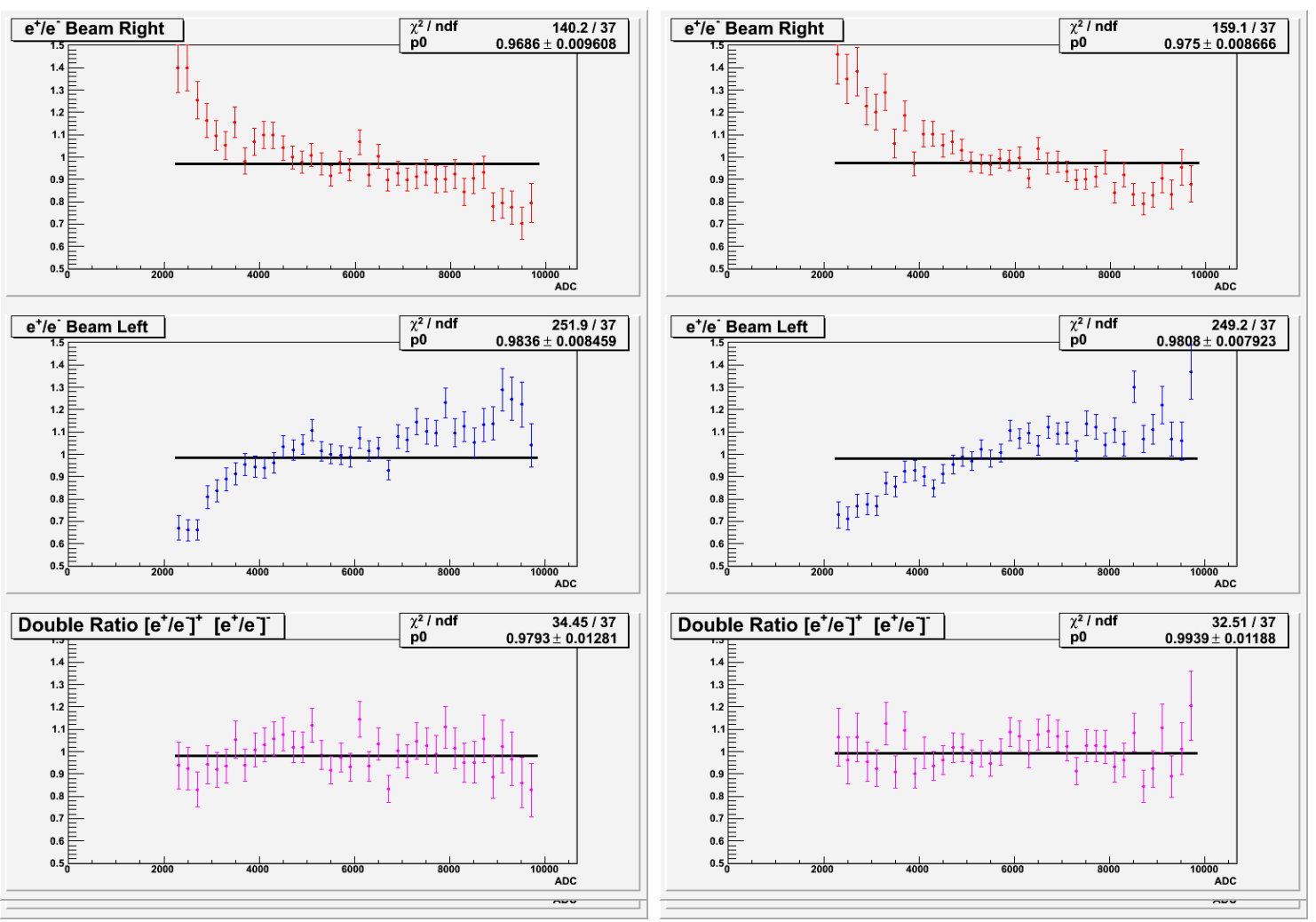

FIG. 77. The ratio of the incident positron energy density to the incident electron energy density versus incident energy (measured in channels where channel 1000 corresponds approximately to $370 \mathrm{MeV}$ ) for (top of each panel) leptons passing on the right side of the chicane before and after the Jan 14 chicane flip, (middle of each panel) the same ratio for leptons passing on the left side of the chicane, and (bottom of each panel) the product of the two ratios. The distributions are normalized to unity. The left figure is for the calorimeter at its nominal position (beam centered) and the right figure is for the calorimeter offset by approximately $2 \mathrm{~cm}$ or 0.5 modules. The statistics boxes show the results of one-parameter (constant) fits to the ratios.

total systematic uncertainties due to the asymmetry in beam transport.

$$
\sigma_{\text {syst }}(\text { chicane })=\frac{R}{\sqrt{2}} \sqrt{\left\{\left(\frac{\sigma_{\text {syst }}\left(R_{2}^{+}\right)}{R_{2}^{+}}\right)^{2}+\left(\frac{\sigma_{\text {syst }}\left(R_{2}^{-}\right)}{R_{2}^{-}}\right)^{2}\right\}}
$$

where $R_{2}^{+}$and $R_{2}^{-}$are the average double ratios for positive and negative chicane settings and the $R$ is the average quadruple ratio. The procedure was applied to 


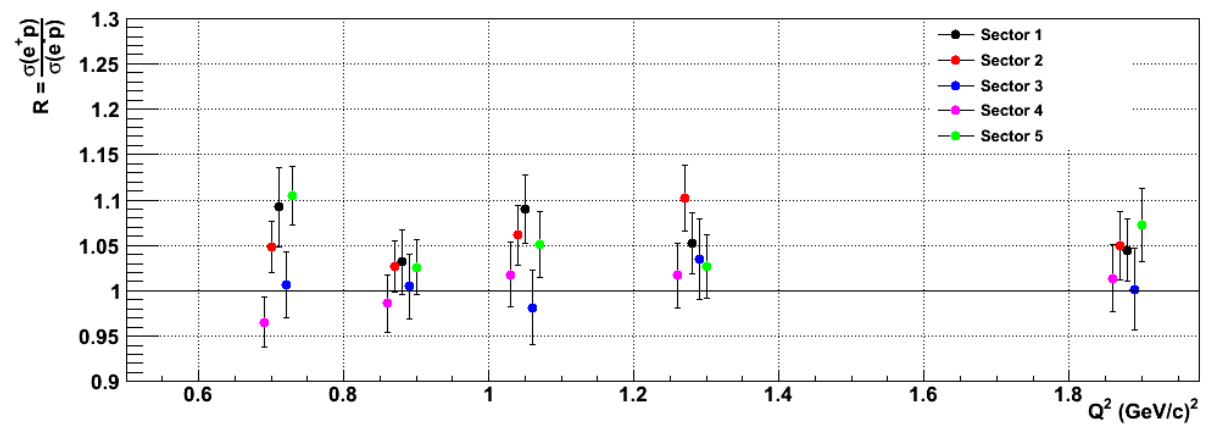

FIG. 78. The quadruple ratios for different sectors at $Q^{2}=1.45 \mathrm{GeV}^{2}$.

each kinematic bin to calculate the uncertainties due to the chicane asymmetry, $\sigma_{\text {syst }}($ chicane). See Tables 6 and 7 for the resulting uncertainties for the bins at $Q^{2}=1.45 \mathrm{GeV}^{2}$ and at $\varepsilon=0.45$. Zero uncertainties means that the variance in ratio measurements for the different chicane cycles is less than that expected due to the statistical uncertainties.

\section{CLAS detector imperfections}

There are six independent sectors in CLAS detector and therefore six independent cross section measurements. The effects of bad TOF paddles was accounted for using swimming. However, there are also other bad detector channels. In order to take these into account, we compared the quadruple ratios for the different sectors. Variations in these ratios are due to detector imperfections. We determined the systematic uncertainty due to the CLAS detector imperfections, $\sigma_{\text {syst }}$ (sector) using the variance of the sector ratios following the method of Eq. (65) and (66). See Figs. 78 and 79 and Tables 6 and 7 .

3. Background fitting region

We determined the systematic uncertainty due to background subtraction by varying the background fitting regions (this paragraph) and by varying the amount of background (next paragraph).

In the TPE data analysis these backgrounds are determined by a Gaussian function fitted to the tails of the $\Delta \phi$ distribution of the data that pass the 


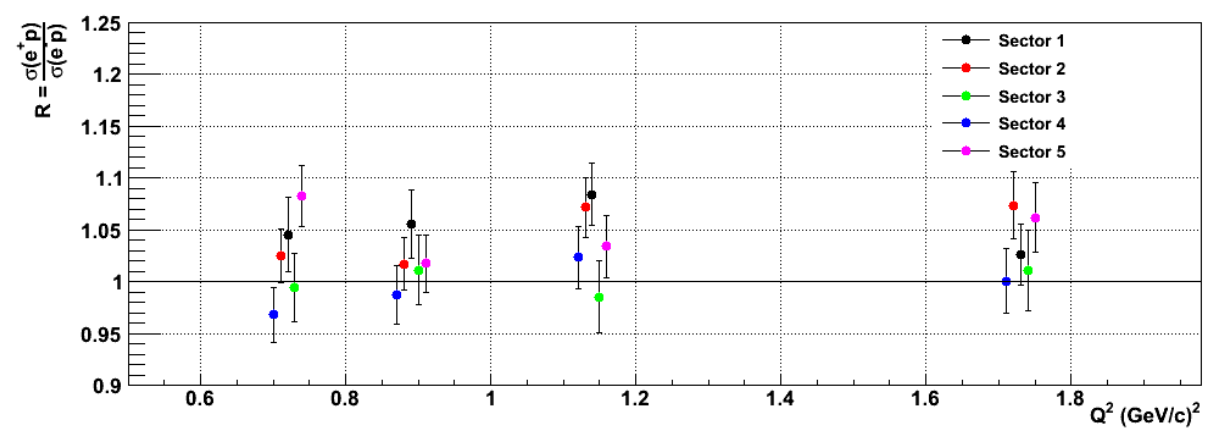

FIG. 79. The quadruple ratios for different sectors at $\varepsilon=0.45$.

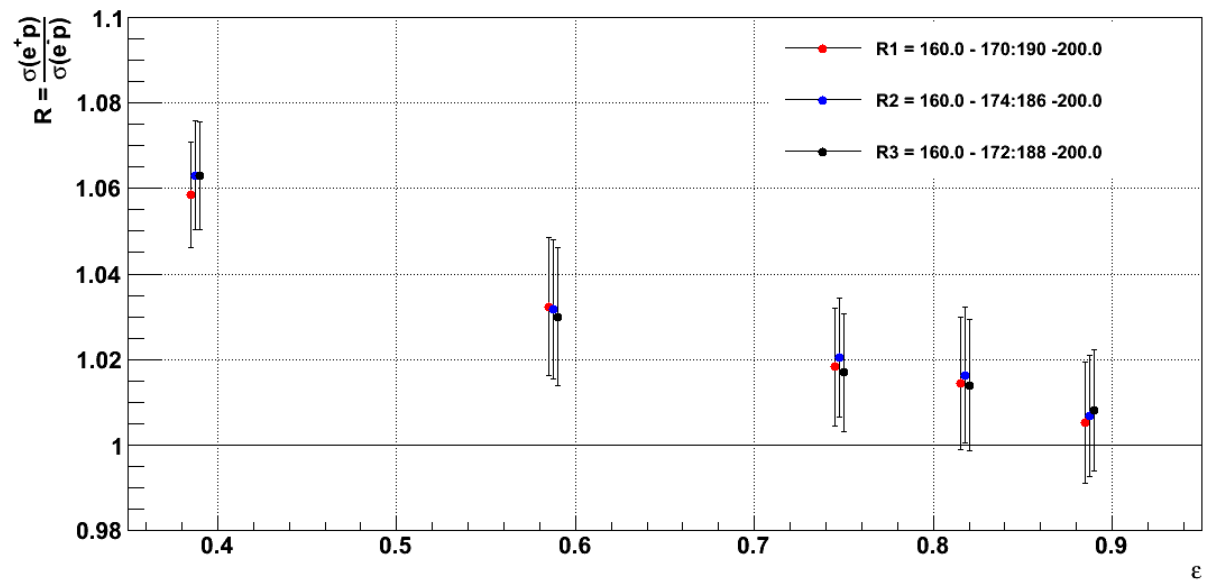

FIG. 80. The quadruple ratios at $Q^{2}=1.45 \mathrm{GeV}^{2}$ background subtracted with different fitting ranges.

other three kinematic cuts, $\Delta P_{p}$ and $\Delta E^{ \pm}$. The nominal fitting range for the tails is $160-172^{\circ}$ (left of the peak) and $188-200^{\circ}$ (right of the peak). We varied the fitting range by $-2^{\circ}\left(R_{1}=160-170: 190-200\right)$ and $+2^{\circ}$ $\left(R_{2}=160-174: 186-200\right)$ and calculated quadruple ratios (Figs. 80 and 81). The differences between the quadruple ratios obtained with those fitting ranges with the nominal $\left(\Delta R_{\text {Nominal }+2^{\circ}}, \Delta R_{\text {Nominal-2 }}\right.$ ) was averaged and assigned as systematic uncertainty due to background subtraction. See Tables 6 and 7 . 


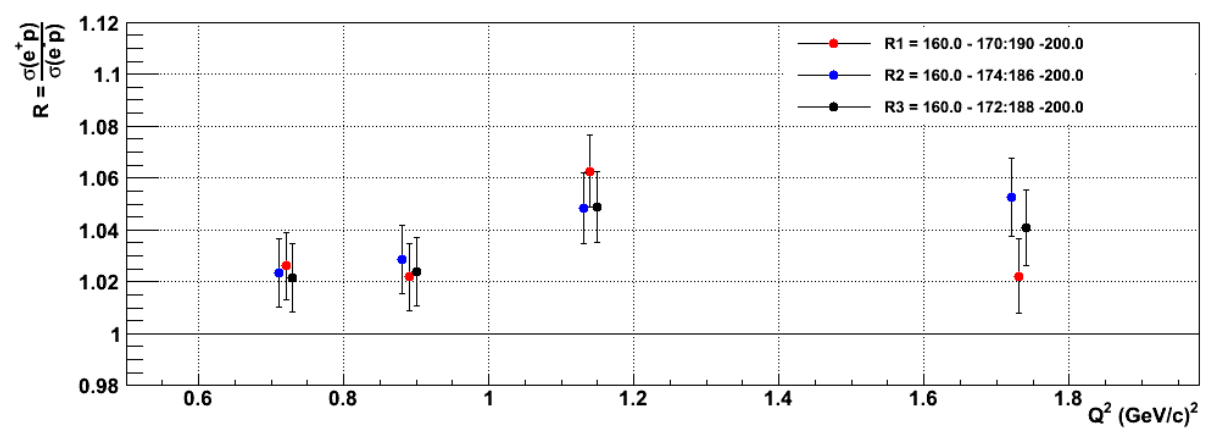

FIG. 81. The quadruple ratios at $\varepsilon=0.45$ background subtracted with different fitting ranges.

4. Elastic event selection

The final results of the ratios are calculated using the events selected with $\pm 3 \sigma$ kinematic cuts. By increasing the widths of the kinematic cuts to $\pm 3.5 \sigma$ and $\pm 4 \sigma$ we can estimate the systematic uncertainty due to the elastic event selection. Figures 82 and 84 show the quadruple ratios calculated with $3 \sigma$ and $3.5 \sigma$ kinematic cuts at $Q^{2}=1.45 \mathrm{GeV}^{2}$ and $\varepsilon=0.45$, respectively. (The backgrounds were too large at $Q^{2}=1.45 \mathrm{GeV}^{2}$ to use the $\pm 4 \sigma$ elastic cuts.) The ratios are similar in all four configurations and the amount of background approximately doubles when the widths of the kinematic cuts are increased from $3 \sigma$ to $3.5 \sigma$ (see Fig. 83). Therefore the uncertainty due to the background subtraction is also included in these uncertainties. Doubling the background at the lowest $\epsilon$ point at $Q^{2}=1.45 \mathrm{GeV}^{2}$ changed the quadruple ratio by less than $1 \%$.

The systematic uncertainty due to the elastic kinematics cuts was assigned to be the difference between the quadruple ratio determined with $3 \sigma$ cuts and that determined with $3.5 \sigma$ cuts.

5. Vertex cuts

The $30 \mathrm{~cm}$ TPE target is located $30 \mathrm{~cm}$ upstream of the CLAS center. In order to remove any contamination from non-target material such as target entrance and exit windows, we applied $-44 \mathrm{~cm}<v_{z}<-16 \mathrm{~cm}$ cuts on both lepton and proton. The vertex cuts on lepton and proton z-vertex were then tightened by 


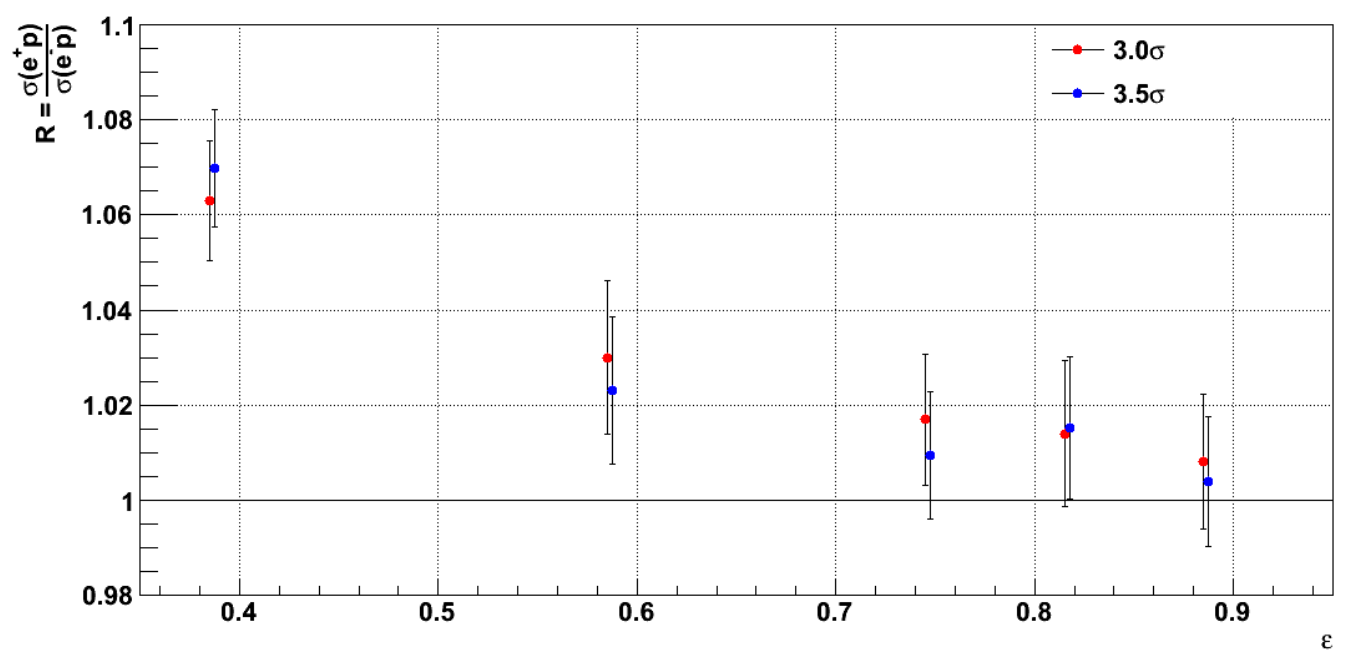

FIG. 82. The quadruple ratios at $Q^{2}=1.45 \mathrm{GeV}^{2}$ with $3 \sigma$ and $3.5 \sigma$ kinematic cuts. Note the consistency in the quadruple ratio despite the dramatic change in the background shown in Fig. 83.

$1 \mathrm{~cm}$ from both sides $\left(-43 \mathrm{~cm}<v_{z}<-17 \mathrm{~cm}\right)$ to determine the systematic uncertainty due to the vertex cuts. The difference between the quadruple ratios obtained with nominal and tighter vertex cuts was assigned as the systematic uncertainty.

6. Fiducial cuts

In order to determine its contribution to the systematics, the fiducial region was tightened by one degree with respect to the nominal fiducial cuts. The difference between the quadruple ratios obtained with nominal and tighter fiducial cuts was assigned as a systematic uncertainty. Figures 85 and 86 show the quadruple ratios with nominal and tighter fiducial cuts at $Q^{2}=1.45 \mathrm{GeV}^{2}$ and $\varepsilon=0.45$, respectively. The systematic uncertainty due to fiducial cuts are negligible compared to the other contributions at $\varepsilon<0.8$.

The calculated systematic uncertainties due to each of the above sources for the high $Q^{2}$ bins and the low $\varepsilon$ bins are listed in Tables 6 and 7 respectively. The total systematic uncertainty $\sigma_{\text {syst }}($ Total) was obtained by adding the individual contributions in quadrature. 


\begin{tabular}{|c|c|c|c|c|c|c|c|c|}
\hline$Q^{2}$ & $\varepsilon$ & $\begin{array}{c}\sigma_{\text {syst }} \\
\text { chicane }\end{array}$ & $\begin{array}{c}\sigma_{\text {syst }} \\
\text { sector }\end{array}$ & $\begin{array}{c}\sigma_{\text {syst }} \\
\text { elastic } \\
\text { event }\end{array}$ & $\begin{array}{c}\sigma_{\text {syst }} \\
\text { background }\end{array}$ & $\begin{array}{c}\sigma_{\text {syst }} \\
\text { vertex } \\
\text { cuts }\end{array}$ & $\begin{array}{c}\sigma_{\text {syst }} \\
\text { fiducial } \\
\text { cuts }\end{array}$ & $\begin{array}{l}\sigma_{\text {syst }} \\
\text { Total }\end{array}$ \\
\hline 1.44 & 0.40 & 0.0000 & 0.0095 & 0.0070 & 0.0023 & 0.0031 & 0.0003 & 0.0124 \\
\hline 1.45 & 0.60 & 0.0000 & 0.0000 & 0.0069 & 0.0021 & 0.0004 & 0.0005 & 0.0072 \\
\hline 1.46 & 0.76 & 0.0026 & 0.0043 & 0.0075 & 0.0024 & 0.0021 & 0.0005 & 0.0096 \\
\hline 1.47 & 0.83 & 0.0000 & 0.0000 & 0.0012 & 0.0014 & 0.0015 & 0.0046 & 0.0051 \\
\hline 1.47 & 0.90 & 0.0133 & 0.0000 & 0.0043 & 0.0021 & 0.0024 & 0.0057 & 0.0154 \\
\hline
\end{tabular}

TABLE 6. Systematic uncertainties on the quadruple ratio due to various sources at $Q^{2}=1.45 \mathrm{GeV}^{2}$. Zeroes indicate that the variance expected due to the statistical uncertainty was greater than the variance of the chicane or sector $e^{+} p / e^{-} p$ ratios.

\begin{tabular}{ccccccccc}
\hline \hline$Q^{2}$ & $\varepsilon$ & $\begin{array}{c}\sigma_{\text {syst }} \\
\text { chicane }\end{array}$ & $\begin{array}{c}\sigma_{\text {syst }} \\
\text { sector }\end{array}$ & $\begin{array}{c}\sigma_{\text {syst }} \\
\text { elastic } \\
\text { event }\end{array}$ & $\begin{array}{c}\sigma_{\text {syst }} \\
\text { background }\end{array}$ & $\begin{array}{c}\sigma_{\text {syst }} \\
\text { vertex } \\
\text { cuts }\end{array}$ & $\begin{array}{c}\sigma_{\text {syst }} \\
\text { fiducial } \\
\text { cuts }\end{array}$ & $\begin{array}{c}\sigma_{\text {syst }} \\
\text { Total }\end{array}$ \\
\hline 0.72 & 0.45 & 0.0086 & 0.0156 & 0.0033 & 0.0033 & 0.0003 & 0.0001 & 0.0185 \\
0.89 & 0.45 & 0.0000 & 0.0000 & 0.0132 & 0.0034 & 0.0057 & 0.0001 & 0.0148 \\
1.14 & 0.45 & 0.0000 & 0.0000 & 0.0037 & 0.0071 & 0.0015 & 0.0004 & 0.0082 \\
1.73 & 0.45 & 0.0135 & 0.0000 & 0.0063 & 0.0115 & 0.0012 & 0.0007 & 0.0189 \\
\hline \hline
\end{tabular}

TABLE 7. Systematic uncertainties on the quadruple ratio due to various sources at $\varepsilon=0.45$. Zeroes indicate that the variance expected due to the statistical uncertainty was greater than the variance of the chicane or sector $e^{+} p / e^{-} p$ ratios. 

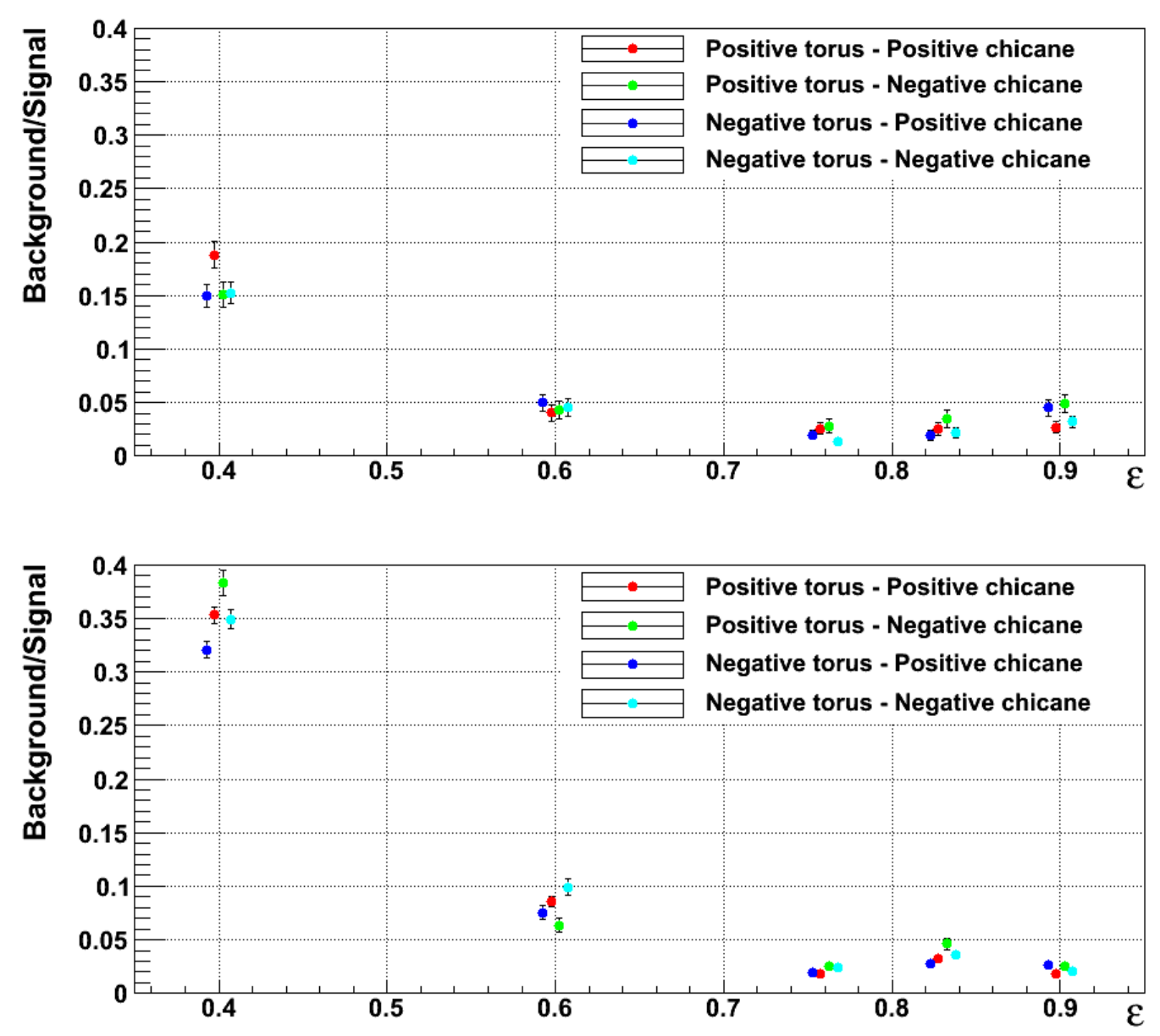

FIG. 83. Background to signal ratios at $Q^{2}=1.45 \mathrm{GeV}^{2}$ with $3 \sigma$ (top) and $3.5 \sigma$ (bottom) kinematic cuts. The proportion of background approximately doubles as the cuts are loosened slightly.

\subsection{RADIATIVE CORRECTIONS}

The higher order radiative corrections shown in Fig. 8 have a significant impact on the elastic lepton-proton cross sections. The largest contributions among those radiative corrections are the charge even terms, which are independent of the charges of the lepton and proton. Since we are comparing the positron-proton to electronproton cross sections, these charge-even terms cancel in the ratio. The charge-odd terms do not cancel and must be calculated. 


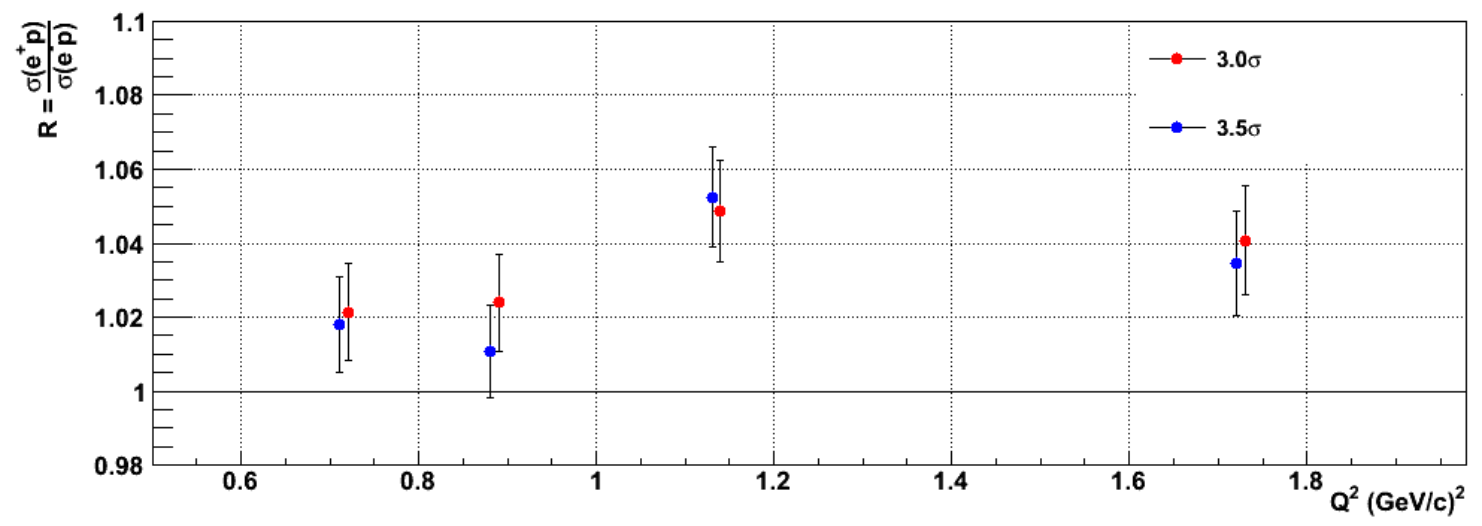

FIG. 84. The quadruple ratios at $\varepsilon=0.45$ with $3 \sigma$ and $3.5 \sigma$ kinematic cuts.

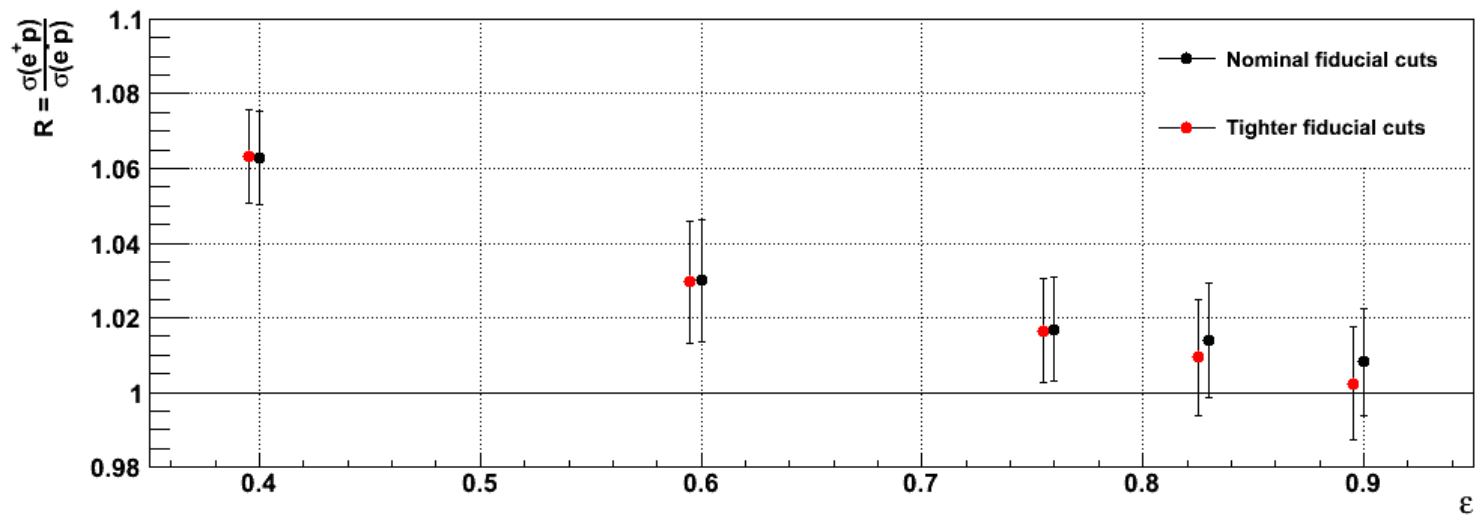

FIG. 85. The quadruple ratios at $Q^{2}=1.45 \mathrm{GeV}^{2}$ with nominal and tighter fiducial cuts.

There are two leading order charge-odd correction terms to the Born approximation (see Fig. 8). The first one is the interference between the Born and TPE amplitudes $\left(\delta_{2 \gamma}\right)$ and the second one is the interference between the lepton-proton bremstrahlung amplitudes $\left(\delta_{\text {e.p.br }}\right)$. We want to measure $\delta_{2 \gamma}$, therefore we need to compute $\delta_{\text {e.p.br}}$. Since the proton bremstrahlung has some sensitivity to proton internal structure, $\delta_{\text {e.p.br }}$ is also slightly model dependent. At low $Q^{2}$, this sensitivity is relatively small.

Any of the three particles, the incident and scattered electrons and the proton, can 


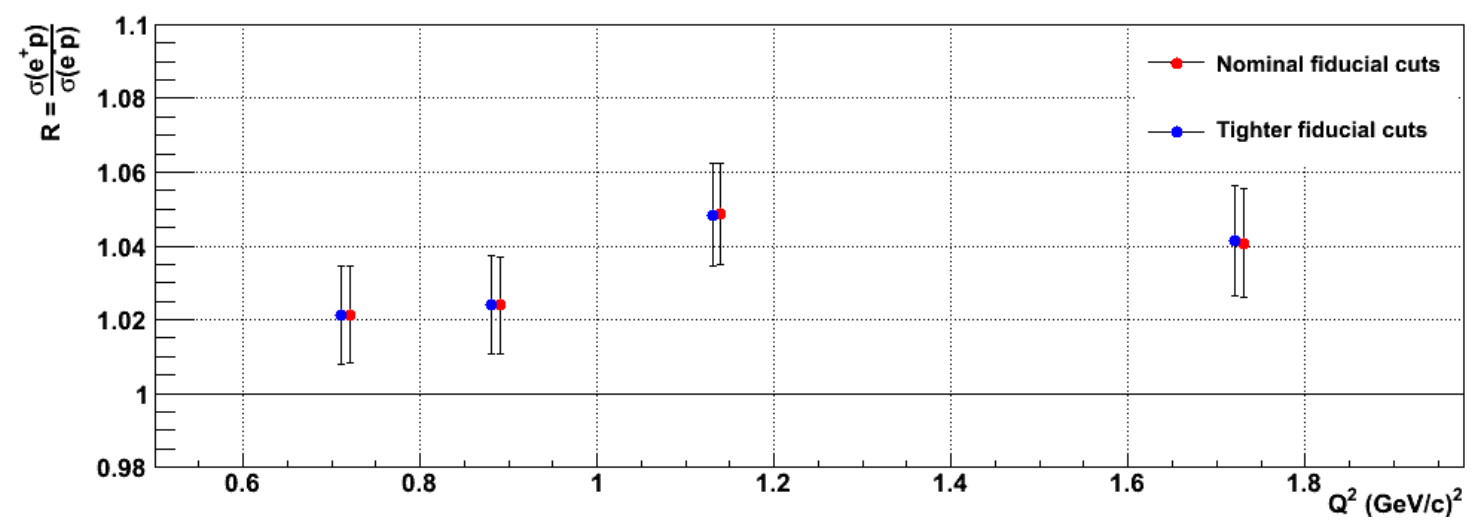

FIG. 86. The quadruple ratios at $\varepsilon=0.45$ with nominal and tighter fiducial cuts.

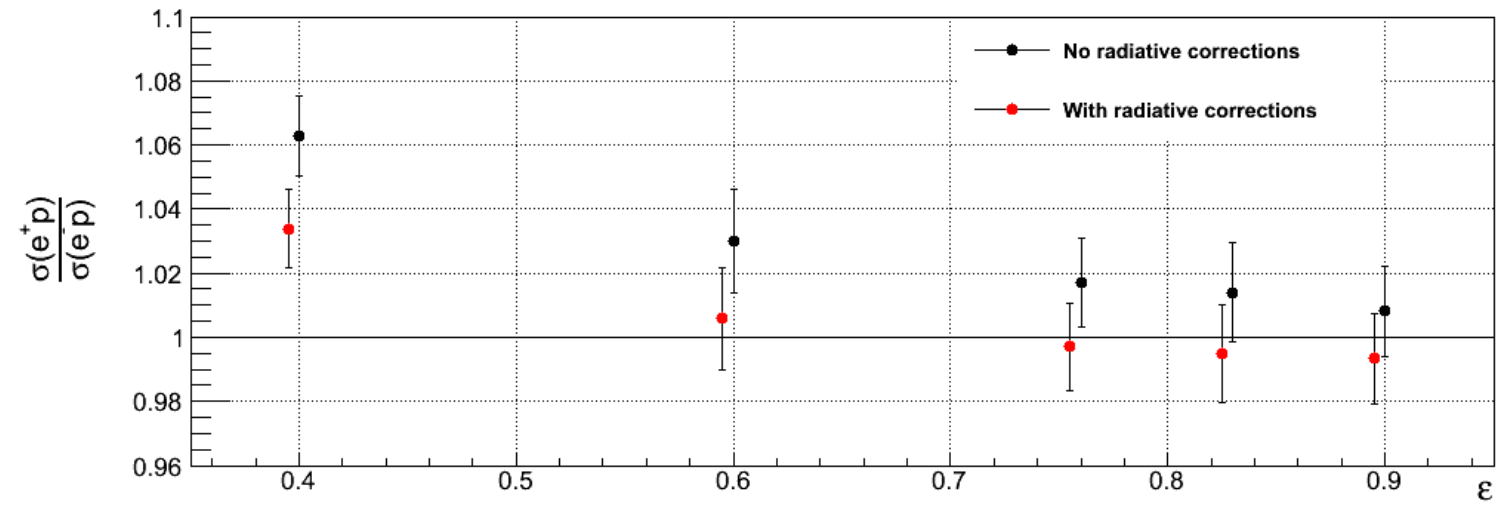

FIG. 87. The quadruple ratios at $Q^{2}=1.45 \mathrm{GeV}^{2}$ with and without radiative corrections.

radiate a bremstrahlung photon. In order to calculate these bremstrahlung contributions, we simulate radiative effects following the prescription of [41]. This approach is called the 'extended peaking approximation approach' and it allows generation of radiated photons only in the directions of the charged particles. Because the incoming and outgoing leptons and the struck proton are all allowed to radiate, the sum of the radiated photon momenta has a fairly realistic angular distribution. Typically, these simulations use fixed energy loss or invariant mass $\left(W^{2}\right)$ cut to determine which events are included as elastic and which are in the excluded radiative tail. In our 


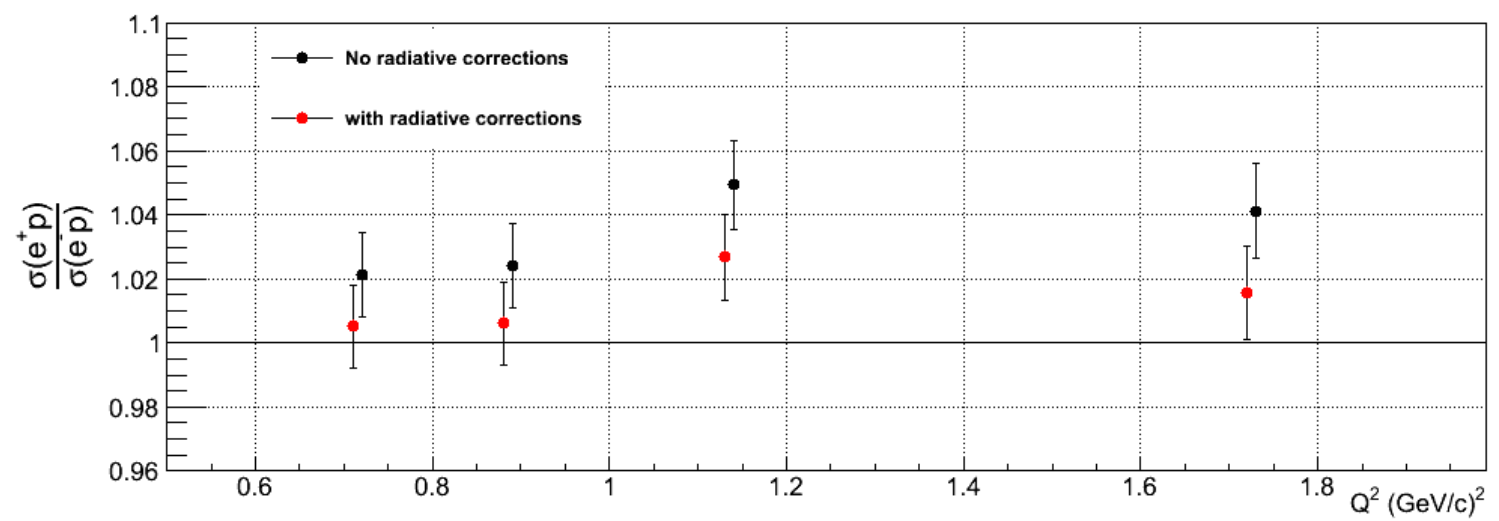

FIG. 88. The quadruple ratios at $\varepsilon=0.45$ with and without radiative corrections.

case, we used the same four kinematic cuts $\left(\Delta \phi, \Delta P_{p}, \Delta E+E_{e}\right.$ and $\left.\Delta E-E_{e}\right)$ which we applied to the TPE data to identify elastic events.

The measured cross section ratio, $\sigma\left(e^{+} p\right) / \sigma\left(e^{-} p\right)$, was divided by the calculated ratio of the radiated $e^{+} p$ to the radiated $e^{-} p$ cross section. The radiative corrections at $Q^{2}=1.45 \mathrm{GeV} / \mathrm{c}^{2}$ and $\varepsilon=0.4$ is consistent with the radiative corrections applied to the Novosibirsk results at similar kinematics. The effects of radiative corrections on the data can be seen in Figs. 87 and 88 . The uncertainties of the radiative corrections were estimated by varying the widths of the four TPE kinematic cuts from $3 \sigma$ to $3.5 \sigma$. The difference between the radiatively corrected cross section ratio $(R)$ with $3 \sigma$ and $3.5 \sigma,\left|R_{3 \sigma}-R_{3.5 \sigma}\right|$, was taken as the uncertainty and was $\lesssim 0.2 \%$ for all bins. 


\section{CHAPTER 6}

\section{DISCUSSION AND CONCLUSION}

\subsection{DISCUSSION}

The CLAS TPE experiment measured the positron-proton to electron-proton elastic cross section ratios in order to determine the TPE contribution to the elasticlepton scattering cross section. This thesis described the experimental technique and data analysis process in detail. The positron-proton to electron-proton cross section ratios $(R)$ at $Q^{2}>1 \mathrm{GeV} / \mathrm{c}^{2}$ and at $\varepsilon<0.65$ were presented, along with the estimations of systematic uncertainties and radiative corrections. Tables 8 and 9 show the final results obtained for $R$ and the associated statistical, systematic and total uncertainties for each bin. The total uncertainties are $\lesssim 2 \%$ for all bins.

\begin{tabular}{ccccccc}
\hline \hline $\operatorname{Bin}$ & $Q^{2}$ & $\varepsilon$ & $\mathrm{R}$ & $\sigma_{\text {stat }}$ & $\sigma_{\text {syst }}$ & $\sigma_{\text {Total }}$ \\
\hline 1 & 1.44 & 0.40 & 1.0339 & 0.0126 & 0.0124 & 0.0177 \\
2 & 1.45 & 0.60 & 1.0058 & 0.0162 & 0.0072 & 0.0177 \\
3 & 1.46 & 0.76 & 0.9979 & 0.0138 & 0.0096 & 0.0168 \\
4 & 1.47 & 0.83 & 0.9951 & 0.0154 & 0.0051 & 0.0162 \\
5 & 1.47 & 0.90 & 0.9933 & 0.0142 & 0.0154 & 0.0209 \\
\hline \hline
\end{tabular}

TABLE 8. Final $e^{+} p / e^{-} p$ cross section ratios $(\mathrm{R})$ and the associated statistical $\left(\sigma_{\text {stat }}\right)$, systematic $\left(\sigma_{\text {syst }}\right)$ and total uncertainties $\left(\sigma_{\text {Total }}\right)$ at $Q^{2}=1.45 \mathrm{GeV}^{2}$. Charge-odd radiative corrections have been applied.

\subsubsection{COMPARISON WITH PREVIOUS MEASUREMENTS AND THEORETICAL CALCULATIONS}

We also compared our results with previous measurements and with theoretical calculations. Fig. 89 compares our $Q^{2}=1.45 \mathrm{GeV}^{2}$ measurements with the previous 


\begin{tabular}{ccccccc}
\hline \hline $\operatorname{Bin}$ & $Q^{2}$ & $\varepsilon$ & $\mathrm{R}$ & $\sigma_{\text {stat }}$ & $\sigma_{\text {syst }}$ & $\sigma_{\text {Total }}$ \\
\hline 1 & 0.72 & 0.45 & 1.0049 & 0.0131 & 0.0185 & 0.0227 \\
2 & 0.89 & 0.45 & 1.0060 & 0.0130 & 0.0148 & 0.0197 \\
3 & 1.14 & 0.45 & 1.0260 & 0.0137 & 0.0082 & 0.0159 \\
4 & 1.73 & 0.45 & 1.0150 & 0.0146 & 0.0189 & 0.0238 \\
\hline \hline
\end{tabular}

TABLE 9. Final $e^{+} p / e^{-} p$ cross section ratios $(\mathrm{R})$ and the associated statistical $\left(\sigma_{\text {stat }}\right)$, systematic $\left(\sigma_{\text {syst }}\right)$ and total uncertainties $\left(\sigma_{\text {Total }}\right)$ at $\varepsilon=0.45$. Charge-odd radiative corrections have been applied.

world measurements taken at $Q^{2}=1-2 \mathrm{GeV}^{2}$. There are only few previous measurements available for that kinematic region. The Novosibirsk measurement is the most precise measurement among those. Even though our data points have slightly higher uncertainties than the Novosibirsk measurement, CLAS TPE covered a wide range of $\varepsilon$ and is more precise than other previous world measurements.

The theoretical calculations shown in Fig 89 were done by Blunden, Melnitchouk and Tjon [11] assuming the nucleon intermediate state in their hadronic framework. The CLAS TPE measurements are consistent with those theoretical calculations. We also compared our results at $\varepsilon=0.45$ with previous measurements (see Fig. 90) and theoretical calculation [11]. There were only three measurements available for that kinematic region and our results are more precise than those previous measurements. The CLAS TPE results at $\varepsilon=0.45$ also are in good agreement with theoretical predictions by Blunden, Melnitchouk and Tjon.

The high $\varepsilon$ and the $Q^{2} \approx 0.85 \mathrm{GeV}^{2}$ data of the CLAS TPE experiment were analyzed by Dipak Rimal at Florida International University [39]. He observed a slight $Q^{2}$ dependency of the positron-proton to electron-proton cross section ratios at $\varepsilon=0.88$ [39] and those $R$ measurement were also more precise than the previous world measurements and agreed with the theoretical calculation by Blunden et al. [11]. Rimal found no significant $\varepsilon$ dependance of $R$ at $Q^{2}=0.85 \mathrm{GeV}^{2}$. 


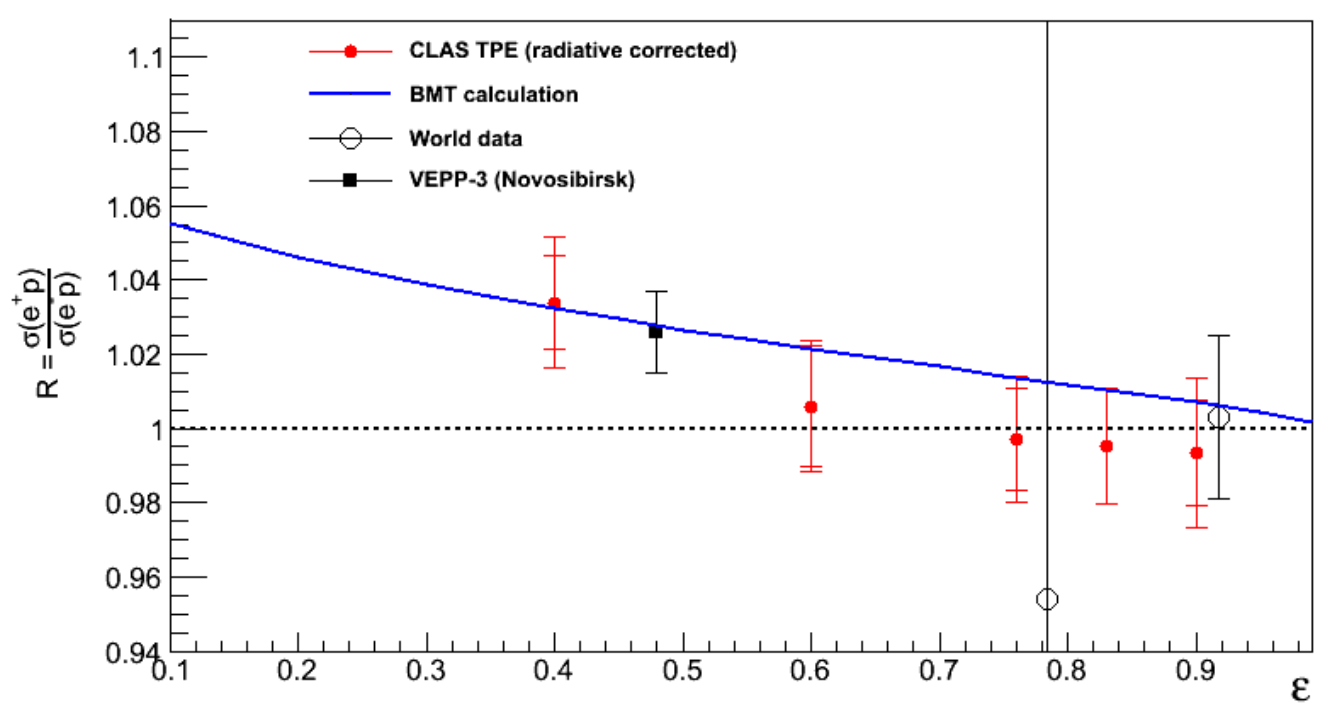

FIG. 89. The positron-proton to electron-proton cross section ratios obtained from this experiment at $Q^{2}=1.45 \mathrm{GeV}^{2}$ (red) compared with previous measurements at $Q^{2}=1-2 \mathrm{GeV}^{2}$ (black) and a theoretical calculation by Blunden et al. [11].

\subsubsection{IMPLICATIONS OF THE TPE MEASUREMENTS ON THE ELASTIC ELECTRON-PROTON CROSS SECTION}

Figure 91 shows the positron-proton to electron-proton elastic cross section ratios measured at $Q^{2}=1.45 \mathrm{GeV}^{2}$ with a linear fit. The linear fit was used to determine the elastic cross section ratio as a function of $\varepsilon$. The radiatively corrected elastic cross section ratio, $R$, determined by the linear fit was used to calculate the TPE contribution $\left(\delta_{2 \gamma}\right)$ and correct the measured elastic scattering cross sections at each value of $\varepsilon$ as below.

$$
\begin{aligned}
\sigma\left(e^{-} p\right) & =\sigma_{\text {Born }}\left(1+\delta_{2 \gamma}\right) \\
\sigma\left(e^{+} p\right) & =\sigma_{\text {Born }}\left(1-\delta_{2 \gamma}\right) \\
R & =\frac{\sigma\left(e^{+} p\right)}{\sigma\left(e^{-} p\right)}=1-2 \delta_{2 \gamma}
\end{aligned}
$$

We applied the CLAS TPE corrections to the Rosenbluth separation measurements at $Q^{2}=1.75 \mathrm{GeV}^{2}$ [4]. Figure 92 shows the reduced cross section measurements normalized by the square of the dipole form factor $\left(G_{D}^{2}\right)$ as a function of $\varepsilon$ 


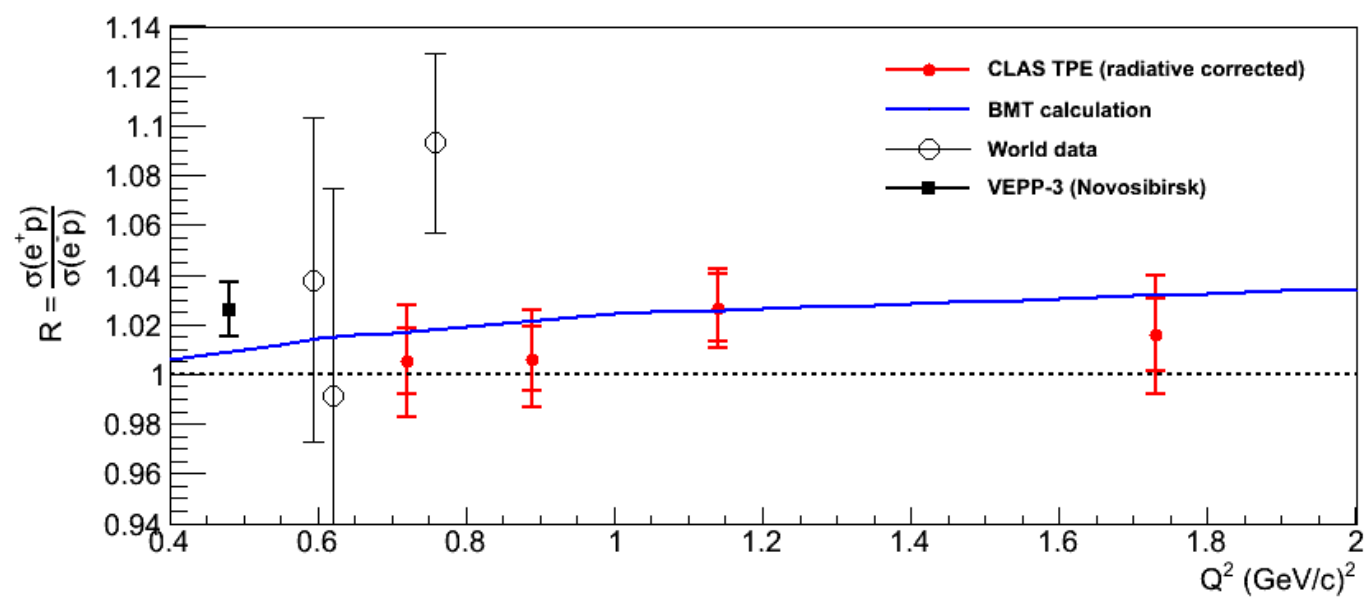

FIG. 90. The positron-proton to electron-proton cross section ratios obtained from this experiment at $\varepsilon=0.45$ (red) compared with previous measurements (black) and a theoretical calculation by Blunden et al. [11].

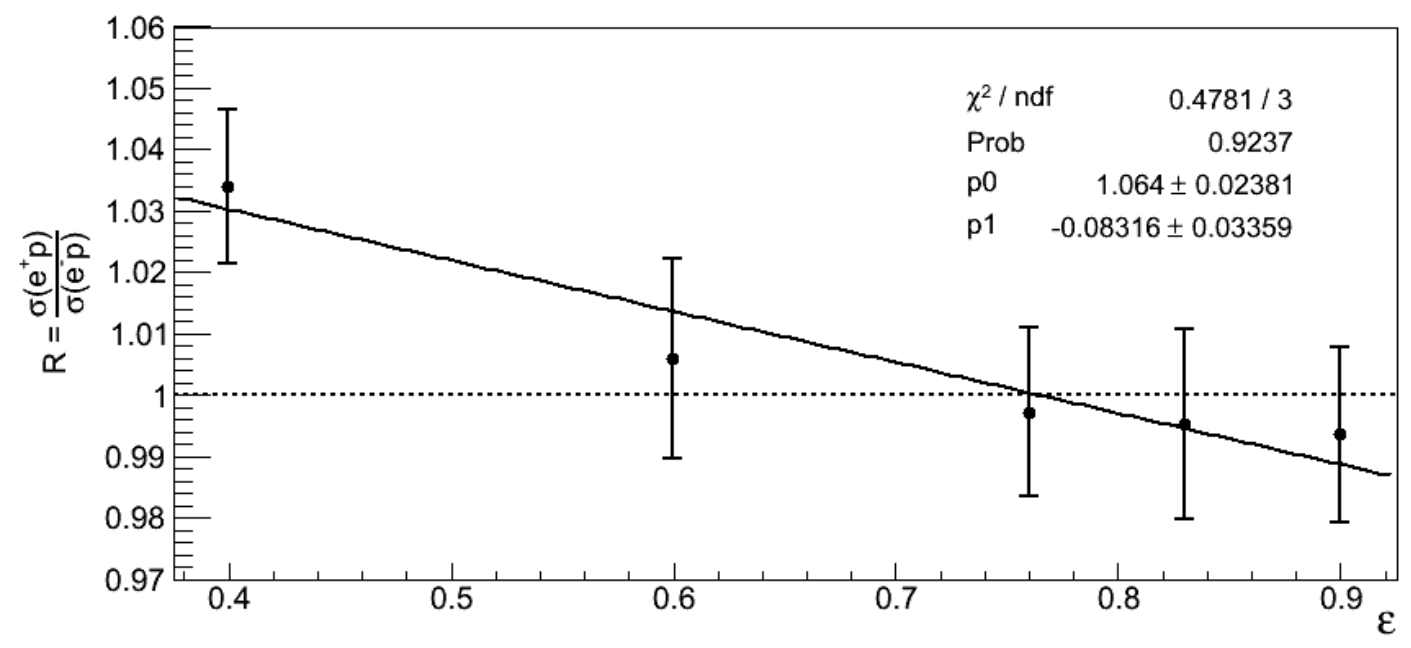

FIG. 91. The quadruple ratio as a function of $\varepsilon$ at $Q^{2} \sim 1.45 \mathrm{GeV}^{2}$ with a linear fit.

at $Q^{2}=1.75 \mathrm{GeV}^{2}$. The black data and the curve show the original measurement from Ref. [4] and the linear fit to the data. The blue data represents the TPE corrected cross section measurements. The original and the TPE corrected $\mu_{p} G_{E} / G_{M}$ 


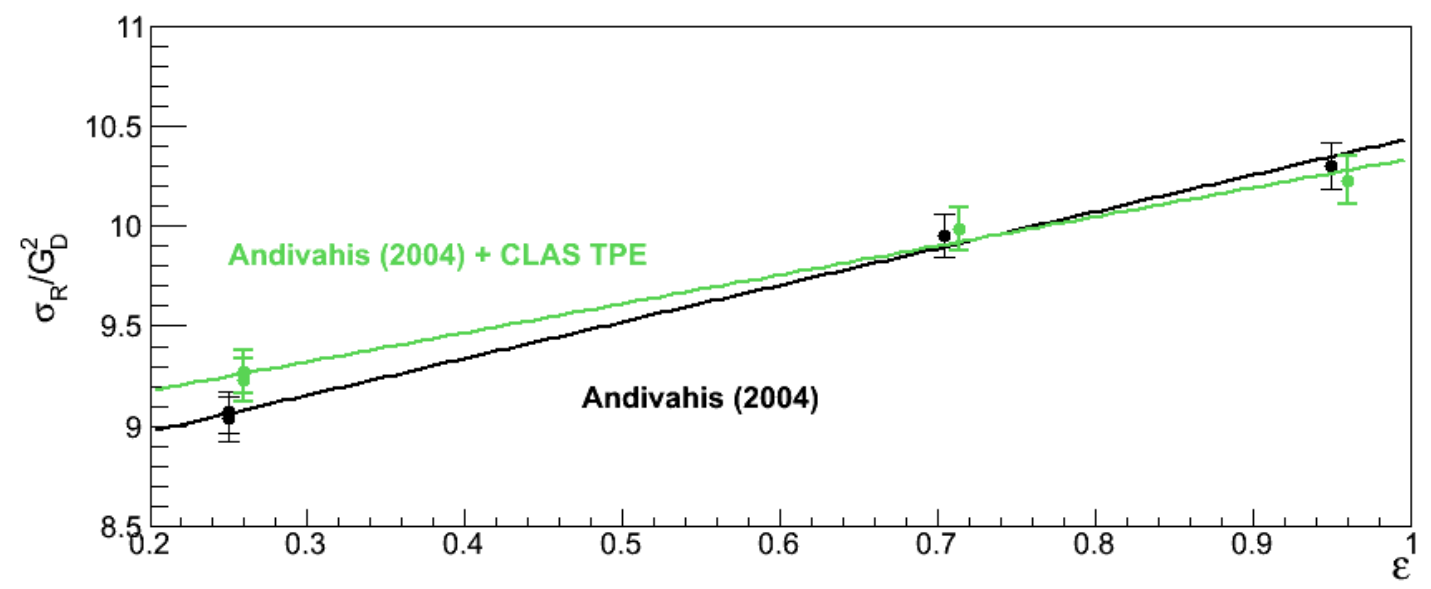

FIG. 92. The original (black) and TPE corrected (blue) reduced cross section, normalized by the square of the dipole form factor $\left(G_{D}^{2}\right)$, measured by Andivahis et al. [4] as function of $\varepsilon$. The slopes and intercepts of the linear fits are used to determined the $\mu_{p} G_{E} / G_{M}$.

ratios were calculated using the slope and the intercept of the linear fits. The slope gives $\frac{G_{E}^{2}}{\mu_{p} G_{D}^{2}}$ and the intercept gives $\frac{G_{M}^{2}}{G_{D}^{2}}$. The results are given in Table 10. The TPE corrections change the intercept by only a few percent, but the slope significantly $(\sim 20 \%)$.

We also compared the TPE corrected and original Rosenbluth separation measurements at $Q^{2}=1.75 \mathrm{GeV}^{2}$ with $\mu_{p} G_{E} / G_{M}$ ratio measured by the polarization transfer technique at $Q^{2}=1.77 \mathrm{GeV}^{2}$. As shown in Fig. 93, the CLAS TPE corrections bring the Rosenbluth separation and the polarization measurements at $Q^{2} \sim 1.75 \mathrm{GeV}^{2}$ into excellent agreement.

To estimate the uncertainties due to the inclusion of the CLAS TPE corrections, we vary the slope of the TPE correction to the elastic cross section ratios by $\pm 1 \sigma$. See Fig. 94. There is no significant uncertainty of the intercept of the linear fit and therefore that is neglected in the uncertainty calculation. TPE corrected $\frac{\mu_{p} G_{E}}{G_{M}}$ were determined from those cases. The difference between proton form factors calculated from the case 1 and the nominal, $\left|\left(\frac{\mu_{p} G_{E}}{G_{M}}\right)_{\text {case } 1}-\left(\frac{\mu_{p} G_{E}}{G_{M}}\right)_{\text {nominal }}\right|$, was taken as the upper bound of the uncertainty due to the TPE correction. Similarly, $\left|\left(\frac{\mu_{p} G_{E}}{G_{M}}\right)_{\text {case 2 }}-\left(\frac{\mu_{p} G_{E}}{G_{M}}\right)_{\text {nominal }}\right|$ was taken as the lower bound of the uncertainty due 


\begin{tabular}{lcccc}
\hline \hline & Slope & Intercept & $\frac{\mu_{p} G_{E}}{G_{M}}$ & Total error \\
\hline Andivahis et al. & 1.839 & 8.6010 & 0.910 & +0.059 \\
& & & & -0.061 \\
Andivahis et al. + CLAS TPE corrections & 1.454 & 8.8789 & 0.796 & +0.067 \\
& & & & -0.085 \\
\hline \hline
\end{tabular}

TABLE 10. $\frac{\mu_{p} G_{E}}{G_{M}}$ measurements at $Q^{2}=1.75 \mathrm{GeV}^{2}$ by Andivahis et al. [4] without and with CLAS TPE corrections.

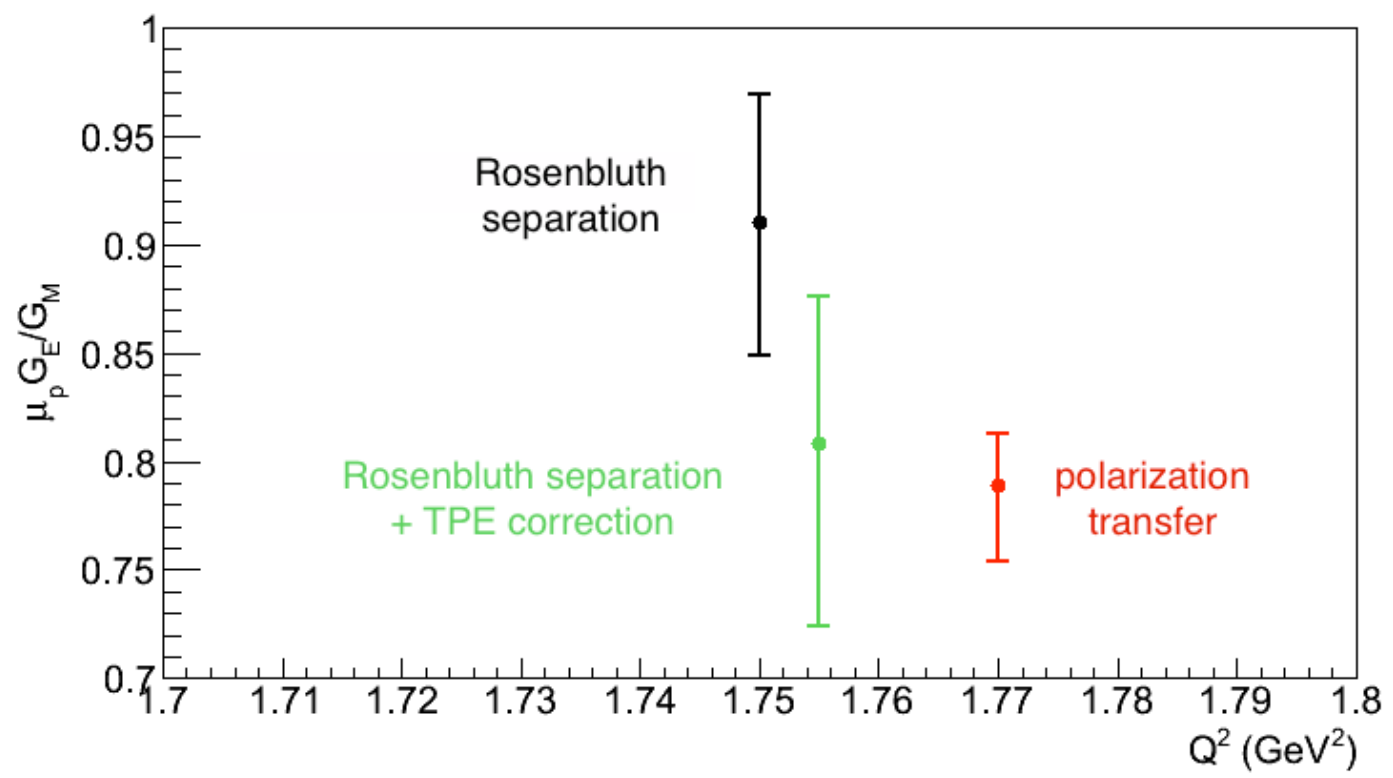

FIG. 93. The proton form factor ratio, $\frac{\mu_{p} G_{E}}{G_{M}}$, measured by Rosenbluth separation method [4] (black) with (blue) and without (black) CLAS TPE corrections and measured by polarization transfer technique [20] (red). The TPE corrected proton form factor ratio point includes the correction uncertainty. See text for details.

to the TPE correction. Uncertainties due to the TPE corrections were added to the total uncertainties of the original Rosenbluth separation measurement in quadrature ( see Table 10). 


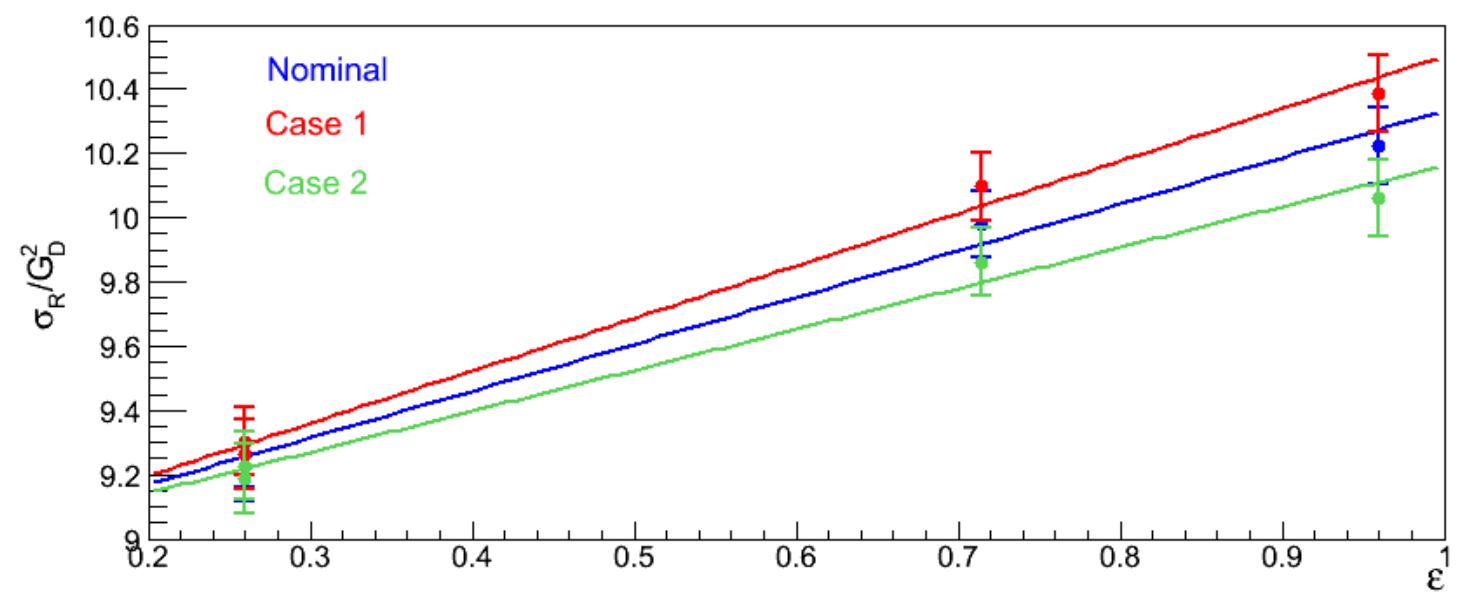

FIG. 94. TPE corrected reduced cross sections as a function of $\varepsilon$ at $Q^{2} \sim 1.75$ $\mathrm{GeV}^{2}$ with additional cases. In the nominal case, the TPE correction at a given $\varepsilon$ is determined by the linear fit to the CLAS TPE elastic cross section ratios shown in Fig. 91. In cases 1 and 2, we varied the slope of the linear fit by $\pm \sigma$.

\subsection{CONCLUSION}

The CLAS TPE experiment measured the positron-proton to electron-proton elastic cross section ratios using simultaneous electron-positron beam produced at Jefferson Lab Hall B. The elastic scattering data were analyzed with associated systematic uncertainties, background subtraction and other required corrections. In this dissertation I presented the positron-proton to electron-proton elastic scattering cross section ratios at $Q^{2}=1.45 \mathrm{GeV}^{2}$ and at $\varepsilon=0.45$ with an uncertainty of $\lesssim 2 \%$. These ratios showed a significant $\varepsilon$ dependence at $Q^{2}=1.45 \mathrm{GeV}^{2}$ and no $Q^{2}$ dependence at $\varepsilon=0.45$.

Our results at $Q^{2}=1.45 \mathrm{GeV}^{2}$ and the Novosibirsk measurement are consistent with the previous world measurements and with theoretical predictions by Blunden, Melnitchouk and Tjon. Their TPE calculations were performed in the hadronic framework with nucleon elastic intermediate states. These hadronic calculations are reliable for all $\varepsilon$ values at low $Q^{2}$. For $Q^{2} \lesssim 2-3 \mathrm{GeV} / \mathrm{c}^{2}$, the TPE corrections to the cross section bring the form factor ratio extracted from Rosenbluth separation measuremets into excellent agreement with the polarization transfer measurements [8] (see middle panel of Fig. 95). At higher $Q^{2}$, the calculation is expected to be less 


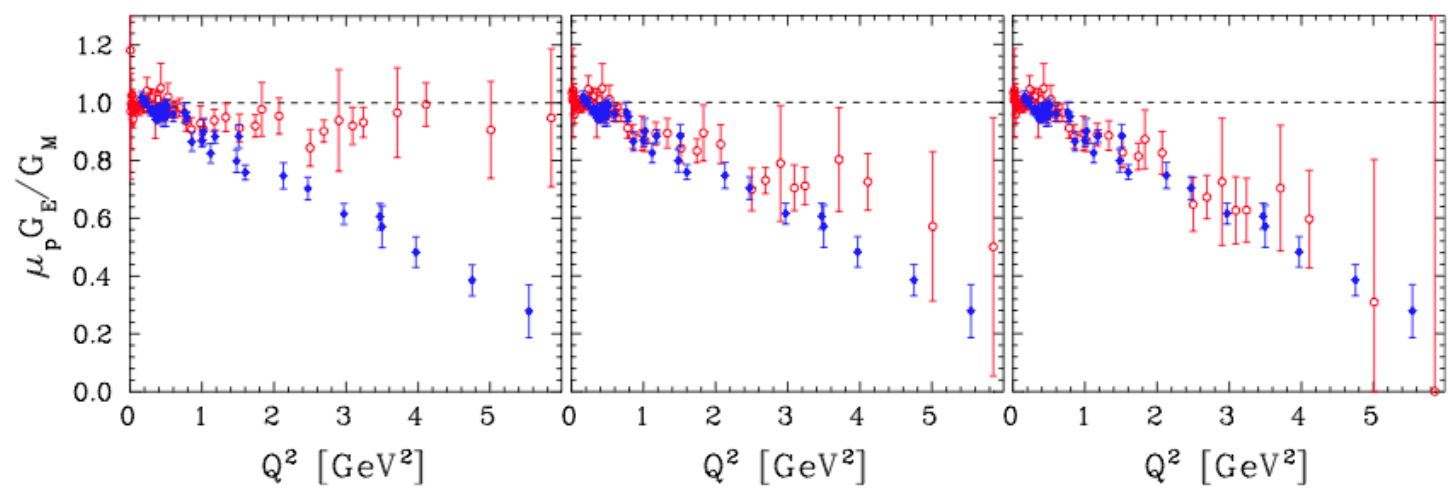

FIG. 95. Comparison of polarization measurements (filled diamonds) and Rosenbluth separation measurements (open circles) with no TPE corrections (left), TPE corrections from Blunden et al. [42] (center), and with the additional high $Q^{2}$ correction applied from Arrington et al. [43] (right)

complete and therefore a small additional phenomenological correction term associated with the higher-mass intermediate state contributions is needed to explain the $G_{E}$ discrepancy [8].

The TPE corrections determined by the CLAS TPE experiment at $Q^{2}=1.45$ $\mathrm{GeV}^{2}$ were directly applied to the Rosenbluth separation cross section measurements at $Q^{2}=1.75 \mathrm{GeV}^{2}$. This significantly decreased the extracted value of $G_{E} / G_{M}$ and brought it into excellent agreement with that determined from the polarization transfer measurement.

Therefore, it appears that the two photon exchange correction can reconcile the Rosenbluth and polarization transfer measurements of the proton form factor ratio, $\mu_{p} G_{E} / G_{M}$, at momentum transfers $Q^{2}$ up to $2 \mathrm{GeV}^{2}$. We need more data and more exact calculations at higher $Q^{2}$ to explain and resolve the entire proton form factor discrepancy. 


\section{BIBLIOGRAPHY}

[1] J. J. Kelly, Phys. Rev. C 70, 068202 (2004).

[2] M. N. Rosenbluth, Phys. Rev. 79, 615 (1950).

[3] I. A. Qattan, Precision Rosenbluth measurement of proton elastic electromagnetic form factors and their ratio, Ph.D. thesis, Northwestern University (2005).

[4] L. Andivahis et al., Phys. Rev. D 50, 5491 (1994).

[5] J. Arrington, Phys. Rev. C 69, 022201 (2004).

[6] M. K. Jones et al., Phys. Rev. Lett. 84, 1398 (2000).

[7] I. A. Qattan et al., Phys. Rev. Lett. 94, 142301 (2005).

[8] J. Arrington, P. Blunden, and W. Melnitchouk, Progress in Particle and Nuclear Physics 66, 782 (2011).

[9] L. W. Mo and Y. S. Tsai, Rev. Mod. Phys. 41, 205 (1969).

[10] L. C. Maximon and J. A. Tjon, Phys. Rev. C 62, 054320 (2000).

[11] P. G. Blunden, W. Melnitchouk, and J. A. Tjon, Phys. Rev. Lett. 91, 142304 (2003).

[12] P. A. M. Guichon and M. Vanderhaeghen, Phys. Rev. Lett. 91, 142303 (2003).

[13] J. Arrington, Phys. Rev. C 71, 015202 (2005).

[14] S. Kondratyuk, P. G. Blunden, W. Melnitchouk, and J. A. Tjon, Phys. Rev. Lett. 95, 172503 (2005).

[15] S. Kondratyuk and P. G. Blunden, Phys. Rev. C 75, 038201 (2007).

[16] Y. Chen et al., Phys. Rev. Lett. 93, 122301 (2004).

[17] A. Afanasev et al., Phys. Rev. D 72, 013008 (2005).

[18] O. Gayou et al., Phys. Rev. Lett. 88, 092301 (2002).

[19] N. Kivel and M. Vanderhaeghen, Phys. Rev. Lett. 103, 092004 (2009). 
[20] V. Punjabi et al., Phys. Rev. C 71, 055202 (2005).

[21] D. Borisyuk and A. Kobushkin, Phys. Rev. D 79, 034001 (2009).

[22] M. Moteabbed et al., Phys. Rev. C 88, 025210 (2013).

[23] D. Yount and J. Pine, Phys. Rev. 128, 1842 (1962).

[24] A. Browman, F. Liu, and C. Schaerf, Phys. Rev. 139, B1079 (1965).

[25] R. L. Anderson et al., Phys. Rev. Lett. 17, 407 (1966).

[26] R. L. Anderson et al., Phys. Rev. 166, 1336 (1968).

[27] W. Bartel et al., Physics Letters B 25, 242 (1967).

[28] B. Bouquet et al., Physics Letters B 26, 178 (1968).

[29] J. Mar et al., Phys. Rev. Lett. 21, 482 (1968).

[30] A. Gramolin et al., Nucl.Phys.Proc.Suppl. 225, 216 (2012).

[31] R. Milner et al., Nucl. Instr. and Meth. A 741, 1 (2014).

[32] B. Mecking et al., Nucl. Instr. and Meth. A 503, 513 (2003).

[33] M. Niroula, Beyond the born approximation: a precise comparison of $e^{+} p$ and $e^{-} p$ elastic scattering in the CEBAF large acceptance spectrometer (CLAS), Ph.D. thesis, Old Dominion University (2010).

[34] M. Moteabbed, A Precise Measurement of the Two-Photon Exchange Effect, Ph.D. thesis, Florida International University (2009).

[35] M. Mestayer et al., Nucl. Instr. and Meth. A 449, 81 (2000).

[36] G. Adams et al., Nucl. Instr. and Meth. A 465, 414 (2001).

[37] E. Smith et al., Nucl. Instr. and Meth. A 432, 265 (1999).

[38] M. Amarian et al., Nucl. Instr. and Meth. A 460, 239 (2001).

[39] D. Rimal, Proton form factor puzzle and the CLAS two photon exchange experiment, Ph.D. thesis, Florida International University (2014). 
[40] A. Klimenko and S. Kuhn, CLAS Note 2003-005 (2003).

[41] R. Ent et al., Phys. Rev. C 64, 054610 (2001).

[42] P. G. Blunden, W. Melnitchouk, and J. A. Tjon, Phys. Rev. C 72, 034612 (2005).

[43] J. Arrington, W. Melnitchouk, and J. A. Tjon, Phys. Rev. C 76, 035205 (2007). 


\section{VITA}

Dasuni Kalhari Adikaram

Department of Physics

Old Dominion University

Norfolk, VA 23529

\section{Education}

Ph.D., Department of Physics, Old Dominion University, Norfolk, VA, May 2014 M.S., Department of Physics, Old Dominion University, Norfolk, VA, August 2009 B.S. in Physics, University of Colombo, Colombo, Sri Lanka, September 2006

\section{Academic Fellowships and Awards}

Jefferson Science Associates/Jefferson Lab Graduate Fellowship (2010 - 2011)

Award for the outstanding performance on the physics qualifying examination (2008)

Old Dominion University Graduate Fellowship (2007 - 2008)

\section{Conference Presentations}

American Physical Society April Meeting, Savannah, GA (2014)

Fall Meeting of the Division of Nuclear Physics of the American Physical Society, Newport News, VA (2013)

American Physical Society April Meeting, Denver, CO (2013)

5th Workshop of the American Physical Society Topical Group on Hadronic Physics, Denver, CO (2013)

American Physical Society April Meeting, Anaheim, CA (2011)

\section{Publications}

M. Moteabbed et al. Demonstration of a novel technique to measure two-photon exchange effects in elastic $e^{ \pm} p$ scattering. Phys. Rev. C88 (2010), p. 025210.

Typeset using $\mathrm{AT}_{\mathrm{E} X}$. 